\title{
Studies on the DEAD-box RNA-helicase Dbp5 and the $A B C$-protein Rli1 in translation termination and identification of a novel function of Dbp5 in ribosomal transport
}

\author{
Dissertation \\ for the award of the degree \\ "Doctor rerum naturalium" \\ of the Georg-August-Universität Göttingen \\ within the doctoral program "Molecular Biology of Cells" \\ of the Georg-August University School of Science (GAUSS) \\ submitted by \\ Bettina Neumann \\ (née Tieg) \\ from Zossen, Germany
}

Göttingen, February 2015 


\section{Members of the Thesis Committee}

Prof. Dr. Heike Krebber

Department of Molecular Genetics, Institute for Microbiology and Genetics

Prof. Dr. Gerhard Braus

Department of Molecular Microbiology and Genetics, Institute for Microbiology and Genetics

Prof. Dr. Marina Rodnina

Department of Physical Biochemistry, Max Planck Institute for Biophysical Chemistry

\section{Members of the Examination Board}

Referee: Prof. Dr. Heike Krebber

Department of Molecular Genetics, Institute for Microbiology and Genetics

$2^{\text {nd }}$ Referee: Prof. Dr. Gerhard Braus

Department of Molecular Microbiology and Genetics, Institute for Microbiology and Genetics

\section{Further members of the Examination Board}

PD Dr. Wilfried Kramer

Department of Molecular Genetics, Institute for Microbiology and Genetics

Prof. Dr. Ralf Ficner

Department of Molecular Structural Biology, Institute for Microbiology and Genetics

Prof. Dr. Ralph Kehlenbach

Department of Molecular Biology,

Faculty of Biochemistry

Prof. Dr. Stefanie Pöggeler

Department of Genetics of Eukaryotic Microorganisms, Institute for Microbiology and Genetics

Date of oral examination: $20^{\text {th }}$ April 2015 


\begin{abstract}
AFFIDAVIT
Hereby I declare that my doctoral thesis entitled "Studies on the DEAD-box RNAhelicase Dbp5 and the ABC-protein Rli1 in translation termination and identification of a novel function of Dbp5 in ribosomal transport" has been written independently with no other sources and aids than quoted.
\end{abstract}

Göttingen, February 2015

Bettina Neumann 


\section{TABLE OF CONTENTS}

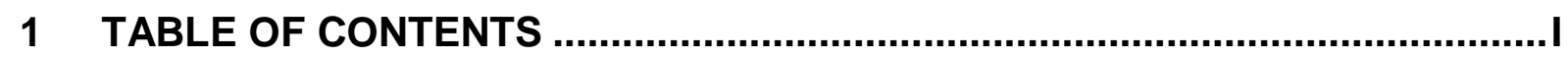

2 LIST OF FIGURES ................................................................................ IV

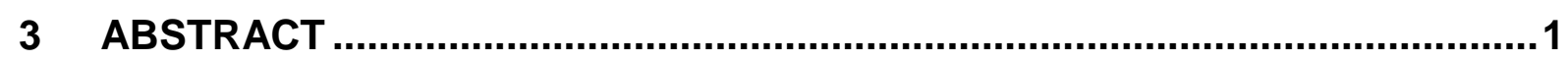

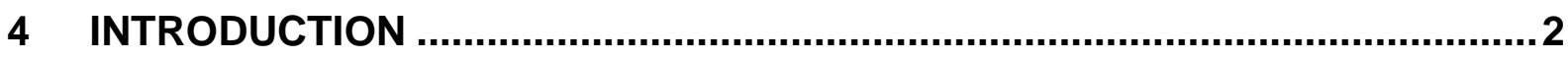

4.1 Biogenesis and nuclear export of eukaryotic pre-ribosomal particles................................2

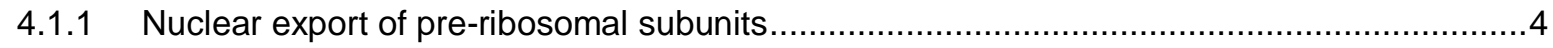

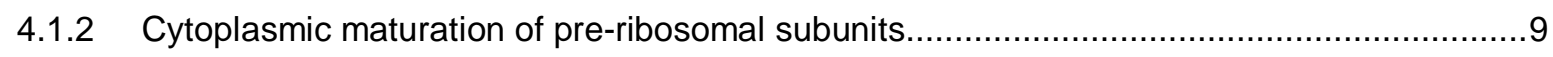

4.2 Eukaryotic translation termination and ribosome recycling .................................................

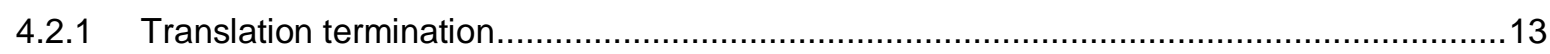

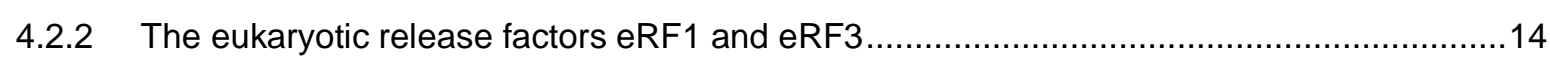

4.2.3 Model for the eRF1 and eRF3 mediated translation termination ...................................17

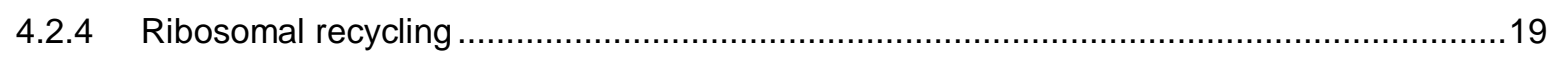

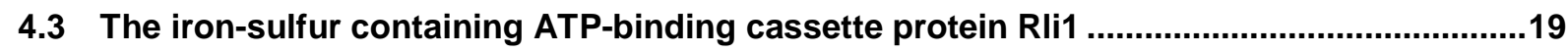

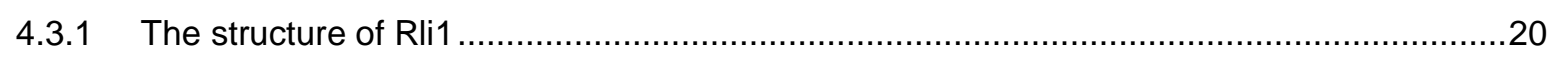

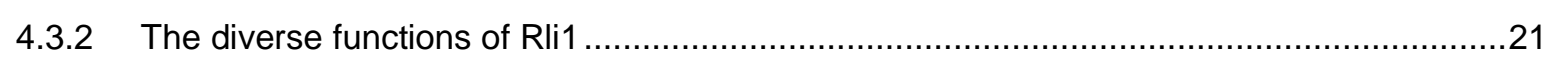

4.3.3 Rli1 in translation termination and ribosome recycling ....................................................22

4.4 The DEAD-Box RNA-helicase Dbp5/Rat8 and its co-factor Gle1 .......................................26

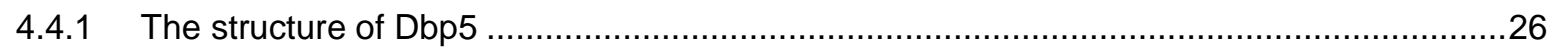

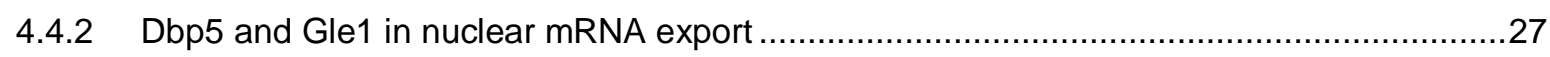

4.4.3 The regulation of the ATPase cycle of Dbp5 during mRNA export....................................29

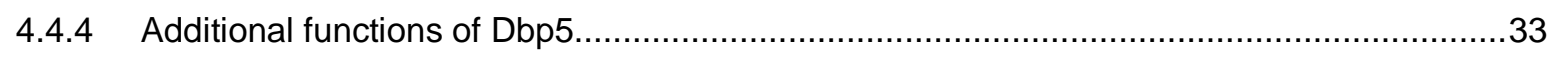

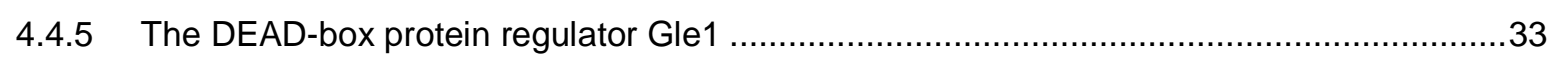

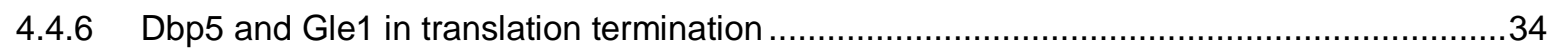

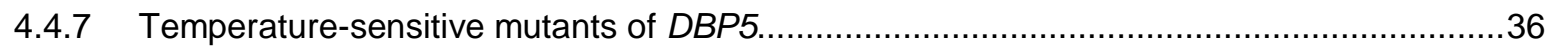

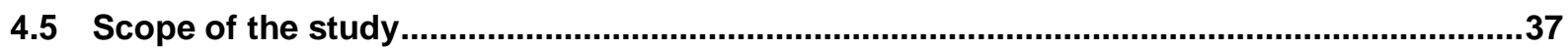

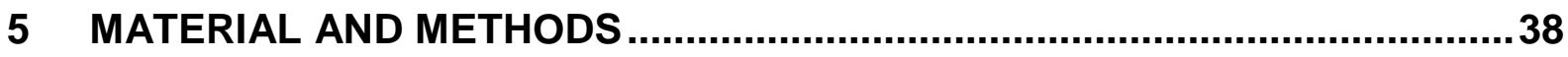

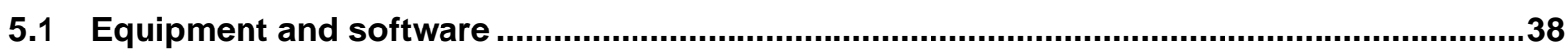

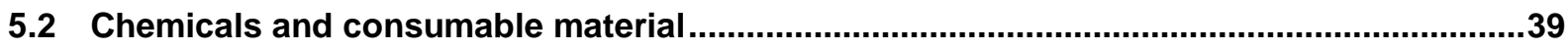

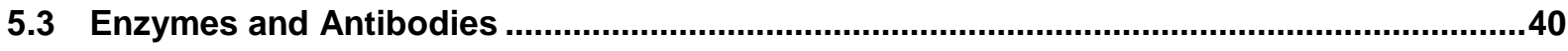

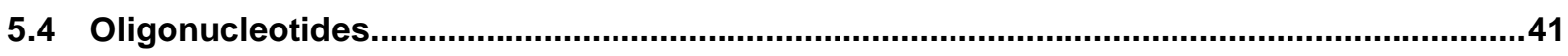

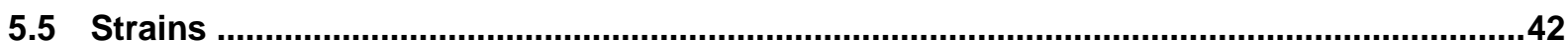




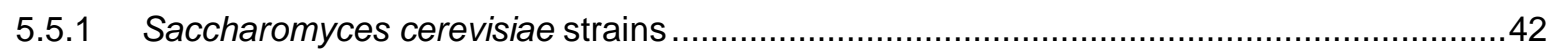

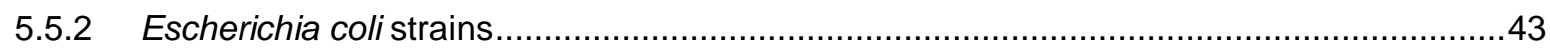

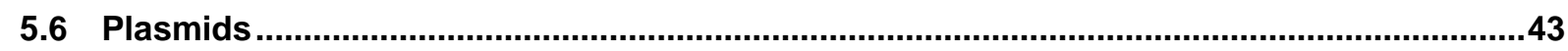

5.7 Cell cultivation .........................................................................................................44

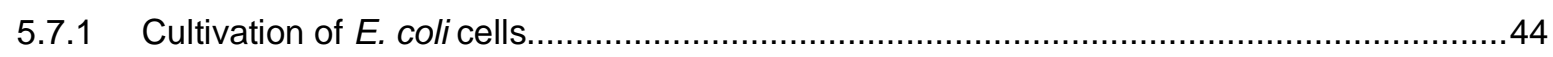

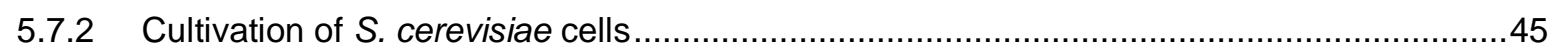

5.8 Generation 0 f yeast strains....................................................................................46

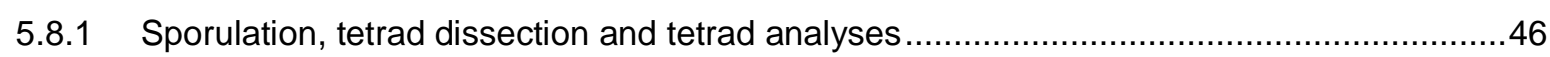

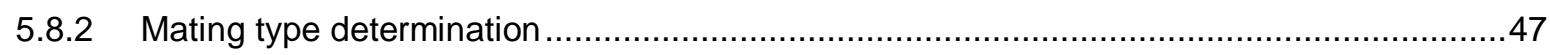

5.8.3 Generation of yeast strains HKY1356 and HKY1369-1372 .....................................48

5.9 Construction of recombinant DNA molecules ...............................................................48

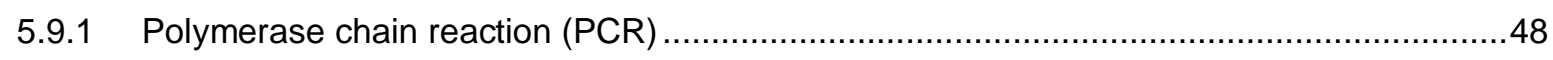

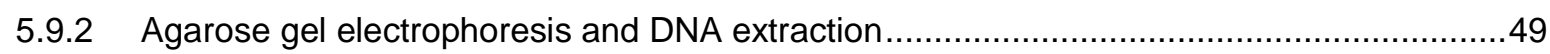

5.9.3 Restriction digest and dephosphorylation of 5'DNA-ends .....................................50

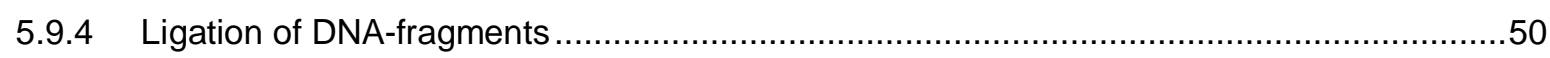

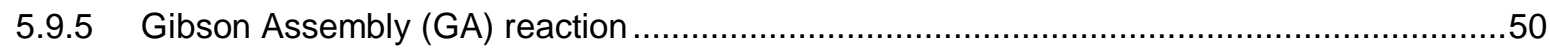

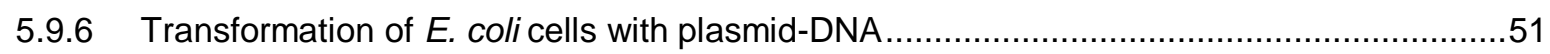

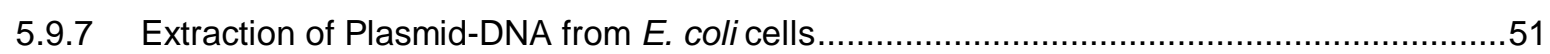

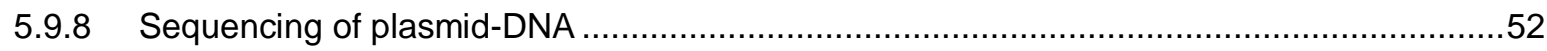

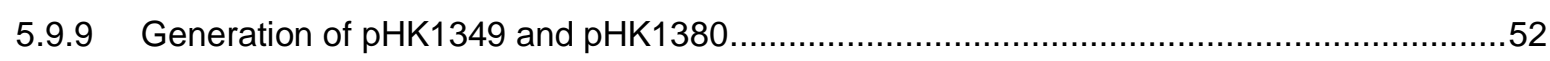

5.10 Molecular biological methods with yeast..................................................................52

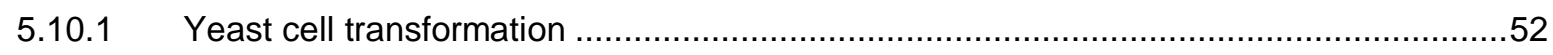

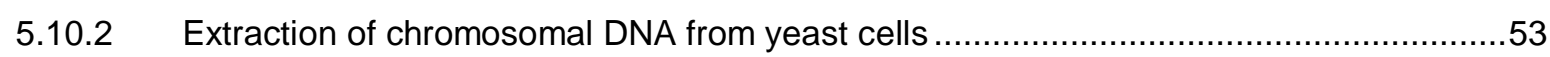

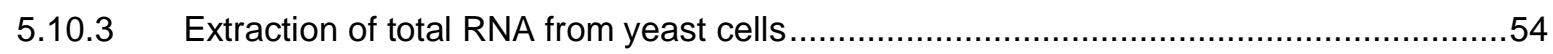

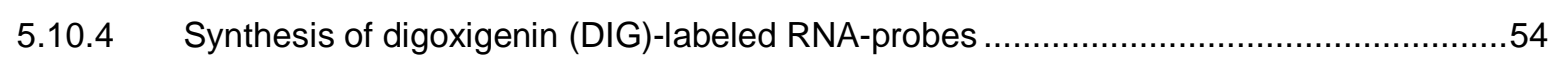

5.10.5 RNA-formaldehyde gels and Northern blotting ................................................55

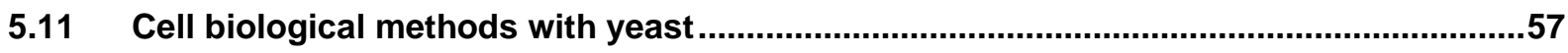

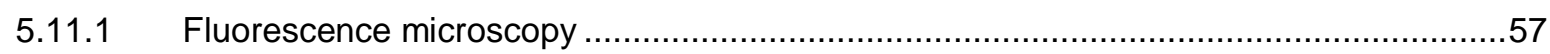

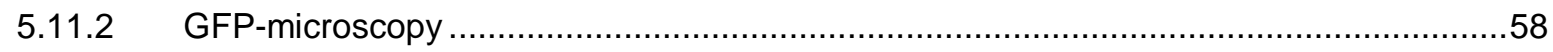

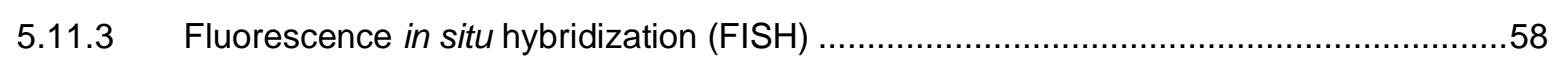

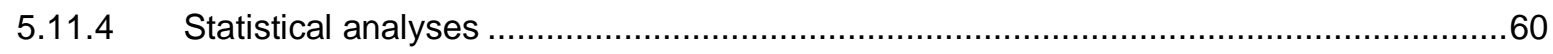

5.12 Protein biochemical methods................................................................................60

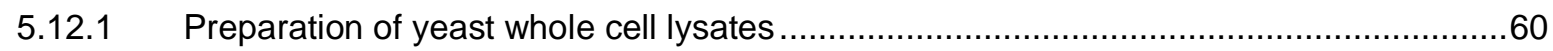

5.12.2 Co-immunoprecipitation studies for analyses of protein-protein interactions in vivo .......61

5.12.3 Expression of recombinant proteins and affinity purification for analyses of

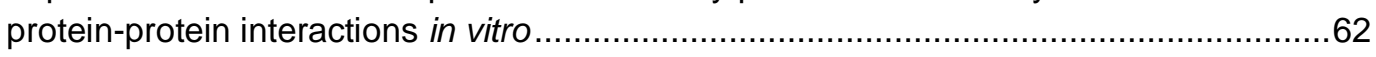

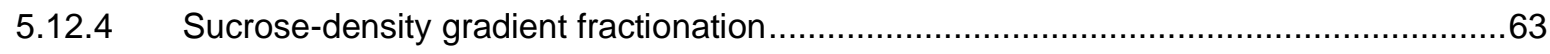

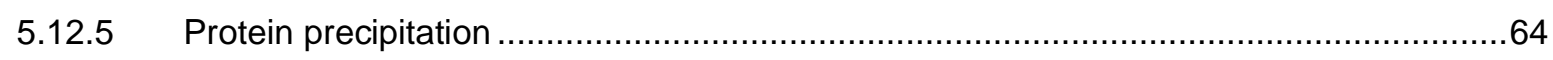




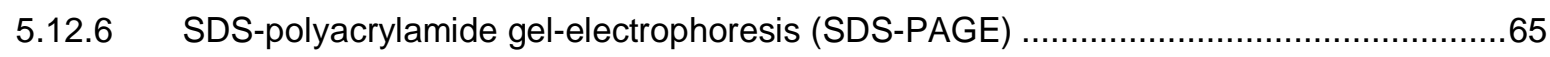

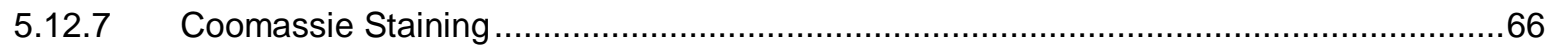

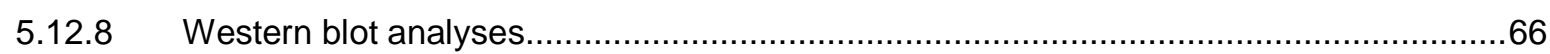

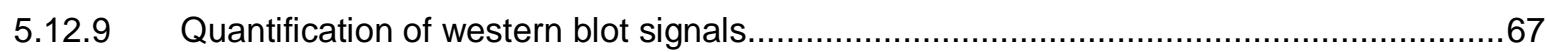

6 RESULTS .68

6.1 Analysis of the role of Dbp5 in the nuclear export of ribosomal particles .........................68

6.1.1 Mutants of $D B P 5$ are defective in the nuclear export of pre-40S and pre-60S particles.....68

6.1.2 The biogenesis of ribosomal subunits is not obviously affected in the $d b p 5$ mutants..........73

6.1.3 Dbp5 genetically and physically interacts with established ribosomal transport factors ......76

6.1.4 Dbp5 rather acts at the cytoplasmic side of the NPC than on ribosomal particles

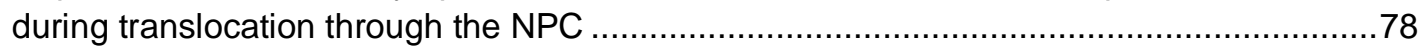

6.1.5 Dbp5 does not displace Mex67 from exported pre-ribosomal subunits...............................82

6.1.6 The ATPase cycle of Dbp5 is dispensable for ribosomal transport ....................................85

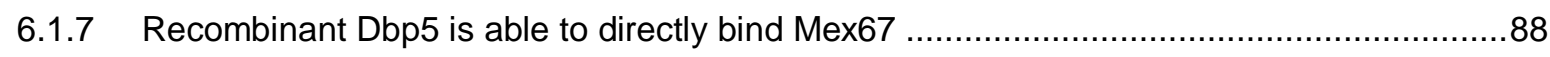

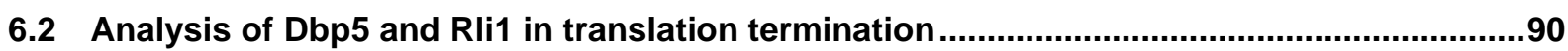

6.2.1 Rli1 interacts with Dbp5 and its co-factor Gle1 during translation termination....................90

6.2.2 The ribosomal association of Dbp5 is dependent on functional eRF1 ...............................94

6.2.3 The association of Rli1 with ribosomal proteins is not decreased without functional eRF1.97

7 DISCUSSION .99

7.1 The DEAD-box RNA-helicase Dbp5 is ATPase-independently required for the nuclear export of both pre-ribosomal subunits ....................................................................99

7.1.1 Dbp5 is directly required for the nuclear export of pre-60S and pre-40S particles .............99

7.1.2 The ATPase-dependent RNP remodeling of Dbp5 is dispensable

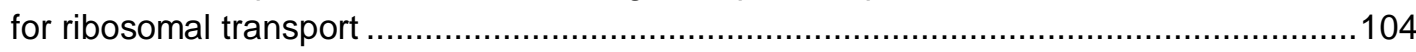

7.1.3 How might Dbp5 contribute to the nuclear export of ribosomal particles? .........................109

7.2 Dbp5 and Rli1 interact during translation termination

7.2.1 Dbp5, its co-factor Gle1 and Rli1 interact with each other during translation termination .113

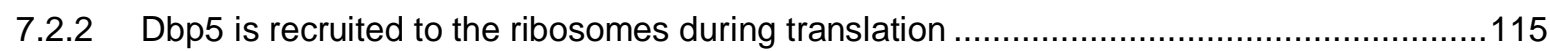

7.2.3 Is Rli1 eRF1-independetly recruited to the ribosomes? .................................................117

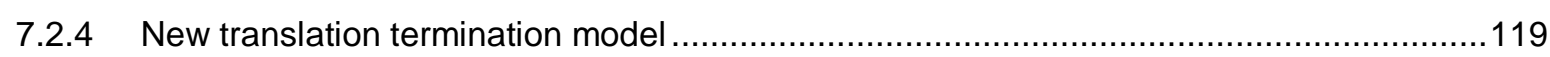

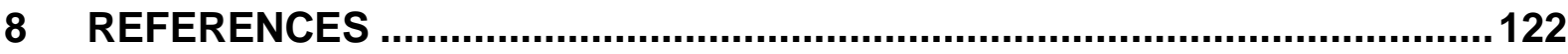

9 ACKNOWLEDGEMENTS - DANKSAGUNG ............................................ 133

10 CURRICULUM VITAE ............................................................................ 134 


\section{LIST OF FIGURES}

Figure 1: Scheme of the ribosomal biogenesis and processing of the ribosomal RNAs.......................

Figure 2: Scheme of the nuclear export of pre-ribosomal subunits. ................................................... 5

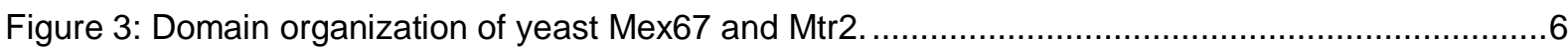

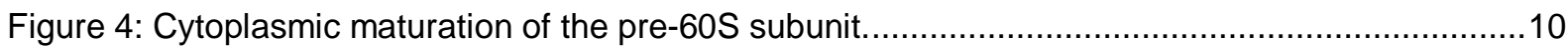

Figure 5: Cytoplasmic maturation and quality control of the pre-40S subunit..................................12

Figure 6: Structure and domains of the eukaryotic release factor eRF1

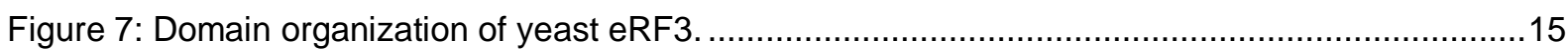

Figure 8: Model of the eRF1 and eRF3 mediated translation termination. .......................................18

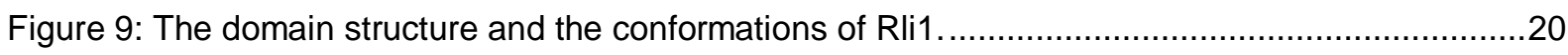

Figure 10: Model for translation termination and ribosome recycling mediated by Rli1......................25

Figure 11: The domain structure of Dbp5 and its binding regions for interaction partners. .................27

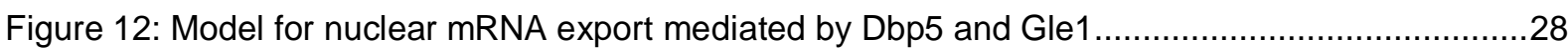

Figure 13: Scheme of the ATPase cycle of Dbp5 during nuclear mRNA export...............................31

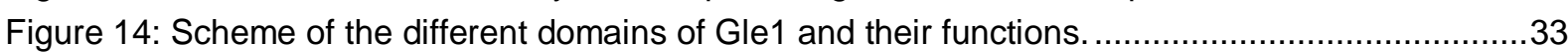

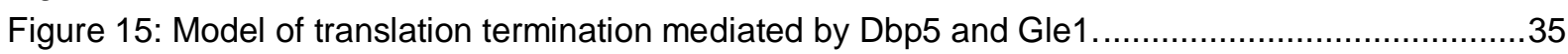

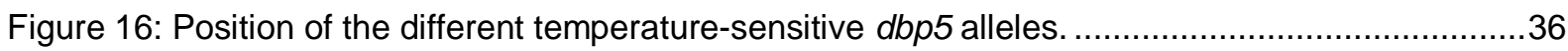

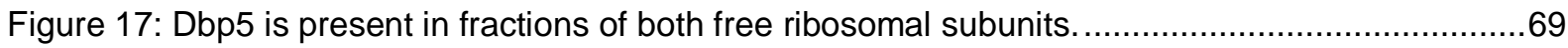

Figure 18: Both pre-ribosomal subunits accumulate in the nuclei of different $d b p 5$ mutants...............70

Figure 19: The ribosomal export defects do not correlate with the mRNA export defects

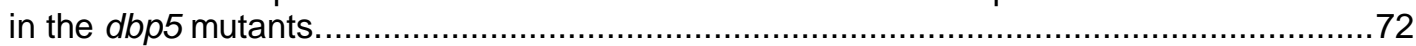

Figure 20: The steady state level of the different rRNA species in dbp5 mutants is comparable

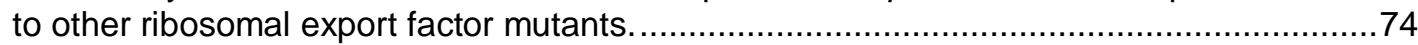

Figure 21: The total amount of ribosomal subunits is unchanged in the dbp5 mutants.......................75

Figure 22: Dbp5 genetically interacts with the ribosomal transport factors Nmd3 and Mtr2 ................76

Figure 23: Dbp5 physically interacts with the ribosomal export factors Xpo1 and Nmd3. ...................77

Figure 24: Dbp5 is not transferred together with the ribosomal particles into the cytoplasm...............79

Figure 25: Dbp5 does not accumulate with Nmd3 on immature cytoplasmic ribosomal particles in $\operatorname{drg} 1-18$ cells.

Figure 26: Slight nuclear accumulation of ribosomal particles is visible in rat7-1 cells........................81

Figure 27: Mex67 is associated with polysomes and not displaced from ribosomal particles by Dbp5.

Figure 28: The ribosomal association of Mex67 is unchanged in the $d b p 5$ mutants. .........................85

Figure 29: Missing ATPase stimulation of Dbp5 does not cause severe ribosomal export defects......86

Figure 30: ATPase-deficient $d b p 5$ mutants show no obvious ribosomal export defects.......................87

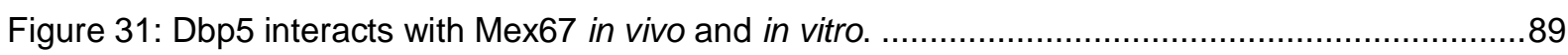

Figure 32: Rli1 interacts RNA-independently with Dbp5 and Gle1 in vivo.......................................

Figure 33: The interaction of Rli1 with Dbp5 and Gle1 is decreased without functional eRF1. ...........92

Figure 34: The interaction between Rli1 and Dbp5 is slightly decreased without proceeding of

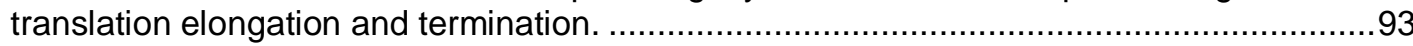

Figure 35: The binding of Dbp5 to ribosomal proteins is decreased without functional eRF1............95

Figure 36: The eRF1 mutant sup45-2 shows a polysome run-off and an increased 80S peak. ..........96

Figure 37: Dbp5 is less associated with the ribosomal fractions of sup45-2 cells. .............................96

Figure 38: Rli1 binds stronger to Rps3, but its binding to Rpl35 is unchanged in sup45-2 ................98

Figure 39: Model for the differences in Dbp5-mediated nuclear export of mRNAs and

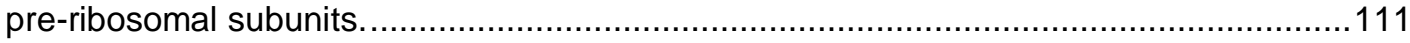

Figure 40: Suggested model for the chronology of translation termination.....................................120 


\section{ABSTRACT}

The DEAD-box RNA-helicase Dbp5/Rat8 is well-known for its function in nuclear

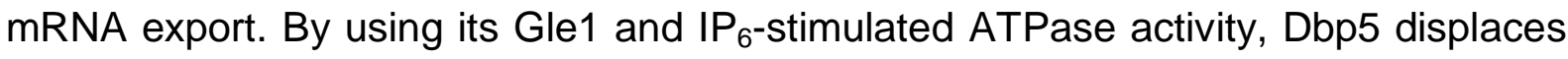
export factors such as Mex67 from the mRNAs at the cytoplasmic sides of the nuclear pore complexes. This Dbp5-mediated remodeling of the emerging mRNPs is anticipated to enable their directional transport from the nucleus into the cytoplasm. The present study shows that Dbp5 is also required for the nuclear export of other large ribonucleoprotein complexes, the pre-ribosomal subunits. GFP-microscopy and fluorescence in situ hybridization experiments in Saccharomyces cerevisiae reveal that both, pre-40S and pre-60S particles accumulate in the nuclei of different $d b p 5$ mutants. Like in mRNA export, Dbp5 acts also in ribosomal transport at the cytoplasmic sides of the nuclear pore complexes, where it shortly contacts the emerging ribosomal particles. However, Dbp5 utilizes for the export of pre-ribosomal subunits a mechanism that is distinct from its role in mRNA transport. Dbp5 does not displace the export receptor Mex67 from the pre-ribosomal subunits and does not require its Gle1-stimulated ATPase cycle for ribosomal transport. Thus, the ATPasedependent remodeling activity of Dbp5 in general is dispensable for the nuclear export of pre-ribosomal subunits. These findings uncover differences in the transport mechanisms of Dbp5 for different large ribonucleoprotein particles.

In addition to the roles in nuclear export events, Dbp5 and its co-factor Gle1 are also involved in efficient translation termination in the cytoplasm. Translation termination takes place upon arrival of the translating ribosomes at the stop codon and requires the canonical release factors eRF1 and eRF3 to mediate stop codon recognition and peptide release. Furthermore, the ATP-binding cassette protein Rli1 was described as an additional termination factor. However, the chronological order of all factors acting during translation termination is still unknown. Co-immunoprecipitation and sucrose-density gradient studies reveal a novel interaction between Dbp5 and Rli1 during translation termination and an early entry of Dbp5 into the termination complex that might depend on eRF1. Dbp5 is recruited to the ribosomes after Rli1, which already associates with ribosomes prior to translation termination. Thus, a ribosomemediated contact between Rli1 and Dbp5 is possible either before or during the eRF1-mediated stop codon recognition. These findings present important insights into the temporal coordination of all termination factors during translation termination. 


\section{INTRODUCTION}

\subsection{Biogenesis and nuclear export of eukaryotic pre-ribosomal particles}

Eukaryotic 80S ribosomes consist of the small (40S) and the large (60S) subunit, which are build up by ribosomal proteins and ribosomal RNAs (rRNAs). In the budding yeast Saccharomyces cerevisiae, the 40S subunit comprises the 18S rRNA and 33 small ribosomal proteins (Rps), whereas the 60S subunit contains the 25S, $5 \mathrm{~S}$ and 5.8S rRNAs and 46 large ribosomal proteins (Rpl) (Woolford and Baserga, 2013). Saccharomyces cerevisiae is a common used model organism to analyze ribosomal biogenesis and nuclear transport mechanisms. Thus, the present study is mainly focusing on this organism. Nevertheless, most of the proteins and their functions described in the following are conserved in higher eukaryotes.

The biogenesis of the ribosomal subunits begins in the nucleolus with the transcription of the ribosomal DNA (rDNA) repeats and the generation of a common $35 \mathrm{~S}$ precursor transcript by the RNA-polymerase I (Figure 1A) (Gerhardy et al., 2014). This transcript contains the 18S, $5.8 \mathrm{~S}$ and 25S rRNA sequences, which are separated by external (ETS) and internal transcribed spacer (ITS) regions (Figure 1B). The 5S rRNA is separately transcribed by the RNA-polymerase III and associates later with the large pre-ribosomal subunit, but the exact time point for its incorporation is not clear yet (Figure 1) (Ciganda and Williams, 2011). Already during transcription, multiple assembly factors and predominantly small ribosomal proteins associate with the $35 \mathrm{~S}$ pre-rRNA to form the $90 \mathrm{~S}$ particle or the small subunit processome (Figure 1A) (Thomson et al., 2013). Furthermore, small nucleolar ribonucleoprotein (snoRNP) particles act co-transcriptionally to covalently modify the pre-rRNAs, whereat 2'-O-methylation is mediated by C/D box containing snoRNPs and pseudiuridinylation by H/ACA box containing snoRNPs (Thomson et al., 2013). 

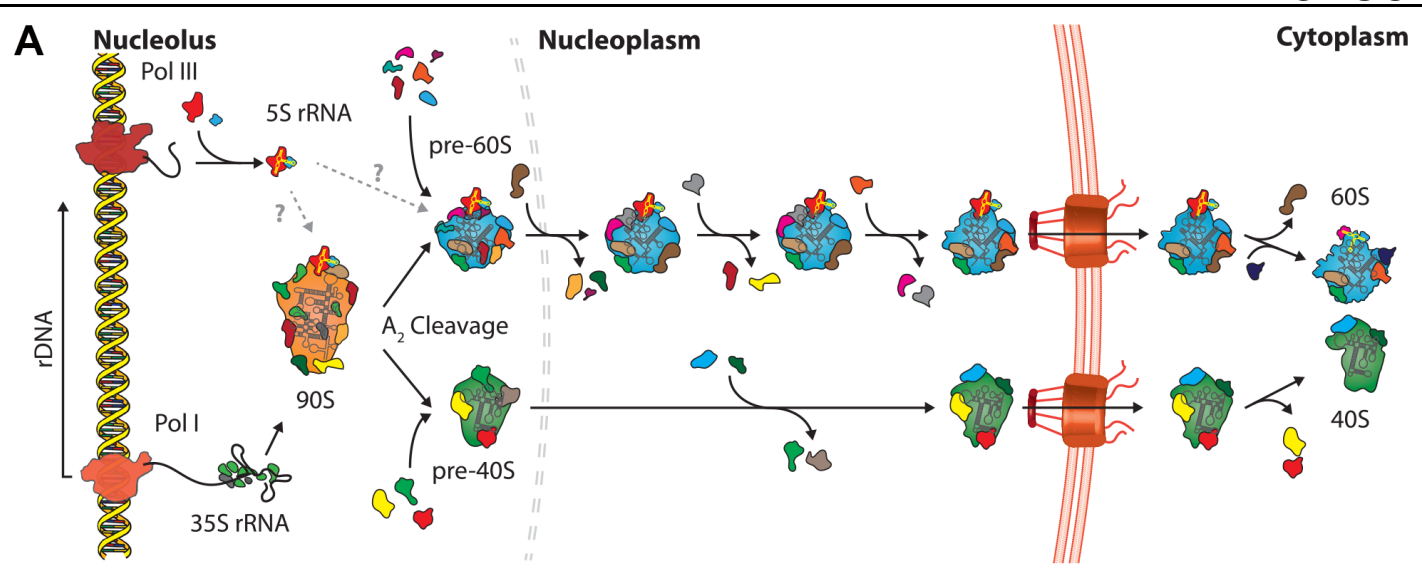

B

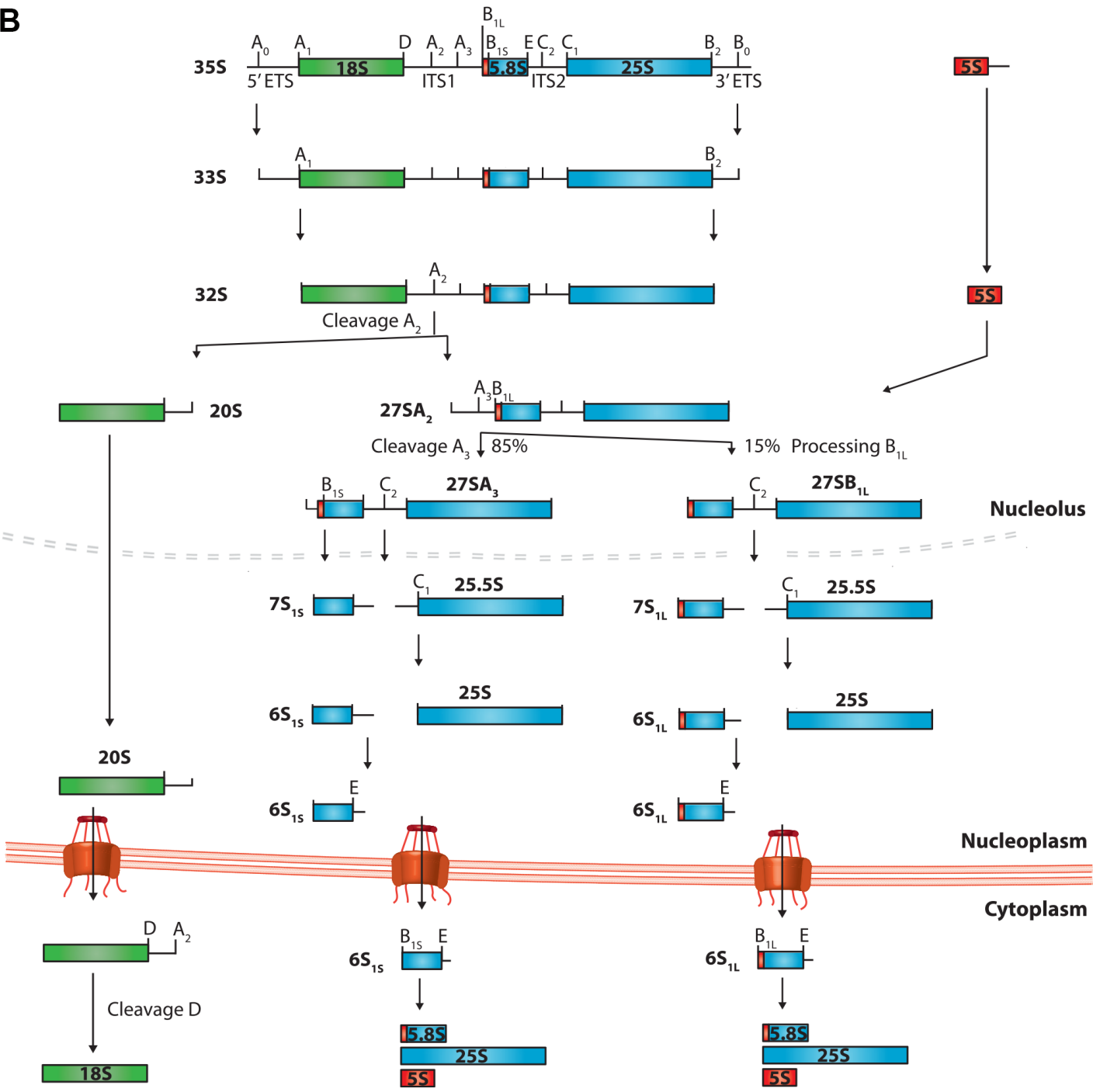

Figure 1: Scheme of the ribosomal biogenesis and processing of the ribosomal RNAs.

(A) The pathway to generate ribosomal subunits is schematically shown. In the nucleolus, a common 35S rRNA transcript is synthesized from the rDNA by the RNA-polymerase I (Pol I), while the 5S rRNA is separately transcribed by the RNA-polymerase III (Pol III). Ribosomal proteins and various biogenesis factors co-transcriptionally associate to form the $90 \mathrm{~S}$ particle. Cleavage at site $\mathrm{A}_{2}$ leads to the generation of the pre-60S (in blue) and the pre-40S (in green) subunits and the separation of their biogenesis pathways. Additional biogenesis steps of the pre-60S particle occur in the nucleoplasm, whereas the pre-40S is instantly transported into the cytoplasm, where further cytoplasmic maturation of both subunits takes place. (B) A scheme of the rRNA processing is shown. The $35 \mathrm{~S}$ pre-rRNA contains the $18 \mathrm{~S}, 5.8 \mathrm{~S}$ and $25 \mathrm{~S}$ rRNA sequences and external (ETS) and internal transcribed spacer (ITS). The different cleavage sites $A_{0}-E$ of the $35 S$ pre-rRNA are indicated. The ETS and ITS regions are removed by a series of endo- and exonucleolytic cleavage events leading to the different intermediates and finally to the mature rRNAs, as schematically shown. The 5S rRNA is separately processed. Modified from Gerhardy et al. (2014). 
Processing of the $35 \mathrm{~S}$ pre-rRNA is mediated by a series of endo- and exonucleolytic cleavage events, which remove the ETS and ITS sequences and produce the mature rRNAs (Figure 1B) (Gerhardy et al., 2014). Cleavage at site $A_{2}$ leads to the generation of the $20 \mathrm{~S}$ and the $27 \mathrm{SA}_{2}$ pre-rRNAs and consequently to the splitting of the $90 \mathrm{~S}$ particle into the pre-40S and pre-60S subunits (Figure 1). Their biogenesis pathways are now separated and the $27 \mathrm{SA}_{2}$ pre-rRNA undergoes further cleavage steps in two alternative pathways to finally produce the mature 5.8S and 25S rRNAs (Figure 1B) (Woolford and Baserga, 2013).

If mutants are defective or delayed in the early processing events, the premature splitting of the $35 \mathrm{~S}$ pre-rRNA at site $A_{3}$ can occur prior to cleavage at sites $A_{0}, A_{1}$ and $\mathrm{A}_{2}$ (Venema and Tollervey, 1999). Such processing defects cause the production of an aberrant 23S rRNA intermediate, which is no substrate for the processing machinery and leads to reduced $20 \mathrm{~S}$ and $18 \mathrm{~S}$ rRNA levels. Contrary, the $27 \mathrm{SA}_{3}$ prerRNA as second product of the premature $A_{3}$ cleavage can undergo normal processing producing mature rRNAs of the large ribosomal subunit.

Generally during maturation, the composition of the ribosomal particles, especially of the pre-60S, constantly changes and a great number of biogenesis and export factors are needed for the generation of translation-competent ribosomal subunits. Especially, energy-consuming enzymes like ATP-dependent RNA-helicases, kinases, AAA-ATPases, ABC-proteins and GTPases are necessary to direct the maturation process (Kressler et al., 2010). The pre-40S particle undergoes most of its maturation steps in the nucleolus, where it contains still the immature 205 pre-rRNA. Upon nucleolar exit, this complex is quickly transported through the nucleoplasm and the nuclear pore complexes (NPCs) into the cytoplasm, where its biogenesis and the processing of the $20 \mathrm{~S}$ into the mature $18 \mathrm{~S}$ rRNA is completed. In contrast, the pre605 subunit traverses through multiple biogenesis steps in the nucleoplasm, before the nuclear export and final cytoplasmic maturation takes place.

\subsubsection{Nuclear export of pre-ribosomal subunits}

The compartmentation of the eukaryotic cell into nucleus and cytoplasm requires active transport mechanisms to transfer particles between both compartments. Thus, NPCs are embedded in the nuclear envelope and serve as gates for the selected transport of macromolecules larger than $\sim 40 \mathrm{kDa}$ (Wente and Rout, 2010). These eightfold symmetric supramolecular structures are built up from repeated nucleoporins and consist of the core scaffold, the central channel for the passage, 
the nuclear basket and the cytoplasmic filaments. In S. cerevisiae, the cytoplasmic filaments are comprised of the nucleoporins Nup159/Rat7 and Nup42. The channels are build up by phenylalanine-glycine-repeats (FG-repeats) containing nucleoporins, which form a hydrophobic meshwork that limits diffusion of large and charged particles. For selected transport in general, shuttling transport factors (mostly karyopherins) bind to the transport signal containing cargo and directly interact with the hydrophobic FG-repeats in the channels allowing the translocation of their cargos (Strambio-De-Castillia et al., 2010). For ribosomal export, various export factors need to bind to different sites of the pre-ribosomal subunits to facilitate the rapid transport of these huge and charged particles through the NPCs into the cytoplasm (Figure 2) (Gerhardy et al., 2014).

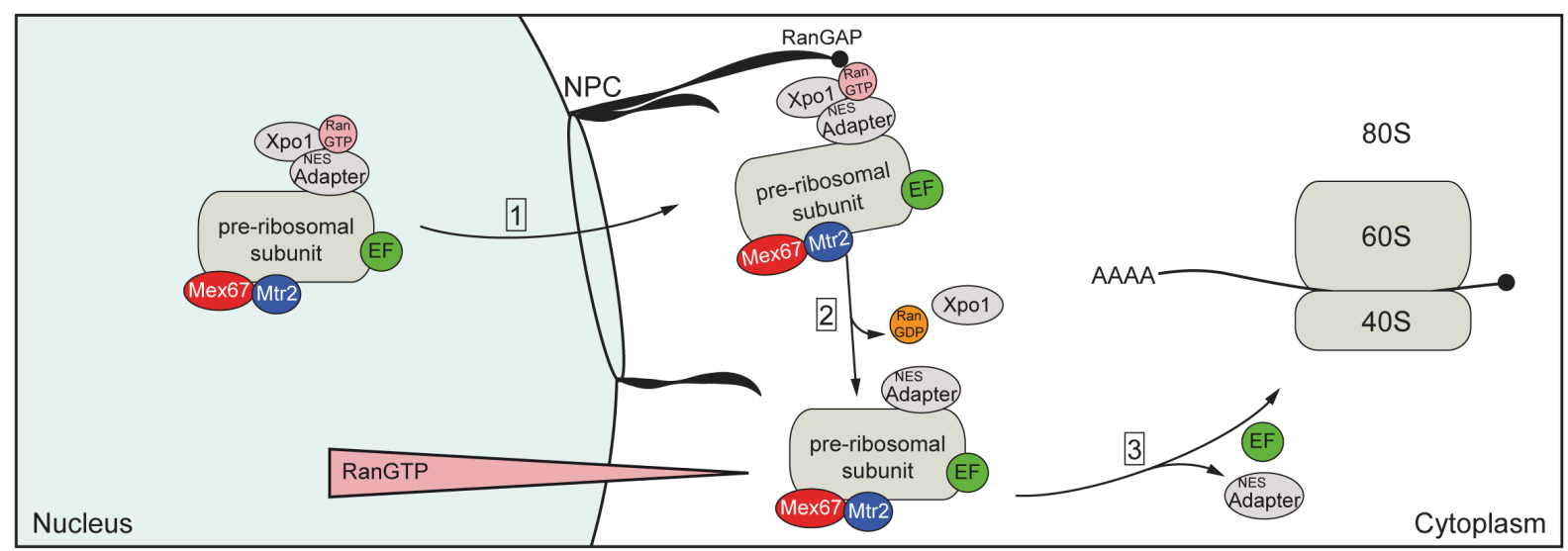

Figure 2: Scheme of the nuclear export of pre-ribosomal subunits.

Both pre-ribosomal subunits utilize the karyopherin Xpo1/Crm1 and the Ran GTPase cycle for their transport. In the nucleus, Xpo1 binds with RanGTP to the NES-containing adapter protein (Nmd3 for pre-60S, unknown for pre-40S) and facilitates translocation of the pre-ribosomal subunit through the nuclear pore complex (NPC) (1). Mex67-Mtr2 and additional subunit-specific export factors (EF in green) need to cover the huge particles and interact with the FG-nucleoporins of the NPCs. In the cytoplasm, RanGAP stimulates GTP-hydrolysis of Ran, which causes the dissociation of Xpo1 and RanGDP and thereby ensures the irreversibility of the transport (2). During cytoplasmic maturation, the export factors are released leading to ribosomal subunits competent for translation (3).

Nuclear export of pre-40S and pre-60S particles involves the karyopherin Xpo1 (Crm1/exportin 1) and the Ran GTPase cycle, which are both conserved in higher eukaryotes (Gadal et al., 2001; Ho et al., 2000; Hurt et al., 1999; Moy and Silver, 1999; Thomas and Kutay, 2003; Trotta et al., 2003). The spatial regulated GTPase cycle of the small GTPase Ran (yeast Gsp1) ensures directionality of the transport process by generation of a RanGTP gradient across the nuclear envelope (Grunwald et al., 2011). This gradient is formed due to the localization of the Ran guanine nucleotide exchange factor (RanGEF, yeast Prp20) in the nucleoplasm and the Ran GTPase activating protein (RanGAP, yeast Rna1) in the cytoplasm (Wente and Rout, 
2010). Thus, GTP-bound Ran is predominantly localized in the nucleus, where it facilitates the association of Xpo1 with the pre-ribosomal particles (Figure 2). The subsequent translocation through the NPC is mediated by the interaction of Xpo1 with the FG-repeats. Upon arrival in the cytoplasm, RanGAP stimulates the GTPhydrolysis of Ran, which leads to the dissociation of RanGDP and Xpo1 leaving the ribosomal particles in the cytoplasm.

However, Xpo1 does not bind directly to the pre-ribosomal subunits, but rather needs the nuclear export signal (NES)-containing adaptor protein Nmd3 for its association with pre-60S particles (Gadal et al., 2001; Ho et al., 2000; Thomas and Kutay, 2003; Trotta et al., 2003). In contrast to the large pre-ribosomal subunits, the NEScontaining pre-40S adapter protein for Xpo1 is still unknown. Even though the pre$40 \mathrm{~S}$ transport is influenced by the biogenesis factors Ltv1, Dim2 and Rio2, which contain potential NESs and are suggested as candidates that contribute to the Xpo1 recruitment, these NES sequences are not essential for viability and pre-40S export (Merwin et al., 2014; Seiser et al., 2006; Vanrobays et al., 2008; Zemp et al., 2009). Possibly, not just one essential adapter might recruit Xpo1 to the pre-40S subunit, but rather several factors that compensate for each other.

Furthermore, the transport of both pre-ribosomal subunits requires the export receptor heterodimer Mex67-Mtr2 (human TAP-p15), which does not depend on the Ran GTPase cycle (Figure 2) (Faza et al., 2012; Yao et al., 2007). Mex67-Mtr2 is also needed for the nuclear mRNA export (Santos-Rosa et al., 1998; Segref et al., 1997). However, alleles such as mtr2-33 exist that are only impaired in ribosomal, but not in mRNA export (Bassler et al., 2001). Generally, both proteins contain nuclear transport factor (NTF2)-like domains, which enable their heterodimerization and directly interact with the FG-repeats of the NPCs (Figure 3) (Gerhardy et al., 2014).

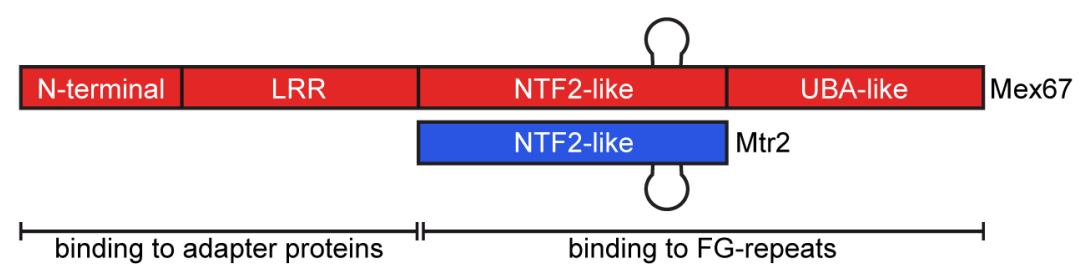

Figure 3: Domain organization of yeast Mex67 and Mtr2.

Mex67 (in red) contains the $\mathrm{N}$-terminal domain and the leucine-rich repeat (LRR), which interact with adapter proteins during mRNA export. These domains are followed by the nuclear transport factor (NTF2)-like and the ubiquitin-associated (UBA)-like domains, which bind the FG-repeats. Mtr2 (in blue) consists only of a NTF2-like domain, which interacts with FG-repeats and Mex67. Both proteins contain yeast specific loops important for the interaction with the rRNAs of preribosomal subunits. Modified from Faza et al. (2012). 
In yeast, the NTF2-like domains of Mex67 and Mtr2 contain specific loops, which are critical for the interaction with the pre-60S and pre-40S particles and their nuclear export (Figure 3) (Faza et al., 2012; Yao et al., 2007). In particular, the positively charged surface provided by these loops can directly interact with the negatively charged backbone of the double-stranded 5S rRNA, which is exposed from the pre$60 S$ subunit (Yao et al., 2007). This interaction site is opposite of the hydrophobic FG-repeat binding site of the heterodimer enabling its simultaneous binding to the pre-60S particle and the FG-nucleoporins. This ability suggests Mex67-Mtr2 as an export receptor that supports the translocation of the pre-60S subunit (Yao et al., 2007). Likewise, these loops of Mex67-Mtr2 are necessary for the transport of the pre-40S subunit, but the exact interaction site between Mex67-Mtr2 and the pre-40S particle has not been identified yet (Faza et al., 2012). Large-scale crosslinking studies showed a crosslink between Mex67 and the 20S rRNA suggesting a similar RNA-based contact for the small pre-ribosomal subunit (Gerhardy et al., 2014; Tuck and Tollervey, 2013). Recent structural analyses revealed that even the N-terminal and the LRR domain of Mex67 contribute in addition to the NTF2-like domains to a large positively charged surface of one side of the heterodimer, which might represent the general RNA binding site (Aibara et al., 2015).

Several other shuttling export factors for the pre-60S subunits are known like Npl3 (Hackmann et al., 2011), the non-essential Bud20 (Altvater et al., 2012; Bassler et al., 2012), Arx1 (Bradatsch et al., 2007; Hung et al., 2008) and Ecm1 (Bassler et al., 2001; Yao et al., 2010). These factors support the passage of the pre-60S subunits through the hydrophobic interior of the NPCs by shielding the particles at different sites and by their simultaneous direct interaction with the FG-repeats. Recently, the GTPase Nug2/Nog2 and the ATPase Rea1 were anticipated to control the time point of the pre-60S export, so that completion of the nuclear maturation prior to transport is ensured (Matsuo et al., 2014). The authors revealed that Nug2 and Nmd3 share their ribosomal binding sites indicating that their association with the pre-60S subunit must be mutually exclusive. Only when Nug2 is released by Rea1 from the nuclear pre-60S subunit, Nmd3 can associate and recruit Xpo1 and RanGTP for nuclear export.

Less is known about the transport of the small pre-ribosomal subunit. Beside the established transport factors Mex67-Mtr2, Xpo1, potential adapters and the Ran GTPase cycle, some other factors are implicated to support its nuclear export. However, the exact transport mechanisms are not understood. Screening of 
temperature-sensitive yeast libraries with pre-40S export assays particularly identified nucleoporins and Ran regulators to be involved in this process (Moy and Silver, 1999, 2002). For example, cells lacking the nuclear Ran-binding protein Yrb2 are delayed in pre-40S export (Moy and Silver, 2002). Furthermore, the HEAT-repeat containing protein Rrp12 directly interacts with the FG-repeats and RanGTP and was initially anticipated to support transport of both pre-ribosomal subunits, since depletion of Rrp12 leads to their nuclear accumulation (Oeffinger et al., 2004). However, a recent study suggested that Rrp12 is especially needed for the nuclear export of the pre-40S subunit (Moriggi et al., 2014). The authors showed that Xpo1 is recruited early and depending on Rrp12 to the $90 S$ particle, on which they are necessary for the proper processing of the $35 \mathrm{~S}$ pre-rRNA and subsequent pre-40S export. Moreover, the biogenesis factors Enp1, Bud23, Tsr1, Hrr25 and Rio2 might also be required for export, as their mutation or depletion, respectively, lead to the nuclear accumulation of pre-40S reporters (Schafer et al., 2006; Schafer et al., 2003; White et al., 2008). However, whether these factors directly mediate the nuclear export of the small pre-ribosomal subunit or are rather involved in its maturation needs to be elucidated.

Since the early 2000s, potential export factors were identified in S. cerevisiae by monitoring GFP-tagged ribosomal proteins as reporters for the nuclear mislocalization of ribosomal subunits in mutant strains (Gadal et al., 2001; Hurt et al., 1999; Milkereit et al., 2003; Stage-Zimmermann et al., 2000). As free ribosomal proteins that are not incorporated in the ribosomal subunits will be rapidly degraded, the nuclear signal of the GFP-tagged ribosomal proteins indeed reflect the complete ribosomal particles (Ho et al., 2000). In wild type cells, most of these reporters are integrated in mature ribosomal subunits leading to a predominant staining of the cytoplasm. In contrast, export mutants show a predominant nuclear signal, as the GFP-tagged ribosomal proteins representing the pre-ribosomal particles accumulate in the nucleoplasm of these cells. However, also mutants of factors involved in the ribosomal maturation can lead to delayed or disturbed transport and might show this phenotype. This is especially true for the small subunit, of which biogenesis and transport are tightly coupled (Tschochner and Hurt, 2003). Thus, a nuclear accumulation of the reporter in an export assay does not automatically proof that the corresponding factor is directly involved in ribosomal transport. Established reporters for the export of the large ribosomal subunit are Rpl11b-GFP (Stage-Zimmermann et al., 2000) and Rpl25-GFP (Gadal et al., 2001; Hurt et al., 1999), while Rps2-GFP 
serves as reporter for the small ribosomal subunit (Milkereit et al., 2003).

In another established assay, screens for pre-40S export defects are performed by in situ hybridizations with labeled probes against the 5'ITS1 rRNA (Gleizes et al., 2001; Moy and Silver, 1999, 2002). This sequence represents the 3'end of the 20 S prerRNA that is excised and degraded in the cytoplasm of wild type cells (Figure 1). In this experiment, wild type cells show only a staining of the nucleolus. In contrast, in pre-40S export mutants, in which this cytoplasmic processing cannot occur, a staining of the complete nucleoplasm is visible (Moy and Silver, 2002). To distinguish also between assembly and export defects, xrn1 strains lacking the 5'ITS1 degrading enzyme were used (Moy and Silver, 1999). In such assay, wild type cells show a cytoplasmic staining, whereas mutants with a defect in the ribosomal assembly accumulate the signal in the nucleolus and export defect mutants in the nucleoplasm (Moy and Silver, 1999).

\subsubsection{Cytoplasmic maturation of pre-ribosomal subunits}

In the cytoplasm, the bound shuttling biogenesis and export factors are dissociated from the pre-ribosomal subunits to complete their maturation into translationcompetent ribosomal subunits. Furthermore, the released factors have to be returned to the nucleus for the next round of maturation and export. The cytoplasmic recycling occurs in a series of ordered steps mediated by cytoplasmic release factors. Failures in these processes can cause both, nuclear biogenesis and export defects due to the lack of the assembly or transport factors in the nucleus.

The cytoplasmic maturation of the pre-60S subunit involves the formation of the ribosomal stalk, incorporation of ribosomal proteins and the release of shuttling biogenesis and export factors (Panse and Johnson, 2010). A comprehensive study from Lo et al. (2010) suggested the following chronology of pre-60S biogenesis steps (Figure 4). 

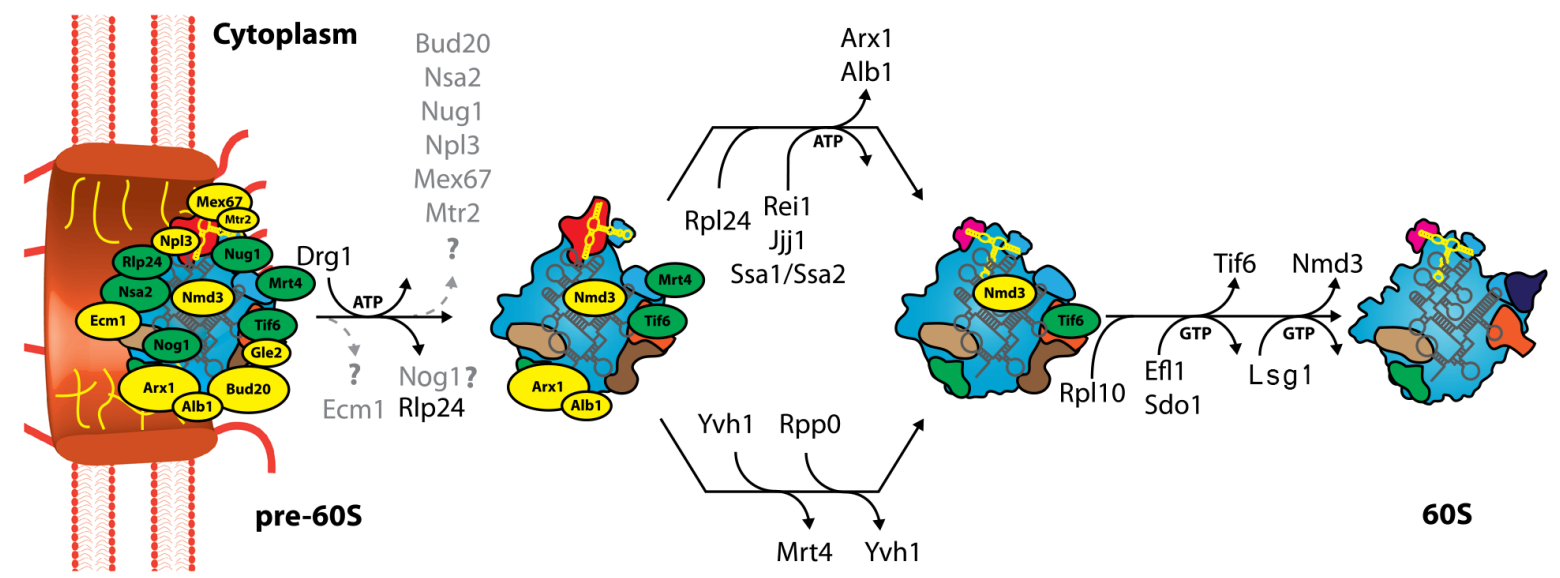

Figure 4: Cytoplasmic maturation of the pre-60S subunit.

The shuttling biogenesis (green) and export factors (yellow) are released from the pre-60S subunit in the cytoplasm by sequential recycling events as schematically indicated. The recycling mechanisms of the factors written in gray are unknown. Slightly modified from Gerhardy et al. (2014).

Besides the export factors, the shuttling biogenesis factors Rlp24, Tif6, Nog1, Nug1, Nsa2 and Alb1 accompany the pre-60S subunit into the cytoplasm (Figure 4) (Gerhardy et al., 2014). The first cytoplasmic maturation step is performed by the AAA-ATPase Drg1, which releases the ribosome-like protein Rlp24 from the pre-60S particles and its action is a prerequisite for the following recycling steps (Kappel et al., 2012; Pertschy et al., 2007). Thus, aberrant pre-60S particles, which are still associated to shuttling export and biogenesis proteins such as Rlp24, Nog1, Arx1 and Tif6 and lack late joining biogenesis factors like Rei1, accumulate in the cytoplasm of temperature-sensitive drg1-18 cells (Kappel et al., 2012; Pertschy et al., 2007). Successful dissociation of Rlp24 allows binding of the ribosomal protein Rpl24 and recruitment of the Zn-finger proteins Rei1 and Yvh1 (Gerhardy et al., 2014). Rei1 together with Jjj1 and the ATPase Ssa1/Ssa2 releases the transport factor Arx1 and its interaction partner Alb1. The ribosome-like protein Mrt4 is dissociated by Yvh1 leading to the assembly of the ribosome stalk, which is necessary for the binding and activation of the elongation factors during translation. Afterwards, the GTPases Efl1 and Sdo1 recycle Tif6 (human elF6), which prevents the premature joining of the immature subunits. Finally, the adaptor protein Nmd3 is released by the action of the GTPase Lsg1/Kre35 and the ribosomal protein Rpl10 (Hedges et al., 2005; West et al., 2005). The incorporation of Rpl10 into the 60S subunit is coupled to the Nmd3 dissociation and is a prerequisite for the subunit joining competence of the large ribosomal subunit. After this step, the maturation of the $60 \mathrm{~S}$ subunit is completed and it is able to facilitate translation. However, the recycling mechanisms for the shuttling export factors Mex67-Mtr2, Bud20, Npl3 and Ecm1 and the biogenesis factors Nog1, Nug1 and Nsa2 are still unknown (Figure 4) (Gerhardy et al., 2014). 
In contrast to the maturation of the pre-60S subunit, less is known about the cytoplasmic biogenesis of the small subunit. In addition to the recycling of bound biogenesis and export factors, this process involves also the processing of the immature 20S pre-rRNA into the mature 18S rRNA, a quality control step and conformational rearrangements of the particle (Panse and Johnson, 2010). The cytoplasmic processing of the $20 \mathrm{~S}$ pre-rRNA begins with the di-methylation of two adenine bases by the methylase Dim1 (Gerhardy et al., 2014). Subsequently, the $20 \mathrm{~S}$ rRNA is cleaved at site $D$ into the mature 18S rRNA (Figure 1) by the endonuclease Nob1, which additionally requires the function of the ATPases Prp43, Rio2 and Fap7.

Seven stably bound assembly factors, which might also contribute to the export, accompany the pre-40S subunit into the cytoplasm and prevent premature translation initiation by blocking the binding of translation initiation factors, the joining of the $60 \mathrm{~S}$ subunit and the opening of the mRNA channel. These factors include the methylase Dim1, the endonuclease Nob1 and its co-factor Pno1/Dim2, the kinase Rio2, the GTPase-like Tsr1, the potential export adaptor Ltv1 and its binding partner Enp1 (Figure 5) (Strunk et al., 2011). How these shuttling biogenesis factors are released from the particle and recycled back to the nucleus is still unclear (Gerhardy et al., 2014). 


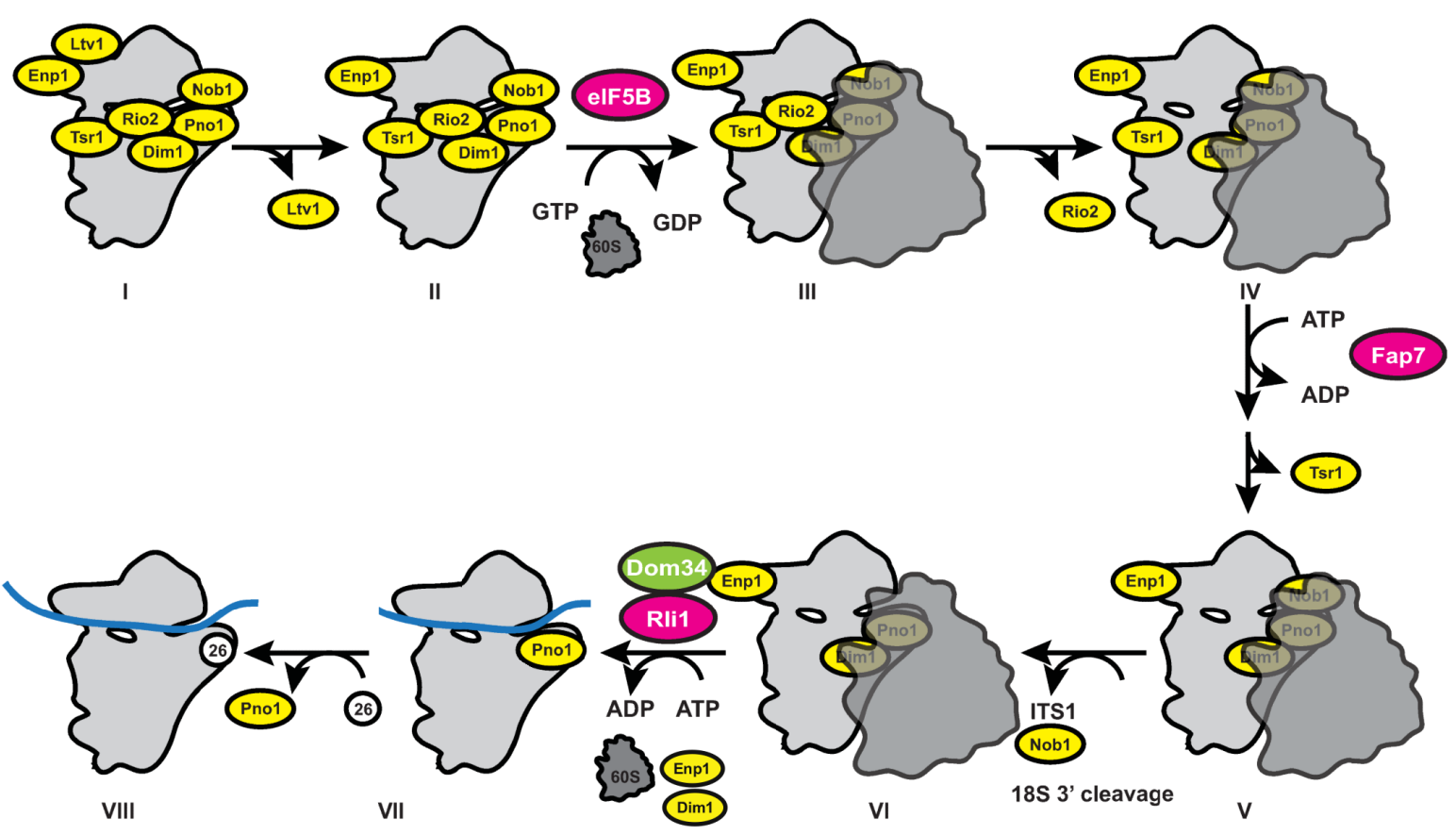

Figure 5: Cytoplasmic maturation and quality control of the pre-40S subunit.

Seven shuttling assembly factors (in yellow) accompany the pre-40S subunit (in light gray) into the cytoplasm (I) and are subsequently released as indicated. The maturation involves the elF5B/ Fun12 dependent joining of the 60S subunit (in dark gray) (II) and the formation of 80S-like ribosomes (III-VI) as a quality control step. The ATPase Fap7 acts (IV) before cleavage of the 20S rRNA by Nob1 leading to mature 18S rRNA (V). Splitting of the 80S-like ribosomes is mediated by Dom34 and Rli1 $(\mathrm{VI})$. The last recycling steps are the release of Pno1 and the incorporation of Rps26, which can occur upon translation initiation (VII). The mRNA is indicated in blue. Modified from Karbstein (2013).

However, Strunk et al. (2012) suggested a serial order for their dissociation based on sucrose-density gradient centrifugation experiments with various yeast mutants (Figure 5). Ltv1 is released before and Rio2 directly after the pre-40S subunits join mature 605 subunits to form 80S-like ribosomes, a process that depends on the GTPase elF5B/Fun12 as during translation initiation. These 80S-like ribosomes are believed to be quality control checkpoints to confirm the translation-competence of the subunits. They contain still $20 \mathrm{~S}$ pre-rRNAs and are mRNA-free. The ATPase Fap7 needs to act on the 80S-like ribosomes and contributes to the $20 \mathrm{~S}$ processing, but its exact function is unclear yet. Nevertheless, Tsr1 is dissociated from the pre$40 \mathrm{~S}$ subunits following the action of Fap7, which seems not to directly displace Tsr1. Successful joining and release of Tsr1 allow the entry of Dom34 and Rli1, which mediate the re-separation of both ribosomal subunits after quality control (see section 4.3.3). The cleavage of the $20 \mathrm{~S}$ pre-rRNA by Nob1 is triggered by the formation of the 80S-like ribosomes and either occurs on these particles or immediately upon their splitting on the pre-40S subunits. Subsequently, Dim1, Nob1, Enp1 and Pno1 are dissociated, whereby the Pno1 release finally allows the incorporation of Rps26 to 
generate mature $40 \mathrm{~S}$ subunits (Strunk et al., 2012). These mature ribosomal subunits are now competent for translation. Interestingly, as for the pre-60S subunits, the recycling mechanism of Mex67-Mtr2 from the pre-40S subunits is unknown.

\subsection{Eukaryotic translation termination and ribosome recycling}

The translation process involves the following steps: the cap-dependent initiation and joining of the ribosomal subunits at the start codon, elongation of the polypeptide chain by the $80 \mathrm{~S}$ ribosomes, translation termination at the stop codon and subsequent recycling of the ribosomal subunits.

\subsubsection{Translation termination}

In general, translation termination takes place upon arrival of the elongating ribosome at the stop codon (UAA, UAG or UGA). This process involves stop codon recognition at the ribosomal A-site and subsequent hydrolysis of the P-site peptidyl-tRNA that leads to peptide release (Jackson et al., 2012). The ester bond between the tRNA and the polypeptide chain is hydrolyzed by the nucleophilic attack of a water molecule in the peptidyl-transferase center (PTC) of the large ribosomal subunit (Song et al., 2000). In all organisms, release factors are necessary to mediate translation termination. These are divided into two classes: class I release factors decode the stop codon and trigger the peptidyl-tRNA hydrolysis and class II factors are supporting ribosome-dependent GTPases (Jackson et al., 2012). However, the bacterial compared to the eukaryotic release factors are evolutionary unrelated and independently developed from each other (Dever and Green, 2012). These proteins differ in their sequences and also in their architectures, but interestingly, all class I release factors contain the universally conserved GGQ motif that is essential for the induction of the peptide release (Song et al., 2000). Positioning of the GGQ motif in the PTC causes a structural rearrangement of the rRNA that allows the entry of a water molecule for the nucleophilic attack of the ester bond in the PTC leading to the hydrolysis of the peptidyl-tRNA (Taylor et al., 2012).

Eukaryotic translation termination is mediated by the eukaryotic release factors eRF1 and eRF3, which are encoded by the essential SUP45 and SUP35 genes in S. cerevisiae (von der Haar and Tuite, 2007). However, in the last years, further factors have been described to be necessary for efficient translation termination in 
addition to the canonical release factors eRF1 and eRF3: the ATP-binding cassette protein Rli1 (Khoshnevis et al., 2010), the DEAD-box RNA-helicase Dbp5 (Gross et al., 2007) and its co-factors Gle1 and $\mathrm{IP}_{6}$ (Alcazar-Roman et al., 2010; Bolger et al., 2008). Recently, the translation initiation factors Hcr1 and elF3 were suggested to influence the termination process as well revealing a tight connection between translation initiation and termination (Beznoskova et al., 2013).

\subsubsection{The eukaryotic release factors eRF1 and eRF3}

The eukaryotic release factor eRF1 is the only class I release factor in eukaryotes and recognizes all three termination codons in contrast to bacteria, which contain two release factors RF1 and RF2 for the decoding of different stop codons (Jackson et al., 2012). Even though the existence of termination factors and nonsense codons were anticipated for long time, the biochemical identification of a protein family with release factor activity including the yeast protein Sup45 was not successful before the 1990s (Frolova et al., 1994; Inge-Vechtomov et al., 2003).

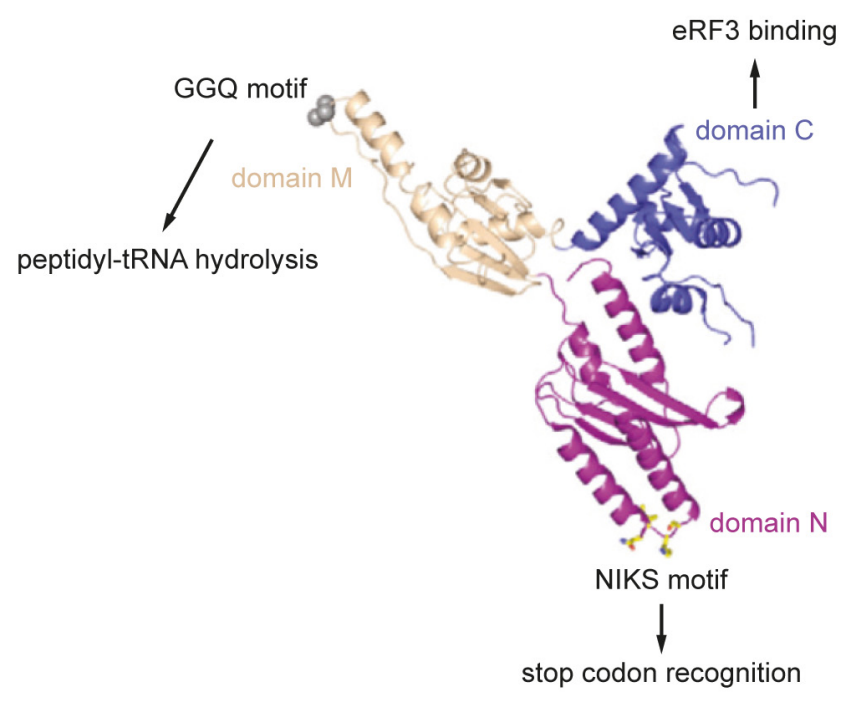

Figure 6: Structure and domains of the eukaryotic release factor eRF1.

The ribbon structure of human eRF1 is shown. The different domains with their important motifs and functions are indicated. Modified from Jackson et al. (2012).

The structure of eRF1/Sup45 mimics a tRNA molecule and consists of three domains (Figure 6) (Ito et al., 1996; Song et al., 2000; Taylor et al., 2012). The N-terminal (N) domain is necessary for stop codon recognition, the middle $(M)$ domain with the conserved GGQ motif mediates the peptidyl-tRNA hydrolysis and the C-terminal (C) domain interacts with the class II release factor eRF3.

The $\mathrm{N}$ domain of eRF1 alone is sufficient for the recognition of all three stop codons in the ribosomal A-site and requires its conserved TASNIKS and YxCxxxF motifs 
(Dever and Green, 2012; Kryuchkova et al., 2013). In total, 15 amino acid residues that are structurally located in close proximity in the $\mathrm{N}$ domain of human eRF1 were defined by Kryuchkova et al. (2013) to be critical for the decoding of stop codons. In addition to that, the 18S rRNA itself might be involved in the discrimination of the second and third position of the stop codon by direct interactions with these nucleotides according to the eRF1 binding mode. The authors suggested the two step model of stop codon recognition. Initially, the first and second nucleotides of the stop codon are decoded by the $N$ domain of eRF1. Subsequently, the rotation of eRF1 within the ribosome enables the recognition of the second and third position of the stop codon (Kryuchkova et al., 2013; Preis et al., 2014). Upon successful stop codon decoding, eRF1 mediates the peptidyl-tRNA hydrolysis in the PTC.

Even though sole eRF1 is able to facilitate peptide release in vitro, the class II eukaryotic release factor eRF3 is required for efficient translation termination in vivo (von der Haar and Tuite, 2007). Initially, the Sup35-like protein from Xenopus laevis was biochemically identified as eRF3 by Zhouravleva et al. (1995). The GTPase eRF3 and its ribosome- and eRF1-stimulated GTP-hydrolysis strongly activates the eRF1 mediated peptide release (Alkalaeva et al., 2006; Frolova et al., 1996; SalasMarco and Bedwell, 2004). In contrast to the bacterial RF3, the GTPase activity of eRF3 is dispensable for the recycling of eRF1 from the ribosome (Nurenberg and Tampe, 2013; Salas-Marco and Bedwell, 2004).

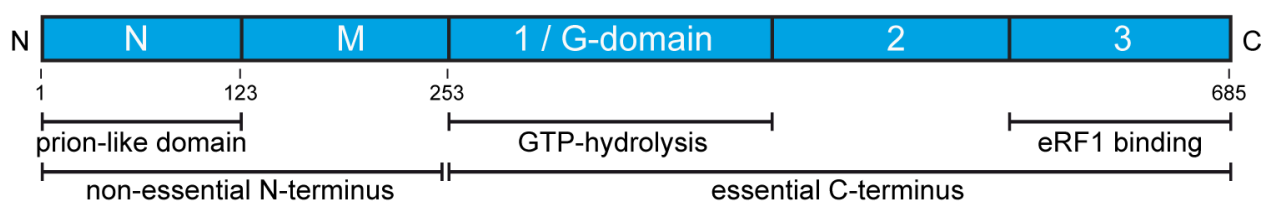

Figure 7: Domain organization of yeast eRF3.

The domains of eRF3 from S. cerevisiae are schematically shown and their particular functions are indicated. The numbering represents the amino acids in the yeast protein. Adapted from Kong et al. (2004).

The eRF3 protein is divided into two major regions, the conserved and for translation termination essential C-terminus and the dispensable N-terminus (Figure 7) (Jackson et al., 2012). The C-terminal region contains the domain 1 or $\mathrm{G}$, which is responsible for GTP-binding and shows similarities to the GTPase fold of the bacterial RF3 or the elongation factor eEF1a (Kong et al., 2004). Further regions of the C-terminus are domains 2 and 3 , which contribute to the interaction with eRF1. In contrast to the essential C-terminus, the non-conserved N-terminal region of eRF3 is dispensable for the termination process and the viability of yeast cells (Ter-Avanesyan et al., 1993). 
However, this region is required for the interaction with the poly(A)-binding protein Pab1 (Hoshino et al., 1999; Roque et al., 2014; Uchida et al., 2002). In yeast, the Nterminus is further divided into the $\mathrm{N}$ and $\mathrm{M}$ domain, whereby the $\mathrm{N}$ region is responsible for the formation of prion-like structures in [PSI $\left.{ }^{+}\right]$strains (Paushkin et al., 1997). In such strains, more than $90 \%$ of the eRF3 molecules were found in aggregated prions that cause a decreased translation termination efficiency (von der Haar and Tuite, 2007). In mammalians, two different long versions of the N-terminus exist and the variant molecules are called eRF3a (GSPT1) and eRF3b (GSPT2) (Hoshino et al., 1998).

The eukaryotic release factors eRF1 and eRF3 strongly interact with each other in vivo and in vitro (Stansfield et al., 1995; Zhouravleva et al., 1995). This interaction is mainly mediated by their $\mathrm{C}$-terminal domains, which represents the domain 3 of eRF3 (Figure 7) (Ebihara and Nakamura, 1999; Eurwilaichitr et al., 1999; Ito et al., 1998; Merkulova et al., 1999; Paushkin et al., 1997). However, the M domain of eRF1 establishes an additional contact to the C-terminus of eRF3 in its GTP-bound form and this interaction is necessary for the stimulation of the GTP hydrolysis (Cheng et al., 2009; Kononenko et al., 2008). While eRF1 stimulates the GTP-binding of eRF3, both, the $80 \mathrm{~S}$ ribosome and eRF1 are needed to activate its GTPase activity (Frolova et al., 1996). Kinetic analyses showed that human eRF3 alone has a higher affinity to GDP than to GTP and binding of eRF1 and eRF3 can occur independent of the presence of a guanine-nucleotide (Pisareva et al., 2006). However, eRF1-bound eRF3 strongly increases the affinity of eRF3 for GTP by inducing a conformational rearrangement in its $\mathrm{G}$ domain that allows the binding of GTP and $\mathrm{Mg}^{2+}$ (Cheng et al., 2009; Kong et al., 2004; Pisareva et al., 2006). These data indicate that cytoplasmic eRF3 is present either alone in its GDP-bound form or as the ternary complex eRF1eRF3-GTP. Most of the current termination models propose that eRF1 and eRF3GTP enter the A-site of the stop codon arrested ribosome as a ternary complex, due to the fact that both proteins interact strongly with each other (Alkalaeva et al., 2006; Jackson et al., 2012; Shoemaker and Green, 2011). However, it is not clear yet, in which form and order the release factors are recruited. In particular, decreased eRF3 levels do not alter the ribosomal binding of eRF1 in yeast cells suggesting that their complex formation is not a prerequisite for the association with the ribosome (SalasMarco and Bedwell, 2004). Contrary to eRF1, the 80 S ribosome does not influence the guanine-nucleotide binding of eRF3 or even the GDP/GTP exchange (Pisareva et al., 2006). So far, no specialized guanine exchange factor (GEF) is known for eRF3. 
However, yeast eEF1B, which is the GEF of elongation factor eEF1A, was found to influence the termination efficiency and might support the guanine-nucleotide exchange on eRF3 (Valouev et al., 2009).

In summary, eRF1 and eRF3 are interdependent for efficient translation termination: eRF1 is needed for the GTP-binding and -hydrolysis of eRF3 and the GTPase activity of eRF3 is required for the eRF1 mediated peptide release (Jackson et al., 2012). Thus, eRF1 and the GTPase eRF3 act cooperatively to facilitate stop codon recognition and peptide release - processes that are accompanied by multiple conformational rearrangements of the release factors and the termination complex (Alkalaeva et al., 2006).

In addition to their functions during translation termination, eRF1 and eRF3 act also in the mRNA quality control pathway the nonsense-mediated decay (NMD), which recognizes and degrades mRNAs with premature termination codons (von der Haar and Tuite, 2007).

\subsubsection{Model for the eRF1 and eRF3 mediated translation termination}

According to the present scientific knowledge, the following model of translation termination was suggested (Figure 8). This model is corroborated by recent cryo-EM structure analyses of pre-termination complexes (des Georges et al., 2014; Preis et al., 2014; Taylor et al., 2012) and their comparison with crystal structures of free eRF1 and/or eRF3 (Cheng et al., 2009; Kong et al., 2004; Song et al., 2000), but misses the new translation termination factors. 

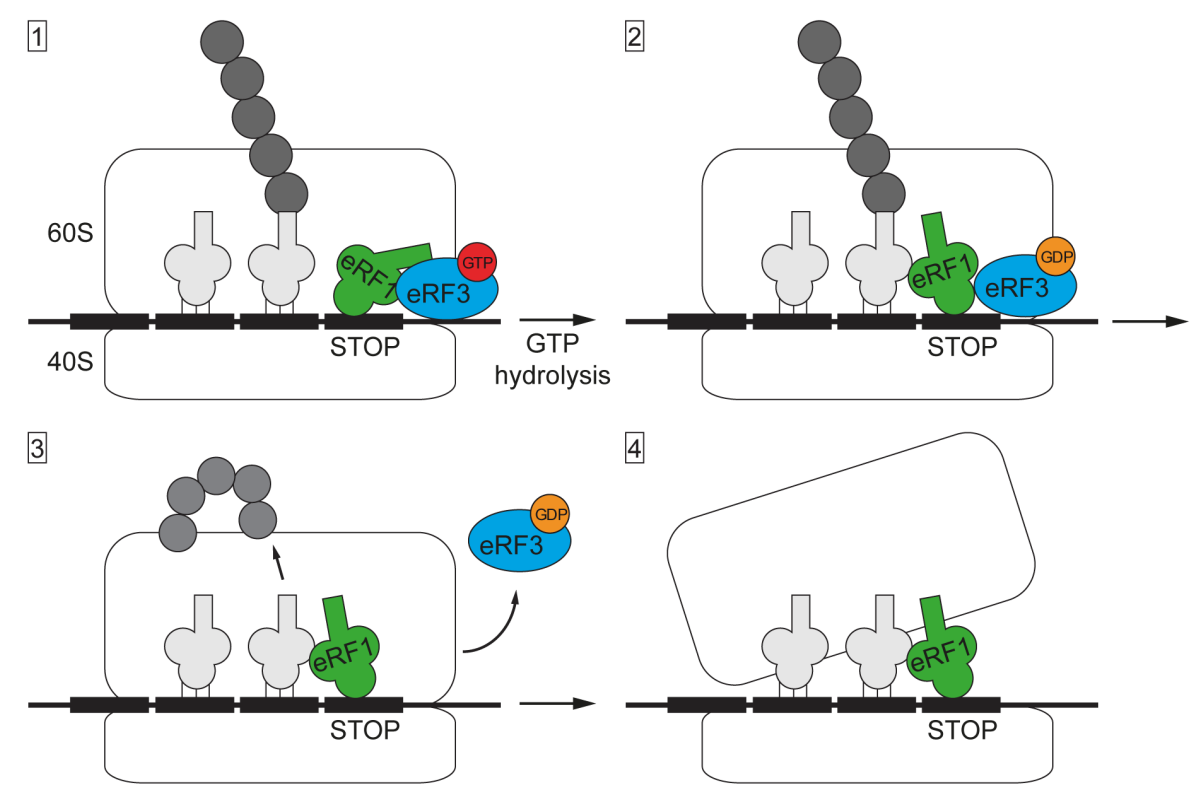

Figure 8: Model of the eRF1 and eRF3 mediated translation termination.

Upon arrival of the ribosome at the stop codon, the tRNA-shaped eRF1 and eRF3-GTP, possibly as ternary complex, enter the ribosomal A-site and eRF1 recognizes the stop codon (1). The ribosome- and eRF1-stimulated GTP-hydrolysis of eRF3 leads to proper positioning of eRF1 in the peptidyl-transferase center (2). Subsequently, eRF1 is able to facilitate peptidyl-tRNA hydrolysis and thus, mediates the peptide release, while eRF3-GDP might dissociate from the ribosome (3). Upon release of the polypeptide chain, the ribosomal subunits are split and all factors are recycled (4). The tRNAs are indicated in light gray, the polypeptide chain in dark gray and the mRNA in black. Adapted from Jackson et al. (2012).

Upon arrival of the elongating ribosome at the stop codon, the release factors, possibly as the eRF1-eRF3-GTP ternary complex, are recruited to the ribosomal Asite (Figure 8, step 1). Studies with an in vitro reconstituted eukaryotic translation system revealed that the ribosomal binding of eRF1 and eRF3-GMPPNP induces a $+2 \mathrm{nt}$ forward toe-print shift of the pre-termination complex (Alkalaeva et al., 2006). Thus, their recruitment seems to induce conformational changes of the ribosomal complex. Furthermore, entering the $80 \mathrm{~S}$ ribosome stimulates the GTPase activity of eRF3 (Figure 8, step 2) (Frolova et al., 1996). The ribosomal binding induces the repositioning of the M domain of eRF1, which enables the GTP-hydrolysis by eRF3 and might depend on the successful stop codon recognition (Cheng et al., 2009; des Georges et al., 2014; Taylor et al., 2012). Different cryo-EM structure analyses of eRF1 and eRF3-GMPPNP bound to the ribosome (des Georges et al., 2014; Preis et al., 2014; Taylor et al., 2012) revealed that the conformation of eRF1 in this pretermination complex significantly differs from the crystal structure of single eRF1 (Song et al., 2000). Both release factors adopt a similar conformation as ribosomebound aminoacyl-tRNA/EF-Tu during translation elongation or Dom34/Hbs1 during no-go decay. In these structures, eRF3 binds near the GTPase-associated center of the ribosome and its $\mathrm{C}$-terminal domain 3 interacts with the $\mathrm{C}$-terminal domain of 
eRF1. The $\mathrm{N}$ domain of eRF1 enters the decoding center of the $40 \mathrm{~S}$ subunit for the stop codon recognition, whereas the M domain with the GGQ motif is located in a locked conformation between the $G$ domain of eRF3 and the $60 S$ subunit. In this conformation, the GGQ motif of eRF1 is far away from the peptidyl-transferase center so that it is unable to mediate peptide release. However, the ribosome- and eRF1induced GTP-hydrolysis of eRF3 causes the conformational rearrangement of the $M$ domain of eRF1 and facilitates the proper positioning of the GGQ motif in the PTC (Figure 8, step 2). This conformation allows subsequent peptidyl-tRNA hydrolysis (Figure 8, step 3), as described in section 4.2.1.

\subsubsection{Ribosomal recycling}

Upon release of the polypeptide chain, the post-termination complex needs to be recycled (Figure 8, step 4). The 80 S ribosome, which still binds the mRNA and contains the deacylated tRNA in the P-site and most likely eRF1 in the A-site, is split into the 60S and the mRNA/tRNA-bound 40S subunit (Dever and Green, 2012). While at least eRF1 is required for the recycling process, GDP-bound eRF3 seems to dissociate from the ribosome upon GTP-hydrolysis (Figure 8, step 3) (Pisarev et al., 2010). In contrast to eukaryotes and archaea, bacteria contain the ribosomerecycling factor (RRF) that is recruited to their 70S ribosomes by the GTP-bound elongation factor EF-G (Nurenberg and Tampe, 2013). Subsequent GTP-hydrolysis leads to the RRF-mediated splitting of the $70 \mathrm{~S}$ ribosomes into the subunits. In eukaryotes and archaea, Rli1/ABCE1 with its ATPase activity is needed instead for the ribosomal separation and is recruited upon dissociation of GDP-bound eRF3 or archaeal aEF1a. Upon splitting, the initiation factors elF3, Hcr1, elF1 and elF1A release the tRNA and the mRNA from the $40 \mathrm{~S}$ subunit (Jackson et al., 2012).

\subsection{The iron-sulfur containing ATP-binding cassette protein Rli1}

Rli1 belongs to the superfamily of ATP-binding cassette $(A B C)$ proteins and is the only member of the subfamily $E$, wherefore it is also called ABCE1, usually in human and archaea (Franckenberg et al., 2012). The protein was initially identified as an inhibitor of RNase $L$ in the antiviral response of human cells and was named

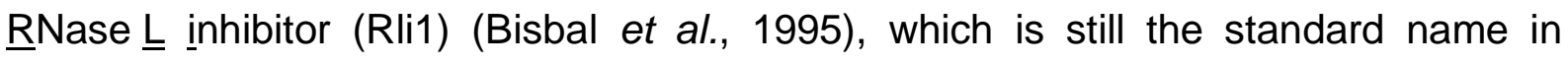
S. cerevisiae. In contrast to most ABC proteins, Rli1 is not a membrane transporter, 
but a soluble protein and predominantly localized in the cytoplasm (Dong et al., 2004; Kispal et al., 2005). However, the molecule can also shuttle between the nucleus and the cytoplasm, as yeast Rli1 accumulates in the nuclei of export defective xpo1-1 cells (Kispal et al., 2005; Yarunin et al., 2005). Despite several other cellular functions that are described, Rli1/ABCE1 is established as the conserved eukaryotic and archaeal ribosome recycling factor (Nurenberg and Tampe, 2013).

\subsubsection{The structure of Rli1}

Rli1 is an iron-sulfur protein, which carries two essential $[4 \mathrm{Fe}-4 \mathrm{~S}]^{2+}$ clusters in the Nterminus (Figure 9A), for which it requires the mitochondrial and cytosolic Fe-S cluster biogenesis machineries (Barthelme et al., 2007; Kispal et al., 2005).

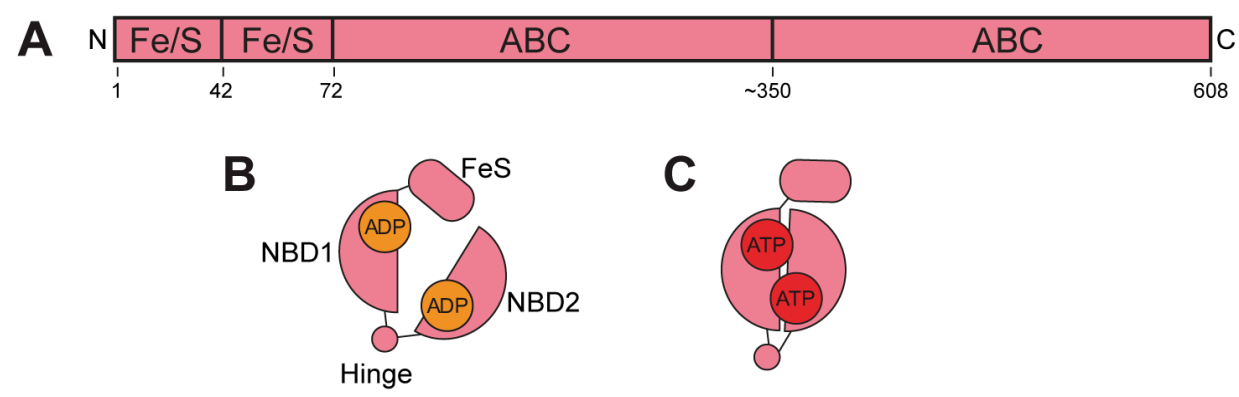

Figure 9: The domain structure and the conformations of Rli1.

(A) The domain structure of Rli1 is schematically shown and the numbering represents the amino acids in the yeast protein. Rli1 consists of two N-terminal iron-sulfur clusters (Fe/S) and two C-terminal ATP-binding cassette (ABC) domains. Modified from Kispal et al. (2005).

(B-C) Scheme of the different conformations of Rli1. (B) Both nucleotide binding domains (NBD1 and NBD2) are linked by the hinge domain and adopt an open conformation in the ADP-bound form. (C) ATP-binding induces closure of the NBDs with a concomitant movement of the iron-sulfur domain (FeS). The pictures are adapted from Becker et al. (2012).

In the C-terminus, two $\mathrm{ABC}$-domains exist that contain the nucleotide binding domains (NBDs) for the binding and hydrolysis of ATP (Figure 9A). Crystal structure analyses of archaeal Rli1 showed that the two NBDs are connected by a unique hinge domain and are arranged in an ABC-protein characteristic head-to-tail orientation with a cleft between them (Barthelme et al., 2011; Karcher et al., 2005; Karcher et al., 2008). The cleft contains the two ATP-binding sites face-to-face and forms the active center of the enzyme (Karcher et al., 2005). During its ATPase cycle, Rli1 adopts different conformations (Figure 9B-C). The gap between the NBDs is open in its ADP-bound form (Figure 9B), while ATP-binding induces a tight closure of the cleft and allows hydrolysis of the ATP molecules (Figure 9C). This ATPdependent tweezers-like motion of the NBDs causes a power stroke, which enables conformational changes in binding partners and converts the chemical energy of the 
ATP-binding and hydrolysis into mechanical work, such as it is typical for ABCproteins (Becker et al., 2012; Chen et al., 2003; Karcher et al., 2005). Furthermore, the iron-sulfur domain is located at the opening of the cleft and has contact to the active site of NBD1 (Figure 9B) (Karcher et al., 2008). Thus, the ATP-driven conformational change from the open to the closed state leads to a concomitant motion of the iron-sulfur domain (Figure 9C) (Karcher et al., 2005; Karcher et al., 2008). This movement is important for the transfer of the mechanochemical power to interaction partners such as eRF1 or Dom34 during ribosome splitting (Barthelme et al., 2011; Becker et al., 2012).

Rli1 is essential in all so far tested organisms and mutation of conserved cysteine residues in the Fe-S clusters, or of amino acids that are necessary for the binding and hydrolysis of ATP in the ABC-domains are inviable (Barthelme et al., 2007; Dong et al., 2004; Karcher et al., 2005; Kispal et al., 2005). These data reveal the importance of all three domains, the iron-sulfur and both $A B C$ domains for the functionality of Rli1.

\subsubsection{The diverse functions of Rli1}

Rli1 does not exist in bacteria, but the protein is highly conserved in eukaryotes and archaea (Chen et al., 2006; Kispal et al., 2005). Thus, a fundamental and conserved function of the enzyme would be expected and several cellular roles were already suggested. Initially, Rli1 was found to act in the antiviral response of human cells by inhibition of the RNase L (Bisbal et al., 1995) and Rli1 is also involved in the formation of the HIV-1 capsid (Zimmerman et al., 2002). However, these specialized roles in higher eukaryotes or for viruses cannot be conserved functions of the protein, but rather its contribution to translational processes. Besides translation termination and ribosomal recycling, Rli1 might also be involved in translation initiation (Chen et al., 2006; Dong et al., 2004). Dong et al. (2004) showed that yeast Rli1 co-sediments predominantly with pre-initiation complexes and interacts with the initiation factors elF5 and subunits of elF2 and elF3 of the yeast multifactor complex (MFC) in vivo. Depletion of RLI1 leads to a decreased binding of MFC components to 40S subunits suggesting that functional Rli1 is necessary for the formation of 435 pre-initiation complexes. Furthermore, the protein synthesis is reduced and a polysome run-off is visible (Dong et al., 2004; Kispal et al., 2005). Similar results were obtained for human ABCE1 showing the conservation of this function (Chen et al., 2006). According to a role in translation initiation, most of the Rli1 molecules are bound to 
$40 \mathrm{~S}$ subunits, but parts are also associated with $80 \mathrm{~S}$ ribosomes and polysomes in sucrose-density gradients analyses, which indicates further cellular functions for Rli1 (Dong et al., 2004; Kispal et al., 2005; Yarunin et al., 2005). Interestingly, Rli1 predominantly interacts with the non-essential and transiently associated elF3 subunit Hcr1/elF3j (Khoshnevis et al., 2010; Kispal et al., 2005; Yarunin et al., 2005), which is besides its function in translation initiation also involved in translation termination (Beznoskova et al., 2013) and in the final maturation of the 18S rRNA (Valasek et al., 2001). This interaction confirms a role of Rli1 in translation termination and also in the biogenesis and the nuclear export of both pre-ribosomal subunits. For the second function, Rli1 and its Fe-S cluster are necessary, as depletion of RLI1 or of components of the cytosolic Fe-S cluster assembly machinery cause the nuclear accumulation of the pre-60S reporter Rpl25-GFP and the pre-40S marker Rps2-GFP (Kispal et al., 2005; Yarunin et al., 2005). Furthermore, rRNAprocessing defects in the synthesis of both, the large and the small ribosomal subunits were visible in Northern blot analyses of RLI1 depleted cells (Yarunin et al., 2005). In fact, Rli1 binds to late $20 S$ and 75 pre-rRNAs, but no interactions with intermediates of earlier biogenesis pathways were detected, suggesting an association with late pre-40S and pre-60S subunits (Figure 1) (Yarunin et al., 2005). In accordance with these results, a function of Rli1 in a quality control step during cytoplasmic maturation of ribosomal subunit was proposed by Strunk et al. (2012), as described in section 4.1.2. Thus, Rli1 might be needed as ribosome recycling factor during ribosomal biogenesis.

\subsubsection{Rli1 in translation termination and ribosome recycling}

In the last years, several groups showed that Rli1/ABCE1 is required for the recycling of ribosomes upon translation termination and this function is conserved among eukaryotes and archaea (Nurenberg and Tampe, 2013). Experiments with an eukaryotic in vitro reconstituted translation system revealed that $A B C E 1$ promotes the dissociation of $80 \mathrm{~S}$ ribosomes into $60 \mathrm{~S}$ and mRNA/tRNA-bound $40 \mathrm{~S}$ subunits, when translation termination and peptide release was mediated by eRF1 and eRF3 (Pisarev et al., 2010). It was shown before that the ribosomal splitting can also be triggered by the initiation factors elF3, elF1 and elF1A, but only at low $\mathrm{Mg}^{2+}$ concentrations in vitro (Pisarev et al., 2007). However, eukaryotic ABCE1 with its ATPase activity is required for efficient ribosomal recycling at varying $\mathrm{Mg}^{2+}$ concentrations and this process depends on the presence of eRF1 (Pisarev et al., 
2010). Similarly, the archaeal ABCE1 mediates also the ribosome separation and the dissociation of the archaeal release factor aRF1 upon translation termination, for which solely its adoption of the ATP-bound closed conformation and the concomitant movement of the iron-sulfur domain seems to be necessary (Barthelme et al., 2011). Subsequently, the archaeal ABCE1 itself might be released from the small ribosomal subunit by its ATP-hydrolysis. However, this model stays in contrast to other publications showing that the ATP-hydrolysis of yeast and human Rli1/ABCE1 is also required for successful subunit splitting (Pisarev et al., 2010; Shoemaker and Green, 2011). Thus, it is possible that differences in the mode of action exist between archaea and eukaryotes.

Furthermore, Rli1 does not only mediate the ribosome recycling after translation termination, but also the splitting of empty or stalled ribosomes together with the eRF1 and eRF3 paralogues Dom34 (human Pelota) and Hbs1 during quality control steps (Pisareva et al., 2011; Shoemaker and Green, 2011; Strunk et al., 2012; van den Elzen et al., 2014). Thus, peptide release is not a prerequisite for the separation of ribosomal subunits by Rli1, but the presence of Dom34/Hbs1 or the canonical release factors eRF1/eRF3 (Pisarev et al., 2010; Pisareva et al., 2011; Shoemaker and Green, 2011).

From the establishment of Rli1 as a general ribosome recycling factor arises the question whether the initiation defects detected in RLII depleted cells might also result from ribosomal recycling or re-initiation defects (Pisarev et al., 2010).

Interestingly, in addition to its function in ribosome splitting, Rli1 is also required earlier during translation termination (Khoshnevis et al., 2010). The authors showed that yeast Rli1 physically and genetically interacts with both release factors eRF1 and eRF3 in vivo. However, the interaction with eRF1 was more intense (Khoshnevis et al., 2010) that might explain the missing association between Rli1 and eRF3 in the co-immunoprecipitation experiments of Dong et al. (2004). Furthermore, in vitro binding studies detected for recombinant Rli1 a direct binding to eRF1 and Hcr1, which is mediated by the C-terminal ABC-domain of Rli1 (Khoshnevis et al., 2010). Similarly, human and archaeal ABCE1 also interacts directly and stoichiometrically with eRF1 and aRF1, respectively (Barthelme et al., 2011; Pisarev et al., 2010). Moreover, this binding did not dependent on the iron-sulfur domain (Barthelme et al., 2011), which stays in contrast to cryo-EM structure analyses that revealed a contact between the C-terminal domain of eRF1 and the iron-sulfur domain of yeast and archaeal Rli1 bound to the ribosome (Preis et al., 2014). This data indicate that the 
interaction domain might change in the absence of the ribosome.

Sole binding to the release factors does not confirm a direct function of Rli1 in the termination process, as the interaction could also take place during ribosome recycling. However, depletion of yeast RLI1 causes also defects in the recognition of stop codons and leads to an increased read-through activity in a dual reporter assay indicating a role of Rli1 in translation termination (Khoshnevis et al., 2010). Furthermore, overexpression of wild type RLI1, but not of the Fe-S cluster defective rli1(C28S), partially rescues the high read-through activity of the eRF1 mutant sup45-2 (Khoshnevis et al., 2010). These data suggest that the Fe-S domain is necessary for the function of Rli1 during translation termination. Studies with an in vitro reconstituted S. cerevisiae translation system revealed that Rli1 accelerates ATPase independently the rate of peptide release by eRF1, whereas efficient ribosome splitting depends on the ATP-hydrolysis by Rli1 (Shoemaker and Green, 2011).

Additional insights into the mechanism of how Rli1 can operate during translation termination and ribosome recycling arose from cryo-EM structure analyses of archaeal and yeast Rli1 bound to the eRF1- or Dom34-containing ribosomes (Becker et al., 2012; Preis et al., 2014). These structures revealed that Rli1 binds in a halfopen conformation in the intersubunit space and contacts especially the small, but also the large ribosomal subunit. Furthermore, the Fe-S domain of Rli1 associates with the C-terminal domains of eRF1 or Dom34. This position is the same ribosomal binding site as that of the GTPases eRF3 or Hbs1 and therefore, binding of Rli1 must occur upon their dissociation. According to such mutually exclusive binding, it was shown previously that GTP-hydrolysis of eRF3/Hbs1 and their release is a prerequisite for the action of Rli1 (Pisareva et al., 2011; Shoemaker and Green, 2011). Recently, the translation initiation factor Hcr1 was suggested to stimulate the dissociation of eRF3-GDP from the ribosome upon its GTP-hydrolysis allowing the entry of Rli1 (Beznoskova et al., 2013). Furthermore, addition of eRF3 and the nonhydrolysable GTP analog GMPPNP leads to a decreased binding of Rli1 to eRF1bound ribosomes (Pisarev et al., 2010). Thus, the authors suggested that the premature association of Rli1 with the terminating ribosome is prevented by eRF3GTP.

The conformation of Rli1-bound eRF1 or Dom34 in these termination complexes differ compared to their eRF3-GTP or Hbs1-GTP bound forms in the pre-termination complexes, where their central domains are locked between the $G$ domain of 
eRF3/Hbs1 and the 60S subunit (Becker et al., 2012; Preis et al., 2014). By this conformational switch, the region, which contains the essential GGQ motif in eRF1, is perfectly positioned in the peptidyl-transferase center to mediate the peptide release. Thus, Rli1 might support translation termination by locking this favorable conformation after eRF3-GDP dissociation, as also previously suggested by Shoemaker and Green (2011).

For its function in ribosome recycling, Rli1 has to undergo a conformational change from its half-open to the completely closed state, which might be induced by the ribosomal binding (Becker et al., 2012). It is not clear yet, which nucleotide is bound in the half-open state. However, the authors suggested that the ribosome-binding might increase the affinity of Rli1 for ATP. The ATP-binding causes the tight closure of the NBDs that allows ATP-hydrolysis and is accompanied by the movement of the Fe-S domain (Becker et al., 2012; Franckenberg et al., 2012).

In summary, based on the presented data, the following model for translation termination and ribosome recycling mediated by Rli1 can be suggested (Figure 10). The different steps are ordered by several NTP-hydrolysis events, as described by Shoemaker and Green (2011).

Pre-termination

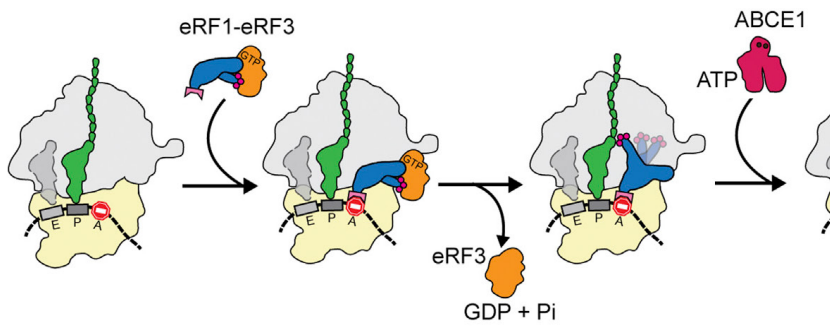

Termination

Pre-recycling

Figure 10: Model for translation termination and ribosome recycling mediated by Rli1.

Upon arrival of the $80 \mathrm{~S}$ ribosome at the stop codon, eRF1 (in blue) and eRF3-GTP (in orange) are recruited to the ribosomal A-site and eRF1 recognizes the stop codon. GTP-hydrolysis of eRF3 leads to the proper positioning of eRF1 in the peptidyl-transferase center and to dissociation of eRF3-GDP. Subsequently, ATP-bound ABCE1/Rli1 (in red) is able to bind at the same binding site and locks eRF1 in the favorable position for hydrolysis of the peptidyl-tRNA (in green). Upon release of the polypeptide chain, Rli1 mediates the ATPase-dependent splitting of the ribosomal subunits. Modified from Preis et al. (2014).

Upon arrival of the ribosome at the stop codon, eRF1 and eRF3-GTP enter the ribosomal A-site and successful stop codon recognition by eRF1 stimulates the GTPase activity of eRF3. The GTP-hydrolysis causes a conformational rearrangement of eRF1, which perfectly positions its GGQ motif in the peptidyltransferase center. Upon dissociation of eRF3-GDP, Rli1-ATP, possibly in a half- 
open state, is able to bind and to stabilize the favorable conformation of eRF1. Thus, eRF1 can mediate the hydrolysis of the peptidyl-tRNA and the release of the polypeptide chain. Subsequently, the complete closure of the catalytic center of Rli1 enables its ATP-hydrolysis and the concomitant motion of the Fe-S domain, which facilitates ribosome recycling. The separation of the ribosomal subunits might be mediated by the last conformational change of Rli1, which is transferred by the Fe-S domain to the C-terminal domain of eRF1 and consequently to the ribosome. Upon ribosomal splitting, Rli1 remains associated with the tRNA/mRNA-bound 40 S subunit.

\subsection{The DEAD-Box RNA-helicase Dbp5/Rat8 and its co-factor Gle1}

The $\underline{D} E A D$ - $\underline{b} 0 x$ protein 5 (Dbp5/also called Ribonucleic acid-trafficking protein Rat8 / human DDX19) is a DEAD-box RNA helicase, which remodels RNA-protein complexes during its RNA-dependent ATPase cycle. The essential and conserved protein is well known for its function in the transport of mRNAs from the nucleus into the cytoplasm (Schmitt et al., 1999; Snay-Hodge et al., 1998; Tseng et al., 1998; Zhao et al., 2002). Furthermore, yeast Dbp5 is involved in translation termination in the cytoplasm and it remains to be shown, whether this function is also conserved in higher eukaryotes (Gross et al., 2007). For both of these functions, the stimulation of Dbp5 by the co-factors Gle1 and the small signal molecule inositol 1,2,3,4,5,6hexakiphosphate $\left(\mathrm{IP}_{6}\right)$ is necessary (Alcazar-Roman et al., 2006; Weirich et al., 2006).

\subsubsection{The structure of Dbp5}

Dbp5 is an RNA-binding protein and belongs as a DEAD-box protein to the helicase superfamily 2 , which contain a conserved catalytic helicase core consisting of two structurally similar RecA-like domains and a short flexible linker region between them (Figure 11A) (Linder and Jankowsky, 2011). Both RecA-like domains from a cleft that binds the ATP molecule and need to be closed for ATP hydrolysis, while the RNA binding site is located opposite to the cleft opening (Figure 11B) (Tieg and Krebber, 2013). 

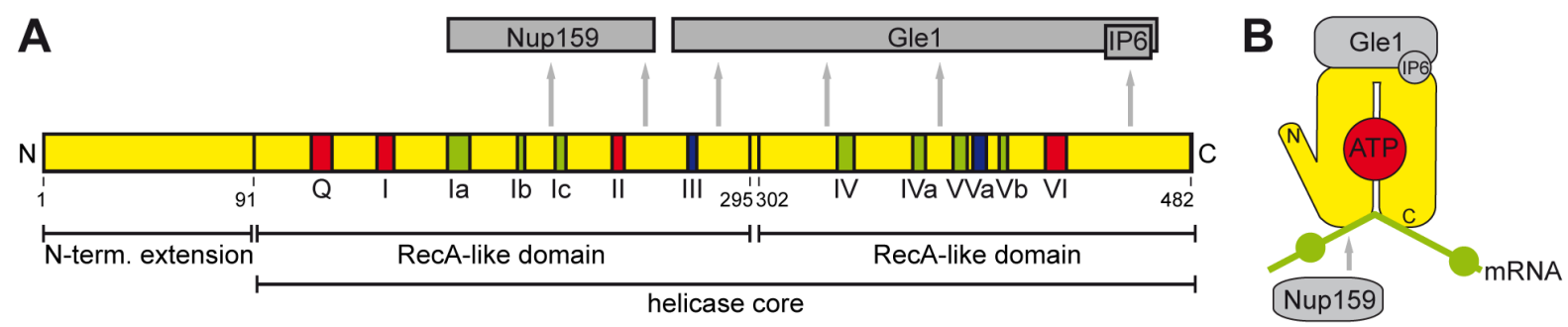

Figure 11: The domain structure of Dbp5 and its binding regions for interaction partners.

(A) A scheme of the domain structure of Dbp5 (in yellow) is shown and the numbers represent the amino acids of yeast Dbp5. The catalytic helicase core consists of two RecA-like domains, which contain the 13 conserved motifs for ATP binding and hydrolysis (in red), for RNA binding (in green) or for both (in blue). Additionally, Dbp5 has a unique N-terminal extension. Regions for interaction with the binding partners Nup159, Gle1 and $\mathrm{IP}_{6}$ are indicated in gray. (B) The structural organization of Dbp5 (in yellow) and the binding sites for its interaction partners are schematically shown. Modified from Tieg and Krebber (2013).

In general, the helicase core of DEAD-box RNA-helicases exhibits 13 characteristic and conserved motifs for the RNA binding and ATP hydrolysis and carries the eponymous Asp-Glu-Ala-Asp (DEAD) sequence in motif II (Figure 11A) (Linder and Jankowsky, 2011). These helicases bind sequence-unspecific the sugar-phosphatebackbone of single-stranded RNAs and fulfill different functions during their ATPase cycles such as remodeling of the bound protein-RNA complexes, unwinding of short duplex RNAs or RNA clamping and recruitment of larger complexes (Linder and Jankowsky, 2011).

In particular, Dbp5 is an DEAD-box RNA-helicase with remodeling activity that displaces RNA-bound proteins during its ATPase cycle and is best characterized for its function in mRNA export (Tieg and Krebber, 2013).

\subsubsection{Dbp5 and Gle1 in nuclear mRNA export}

The transcription and processing of mRNAs is performed in the nucleus. Only completely processed molecules containing a poly(A)-tail at the 3'end and a 5'cap are actively exported through the NPCs into the cytoplasm, where translation of these mRNAs takes place. During their complete life cycles, mRNAs are accompanied by several proteins and form large messenger ribonucleoprotein (mRNP) particles, whose composition constantly changes (Kelly and Corbett, 2009). For the transport, the export receptor heterodimer Mex67-Mtr2 needs to bind to the mRNPs in the nucleus and is predominantly recruited via adapter proteins such as the nuclear Yra1, the shuttling SR-proteins Npl3, Gbp2, Hrb1 or the poly(A)-binding protein Nab2 (Figure 12) (Hackmann et al., 2014; Stewart, 2010). The heterodimer Mex67-Mtr2 interacts with all FG-repeat containing nucleoporins of the NPC channels for the mRNP translocation (Strasser et al., 2000). In the cytoplasm, 
Mex67-Mtr2 and Nab2 dissociate from the exported mRNAs and shuttle back into the nucleus, while other proteins such as the SR-proteins remain bound to the mRNAs until translation (Figure 12) (Windgassen et al., 2004). This compositional change is anticipated to cause a directional transport process, because the diffusion of the translocated mRNAs back into the NPCs is prevented.

In the 1990s, different temperature-sensitive mutants of DBP5 and GLE1 were found to rapidly accumulate poly $(A)$-tail containing mRNAs in the nucleus indicating a function of Dbp5 and Gle1 in nuclear mRNA export (Del Priore et al., 1996; Murphy and Wente, 1996; Snay-Hodge et al., 1998; Tseng et al., 1998).

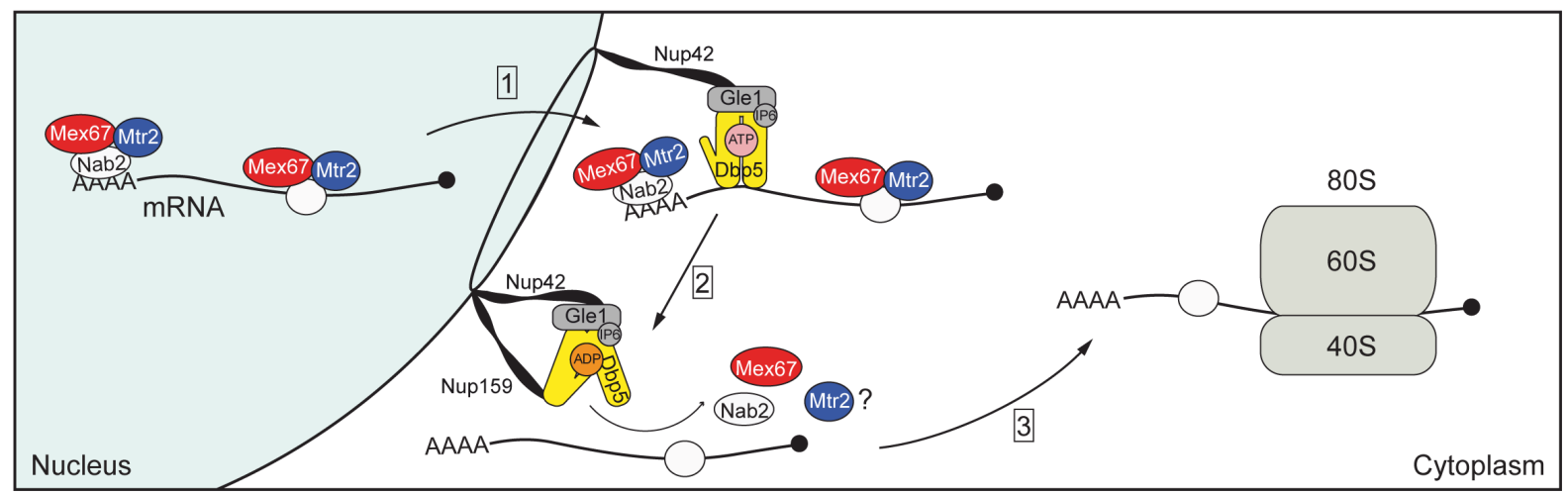

Figure 12: Model for nuclear mRNA export mediated by Dbp5 and Gle1.

In the nucleus, the export receptor heterodimer Mex67-Mtr2 is recruited via adapter proteins (in white) such as Nab2 to export competent mRNAs and facilitates their translocation through the NPCs (1). Dbp5 is localized to the cytoplasmic fibrils of the NPCs and binds the emerging mRNAs. The binding stimulates the Gle1-IP ${ }_{6}$ dependent ATPase activity of Dbp5 leading to the displacement of Nab2, Mex67 and possibly Mtr2 from the mRNA (2). This compositional change causes the directionality of the transport event. Other associated adapter proteins remain bound until translation of the mRNA (3).

Indeed, it was suggested that the DEAD-box RNA-helicase Dbp5 and its stimulation by Gle1 is required for the remodeling of emerging mRNPs at the cytoplasmic sides of the NPCs. Dbp5 displaces during its ATPase cycle the mRNA bound export factors Mex67 and Nab2 leading to the directional mRNA export (Figure 12, step 2) (Lund and Guthrie, 2005; Tran et al., 2007). In vivo experiments in yeast cells showed an increased binding of Mex67 to poly(A)-tail containing mRNAs at the nuclear rim of dbp5 mutants (Lund and Guthrie, 2005). Furthermore, studies with recombinant purified yeast proteins revealed that Dbp5 can dissociate Nab2 from RNAs in vitro (Tran et al., 2007). It remains to be shown, whether Mtr2 is also released from the mRNA by Dbp5, but this scenario is well possible, as Mex67 and Mtr2 form a stable heterodimer.

For the protein displacement, the ATP-hydrolysis of Dbp5 needs to be stimulated by the co-factors Gle1 and $\mathrm{IP}_{6}$ and especially the transition from the closed ATP-bound 
to the open ADP-bound conformation of Dbp5 is critical (Figure 12, step 2) (AlcazarRoman et al., 2006; Noble et al., 2011; Tran et al., 2007; Weirich et al., 2006). However, it is unclear yet, how Dbp5 exactly dissociates the bound proteins from the mRNA and how this RNA-helicase selects the specific proteins during this step. Anyhow, the regulated ATPase cycle of Dbp5 is required for this mRNP remodeling that facilitates the directional mRNA export.

\subsubsection{The regulation of the ATPase cycle of Dbp5 during mRNA export}

Dbp5 is an RNA-binding protein that only transiently binds to single-stranded RNAs in its ATP-bound form, while subsequent ATP-hydrolysis leads to the RNA release (Tran et al., 2007; von Moeller et al., 2009; Weirich et al., 2006). The RNA-binding is necessary for activation of the ATP-hydrolysis of Dbp5 (Schmitt et al., 1999; Tseng et al., 1998). Dbp5 has a unique and flexible N-terminal extension (Figure 11), which mediates this RNA-dependency and thereby self-regulates its ATPase activity (Collins et al., 2009). This domain forms an $\alpha$-helix and prevents premature ATPhydrolysis by separation of the cleft between both RecA-like domains, which is the catalytic center of the helicase. Only upon RNA-binding, the N-terminal extension is removed allowing the complete closure of the helicase core and ATP-hydrolysis. Despite this RNA-dependent self-activation, Dbp5 in general requires for its ATPase activity the stimulation by the co-factors Gle1 and the small signal molecule $\mathrm{IP}_{6}$, which was shown in different in vitro ATPase assays (Alcazar-Roman et al., 2006; Weirich et al., 2006). Dbp5 and Gle1 mainly interact via their C-terminal domains, whereas the N-terminal RecA-like domain of Dbp5 only slightly contributes to the Gle1 binding (Figure 11) (Dossani et al., 2009; Montpetit et al., 2011; Weirich et al., 2006). Their interaction is stabilized by the binding of $\mathrm{IP}_{6}$ to a positively charged pocket between their C-terminal domains (Alcazar-Roman et al., 2010; Montpetit et al., 2011). The exact mechanism of how the complex of Gle1-IP 6 stimulates Dbp5 is still controversially discussed. Montpetit et al. (2011) suggested from structural and biochemical analyses that binding of Gle1-IP 6 to ATP-bound Dbp5 promotes its ATPhydrolysis and enhances RNA-release by stabilization of the open ADP-bound conformation of Dbp5. In this conformation, the RNA-binding site of Dbp5 is altered leading to the RNA displacement, which is the rate-limiting step of the enzyme. In contrast to this model, studies with diverse dbp5 mutants indicated that Gle1-IP 6 stimulates the ATP-binding of Dbp5 as a prerequisite for its RNA association, which subsequently leads to the release of the co-factors and ATP-hydrolysis (Folkmann et 
al., 2011; Hodge et al., 2011; Noble et al., 2011). Thus, further investigations will be needed to uncover the mechanism of Dbp5 activation.

Furthermore, Dbp5 is localized to the cytoplasmic fibrils of the NPCs via binding to the N-terminal domain of the yeast nucleoporin Nup159/Rat7 (Hodge et al., 1999; Schmitt et al., 1999; Snay-Hodge et al., 1998; Weirich et al., 2004) or the human CAN/NUP214, respectively (Napetschnig et al., 2009; Schmitt et al., 1999; von Moeller et al., 2009). Placed there, Dbp5 is ideally localized for its function during nuclear mRNA export. However, Nup159 is not only needed to tether Dbp5 to the cytoplasmic fibrils, but rather regulates its ATPase cycle as an ADP-release factor (Noble et al., 2011). Nup159 interacts with the tip of the N-terminal RecA-like domain of Dbp5, (Figure 11) (Napetschnig et al., 2009; von Moeller et al., 2009; Weirich et al., 2004). This interaction site partially overlaps with the RNA-binding site of Dbp5, so that binding of RNA and Nup159 must be mutually exclusive and Nup159 association occurs upon RNA-release (Montpetit et al., 2011; Napetschnig et al., 2009; von Moeller et al., 2009). The subsequent binding of Nup159 stimulates a conformational change in Dbp5 with a further opening of the helicase core, which allows ADP release and recycling of the enzyme (Montpetit et al., 2011; Noble et al., 2011).

In summary, according to these data, the following regulated ATPase cycle of Dbp5 can be suggested for mRNA export (Figure 13) (Tieg and Krebber, 2013). 


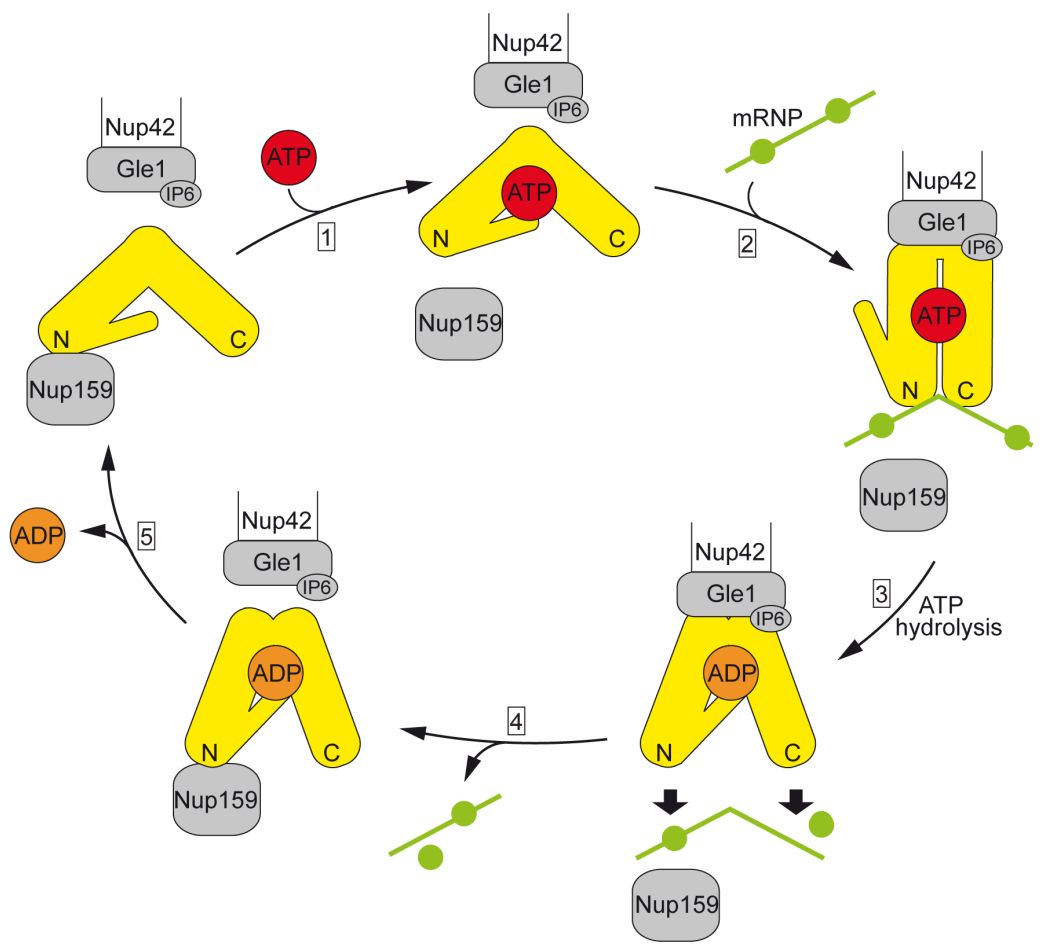

Figure 13: Scheme of the ATPase cycle of Dbp5 during nuclear mRNA export.

Nucleotide-free Dbp5 (in yellow) has an open conformation with separated RecA-like domains and binds first ATP (1). ATP-Dbp5 interacts with the exported mRNP and possibly also with the cofactors $\mathrm{IP}_{6}$ and Gle1, which is tethered by Nup42/Rip1 to the cytoplasmic fibrils (2). This binding removes the $\mathrm{N}$-terminal extension leading to a closed conformation and complete formation of the catalytic center, which is able to hydrolyze ATP (3). The transition into ADP-Dbp5 is accompanied by a partial opening of the helicase core and leads to the mRNA release with the concomitant displacement of specific mRNA bound proteins (in green) (4). Upon mRNA dissociation, Nup159 is able to bind Dbp5 causing a further opening of both RecA-like domains and the ADP release (5). The recycled enzyme might undergo a new round of its ATPase cycle. Modified from Tieg and Krebber (2013).

During this cycle, the conformation of the enzyme changes in dependency of the nucleotide-state and the bound co-factors Gle1-IP 6 or Nup159 (Figure 13). Nucleotide-free Dbp5 has an open conformation with separated RecA-like domains (Fan et al., 2009). The binding of ATP occurs most likely before its association with mRNPs, because ATP-bound Dbp5 has a higher affinity to RNAs (Figure 13, step 1) (Tran et al., 2007; Weirich et al., 2006). The exact time point of the Gle1-IP 6 recruitment is not clear yet, but these co-factors are needed for the stimulation of the ATPase activity of Dbp5 (Alcazar-Roman et al., 2006; Weirich et al., 2006). Nevertheless, the N-terminal extension still separates the RecA-like domains of ATPbound Dbp5 and prevents its premature ATP-hydrolysis (Collins et al., 2009). Only binding of the single-stranded mRNAs by ATP-Dbp5 removes the $\mathrm{N}$-terminal extension that allows the closure of the helicase core and the complete formation of the catalytic center of the enzyme (step 2). Thus, Dbp5 is able to hydrolyze ATP and the transition into the ADP-bound form is accompanied by a partial opening of both RecA-like domains and alteration of the RNA-binding site (step 3) (Montpetit et al., 
2011). This conformational rearrangement of Dbp5 seems to cause the release of the associated mRNA and the concomitant displacement of specific proteins such as Mex67 and Nab2 from the mRNA (Lund and Guthrie, 2005; Noble et al., 2011; Tran et al., 2007). Upon release of the remodeled mRNP into the cytoplasm, Nup159 is able to bind the now free interaction site in the N-terminal RecA-like domain of Dbp5 (step 4) (Montpetit et al., 2011; Napetschnig et al., 2009; von Moeller et al., 2009). The binding of Nup159 induces a further opening of the helicase core that leads to the ADP displacement and recycling of the enzyme (step 5) (Montpetit et al., 2011; Noble et al., 2011).

In conclusion, Dbp5 is only transiently associated with the mRNA and its different binding partners. A spatial regulation of the ATPase cycle is archived by the alternate interaction of Dbp5 with Nup159 and the Nup42-bound Gle1-IP 6 . Thus, they localize Dbp5 to the cytoplasmic fibrils of the NPCs, where Dbp5 is needed for nuclear mRNA export. Therefore, the ATPase cycle of Dbp5 is perfectly regulated for the remodeling of emerging mRNPs at the cytoplasmic side of the NPC. It remains to be shown, whether one Dbp5 molecule performs multiple rounds of its ATPase cycle on one single mRNP to displace consecutively several proteins. Alternatively, several Dbp5 molecules might act on one mRNP to facilitate its directional transport.

However, it is still unclear how Dbp5 is delivered to this favorable position at the cytoplasmic sides of the NPCs (Tieg and Krebber, 2013). Immunoelectron microscopy studies with Balbiani ring mRNPs of Chironomus tentans salivary gland cells indicate an early recruitment of Dbp5 to the 5'ends of the mRNAs during transcription (Zhao et al., 2002). The authors suggested that Dbp5 remains bound to the mRNAs during nucleoplasmic transfer and translocation through the NPCs, until both arrive at the cytoplasmic fibrils. This model is supported by the observation that Dbp5 accumulates in the nuclei of mRNA export defective mex67-5 cells (Hodge et al., 1999). However, this accumulation does not have to result from Dbp5 molecules tethered to nuclear mRNAs and might also be caused by other effects. This is especially true, as Dbp5 does not accumulate in the nuclei of rat7/nup159 mutants, which exhibit mRNA export defects as well (Hodge et al., 1999). More recently, single mRNP molecule imaging and Dbp5 localization in C. tentans suggested that Dbp5 is primarily recruited from the cytoplasm to the NPCs (Siebrasse et al., 2012). Thus, it is still not clear whether Dbp5 accompanies the mRNPs from the nucleus into the cytoplasm or whether Dbp5 awaits the emerging mRNPs already bound to the cytoplasmic fibrils. It remains possible that free nuclear Dbp5 molecules are actively 
exported by Xpo1 and the Ran GTPase system, because Dbp5 accumulates in the nuclei of mutants of these factors (Hodge et al., 1999) and both mechanism might be used in parallel to deliver Dbp5 to its diverse functions in the cytoplasm.

\subsubsection{Additional functions of Dbp5}

In the cell, Dbp5 is predominantly localized to the cytoplasm with an enrichment at the nuclear envelope (Snay-Hodge et al., 1998; Tseng et al., 1998) adequate for its roles in nuclear mRNA export (section 4.4.2) and translation termination (section 4.4.6). However, Dbp5 does also shuttle between nucleus and cytoplasm, as the protein accumulates in the nucleus of export defective xpo1-1 cells (Hodge et al., 1999). Thus, it is the question, whether Dbp5 has a further function in the nucleus. Estruch and Cole (2003) and Estruch et al. (2012) suggested that Dbp5 is not only co-transcriptionally recruited to the mRNAs, but also acts during transcription initiation, as the RNA-helicase genetically and physically interacts with transcription factors. However, an exact function of Dbp5 in this nuclear process needs to be shown. Finally, different complexes of Dbp5 might exist in the nucleus, the cytoplasm and at the NPCs to realize the diverse functions of the enzyme (Tieg and Krebber, 2013).

\subsubsection{The DEAD-box protein regulator Gle1}

The essential and conserved Gle1 (GLFG lethal) or also named Rss1 (Rat seven suppressor) is a regulator of DEAD-box proteins and functions during nuclear mRNA export and translational processes. In the cell, Gle1 is localized in the cytoplasm and enriched at the nuclear rim, where it interacts with the cytoplasmic fibrils via the nucleoporin Nup42/Rip1 (human hCG1) (Del Priore et al., 1996; Murphy and Wente, 1996; Strahm et al., 1999).

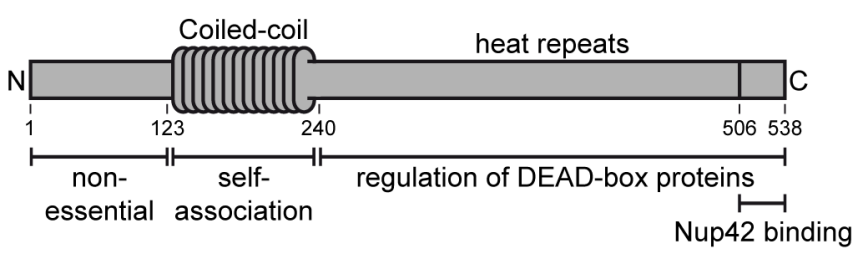

Figure 14: Scheme of the different domains of Gle1 and their functions.

The different domains of Gle1 and their functions are shown. The N-terminal domain is nonessential and has no known function. The middle domain has a predicted coiled-coil structure and is necessary for the self-association of the protein, which is important for mRNA export. The Cterminus regulates DEAD-box proteins and interacts with the nucleoporin Nup42. The numbers represent the amino acids of yeast Gle1. Adapted from Adams et al. (2014). 
Gle1 contains a non-essential N-terminus, a predicted coiled-coil domain and an NES-containing C-terminus, which regulates the DEAD-box proteins and interacts with Nup42 (Figure 14) (Folkmann et al., 2013; Strahm et al., 1999). The essential coiled-coil domain mediates the self-association of Gle1 proteins, which is required for mRNA export (Folkmann et al., 2013). The authors suggested that the dimerization or oligomerization of Gle1 might be necessary to simultaneously interact with Dbp5 and Nup42 during mRNA export, as their binding sites partially overlap (Figure 14). A mutation of the human GLE1 gene that leads to the expression of proteins unable to self-associate causes the autosomal recessive motoneuron disease LCCS1 (lethal congenital contracture syndrome-1) (Folkmann et al., 2013; Nousiainen et al., 2008).

In addition to its function in activation of Dbp5 during nuclear mRNA export and translation termination, Gle1 is also involved in translation initiation (Bolger et al., 2008; Bolger and Wente, 2011). In this process, Gle1 acts independently of Dbp5 and $\mathrm{IP}_{6}$ by inhibition of the ATPase activity of the DEAD-box protein Ded1.

\subsubsection{Dbp5 and Gle1 in translation termination}

Despite their predominant localization in the cytoplasm, the cytoplasmic functions of Dbp5 and Gle1 have been unknown for long time. Finally, Gross et al. (2007) and Bolger et al. (2008) described a role of both proteins in translation termination. The authors revealed that temperature-sensitive mutants of DBP5 and GLE1 are hypersensitive to translational inhibitors and show an increased read-through activity of stop codons in a dual reporter assay indicating defects in the stop codon recognition. Furthermore, Dbp5 and Gle1 are part of polysomal fractions of sucrosedensity gradients and physically and genetically interact with the canonical release factors eRF1 and eRF3. However, their interaction to eRF3 was only RNA-mediated and no protein-protein contact was detected between eRF3 and Dbp5 or Gle1 in vivo. Remarkably, the interaction between eRF1 and eRF3 is decreased in $d b p 5$ and gle1 mutants, which also show a reduced recruitment of eRF3 to the polysomal fractions of sucrose-density gradients. These results led to the model that Dbp5 and Gle1 accompany eRF1 to terminating ribosomes and trigger the subsequent recruitment of eRF3 possibly by remodeling of the ribosomal complex (Figure 15) (Bolger et al., 2008; Gross et al., 2007). Thus, eRF3 would only enter the ribosomal A-site upon release of Dbp5 and Gle1. However, it needs to be shown whether eRF1 and eRF3 are indeed able to separately bind the ribosome or whether recruitment as 
a stable complex is favored. Moreover, the exact functions of Dbp5 and Gle1 during the termination process are not clear yet.
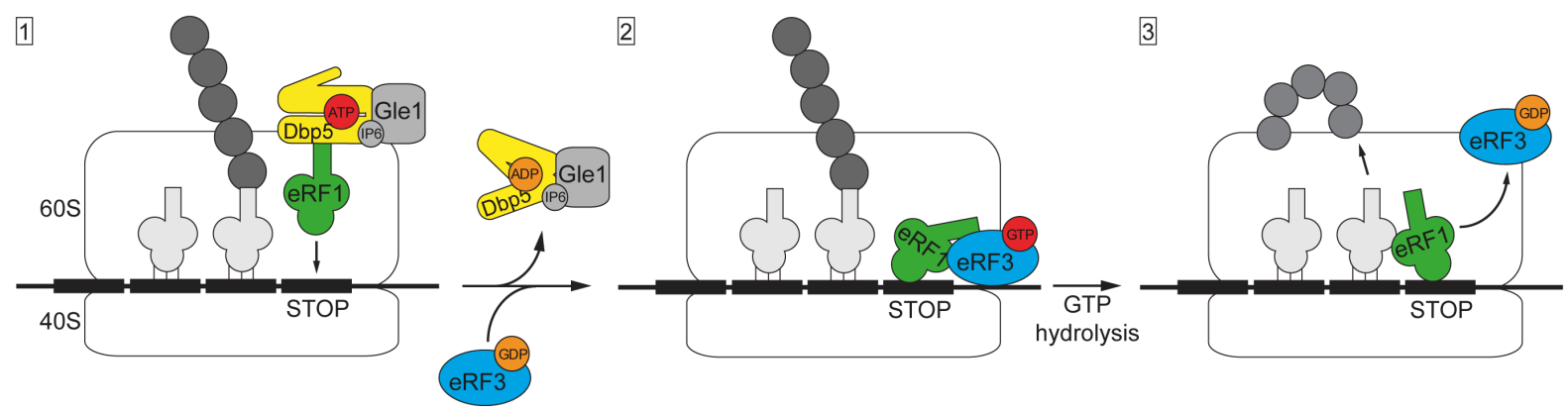

Figure 15: Model of translation termination mediated by Dbp5 and Gle1.

Dbp5 and Gle1-IP 6 might enter together with eRF1 the empty ribosomal A-site of a ribosome that arrived at the stop codon (1). The ATPase activity of Dbp5, which is stimulated by Gle1-IP 6 , might cause the remodeling of the ribosomal complex leading to proper stop codon decoding by eRF1 and possibly to recruitment of eRF3 (2). Upon release of Dbp5 and Gle1-IP 6 , eRF3 can enter the ribosomal A-site and its stimulated GTP-hydrolysis enables the efficient peptide release by eRF1

(3). Adapted from Tieg and Krebber (2013).

Nevertheless, it was shown that the regulated ATPase cycle of Dbp5, known from nuclear mRNA export (Figure 13), is also necessary for efficient translation termination. Firstly, both ATPase activity stimulating cofactors, Gle1 and $\mathrm{IP}_{6}$ are required for translation termination, as gle1 mutants and ipk1 1 cells lacking $\mathrm{IP}_{6}$ exhibit an increased stop codon read-through rate (Alcazar-Roman et al., 2010; Bolger et al., 2008). In particular, gle1 mutants defective for $\mathrm{IP}_{6}$ binding reveal stop codon recognition defects indicating that the interaction of Gle1 and $\mathrm{IP}_{6}$ is needed for efficient termination (Alcazar-Roman et al., 2010). Secondly, overexpression of wild typic DBP5, but not of the ATPase-deficient mutant $d b p 5(E 240 Q)$, rescues the increased stop codon read-through activity of sup45-2 (Gross et al., 2007). Therefore, the ATPase activity of Dbp5, which is stimulated by Gle1 and IP 6 , seems to be necessary for the efficient stop codon recognition by eRF1. Possibly, the ATPase-dependent remodeling activity of Dbp5 might lead to a rearrangement of the ribosomal complex and to the proper positioning of eRF1 in the ribosomal A-site (Baierlein and Krebber, 2010). Thus, it might exist an additional cytoplasmic ADPrelease factor for Dbp5 in translation termination, as Nup159 known from nuclear mRNA export is part of the cytoplasmic fibrils of the NPCs (Tieg and Krebber, 2013). However, definition of the time point, when Dbp5 and Gle1 are recruited to the termination complex and their exact modes of action during translation termination need further investigations. 


\subsubsection{Temperature-sensitive mutants of DBP5}

$D B P 5 / R A T 8$ is an essential gene, so that its deletion is lethal for yeast cells. However, different temperature-sensitive mutants of DBP5 are available and the strains rat8-1, rat8-2, rat8-3 and rat8-7 were used in the present study. All of these dbp5/rat8 alleles contain point mutations, which lead to single amino acid substitutions in the helicase core of Dbp5, as indicated in Figure 16 (Snay-Hodge et al., 1998).

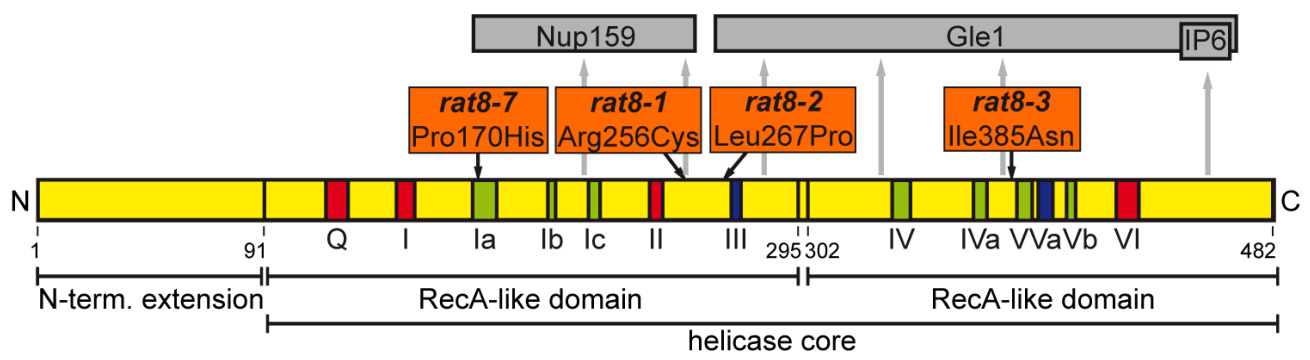

Figure 16: Position of the different temperature-sensitive $d b p 5$ alleles.

The amino acid exchanges resulting from the heat-sensitive rat8-1, rat8-2 and rat8-3 and of the cold-sensitive rat8-7 alleles are labeled in orange and their positions in the helicase core of yeast Dbp5 are indicated.

These cells grow almost like wild type cells at the permissive temperature of $25^{\circ} \mathrm{C}$, while shifts of the heat-sensitive rat8-1, rat8-2 and rat8-3 strains to $37^{\circ} \mathrm{C}$ and of the cold-sensitive rat8- 7 strain to $16^{\circ} \mathrm{C}$ leads to cell death (Snay-Hodge et al., 1998). All of these mutants rapidly accumulate poly(A)-tail containing RNAs in the nucleus upon shift to their restrictive temperatures (Snay-Hodge et al., 1998). Moreover, rat8-2 cells are known to cause an increased rate of stop codon read-through (Gross et al., 2007). However, the impact of these amino acid substitutions on the Dbp5 protein is not structurally and biochemically characterized so far. Snay-Hodge et al. (1998) only revealed that all of these mutated proteins are not thermo-labile and thus, are not degraded at the non-permissive temperatures. Furthermore, for the rat8-2 protein is known that it is localized together with other export factors in cytoplasmic foci, socalled RNA export granules, which might store incomplete remodeled mRNAs (Scarcelli et al., 2008). 


\subsection{Scope of the study}

The best characterized function of the DEAD-box RNA-helicase Dbp5 is its conserved role in nuclear mRNA export, during which it displaces bound transport factors from the emerging mRNAs at the cytoplasmic sides of the NPCs. However, it might be possible that Dbp5 is also involved in the transport of other large ribonucleoprotein complexes such as pre-ribosomal subunits. Indeed, the dbp5 mutants rat8-2 and rat8-1 were listed in a large-scale export screen to accumulate pre-60S subunits in the nucleus, but this effect was not further analyzed (StageZimmermann et al., 2000). Furthermore, pre-40S export defects in dbp5 mutants have not been described so far (Gleizes et al., 2001; Moy and Silver, 1999). Thus, the first part of this study aimed to analyze whether Dbp5 is required for the nuclear export of both pre-ribosomal particles and to characterize its role during this transport process.

Furthermore, Dbp5 and its co-factor Gle1 are involved in efficient translation termination in the cytoplasm (Bolger et al., 2008; Gross et al., 2007) and the ATPbinding cassette protein Rli1 was described as an additional translation termination factor as well (Khoshnevis et al., 2010). However, when and how these proteins are recruited to the termination complex is unclear. Moreover, a comprehensive model of translation termination that includes the canonical release factors eRF1 and eRF3 and all of the novel termination factors is still missing. Therefore, one aim of this study was to investigate Dbp5, Gle1 and Rli1 together during translation termination and to analyze their recruitment to the termination complex. 


\section{MATERIAL and METHODS}

\subsection{Equipment and software}

Table 1: Equipment used in this study.

\begin{tabular}{|c|c|c|}
\hline Machine & Model & Source \\
\hline Cell homogenizer & FastPrep-24 & $\begin{array}{l}\text { MP Biomedicals } \\
\text { (Illkirch/France) }\end{array}$ \\
\hline $\begin{array}{l}\text { Chemiluminescence } \\
\text { detection system }\end{array}$ & Fusion SL 3500.WL & $\begin{array}{l}\text { Peqlab } \\
\text { (Erlangen/Germany) }\end{array}$ \\
\hline \multirow{4}{*}{ Centrifuges } & $\begin{array}{l}\text { Heraeus Pico } 21 \\
\text { Microcentrifuge }\end{array}$ & $\begin{array}{l}\text { Thermo Scientific } \\
\text { (Schwerte/Germany) }\end{array}$ \\
\hline & $\begin{array}{l}\text { Heraeus Fresco } 21 \\
\text { Refrigerated Microcentrifuge }\end{array}$ & $\begin{array}{l}\text { Thermo Scientific } \\
\text { (Schwerte/Germany) }\end{array}$ \\
\hline & $\begin{array}{l}\text { Heraeus Multifuge } \times 3 R \text { with } \\
\text { swinging bucket rotor TX-750 or } \\
\text { Fiberlite F15-8x50cy fixed-angle rotor }\end{array}$ & $\begin{array}{l}\text { Thermo Scientific } \\
\text { (Schwerte/Germany) }\end{array}$ \\
\hline & $\begin{array}{l}\text { Sorvall WX80 ultracentrifuge } \\
\text { with TH-641 rotor }\end{array}$ & $\begin{array}{l}\text { Thermo Scientific } \\
\text { (Schwerte/Germany) }\end{array}$ \\
\hline $\begin{array}{l}\text { Density-gradient } \\
\text { fractionation system }\end{array}$ & $\begin{array}{l}\text { Foxy Jr. Fraction Collector, } \\
\text { Optical Unit Type 11, } \\
\text { Absorbance Detector UA-6 }\end{array}$ & $\begin{array}{l}\text { Teledyne Isco } \\
\text { (Lincoln, Nebraska/USA) }\end{array}$ \\
\hline Electro blotter & $\begin{array}{l}\text { PerfectBlue Semi-Dry Electro blotter } \\
\text { Sedec M }\end{array}$ & $\begin{array}{l}\text { Peqlab } \\
\text { (Erlangen/Germany) }\end{array}$ \\
\hline $\begin{array}{c}\text { Gel documentation } \\
\text { system }\end{array}$ & INTAS UV System & $\begin{array}{l}\text { INTAS GmbH } \\
\text { (Göttingen/Germany) }\end{array}$ \\
\hline Gradient former & Gradient master 108 & $\begin{array}{l}\text { BioComp Instruments } \\
\text { (Fredericton/Canada) }\end{array}$ \\
\hline Hemocytometer & Neubauer improfed & $\begin{array}{l}\text { Carl Roth GmbH } \\
\text { (Karlsruhe/Germany) }\end{array}$ \\
\hline \multirow{3}{*}{ Microscopes } & $\begin{array}{l}\text { Fluorescence microscope } \\
\text { DMI6000B with Leica DFC360 FX camera }\end{array}$ & $\begin{array}{l}\text { Leica Microsystems } \\
\text { (Wetzlar/Germany) }\end{array}$ \\
\hline & $\begin{array}{l}\text { Light microscope } \\
\text { Leitz Biomed Typ 020-507-010 }\end{array}$ & $\begin{array}{l}\text { Leica Microsystems } \\
\text { (Wetzlar/Germany) }\end{array}$ \\
\hline & $\begin{array}{l}\text { Tetrad microscope } \\
\text { Nikon Eclipse E400 }\end{array}$ & $\begin{array}{l}\text { Nikon } \\
\text { (Düsseldorf/Germany) }\end{array}$ \\
\hline Photometer & BioPhotometer & $\begin{array}{l}\text { Eppendorf AG } \\
\text { (Hamburg/Germany) }\end{array}$ \\
\hline Spectrophotometer & NanoDrop2000 & $\begin{array}{l}\text { Thermo Scientific } \\
\text { (Schwerte/Germany) }\end{array}$ \\
\hline Sonifier & Sonifier Cell Disrupter S-250A & $\begin{array}{l}\text { Branson Ultrasonics } \\
\text { (Dietzenbach/Germany) }\end{array}$ \\
\hline Thermocycler & MyCycler Thermocycler & $\begin{array}{l}\text { BioRad } \\
\text { (München/Germany) }\end{array}$ \\
\hline UV-Crosslinker & Bio-Link BLX-E365 & $\begin{array}{l}\text { Vilber Lourmat } \\
\text { (Eberhardzell/Germany) }\end{array}$ \\
\hline $\begin{array}{l}\text { Water purification } \\
\text { system }\end{array}$ & Milli-Q water purification & $\begin{array}{l}\text { Millipore } \\
\text { (Eschborn/Germany) }\end{array}$ \\
\hline X-ray film processor & Optimax & $\begin{array}{l}\text { PROTEC } \\
\text { (Oberstenfeld/Germany) }\end{array}$ \\
\hline
\end{tabular}


Table 2: Software used in this study.

\begin{tabular}{l|l|l}
\hline \multicolumn{1}{c|}{ Application } & \multicolumn{1}{c}{ Software } & \multicolumn{1}{c}{ Source } \\
\hline Picture editing & $\begin{array}{l}\text { Adobe Photoshop CS6; } \\
\text { Adobe Illustrator CS6 }\end{array}$ & $\begin{array}{l}\text { Adobe Systems } \\
\text { (San Jose/USA) }\end{array}$ \\
\hline DNA sequence editor & ApE plasmid editor & $\begin{array}{l}\text { from M. Wayne Davis } \\
\text { (University of Utah/USA) }\end{array}$ \\
\hline $\begin{array}{l}\text { Quantification of fluorescent signals/ } \\
\text { Measurement of peak areas }\end{array}$ & Fiji 1.48s Java 1.6.0_65 & $\begin{array}{l}\text { from W. Rasband } \\
\text { (NIH/USA) }\end{array}$ \\
\hline $\begin{array}{l}\text { Quantification of chemi- } \\
\text { luminescent signals }\end{array}$ & Bio-1D & $\begin{array}{l}\text { Peqlab } \\
\text { (Erlangen/Germany) }\end{array}$ \\
\hline $\begin{array}{l}\text { Statistical analyses } \\
\text { Text processing }\end{array}$ & Microsoft Excel 2010 & $\begin{array}{l}\text { Microsoft Corporation } \\
\text { (Redmond/USA) }\end{array}$ \\
\hline Microscopy pictures & Microsoft Word 2010 & $\begin{array}{l}\text { Microsoft Corporation } \\
\text { (Redmond/USA) }\end{array}$ \\
\hline
\end{tabular}

\subsection{Chemicals and consumable material}

Table 3: Particular materials and chemicals used in this study.

\begin{tabular}{|c|c|}
\hline Materials/Chemicals & Source \\
\hline Agarose NEEO Ultra-Quality & Carl Roth (Karlsruhe/Germany) \\
\hline Amersham Hybond- $\mathrm{N}^{+}$membrane & GE Healthcare (Freiburg/Germany) \\
\hline Amersham Protran $0.45 \mu \mathrm{m}$ nitrocellulose membrane & GE Healthcare (Freiburg/Germany) \\
\hline Blocking reagent & Roche (Mannheim/Germany) \\
\hline Complete, EDTA-free protease inhibitor & Roche (Mannheim/Germany) \\
\hline CSPD & Roche (Mannheim/Germany) \\
\hline Cycloheximide & Carl Roth (Karlsruhe/Germany) \\
\hline Deionized Formamide & AppliChem (München/Germany) \\
\hline dNTPs & Thermo Scientific (Schwerte/Germany) \\
\hline 5-Fluoroorotic acid & Apollo Scientific (Derbyshire/UK) \\
\hline Formaldehyde $37 \%$ & AppliChem (München/Germany) \\
\hline GFP-Trap_A & ChromoTek (Planegg-Martinsried/Germany) \\
\hline Glass beads type S 0.4-0.6 mm & Carl Roth (Karlsruhe/Germany) \\
\hline Glutathione Sepharose 4B beads & GE Healthcare (Freiburg/Germany) \\
\hline IgG Sepharose 6 FastFlow & GE Healthcare (Freiburg/Germany) \\
\hline IPTG & Carl Roth (Karlsruhe/Germany) \\
\hline Microscope slides, 12 wells, $5.2 \mathrm{~mm}$, PTFE-coating & Thermo Scientific (Schwerte/Germany) \\
\hline Phenol/chloroform/isoamyl alcohol (25:24:1) & Carl Roth (Karlsruhe/Germany) \\
\hline Poly-L-lysine hydrobromide & Sigma-Aldrich (München/Germany) \\
\hline Protino Ni-IDA Resin & Macherey-Nagel (Düren/Germany) \\
\hline Protease inhibitor cocktail for yeast & Sigma-Aldrich (München/Germany) \\
\hline Salmon Sperm-carrier DNA & Sigma-Aldrich (München/Germany) \\
\hline Rotiphorese Gel 30 (37.5:1) & Carl Roth (Karlsruhe/Germany) \\
\hline tRNAs & Sigma-Aldrich (München/Germany) \\
\hline Whatman Blotting Paper $0.8 \mathrm{~mm}$ & Hahnemühle FineArt (Dassel/Germany) \\
\hline
\end{tabular}


Table 4: Kits used in this study.

\begin{tabular}{l|l}
\hline \multicolumn{1}{c|}{ Kits } & \multicolumn{1}{c}{ Source } \\
\hline Amersham ECL Prime Western Blotting Detection Kit & GE Healthcare (Freiburg/Germany) \\
\hline DIG RNA labeling mix, 10x conc. & Roche (Mannheim/Germany) \\
\hline NucleoBond PC 100 & Macherey-Nagel (Düren/Germany) \\
\hline NucleoSpin Plasmid & Macherey-Nagel (Düren/Germany) \\
\hline NucleoSpin RNA & Macherey-Nagel (Düren/Germany) \\
\hline peqGOLD Gel extraction Kit & Peqlab (Erlangen/Germany) \\
\hline
\end{tabular}

Table 5: Size standards used in this study.

\begin{tabular}{l|c|c}
\hline \multicolumn{1}{c|}{ Size standard } & Application & Source \\
\hline Lambda DNA/EcoRI+HindIII Marker & Agarose gels & Thermo Scientific (Schwerte/Germany) \\
\hline PageRuler Prestained Protein Ladder & SDS-PAGE & Thermo Scientific (Schwerte/Germany) \\
\hline PageRuler Unstained Protein Ladder & SDS-PAGE & Thermo Scientific (Schwerte/Germany) \\
\hline
\end{tabular}

All other chemicals, solutions and consumable materials used in this study were purchased from the following companies unless otherwise stated:

Apollo Scientific Limited (Derbyshire/UK), AppliChem (München/Germany), B. Braun (Melsungen/Germany), BD Biosciences (Heidelberg/Germany), Carl Roth (Karlsruhe/Germany), Difco (Detroit/USA), Fisher Scientific (Nidderau/Germany), GE Healthcare (Freiburg/Germany), Invitrogen (Frankfurt am Main/Germany), Merck (Darmstadt/Germany), New England Biolabs (Frankfurt am Main/Germany), OMNILAB GmbH (Bremen/Germany), Promega (Mannheim/Germany), Roche Diagnostics (Mannheim/Germany), Sarstedt (Nürnbrecht/Germany), Serva Feinbiochemika (Heidelberg/Germany), Sigma-Aldrich (München/Germany), Thermo Scientific (Schwerte/Germany), Th. Geyer (Renningen/Germany) and VWR International (Darmstadt/Germany).

\subsection{Enzymes and Antibodies}

All enzymes listed in Table 6 were used with their appropriate buffer and according to the protocols of the manufacturers.

Table 6: Enzymes used in this study.

\begin{tabular}{l|l}
\hline \multicolumn{1}{c|}{ Enzyme } & \multicolumn{1}{c}{ Source } \\
\hline DreamTaq DNA polymerase & Thermo Scientific (Schwerte/Germany) \\
\hline FastAP Thermosensitive Alkaline Phosphatase & Thermo Scientific (Schwerte/Germany) \\
\hline Phusion High-Fidelity DNA polymerase & New England Biolabs (Frankfurt/Germany) \\
\hline Restriction enzymes & Thermo Scientific (Schwerte/Germany) \\
\hline Restriction enzymes & New England Biolabs (Frankfurt/Germany) \\
\hline RiboLock RNase inhibitor & Thermo Scientific (Schwerte/Germany)
\end{tabular}




\begin{tabular}{l|l}
\multicolumn{1}{c|}{ Enzyme } & \multicolumn{1}{c}{ Source } \\
\hline RNase A & AppliChem (München/Germany) \\
\hline T4-DNA-ligase & Thermo Scientific (Schwerte/Germany) \\
\hline T5 Exonuclease & New England Biolabs (Frankfurt/Germany) \\
\hline T7-RNA-polymerase & Thermo Scientific (Schwerte/Germany) \\
\hline Taq DNA ligase & New England Biolabs (Frankfurt/Germany) \\
\hline Velocity DNA polymerase & Bioline (Luckenwalde/Germany) \\
\hline Zymolyase 20T & Seikagaku corporation (Tokyo/Japan) \\
\hline
\end{tabular}

All antibodies that were listed in Table 7 and used for detection in Western blot analyses were diluted in $2 \%(\mathrm{w} / \mathrm{v})$ milk powder / TBST. The dilutions were stored at $-20^{\circ} \mathrm{C}$ for multiple usages.

Table 7: Antibodies used in this study. The dilutions for usage in Western blotting (WB), Northern blotting (NB) or Fluorescence in situ hybridizations (FISH) are indicated.

\begin{tabular}{l|l|l}
\hline \multicolumn{1}{c|}{ Antibody (Organism) } & \multicolumn{1}{c}{ Dilution (WB) } & \multicolumn{1}{c}{ Source } \\
\hline Anti-Aco1 (rabbit) & $1: 2000$ & gift from R. Lill (Marburg/Germany) \\
\hline $\begin{array}{l}\text { Anti-Dbp5 (rabbit) } \\
\text { (against full length Dbp5) }\end{array}$ & $1: 1000$ & $\begin{array}{l}\text { Peptide Specialty Laboratories } \\
\text { (Heidelberg/Germany) }\end{array}$ \\
\hline $\begin{array}{l}\text { Anti-Digoxigenin-AP, } \\
\text { Fab fragments (sheep) }\end{array}$ & $1: 10000$ (NB) & Roche (Mannheim/Germany) \\
\hline Anti-Digoxigenin Fab-FITC (sheep) & $1: 200$ (FISH) & Roche (Mannheim/Germany) \\
\hline Anti-Fech (rabbit) & $1: 7000$ & gift from R. Lill (Marburg/Germany) \\
\hline Anti-GFP (FL) (rabbit) & $1: 1000$ & Santa Cruz (Heidelberg/Germany) \\
\hline Anti-GST (B-14) (mouse) & $1: 2000$ & Santa Cruz (Heidelberg/Germany) \\
\hline Anti-HA (12CA5) (mouse) & $1: 750$ & Santa Cruz (Heidelberg/Germany) \\
\hline Anti-Mex67 (rabbit) & $1: 50000$ & gift from C. Dargemont (Paris/France) \\
\hline Anti-Myc (A-14) (rabbit) & $1: 750$ & Santa Cruz (Heidelberg/Germany) \\
\hline Anti-Por1 (rabbit) & $1: 2000$ & gift from R. Lill (Marburg/Germany) \\
\hline Anti-Rpl35 (rabbit) & $1: 5000$ & gift from M. Seedorf (Heidelberg/Germany) \\
\hline Anti-Rps3 (rabbit) & $1: 10000$ & gift from M. Seedorf (Heidelberg/Germany) \\
\hline $\begin{array}{l}\text { Anti-Rps3 (rabbit) } \\
\text { (peptide antibody) }\end{array}$ & $1: 1000$ & $\begin{array}{l}\text { Davids Biotechnologie } \\
\text { (Regensburg/Germany) }\end{array}$ \\
\hline Anti-Sup45 (rabbit) & $1: 1000$ & gift from D. Bedwell (Birmingham/USA) \\
\hline Anti-Zwf1 (rabbit) & $1: 4000$ & gift from R. Lill (Marburg/Germany) \\
\hline Anti-rabbit IgG (H+L)-HRPO & $1: 15000$ - & Dianova (Hamburg/Germany) \\
\hline Anti-mouse IgG (H+L)-HRPO & $1: 25000$ & Dianova (Hamburg/Germany) \\
\hline
\end{tabular}

\subsection{Oligonucleotides}

All oligonucleotides listed in Table 8 were purchased from Sigma-Aldrich (München/ Germany), diluted in nuclease-free, deionized water to $10 \mu \mathrm{M}$ and stored at $-20^{\circ} \mathrm{C}$. 
Table 8: Oligonucleotides used in this study.

\begin{tabular}{|c|c|c|c|}
\hline $\begin{array}{c}\text { Oligo } \\
\text { number }\end{array}$ & Sequence & Target & Features \\
\hline HK974 & $\begin{array}{l}\text { 5'-taatacgactcactataggg } \\
\text { GCTTAAGTTCAGCGGGTACTCCTACC-3' }\end{array}$ & RDN25 reverse & T7 promoter \\
\hline HK1138 & 5'-AGGTAGGAGTACCCGCTGAA-3' & RDN25 forward & \\
\hline HK1139 & $\begin{array}{l}\text { 5'-taatacgactcactataggg } \\
\text { ATGGAATTTACCACCCACTTAGAGC-3' }\end{array}$ & $R D N 25$ reverse & T7 promoter \\
\hline HK1140 & 5'-GTGAAACTGCGAATGGCTCATTAAAT-3' & RDN18 forward & \\
\hline HK1141 & $\begin{array}{l}\text { 5'-taatacgactcactataggg } \\
\text { AATCGAACCCTTATTCCCCGTTA-3' }\end{array}$ & $R D N 18$ reverse & T7 promoter \\
\hline HK1485 & 5'-tatCCGCGGTGCCCAAGTCAAGCCTAC-3' & $\begin{array}{l}\text { RPL11B promoter } \\
\text { forward }\end{array}$ & Sacll site \\
\hline HK1486 & 5'-ctaCTCGAGcTTTATCGAGCACATCAGCG-3' & $R P L 11 B$ reverse & Xhol site \\
\hline HK1623 & 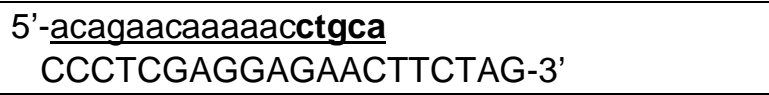 & $\begin{array}{l}\text { LEU2 promoter } \\
\text { forward }\end{array}$ & $\begin{array}{c}\text { Pstl site } \\
\text { homolog to URA3 }\end{array}$ \\
\hline HK1624 & 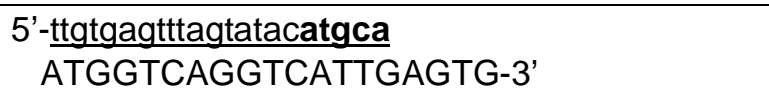 & $\begin{array}{l}\text { LEU2 3'UTR } \\
\text { reverse }\end{array}$ & $\begin{array}{c}\text { Nsil site } \\
\text { homolog to URA3 }\end{array}$ \\
\hline HK1723 & 5'-CGAGCCGTTTATGTCCAACG-3' & LSR1 forward & \\
\hline HK1724 & $\begin{array}{l}\text { 5'-taataggactcactatagg } \\
\text { GCCGATACTTGGGGGATAAG-3' }\end{array}$ & LSR1 reverse & T7 promoter \\
\hline HK1893 & 5'-GTAGGTGAACCTGCGGAAGG-3' & RDN18 forward & \\
\hline HK1894 & $\begin{array}{l}\text { 5'-taatacgactcactataggg } \\
\text { GCACAGAAATCTCTCACCGTTTG-3' }\end{array}$ & ITS1 reverse & T7 promoter \\
\hline HK1895 & 5'-GCCTGTTTGAGCGTCATTTCCTTCTC-3' & $\begin{array}{l}\text { RDN5.8/ITS2 } \\
\text { forward }\end{array}$ & \\
\hline
\end{tabular}

\subsection{Strains}

\subsubsection{Saccharomyces cerevisiae strains}

Table 9: Saccharomyces cerevisiae strains used in this study.

\begin{tabular}{|c|c|c|}
\hline $\begin{array}{c}\text { Strain } \\
\text { number }\end{array}$ & Genotype & Source \\
\hline HKY36 & MAT $\alpha$ ura3-52 leu2 $\Delta 1$ his3 $\Delta 200$ & (Winston et al., 1995) \\
\hline HKY40 & $\begin{array}{l}\text { MAT? ura3-1 leu2-3 trp1-1 his3-11 ade2-1 xpo1::LEU2 } \\
+ \text { pCEN XPO1 HIS3 }\end{array}$ & (Stade et al., 1997) \\
\hline HKY41 & $\begin{array}{l}\text { MAT } \alpha \text { ura3-1 leu2-3 trp1-1 his3-11 ade2-1 can1-1 } \\
\text { xpo1::LEU2 + pCEN xpo1-1 HIS3 }\end{array}$ & (Stade et al., 1997) \\
\hline HKY90 & MATa ura2 leu2 rat8-1 & Laboratory of H. Krebber \\
\hline HKY93 & MAT $\alpha$ ura3-1 leu2-3,112 his3-11 ade2-1 gle1-4 & (Murphy and Wente, 1996) \\
\hline HKY124 & MAT $\alpha$ ura3-52 leu2 $\Delta 1$ his $3 \Delta 200$ rat7-1 & (Del Priore et al., 1997) \\
\hline HKY128 & $\begin{array}{l}\text { MAT } \alpha \text { ura3-52 leu2 } 21 \text { trp } 1 \Delta 63 \text { his } 3 \Delta 200 \text { rat8::HIS3 } \\
+ \text { pCEN rat8-3 LEU2 }\end{array}$ & (Snay-Hodge et al., 1998) \\
\hline HKY129 & $\begin{array}{l}\text { MATa ura3-52 leu2 } 21 \text { trp1 } 163 \text { his } 3 \Delta 200 \text { rat8::HIS3 } \\
+ \text { pCEN rat8-7 LEU2 }\end{array}$ & (Snay-Hodge et al., 1998) \\
\hline HKY130 & MATa ura3-52 leu2 $\Delta 1$ trp1 $\Delta 63$ rat8-2 & (Snay-Hodge et al., 1998) \\
\hline HKY276 & MATa ade2 his3 leu2 trp1 ura3 & (Zenklusen et al., 2001) \\
\hline HKY314 & MATa ura3 $\Delta 0$ leu2 $\Delta 0$ his $3 \Delta 1$ met $15 \Delta 0$ & Euroscarf \\
\hline
\end{tabular}




\begin{tabular}{|c|c|c|}
\hline $\begin{array}{l}\text { Strain } \\
\text { number }\end{array}$ & Genotype & Source \\
\hline HKY445 & MAT $\alpha$ ura3-1 his5-2 can1-100 & (Stansfield et al., 1997) \\
\hline HKY446 & MAT $\alpha$ ura3-1 ade2-1 his5-2 can1-100 sup45-2 & (Stansfield et al., 1997) \\
\hline HKY456 & $\begin{array}{l}\text { MATa ura3-52 leu2 } 21 \text { trp1 } 163 \text { his } 3 \Delta 200 \text { rat8::HIS3 } \\
+p 2 \mu \text { RAT8 URA3 }\end{array}$ & Laboratory of $\mathrm{H}$. Krebber \\
\hline HKY473 & MATa ura2 leu2 ade2 trp1 lys2 sup45-2 & (Gross et al., 2007) \\
\hline HKY474 & MATa ura2 leu2 trp1 & Laboratory of $\mathrm{H}$. Krebber \\
\hline HKY617 & $\begin{array}{l}\text { MATa ade2 arg4 leu2-3,112 trp1-289 ura3-52 } \\
\text { DBP5:TAP-K.I.URA3 }\end{array}$ & Euroscarf \\
\hline HKY618 & MATa ade2 arg4 leu2-3,112 trp1-289 ura3-52 & Euroscarf \\
\hline HKY734 & 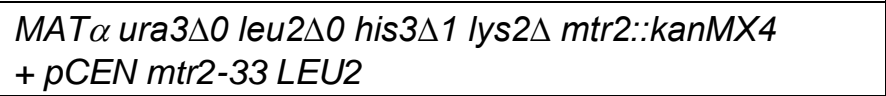 & Laboratory of $\mathrm{H}$. Krebber \\
\hline HKY863 & $\begin{array}{l}\text { MAT } \alpha \text { ura3 } \Delta 0 \text { leu2 } \Delta 0 \text { his3 } \Delta 1 \text { lys } 2 \Delta \text { rp/10::kanMX4 } \\
+ \text { pCEN rpl10(G161D)-GFP URA3 }\end{array}$ & (Baierlein et al., 2013) \\
\hline HKY892 & $\begin{array}{l}\text { MATa ura3 leu2 trp1 his3 ade2 mtr2::HIS3 } \\
+ \text { pCEN mtr2-33 TRP1 + pCEN MTR2 URA3 }\end{array}$ & (Bassler et al., 2001) \\
\hline HKY894 & $\begin{array}{l}\text { MAT } \alpha \text { ura3 leu2 trp1 his3 lys2 nmd3::kanMX4 } \\
+ \text { pCEN nmd3-2 TRP1 + pCEN NMD3 URA3 }\end{array}$ & (Bassler et al., 2001) \\
\hline HKY1070 & MATa ade2-1 ura3-1 HIS3 trp1-1 leu2-3,112 can1-100 & (Bolger et al., 2008) \\
\hline HKY1242 & $\begin{array}{l}\text { MATa ura3 } \Delta 0 \text { leu2 } \Delta 0 \text { his } 3 \Delta 1 \text { met } 15 \Delta 0 \\
\text { RAT8-GFP:HIS3MX6X }\end{array}$ & Invitrogen \\
\hline HKY1356 & $\begin{array}{l}\text { MATa ura3 leu2 trp1 his3 nmd3::kanMX4 rat8::HIS3 } \\
+p 2 \mu \text { RAT8 URA3 + pCEN nmd3-2 TRP1 } \\
+p \text { CEN NMD3 URA3 }\end{array}$ & this study \\
\hline HKY1369 & $\begin{array}{l}\text { MAT } \alpha \text { ura3 leu2 trp1 his3 lys } 2 \text { mtr2::kanMX4 rat8::HIS3 } \\
+ \text { pCEN rat8-3 LEU2 + pCEN MTR2 URA3 }\end{array}$ & this study \\
\hline HKY1370 & MATa ura3 leu2 his3 & this study \\
\hline HKY1371 & $\begin{array}{l}\text { MATa ura3 leu2 trp1 his3 lys2 rat8::HIS3 } \\
+ \text { pCEN rat8-3 LEU2 }\end{array}$ & this study \\
\hline HKY1372 & $\begin{array}{l}\text { MAT } \alpha \text { ura3 leu2 his3 mtr2::kanMX4 } \\
+ \text { pCEN mtr2-33 LEU2 + pCEN MTR2 URA3 }\end{array}$ & this study \\
\hline HKY1493 & MATa ade2 ura3-0 his3-1 leu2-0 trp1 drg1-18 & (Zakalskiy et al., 2002) \\
\hline
\end{tabular}

\subsubsection{Escherichia coli strains}

Table 10: Escherichia coli strains used in this study.

\begin{tabular}{|c|c|c|}
\hline Strain & Genotype & Application \\
\hline $\mathrm{DH} 5 \alpha$ & $\begin{array}{l}\text { F- Ф80lacZAM15 } \Delta \text { (lacZYA-argF) U169 recA1 endA1 } \\
\text { hsdR17 (rK-, mK+) phoA supE44 } \lambda-\text { thi-1 gyrA96 relA1 }\end{array}$ & $\begin{array}{l}\text { Cloning and amplification } \\
\text { of plasmid-DNA }\end{array}$ \\
\hline $\begin{array}{l}\text { Rosetta } 2 \\
\text { (DE3) }\end{array}$ & $\begin{array}{l}\mathrm{F}^{-} \text {omp } \mathrm{T} \text { gal dcm lon } h s d \mathrm{~S}_{\mathrm{B}}\left(r_{B^{-}}, m_{B^{-}}\right) \lambda(\mathrm{DE} 3) \\
\text { pRARE2 }\left(\mathrm{Cam}^{\mathrm{R}}\right)\end{array}$ & $\begin{array}{l}\text { expression of } \\
\text { recombinant proteins }\end{array}$ \\
\hline
\end{tabular}

\subsection{Plasmids}

The concentration of the plasmids listed in Table 11 was adjusted to $1 \mu \mathrm{g} / \mu \mathrm{l}$ with deionized, nuclease-free water and all plasmids were stored at $-20^{\circ} \mathrm{C}$. 
Table 11: Plasmids used in this study.

\begin{tabular}{|c|c|c|}
\hline $\begin{array}{l}\text { Plasmid } \\
\text { number }\end{array}$ & Features & Source \\
\hline pHK20 & CEN MEX67-GFP LEU2 & (Santos-Rosa et al., 1998) \\
\hline pHK43 & CEN XPO1-GFP URA3 & (Stade et al., 1997) \\
\hline pHK86 & CEN TRP1 & (Sikorski and Hieter, 1989) \\
\hline pHK87 & CEN LEU2 & (Sikorski and Hieter, 1989) \\
\hline pHK88 & CEN URA3 & (Sikorski and Hieter, 1989) \\
\hline pHK258 & $2 \mu P_{G A L 1} C B P 80-G F P$ URA3 & (Shen et al., 2000) \\
\hline pHK284 & CEN MTR2 URA3 & (Santos-Rosa et al., 1998) \\
\hline pHK636 & $2 \mu P_{G A L} M Y C-D B P 5$ LEU2 & Laboratory of H. Krebber \\
\hline pHK638 & CEN rat8-3 LEU2 & (Snay-Hodge et al., 1998) \\
\hline pHK653 & CEN GFP-DBP5 URA3 & (Gross et al., 2007) \\
\hline pHK655 & CEN GFP-rat8-2 LEU2 & (Gross et al., 2007) \\
\hline pHK666 & CEN GFP-DBP5 LEU2 & Laboratory of H. Krebber \\
\hline pHK697 & CEN RPS2-GFP URA3 & (Milkereit et al., 2003) \\
\hline pHK698 & CEN RPL25-GFP URA3 & (Gadal et al., 2001) \\
\hline pHK706 & CEN mtr2-33 TRP1 & (Bassler et al., 2001) \\
\hline pHK707 & CEN mtr2-33 LEU2 & (Bassler et al., 2001) \\
\hline pHK720 & CEN NMD3-13xmyc LEU2 & (Ho et al., 2000) \\
\hline pHK724 & CEN NMD3-GFP URA3 & (Hedges et al., 2005) \\
\hline pHK887 & CEN (?) $P_{R L I 1} R L I 1-H A$ LEU2 & $\begin{array}{c}\text { gift from R. Lill } \\
\text { (Marburg/Germany) }\end{array}$ \\
\hline pHK1288 & pGEX-6P-1 (expression plasmid for GST) & GE Healthcare \\
\hline pHK1289 & pGEX-6P-1-DBP5 (expression plasmid for GST-Dbp5) & Laboratory of H. Krebber \\
\hline pHK1292 & CEN (?) $P_{R L I 1} R L I 1-G F P$ LEU2 & Laboratory of H. Krebber \\
\hline pHK1323 & CEN $P_{A D H 1} 3 \times M Y C-G L E 1$ URA3 & Laboratory of H. Krebber \\
\hline pHK1349 & CEN RPL11B-GFP URA3 & this study \\
\hline pHK1372 & $\begin{array}{l}\text { pProEX-1-MTR2-RBS-MEX67 } \\
\text { (expression plasmid for His-Mtr2 and Mex67) }\end{array}$ & (Yao et al., 2007) \\
\hline pHK1380 & CEN P $P_{A D H 1} 3 \times M Y C-G L E 1$ LEU2 & this study \\
\hline pHK1398 & $2 \mu P_{G A L} d b p 5-R 369 G$ LEU2 & (Hodge et al., 2011) \\
\hline pHK1399 & $2 \mu P_{G A L} d b p 5-R 426 Q$ LEU2 & (Hodge et al., 2011) \\
\hline
\end{tabular}

\subsection{Cell cultivation}

Before usage, media were generally autoclaved and heat-sensitive components such as antibiotics or galactose were sterile-filtered and added after autoclaving. To produce solid agar plates, the corresponding liquid medium was supplemented for E. coli with $1.5 \%(\mathrm{w} / \mathrm{v})$ or for yeast with $1.8 \%(\mathrm{w} / \mathrm{v})$ agar.

\subsubsection{Cultivation of E. coli cells}

Cultivation of bacteria cells was performed according to standard protocols (Sambrook et al., 1989). 
LB medium (low salt) (pH 7.5): $\quad 1.0 \%(\mathrm{w} / \mathrm{v})$ Peptone

$0.5 \%(w / v)$ Yeast extract

$0.5 \%(\mathrm{w} / \mathrm{v}) \mathrm{NaCl}$

2xYT medium (pH 7.0): $\quad 1.6 \%(\mathrm{w} / \mathrm{v})$ Peptone

$1.0 \%(\mathrm{w} / \mathrm{v})$ Yeast extract

$0.5 \%(\mathrm{w} / \mathrm{v}) \mathrm{NaCl}$

2xYT medium was used for cultivation of E. coli Rosetta 2 cells for the expression and purification of recombinant proteins (see section 5.12.3). Apart from that, LB medium was standardly used for cultivation of $E$. coli cells. To select for plasmids with specific resistance genes, the media were supplemented with the corresponding antibiotics: $100 \mu \mathrm{g} / \mathrm{ml}$ of Ampicillin, $20 \mu \mathrm{g} / \mathrm{ml}$ of Kanamycin or $34 \mu \mathrm{g} / \mathrm{ml}$ of Chloramphenicol, respectively. To cultivate bacteria cells, liquid cultures were inoculated with single colonies from agar plates and incubated with agitation at $\sim 160 \mathrm{rpm}$ and $37^{\circ} \mathrm{C}$ overnight. The optical density of the cells at $600 \mathrm{~nm}$ was measured by using a photometer.

\subsubsection{Cultivation of S. cerevisiae cells}

Yeast cells were cultivated according to standard protocols (Rose et al., 1990; Sherman, 1991).

YPD medium: $\quad 1 \%(w / v)$ Yeast extract

$2 \%(w / v)$ Peptone

$2 \%(w / v)$ Glucose

Selective medium: $\quad 0.2 \%(\mathrm{w} / \mathrm{v})$ Yeast dropout mix

$0.17 \%(w / v)$ Yeast nitrogen base w/o amino acids \& ammonium sulfate

$0.51 \%(w / v)$ Ammonium sulfate

$2 \%(w / v)$ Glucose

The selective medium was chosen according to the plasmid- or genome-encoded selection marker gene, which complements a metabolic auxotrophy of the yeast strain. Thus, the product of this metabolic pathway was missing in the selective medium. All other needed amino acids and nucleobases were ingredients of the yeast dropout mix, which was composed according to the formula of Sherman (1991). 
FOA plates: $0.2 \%(\mathrm{w} / \mathrm{v})$ Yeast dropout mix (including uracil)

$0.17 \%(\mathrm{w} / \mathrm{v})$ Yeast nitrogen base w/o amino acids \& ammonium sulfate

$0.51 \%(w / v)$ Ammonium sulfate

$0.1 \%(w / v)$ 5-Fluoroorotic acid (FOA)

$2 \%(w / v)$ Glucose

$1.8 \%(w / v)$ Agar

The liquid medium was sterile-filtered and added to the agar upon autoclaving. FOAplates were used to select for uracil auxotroph cells, as the product of the URA3 gene generates the toxic 5-Fluorouracil from 5-Fluoroorotic acid.

Yeast cells were standardly cultivated at $25^{\circ} \mathrm{C}$, as temperature-sensitive strains were used. For that, liquid cultures with appropriate media were inoculated with overnight cultures or single colonies from agar plates and incubated with agitation at $25^{\circ} \mathrm{C}$, until log-phase $\left(1-3 \times 10^{7}\right.$ cells $\left./ \mathrm{ml}\right)$ was achieved. Cell numbers were determined by counting with a hemocytometer. If required, temperatures-sensitive yeast strains were shifted to their non-permissive temperatures in a water bath. Subsequently, large volumes (>200 ml) of log-phase yeast cells were harvested by centrifugation for $10 \mathrm{~min}$ at $4100 \times \mathrm{g}$ and $4^{\circ} \mathrm{C}$ in large $500 \mathrm{ml}$-centrifuge tubes, while small volumes were pelleted by centrifugation for $5 \mathrm{~min}$ at $2050 \times \mathrm{g}$ and $4^{\circ} \mathrm{C}$ in $50 \mathrm{ml}$-falcons. The resulting cell pellets were freshly used or frozen in liquid nitrogen and stored at $-20^{\circ} \mathrm{C}$ for following experiments.

For the expression of galactose-inducible promoters, cells were grown to log-phase in $2 \%(\mathrm{w} / \mathrm{v})$ raffinose-containing media, subsequently induced by the addition of $2 \%(\mathrm{w} / \mathrm{v})$ galactose and further incubated for the indicated time periods.

To perform growth analyses, yeast cells were spotted in 10 fold serial dilutions (from $10^{5}$ to 10 cells / per drop) onto selective or FOA-containing agar plates and were incubated for two to three days at the indicated temperatures.

Glycerol-stocks were produced for the long-time storage of yeast cells. For that, the desired strain was plated onto agar plates and the grown cells were resuspended in $800 \mu \mathrm{l}$ of $50 \%(\mathrm{v} / \mathrm{v})$ glycerol and stored at $-80^{\circ} \mathrm{C}$.

\subsection{Generation of yeast strains}

\subsubsection{Sporulation, tetrad dissection and tetrad analyses}

Mating, sporulation and tetrad dissection was performed, as basically described by Sherman (1991) and Sherman and Hicks (1991). 
Super-SPO medium: $\quad$ Solution 1: $\quad 0.5 \%(w / v)$ Yeast extract

(Rose et al., 1990)

306 mM Potassium acetate

$\begin{array}{ll}\text { Solution 2: } & 5 \mathrm{mM} \text { Glucose } \\ & 0.4 \mathrm{mM} \text { Adenine } \\ & 0.7 \mathrm{mM} \text { Uracil } \\ & 0.4 \mathrm{mM} \text { Tyrosine } \\ & 0.2 \mathrm{mM} \text { Histidine } \\ & 0.3 \mathrm{mM} \text { Leucine } \\ & 0.2 \mathrm{mM} \text { Lysine } \\ & 0.2 \mathrm{mM} \text { Tryptophan } \\ & 0.3 \mathrm{mM} \text { Methionine } \\ & 0.2 \mathrm{mM} \text { Arginine } \\ & 1.2 \mathrm{mM} \text { Phenylalanine } \\ 5.9 \mathrm{mM} \text { Threonine }\end{array}$

First, solution 1 was autoclaved and solution 2 was sterile-filtered and subsequently, both solutions were mixed 1:1.

To generate diploid zygotes, two haploid strains with different mating types were combined on YPD-plates, incubated at $25^{\circ} \mathrm{C}$ and subsequently plated onto appropriate agar plates that select for diploid cells. The growing diploid cells were transferred into $2 \mathrm{ml}$ of Super-SPO medium and incubated for three to seven days with agitation at $25^{\circ} \mathrm{C}$ to induce sporulation by nutrient deficiency. Upon appearance of tetrads, the tetrad dissection was performed. For that, $100 \mu \mathrm{l}$ of the culture were incubated with $40 \mu \mathrm{g}$ zymolyase, until digestion of the ascus wall was visible with the light microscope. Then, washing with P-solution stopped the reaction and subsequently, the cells were transferred onto YPD-plates. By using a tetrad microscope with a micromanipulator, the four spores of one tetrad were separated on the agar plate, which was subsequently incubated for two to five days at $25^{\circ} \mathrm{C}$. The genotypes of the germing spores were analyzed by plating them onto different selective agar plates or by extraction of their chromosomal DNA and performing analytic PCRs.

\subsubsection{Mating type determination}

The mating assay was performed according to Sprague (1991).

B-plates: $\quad 0.17 \%(\mathrm{w} / \mathrm{v})$ Yeast nitrogen base w/o amino acids \& ammonium sulfate $0.51 \%(w / v)$ Ammonium sulfate

$0.3 \%(w / v)$ Agar

$2 \%(w / v)$ Glucose 
To determine the mating type (MATa or MATa) of haploid yeast strains, these cells were replica-plated onto two agar plates that were covered with a lawn of defined MATa- or MATa tester strains, respectively. Upon three days incubation, the grown cells were plated onto B-plates, which select for diploid cells that mated before. The tester strains were isoleucine and valine auxotroph, while the haploid strains with the unknown mating type carried other auxotrophic markers. Thus, only cells with opposite mating type were able to form diploid zygotes, which complement the nutritional defects and allow growth on the selective B-plates.

\subsubsection{Generation of yeast strains HKY1356 and HKY1369-1372}

The S. cerevisiae strain HKY1356 was generated by crossing HKY894 with HKY456 and the strains HKY1369 to1372 originate from the four spores of one tetrad of the crossing of HKY734 with HKY128.

\subsection{Construction of recombinant DNA molecules}

Molecular cloning of recombinant plasmid-DNAs was performed by using the following standard molecular biological methods that were basically described previously (Sambrook et al., 1989).

\subsubsection{Polymerase chain reaction (PCR)}

To amplify specific DNA-fragments from plasmids or genomic DNA, polymerase chain reactions (PCR) with corresponding primer pairs (synthesized oligonucleotides) and thermostable DNA-polymerases were performed. For analytic PCRs, Taq-DNApolymerases ("DreamTaq" from Thermo Scientific) were used, while enzymes with proof-reading activity ("Velocity" from Bioline or "Phusion High-Fidelity" from Thermo Scientific) were preferred for usage of the PCR-product in subsequent molecular cloning reactions. The reaction conditions vary for every enzyme and were chosen as specified by the manufacturers. A standard reaction for the DreamTaq DNApolymerase is shown in Table 12. 
Table 12: Standard reaction and PCR program with DreamTaq DNA-polymerase.

The annealing temperature $(\mathrm{x})$ was chosen according to the melting temperature of the primers.

\begin{tabular}{|c|c|c|c|c|}
\hline Component & $\begin{array}{l}\text { Concentration } \\
(50 \mu \text { l reaction) }\end{array}$ & Step & Time & Temperature \\
\hline 10x DreamTaq buffer & $1 x$ & Initial denaturation & $3 \min$ & $95^{\circ} \mathrm{C}$ \\
\hline DreamTaq DNA-polymerase & $1.25 \mathrm{U}$ & Denaturation & $30 \mathrm{sec}$ & $95^{\circ} \mathrm{C}$ \\
\hline 2 mM dNTP-mix & $0.2 \mathrm{mM}$ & Annealing & $30 \mathrm{sec}$ & $x$ \\
\hline $10 \mu \mathrm{M}$ primer forward & $1 \mu \mathrm{M}$ & Extension & $1 \mathrm{~min} / \mathrm{kb}$ & $72^{\circ} \mathrm{C}$ \\
\hline $10 \mu \mathrm{M}$ primer reverse & $1 \mu \mathrm{M}$ & Final extension & $10 \mathrm{~min}$ & $72^{\circ} \mathrm{C}$ \\
\hline Template DNA & $\begin{array}{l}0.5 \mu \mathrm{l} \text { gDNA or } \\
20 \mathrm{ng} \text { plasmids }\end{array}$ & Storage & $\infty$ & $4^{\circ} \mathrm{C}$ \\
\hline
\end{tabular}

If required, PCR-products were directly purified by using the "peqGOLD Gel Extraction Kit" (Peqlab) according to the manufacturer's protocol for PCR clean-up. Otherwise, the samples were subjected to agarose gel electrophoresis.

\subsubsection{Agarose gel electrophoresis and DNA extraction}

TAE buffer ( $\mathrm{pH} 8.5): \quad 40 \mathrm{mM}$ Tris-acetate 1 mM EDTA

6x DNA loading dye: $\quad 10 \mathrm{mM}$ Tris $\mathrm{pH} 7.6$ $60 \%$ (v/v) Glycerol $60 \mathrm{mM}$ EDTA $0.03 \%(w / v)$ Bromophenol blue $0.03 \%(w / v)$ Xylene cyanol

For DNA analyses and preparation of DNA-fragments, $1 \%(w / v)$ agarose gels in 1x TAE-buffer were standardly used. To prepare the gel, $1 \mathrm{~g}$ agarose was dissolved in $100 \mathrm{ml}$ of $1 \times$ TAE buffer by heating in a microwave, cooled down and supplemented with $4 \mu \mathrm{l}$ of an ethidium bromide stock solution $(10 \mathrm{mg} / \mathrm{ml})$. Afterwards, the agarose solution was poured into a gel tray and a comb was added to form the wells for the samples. Upon polymerization, the DNA samples were mixed with 6x DNA loading dye and were loaded onto the agarose gel together with a DNA size standard (standardly $10 \mu \mathrm{l}$ of "Lambda DNA/EcoRI+HindIII Marker" from Thermo Scientific). Subsequently, gel electrophoresis was performed in $1 \mathrm{x}$ TAE-buffer at $120 \mathrm{~V}$ for 30 to 60 min and the DNA bands were examined with an UV transilluminator.

If required, specific bands were cut from the gel with a scalpel and the DNA was extracted by using the "peqGOLD Gel Extraction Kit" (Peqlab) according to the manufacturer's instruction. Finally, the DNA was eluted in $30 \mu \mathrm{l}$ of elution buffer, the concentration was measured with the NanoDrop spectrophotometer and the 
extracted DNA was stored at $-20^{\circ} \mathrm{C}$.

\subsubsection{Restriction digest and dephosphorylation of 5'DNA-ends}

Restriction digests with type II restriction endonucleases, which cleave DNA at specific palindromic recognition sites, were performed to analyze plasmids or to produce DNA-fragments with compatible ends for cloning approaches. Enzymes and their corresponding buffers and reaction conditions were used, as suggested from the manufacturers. In general for analytic reactions, $1 \mu \mathrm{g}$ of plasmid-DNA was digested in a total volume of $20 \mu \mathrm{l}$ for at least $1 \mathrm{~h}$. For preparative approaches, $5 \mu \mathrm{g}$ of plasmid-DNA was used in a reaction with a total volume of $50 \mu$ l overnight.

To avoid the re-ligation of linearized plasmid-DNAs in subsequent ligation reactions, the 5'phosphate ends from plasmid backbones were dephosphorylated. For that, $1 \mathrm{U}$ of FastAP Thermosensitive Alkaline Phosphatase (Thermo Scientific) was added directly to the reaction upon digestion of $1 \mu \mathrm{g}$ of plasmid-DNA. Upon incubation at $37^{\circ} \mathrm{C}$ for $10 \mathrm{~min}$, the enzyme was inactivated by heating at $65^{\circ} \mathrm{C}$ for $15 \mathrm{~min}$.

\subsubsection{Ligation of DNA-fragments}

To produce recombinant plasmids, the chosen DNA-fragments with compatible ends were covalently ligated by the T4 DNA ligase, which catalyzes the formation of a phosphodiester bond between the 5'-phosphate and the 3'-hydroxyl end. The reaction was performed, as suggested by the manufacturer. For one reaction $(10 \mu \mathrm{l})$, $100 \mathrm{ng}$ of plasmid backbone and twofold excess of insert were used and ligated at $16^{\circ} \mathrm{C}$ overnight.

\subsubsection{Gibson Assembly (GA) reaction}

During a Gibson Assembly reaction, multiple DNA-fragments with terminal overlapping regions can be covalently joined in an isothermal one-step reaction, which requires the following three enzymatic activities (Gibson et al., 2009). The T5 exonuclease removes nucleotides from the 5'ends of double-stranded DNAs creating single-stranded 3'overhangs. Thus, such complementary DNA sequences are able to anneal, the Phusion DNA-polymerase extends the 3'ends and the Taq DNA ligase covalently ligates the remaining gaps. Finally, recombinant plasmids are produced. The reaction was performed following the description of Gibson et al. (2009) and Gibson (2011). 
GA-master mix: $\quad 5 \%(v / v)$ PEG-800

$100 \mathrm{mM}$ Tris- $\mathrm{HCl} \mathrm{pH} 7.5$

$10 \mathrm{mM} \mathrm{MgCl} 2$

$10 \mathrm{mM}$ DTT

$200 \mu \mathrm{M}$ of each dNTP

$1 \mathrm{mM} N A D$

$0.004 \mathrm{U} / \mu \mathrm{l}$ T5 exonuclease

$0.025 \mathrm{U} / \mu \mathrm{l}$ Phusion DNA polymerase

$4 \mathrm{U} / \mu \mathrm{l}$ Taq DNA ligase

For one Gibson Assembly reaction $(20 \mu \mathrm{l}), 100 \mathrm{ng}$ of linearized plasmid backbone and twofold excess of inserts with homolog ends were diluted in $10 \mu$ of deionized, nuclease-free water and mixed with $10 \mu \mathrm{l}$ of GA-master mix. Afterwards, the sample was incubated at $50^{\circ} \mathrm{C}$ for $1 \mathrm{~h}$.

\subsubsection{Transformation of $E$. coli cells with plasmid-DNA}

The production of chemically ultra-competent E. coli cells was performed, as described by Inoue et al. (1990). For transformation with plasmids, $100 \mu$ of these competent $E$. coli cells were thawed on ice, $10 \mu$ of the ligation / Gibson assembly reaction or 100-500 ng of plasmid-DNA was added and incubated on ice for $30 \mathrm{~min}$. Then, the samples were heat-shocked at $42^{\circ} \mathrm{C}$ for $2 \mathrm{~min}$, immediately supplemented with $1 \mathrm{ml}$ of LB-medium and incubated at $37^{\circ} \mathrm{C}$ for 45 to $60 \mathrm{~min}$. This recovery phase was avoided, when plasmids carried an ampicillin resistance gene. Afterwards, the cells were pelleted by centrifugation for $5 \mathrm{~min}$ at $3500 \times \mathrm{g}$, plated onto LB-plates containing the corresponding antibiotics and incubated at $37^{\circ} \mathrm{C}$ overnight.

\subsubsection{Extraction of Plasmid-DNA from E. coli cells}

For analytic approaches, the plasmid-DNA from saturated $5 \mathrm{ml}$ bacteria cultures was extracted by using the kit "NucleoSpin Plasmid" (Macherey-Nagel), as described in the manufacturer's protocol. Finally, the DNA was eluted in $50 \mu$ of deionized, nuclease-free water.

To generate large amounts of plasmid-DNA, $100 \mathrm{ml}$ (high copy plasmids) to $200 \mathrm{ml}$ (low copy plasmids) of saturated bacteria cultures were used to isolate their plasmidDNAs with the kit "NucleoBond PC 100" (Macherey-Nagel) following the protocol of the producer. Subsequently, the DNA concentration was measured with the NanoDrop spectrophotometer and adjusted to $1 \mu \mathrm{g} / \mu \mathrm{l}$ with deionized, nuclease-free water. All plasmid-DNA was stored at $-20^{\circ} \mathrm{C}$. 


\subsubsection{Sequencing of plasmid-DNA}

All modified plasmid regions were sequenced to validate the correct DNA sequence. For that, 0.5 to $1 \mu \mathrm{g}$ of plasmid-DNA and $5 \mu \mathrm{M}$ of the sequencing primer were sent to the company LGC Genomics (Berlin/Germany).

\subsubsection{Generation of pHK1349 and pHK1380}

Plasmid pHK1349 was created by amplification of the RPL11B ORF $+900 \mathrm{bp}$ upstream of the start codon by HK1485 and HK1486 from genomic yeast DNA. The resulting PCR-product and plasmid pHK12 were digested with Sacll and Xhol. Subsequently, the plasmid backbone and the PCR-product with compatible ends were purified and ligated.

To generate plasmid pHK1380, the URA3 marker gene from pHK1323 was changed into the LEU2 gene. For that, the LEU2 gene including promoter and 3'UTR was amplified by HK1623 and HK1624 from plasmid pHK87 and in parallel, pHK1323 was digested with Pstl and Nsil. Afterwards, the purified plasmid backbone and PCRproduct with homolog ends were combined by performing a Gibson Assembly reaction.

\subsection{Molecular biological methods with yeast}

\subsubsection{Yeast cell transformation}

Lithium acetate treated yeast cells were transformed with plasmid-DNA following the description of Gietz et al. (1992) with minor modifications.

Lithium acetate/TE (pH 7.5): $\quad 100 \mathrm{mM}$ Lithium acetate $10 \mathrm{mM}$ Tris- $\mathrm{HCl}$

1 mM EDTA

PEG/Lithium acetate/TE (pH 7.5): $40 \%$ (v/v) PEG 4000

$100 \mathrm{mM}$ Lithium acetate

$10 \mathrm{mM}$ Tris- $\mathrm{HCl}$

$1 \mathrm{mM}$ EDTA

Yeast cells were grown in appropriate liquid media at $25^{\circ} \mathrm{C}$, until log-phase $\left(1-2 \times 10^{7}\right.$ cells $/ \mathrm{ml}$ ) was achieved. Then, the cells were harvested by centrifugation at $4100 \times \mathrm{g}$ for $5 \mathrm{~min}$, washed once in $1 \mathrm{ml}$ of lithium acetate/TE and finally resuspended in an appropriate volume of lithium acetate/TE to obtain a cell density of $1 \times 10^{9}$ cells $/ \mathrm{ml}$. For 
one transformation reaction, $50 \mu \mathrm{l}$ of this cell suspension $\left(5 \times 10^{7}\right.$ cells) were mixed with $1 \mu \mathrm{g}$ plasmid-DNA and $50 \mu \mathrm{g}$ Salmon sperm-carrier DNA, which was boiled for $5 \mathrm{~min}$ and cooled down on ice for $2 \mathrm{~min}$ before usage. Subsequently, $300 \mu \mathrm{l}$ of PEG/lithium acetate/TE was added to the sample, which was thoroughly mixed, incubated with agitation at $25^{\circ} \mathrm{C}$ for $30 \mathrm{~min}$ and then heat-shocked at $42^{\circ} \mathrm{C}$ for $15 \mathrm{~min}$. Afterwards, the cells were pelleted by centrifugation for $60 \mathrm{sec}$ at $21100 \times \mathrm{g}$, the supernatant was removed and the cells were resuspended in $100 \mu \mathrm{l}$ of sterile water to plate them onto a selective agar plate. Finally, the plates were incubated at $25^{\circ} \mathrm{C}$ for two to three days and subsequently, growing single colonies were streaked onto new selective agar plates.

\subsubsection{Extraction of chromosomal DNA from yeast cells}

Chromosomal / genomic DNA (gDNA) from yeast cells was extracted, as described previously (Rose et al., 1990).

Detergent lysis buffer (pH 8.0): $2 \%(\mathrm{v} / \mathrm{v})$ Triton X-100

$1 \%(w / v)$ SDS

$100 \mathrm{mM} \mathrm{NaCl}$

$10 \mathrm{mM}$ Tris- $\mathrm{HCl}$

1 mM EDTA

TE-buffer (pH 8.0): $\quad 10 \mathrm{mM}$ Tris-HCl

$1 \mathrm{mM}$ EDTA

Yeast cells were grown in $10 \mathrm{ml}$ liquid cultures to saturation and collected by centrifugation at $4100 \times \mathrm{g}$ for $5 \mathrm{~min}$. Upon washing once with water, the pellet was resuspended in $100 \mu \mathrm{l}$ of $\mathrm{H}_{2} \mathrm{O}$ and $200 \mu \mathrm{l}$ of detergent lysis buffer, $200 \mu \mathrm{l}$ of phenol/chloroform/isoamyl alcohol (25:24:1) (P/C/I) and $300 \mu \mathrm{l}$ of glass beads were added. The cells were disrupted by using twice the FastPrep-24 machine at $5 \mathrm{~m} / \mathrm{s}$ for $20 \mathrm{sec}$. Subsequently, $200 \mu \mathrm{l}$ of TE-buffer was added and the phases were separated by centrifugation for $5 \mathrm{~min}$ at $21100 \times \mathrm{g}$ and room temperature (RT). Then, the upper phase was transferred into a fresh tube and the same amount of $\mathrm{P} / \mathrm{C} / \mathrm{l}$ was added, vigorously mixed and centrifuged again for $5 \mathrm{~min}$ at $21100 \mathrm{xg}$ and RT. This procedure was repeated, until the interphase remained clear. Finally, the chromosomal DNA from the upper phase was precipitate by the addition of $6 \mu \mathrm{l}$ of $7.5 \mathrm{M}$ ammonium acetate and $1 \mathrm{ml}$ of $100 \%$ ethanol and centrifugation for $10 \mathrm{~min}$ at $21100 \times \mathrm{g}$ and $4^{\circ} \mathrm{C}$. Afterwards, the precipitate DNA was washed once with $70 \%$ 
ethanol, air dried and resuspended in 50-100 $\mu$ l of deionized water. Storage was performed at $-20^{\circ} \mathrm{C}$.

\subsubsection{Extraction of total RNA from yeast cells}

Yeast cells were grown in $50 \mathrm{ml}$ of YPD medium to log-phase ( $2 \times 10^{7}$ cells $\left./ \mathrm{ml}\right)$, shifted for $1 \mathrm{~h}$ to their restrictive temperatures and harvested by centrifugation for $5 \mathrm{~min}$ at $2050 \times \mathrm{g}$ and $4^{\circ} \mathrm{C}$. The total RNA of these pellets was extracted by using the kit "NucleoSpin RNA" (Macherey-Nagel) following the manufacturer's protocol that includes the DNase I treatment. Finally, the RNA was eluted in $60 \mu$ of nucleasefree water, the concentration was measured with the NanoDrop spectrophotometer and adjusted to $1 \mu \mathrm{g} / \mu \mathrm{l}$ (if possible). RNA samples were stored at $-80^{\circ} \mathrm{C}$.

\subsubsection{Synthesis of digoxigenin (DIG)-labeled RNA-probes}

Digoxigenin (DIG)-labeled RNA-probes were needed for the detection of specific RNAs in fluorescence in situ hybridization experiments (see section 5.11.3) and in non-radioactive Northern blot analyses (see section 5.10.5). The synthesis of these probes was performed, as suggested by the manufacturer of the DIG-labeling mix (Roche Diagnostics, DIG Application Manual for Filter Hybridization, 2008).

The DIG-labeled RNA-probes that were designed in this study for the recognition of ribosomal RNAs detect always their precursor molecules as well, as these molecules arise in a series of cleavage events from the common 35S precursor (see Figure 1B). Thus, the 20 s pre-rRNA probe is complementary to the 5'ITS1 and the 27S pre-rRNA probe to the ITS2, but both recognize also 35S, 33S and 32S pre-rRNAs. The mature rRNA probes detect all precursor molecules.

To produce templates for the synthesis of specific RNA-probes, PCR-products with a T7-RNA-polymerase promoter on the antisense strand were generated by using the primer pairs HK1138+1139 (25S rRNA probe), HK1140+1141 (18S rRNA probe), HK1893+1894 (20S pre-rRNA probe), HK1895+974 (27S pre-rRNA probe) and HK1723+1724 (U2 snRNA probe) in a PCR reaction with gDNA and were purified from agarose gels with the "peqGOLD Gel Extraction Kit" (Peqlab). These purified PCR-products were used as templates in an in vitro transcription assay with the T7RNA-polymerase and the "DIG RNA labeling mix" (Roche) to generate antisense RNA probes. In such reaction, DIG-11-UTP will be incorporated in average every $20^{\text {th }}$ to $25^{\text {th }}$ nucleotide in the newly synthesized RNA. The following reaction mix $(20 \mu \mathrm{l})$ was incubated for $2 \mathrm{~h}$ at $37^{\circ} \mathrm{C}$ : 
250 ng template (purified PCR product)

1x DIG RNA labeling mix (Roche)

20 U RiboLock RNase Inhibitor (Thermo Scientific)

1x Transcription buffer (Thermo Scientific)

40 U T7-RNA-polymerase (Thermo Scientific)

Afterwards, the produced RNA was precipitated by LiCl-precipitation (for $>100 \mathrm{nt}$ ).

Hyb-Mix: $\quad 50 \%(\mathrm{v} / \mathrm{v})$ deionized formamide

$5 \times$ SSC

1x Denhardt's

$0.1 \mathrm{mg} / \mathrm{ml}$ Heparin

Filled up with DEPC-treated water

50x Denhardt's: $\quad 1 \%(w / v)$ Ficoll

$1 \%(w / v)$ Polyvinylpyrrolidone

$1 \%(w / v)$ BSA

For precipitation, the reaction mix was filled up to $50 \mu$ with RNase-free water and $1 / 4$ volume of $4 \mathrm{M} \mathrm{LiCl}, 100 \mu \mathrm{g}$ of tRNA and 3 volumes of $100 \%$ ethanol was added and incubated overnight at $-20^{\circ} \mathrm{C}$. Then, the RNA was pelleted by centrifugation for $60 \mathrm{~min}$ at $21100 \times \mathrm{g}$ and $4^{\circ} \mathrm{C}$, washed with $70 \%$ ethanol, dried on ice and resuspended in $25 \mu \mathrm{l}$ of $1 \mathrm{M} \mathrm{TE} \mathrm{pH} 7.5+1 \mathrm{mg} / \mathrm{ml}$ heparin. Afterwards, $25 \mu \mathrm{l}$ of deionized formamide and $50 \mu \mathrm{l}$ of Hyb-Mix were added and the RNA-probes were stored at $-20^{\circ} \mathrm{C}$.

\subsubsection{RNA-formaldehyde gels and Northern blotting}

To analyze the steady state level of ribosomal RNAs in different yeast strains, their total RNA was extracted (see section 5.10.3) separated on denaturing RNAformaldehyde agarose gels and analyzed by Northern blotting, as basically described previously (Sambrook et al., 1989; Wu et al., 2014). Non-radioactive Northern blotting with DIG-labeled RNA probes was performed according to the suggestions of the manufacturer (Roche Diagnostics, DIG Application Manual for Filter Hybridization, 2008).

Before starting the experiment, all solid materials were incubated with $0.1 \mathrm{M} \mathrm{NaOH}$ for $30 \mathrm{~min}$ and all solutions were treated with diethyl dicarbonate (DEPC) to inactivate RNases and prevent RNA degradation. For that, $1 \mathrm{ml}$ of DEPC was added to 1 I deionized water, stirred overnight and subsequently autoclaved. 
10x MOPS (pH 7.0): $\quad 200 \mathrm{mM}$ MOPS

$50 \mathrm{mM}$ Sodium acetate

10 mM EDTA

20x SSC (pH 7.0): $\quad 3 \mathrm{M} \mathrm{NaCl}$

$300 \mathrm{mM}$ Sodium citrate

Hybridization buffer (pH 7.2): $\quad 0.5 \mathrm{M} \mathrm{Na-phosphate} \mathrm{pH} 7.2$

$7 \%(w / v)$ SDS

$1 \mathrm{mM}$ EDTA

1M Na-Phosphate buffer (pH 7.2): $68.4 \mathrm{ml} 1 \mathrm{M} \mathrm{Na}_{2} \mathrm{HPO}_{4}$

$31.6 \mathrm{ml} 1 \mathrm{M} \mathrm{NaH}_{2} \mathrm{PO}_{4}$

5x Maleic acid buffer ( $\mathrm{pH} 7.5): \quad 0.5 \mathrm{M}$ Maleic acid

$0.75 \mathrm{M} \mathrm{NaCl}$

10x Blocking stock solution: $\quad 10 \%(w / v)$ Blocking reagent (Roche)

$1 \times$ Maleic acid buffer

1x Blocking buffer: $10 x$ Blocking stock solution diluted in 1x Maleic acid buffer (freshly prepared before use)

Washing buffer: $\quad 1 x$ Maleic acid buffer

$0.3 \%(v / v)$ Tween 20

Detection buffer: $\quad 0.1 \mathrm{M}$ Tris $\mathrm{pH} 9.5$

$0.1 \mathrm{M} \mathrm{NaCl}$

RNA Loading Dye: $50 \%(\mathrm{v} / \mathrm{v})$ deionized formamide

$6 \%(\mathrm{v} / \mathrm{v})$ Formaldehyde

1x MOPS

$25 \mathrm{ng} / \mathrm{ml}$ Ethidium bromide

10\% Glycerol (RNase-free)

Bromophenol blue and Xylene cyanol

At the beginning, a $1 \%$ agarose gel containing $2 \%$ formaldehyde was poured under the hood. For that, $1.5 \mathrm{~g}$ agarose was dissolved in $127 \mathrm{ml}$ of nuclease-free water, cooled down to $55^{\circ} \mathrm{C}$ and mixed with pre-warmed $15 \mathrm{ml}$ of $10 x$ MOPS and $8.1 \mathrm{ml}$ of formaldehyde (37\% from Applichem). During polymerization, the RNA samples were prepared. For each strain $1 \mu \mathrm{g}$ of total RNA was diluted in $5 \mu \mathrm{l}$ of DEPC-water, mixed with $10 \mu \mathrm{l}$ of freshly prepared RNA-Loading Dye, denatured for $10 \mathrm{~min}$ at $65^{\circ} \mathrm{C}$ and chilled on ice. The complete samples were loaded into the wells of the formaldehydeagarose gel, which was subsequently run in 1 x MOPS at $80 \mathrm{~V}$ for $4-5 \mathrm{~h}$. Afterwards, the quality of the RNA was examined with an UV transilluminator. To remove the formaldehyde before Northern blotting, the gel was washed once in DEPC-water and twice in 20x SSC for 15 min. A dry Northern blot was performed overnight and the following sandwich was set up: 
(1) the gel facing down on a glass plate and wrapped with parafilm

(2) a dry, positively charged nylon membrane (Amersham Hybond- $\mathrm{N}^{+}$) with the size of the gel

(3) three dry Whatman papers $(0.8 \mathrm{~mm})$ in the same size

(4) a stack of paper towels and a 200-500 g weight

The next day, the RNA was cross-linked by placing the membrane on a Whatman paper pre-soaked with 2x SSC and exposing it to UV light for $7 \mathrm{~min}$ at $5000 \mathrm{~J} / \mathrm{cm}^{2}$ and afterwards, by baking the membrane for $2 \mathrm{~h}$ at $80^{\circ} \mathrm{C}$. Then, the membrane was stored dry or was pre-hybridized with hybridization buffer in a closed glass tube for $1 \mathrm{~h}$ at $68^{\circ} \mathrm{C}$. For one detection, $1 \mu \mathrm{l}$ of each DIG-labeled RNA probe (for preparation see section 5.10.4) was pipetted in $5 \mu$ l of hybridization buffer, denatured for 5 min at $55^{\circ} \mathrm{C}$ and added to the hybridization solution with the membrane. Hybridization was done at $68^{\circ} \mathrm{C}$ overnight. Afterwards, the following washing steps were performed for 15 min each: in $2 \times$ SSC/ $0.1 \%$ SDS at RT, in $1 \times$ SSC/ $0.1 \%$ SDS at RT and twice in $0.5 \mathrm{xSC} / 0.1 \% \mathrm{SDS}$ at $68^{\circ} \mathrm{C}$. For detection, the membrane was washed for $5 \mathrm{~min}$ in washing buffer, blocked for $30 \mathrm{~min}$ in $1 x$ blocking buffer and incubated for 30-60 min with anti-Digoxigenin-alkaline phosphatase, Fab fragments (diluted 1:10 000 in blocking buffer, from Roche). After washing twice for 15 min in washing buffer, the membrane was equilibrated for 2-5 min in detection buffer and incubated with the chemiluminescent substrate CSPD (diluted 1:100 in detection buffer, from Roche) for $5 \mathrm{~min}$. The membrane was enclosed in an autoclave bag and incubated for $10 \mathrm{~min}$ at $37^{\circ} \mathrm{C}$ to reduce the background. To detect the emitted light, the membrane was exposed to X-ray films (Fuji), which were subsequently developed with an X-ray film processor. For hybridization with an additional DIG-labeled RNA-probe, the procedure was repeated starting at the pre-hybridization step.

\subsection{Cell biological methods with yeast}

\subsubsection{Fluorescence microscopy}

In general, formaldehyde-fixed yeast cells with fluorescent signals were examined with a Leica DMI6000B fluorescence microscope and pictures were obtained by using the Leica DFC360 FX camera and the LAS AF 1.6.2 software. To avoid movement of the cells, they were transferred onto microscope slides that were previously coated with $0.3 \%(\mathrm{w} / \mathrm{v})$ poly-L-lysine hydrobromide. For preparation of the 
slides, the polylysine solution was dropped onto each well of the microscope slide, dried on $\mathrm{a} 65^{\circ} \mathrm{C}$ heating block and subsequently washed with water.

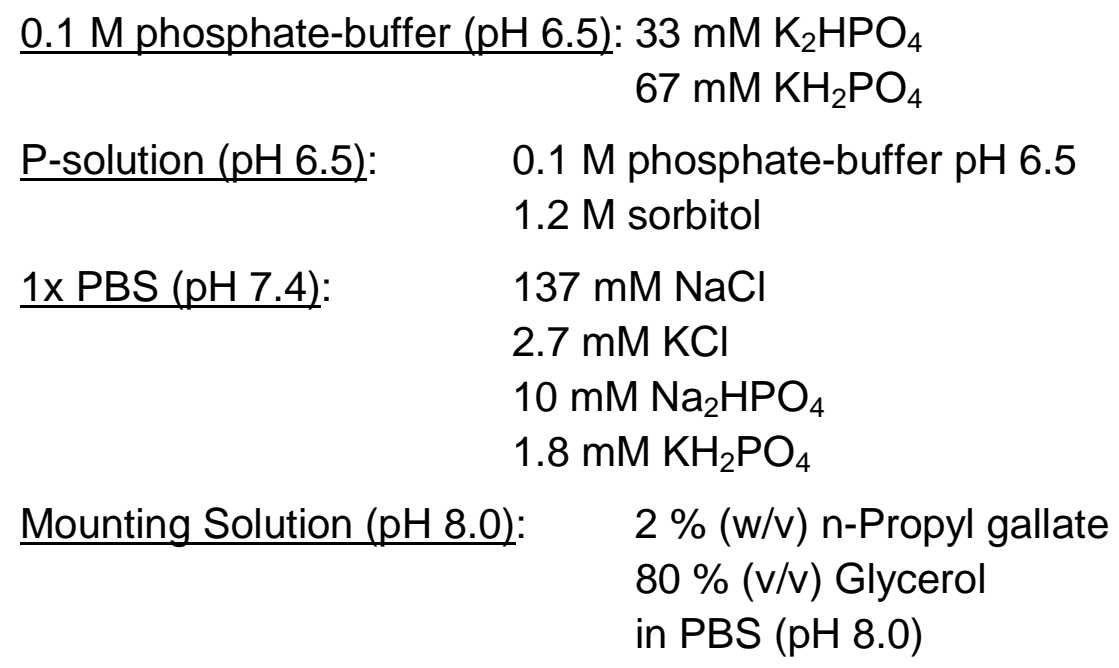

\subsubsection{GFP-microscopy}

The cellular localization of GFP-tagged proteins was examined in formaldehyde-fixed cells, as described previously (Windgassen and Krebber, 2003). For that, GFPfusion proteins expressing yeast cells were grown in selective media to log-phase, shifted for $1 \mathrm{~h}$ to their non-permissive temperatures and shortly fixed with $2.6 \%(\mathrm{v} / \mathrm{v})$ formaldehyde. To prevent destruction of the fluorescent GFP, the cells were immediately centrifuged for $5 \mathrm{~min}$ at $2050 \times \mathrm{g}$ and $4^{\circ} \mathrm{C}$ and washed once with $0.1 \mathrm{M}$ phosphate-buffer and once with P-solution. Depending on the size of the pellet, the cells were resuspended in $50-150 \mu \mathrm{l}$ of P-solution and $20 \mu \mathrm{l}$ of this suspension was transferred onto one well of the polylysine-coated microscope slide. After $20 \mathrm{~min}$ incubation, the cells were permeabilized by treatment with $0.5 \%(\mathrm{v} / \mathrm{v})$ TritonX-100 in P-solution for $5 \mathrm{~min}$. Upon washing with 1xPBS, the DNA was stained with Hoechst 33342 (diluted 1:10 000 in PBS) for 5 min followed by two washing steps with 1x PBS. Finally, the slides were dried and sealed with mounting solution and a cover slip.

\subsubsection{Fluorescence in situ hybridization (FISH)}

Fluorescence in situ hybridization (FISH) is a method to localize specific nucleic acids such as DNAs and RNAs in tissues or cells by using fluorescent labeled probes that specifically hybridize with the nucleic acids of interest. To localize the different ribosomal RNAs in yeast cells, FISH experiments with specific DIG-labeled RNA- 
probes (generation see section 5.10.4) were performed according to Amberg et al. (1992) with some modifications. Furthermore, a Cy3-end labeled oligo(dT) 50 probe was used for detection of poly $(A)^{+}$RNAs.

All buffers and solutions were prepared with DEPC-treated water.

Hyb-Mix: $\quad$ see section 5.10.4

20x SSC: $\quad$ see section 5.10 .5

Zymolyase-Solution: $\quad 10 \mathrm{mg} / \mathrm{ml}$ Zymolyase

$2 \mathrm{mM}$ Vanadyl ribonucleoside complexes

$1 \mathrm{mg} / \mathrm{ml}$ Heparin

Antibody blocking buffer (ABB): 1x PBS

$5 \%(w / v)$ heat-inactivated FBS

$0.3 \%(v / v)$ Triton-X100

Liquid cultures with log-phase yeast cells were shifted to their restrictive temperatures or induced by galactose addition as indicated. Subsequently, the cells were fixed by addition of $4 \%(v / v)$ formaldehyde and incubation with agitation at RT for $1 \mathrm{~h}$ and then pelleted by centrifugation for $5 \mathrm{~min}$ at $2050 \mathrm{xg}$ and $4^{\circ} \mathrm{C}$. Upon washing three times with $1 \mathrm{ml} \mathrm{P}$-solution, the cells were resuspended in $100 \mu \mathrm{l}$ of P-solution and treated with zymolyase to digest the cell wall and produce spheroplasts. For that, the cell suspension was incubated with $10 \mathrm{mM}$ DTT for $10 \mathrm{~min}$ at RT and subsequently $50 \mu \mathrm{g}$ zymolyase was added. The enzymatic reaction was performed at RT and its progress was followed by examination with a light microscope. When half of the cells appeared dark indicating a successful cell wall digestion, $1 \mathrm{ml}$ of P-solution was added to stop the reaction. The spheroplasts were carefully pelleted by centrifugation for $3 \mathrm{~min}$ at $400 \times \mathrm{g}$ and $4^{\circ} \mathrm{C}$ and washed two times in P-solution. Afterwards, the spheroplasts were resuspended in an appropriate volume of Psolution $(-100 \mu \mathrm{l})$ and $25 \mu \mathrm{l}$ of the suspension were transferred onto one well of the polylysine-coated microscope slides. Upon 15 min incubation, the adhered spheroplasts were permeablized with $0.5 \%(\mathrm{v} / \mathrm{v})$ triton X-100 in P-solution for $10 \mathrm{~min}$ at RT and rinsed with P-solution. Subsequently, equilibration in $0.1 \mathrm{M}$ triethanolamine $\mathrm{pH} 8.0$ was performed for $2 \mathrm{~min}$ and the polar groups of proteins were blocked with $0.25 \%(\mathrm{v} / \mathrm{v})$ acetic anhydride in $0.1 \mathrm{M}$ triethanolamine $\mathrm{pH} 8.0$ for $10 \mathrm{~min}$ at RT. Upon rinsing with P-solution, the cells were pre-hybridized with Hyb-mix supplemented with $500 \mu \mathrm{g} / \mathrm{ml}$ of tRNAs and $500 \mu \mathrm{g} / \mathrm{ml}$ of denatured Salmon sperm carrier-DNAs for $1 \mathrm{~h}$ at $37^{\circ} \mathrm{C}$. Afterwards, hybridization with specific probes was performed overnight at 
$37^{\circ} \mathrm{C}$. For that, DIG-labeled RNA probes $(0.1 \mu \mathrm{l} \text { per well) or Cy3-labeled oligo(dT) })_{50}$ probes $(0.5 \mu \mathrm{l}$ per well) in tRNA and SsDNA supplemented Hyb-mix were added to each well. The next day, the following washing steps were performed for 30-60 min each: $2 \times \mathrm{SSC}$ at RT, $1 \times \mathrm{SSC}$ at RT, $0.5 \times \mathrm{SSC}$ at $37^{\circ} \mathrm{C}$ and $0.5 \times \mathrm{SSC}$ at RT. Upon blocking with ABB for $1 \mathrm{~h}$ at RT, a sheep anti-digoxigenin Fab-FITC antibody (Roche) was diluted 1:200 in $\mathrm{ABB}$ and added overnight at $4^{\circ} \mathrm{C}$ for DIG detection. Then, the following washing steps were performed at RT: twice with ABB for 15 min, once with ABB for 30 min and twice with $0.1 \%$ (v/v) Tween-20 in PBS for 30 min. Afterwards, the DNA was stained with Hoechst 33342 (diluted 1:10 000 in PBS) for 5 min and three times washed with PBS. Finally, the slides were air dried and sealed with mounting-solution and cover slips.

\subsubsection{Statistical analyses}

The ratio of cells that showed a nuclear accumulation of the fluorescent signal was determined from several microscopy pictures and subsequently, the average was calculated for every strain.

To determine the average enrichment of the nuclear signal, the fluorescent signals of the whole cell and of its nucleus of at least 10 cells with phenotype per strain were quantified by using the Fiji software. Subsequently, the intensity of the nuclear signal was related to the complete cell and to the ratio of the wild type. The significance of the enrichment was calculated by performing an unpaired Student's t-test (type 2) with the Microsoft Excel software.

\subsection{Protein biochemical methods}

\subsubsection{Preparation of yeast whole cell lysates}

In general, pellets of log-phase yeast cells (see section 5.7.2) were used to generate cell lysates. All steps were performed on ice to avoid degradation. According to the needed amount and the pellet size, the cells were transferred into $2 \mathrm{ml}$ screw-cap eppi tubes or $15 \mathrm{ml}$ falcon tubes. For cell lysis, the same amounts of glass beads and of the assay-specific lysis buffer supplemented with Complete, EDTA-free protease inhibitor cocktail (Roche) were added to the cell pellets, which were homogenized at least two times in a FastPrep-24 machine at $5 \mathrm{~m} / \mathrm{s}$ for $20 \mathrm{sec}$. Afterwards, the lysates were centrifuged twice for $10 \mathrm{~min}$ at $21100 \times \mathrm{g}$ and $4^{\circ} \mathrm{C}$ and the cleared lysates were immediately used for following applications. 


\subsubsection{Co-immunoprecipitation studies for analyses of protein-protein}

\section{interactions in vivo}

To analyze protein-protein interactions in vivo, co-immunoprecipitation studies with GFP- or TAP-tagged proteins expressing yeast cells were performed, as basically described previously (Gross et al., 2007). The tandem affinity purification (TAP)-tag consists of the calmodulin-binding peptide (CBP), a TEV cleavage site and two IgGbinding units of protein A of Staphylococcus aureus (ProtA) (Rigaut et al., 1999). Thus, fusion proteins can be immunoprecipitated with IgG-Sepharose beads, which bind with high affinity ProtA. For the immunoprecipitation of GFP-tagged proteins, GFP-Trap_A beads (Chromotek) containing covalently bound GFP-binding proteins were used.

\section{PBSKMT-buffer: $\quad$ 1xPBS \\ $3 \mathrm{mM} \mathrm{KCl}$ \\ $2.5 \mathrm{mM} \mathrm{MgCl}_{2}$ \\ 0.5\% (v/v) Triton-X 100}

Yeast cell pellets from $300-500 \mathrm{ml}$ cultures were lyzed with PBSKMT-buffer supplemented with protease inhibitor cocktail for yeast (Sigma-Aldrich) and Complete, EDTA-free protease inhibitor cocktail (Roche), as described in section 5.12.1. As input control, at least $25 \mu \mathrm{l}$ of the lysates were mixed with 2x SDS-sample buffer and stored at $-20^{\circ} \mathrm{C}$ for subsequent SDS-PAGE. For immunoprecipitation of GFP-tagged proteins, $10 \mu$ slurry of GFP-Trap_A beads (Chromotek) and for TAPtagged proteins, $20 \mu \mathrm{l}$ slurry of IgG-Sepharose beads (GE Healthcare) were used per reaction and washed at least four times with $1 \mathrm{ml}$ of PBSKMT-buffer. Then, 500$1000 \mu \mathrm{l}$ of the cleared lysates were added and incubated for $3-4 \mathrm{~h}$ at $4^{\circ} \mathrm{C}$ on an overhead rotator. If indicated, the samples were treated with $0.2 \mathrm{mg} / \mathrm{ml}$ RNase A (AppliChem) for $30 \mathrm{~min}$ at $4^{\circ} \mathrm{C}$ to degrade single-stranded RNAs. Afterwards, the beads were washed six times with $1 \mathrm{ml}$ of PBSKMT-buffer, whereby the centrifugation in between was performed at low speed ( 2 min at $400 \times \mathrm{g}$ and $4^{\circ} \mathrm{C}$ ). For elution, the beads were mixed with $25 \mu$ of $2 x$ SDS-sample buffer and boiled for $5 \mathrm{~min}$ at $95^{\circ} \mathrm{C}$. Subsequently, the samples were stored at $-20^{\circ} \mathrm{C}$ or immediately loaded along with $15 \mu \mathrm{l}$ of the input samples onto $10 \%$ SDS-polyacrylamide gels to separate the eluted proteins and to perform Western blot analyses (see section 5.12.6 and 5.12.8). 


\subsubsection{Expression of recombinant proteins and affinity purification for analyses of protein-protein interactions in vitro}

To analyze the direct binding of yeast proteins, in vitro binding studies were performed with recombinant proteins that were expressed in E. coli Rosetta 2 (DE3) cells (Novagen). These cells provide the T7 expression system and contain a plasmid that carries a chloramphenicol-resistance gene and expresses tRNAs for rarely used codons in E. coli. Thus, expression of eukaryotic proteins needing a different codon usage can be improved. The expression of Glutathione S-transferase (GST)- and $\mathrm{His}_{6}$-tagged recombinant yeast proteins and the subsequent affinity purification in batch mode was performed according to standard protocols (Block et al., 2009; Harper and Speicher, 2011).

Lysis buffer: $20 \mathrm{mM}$ HEPES pH 7.5

$100 \mathrm{mM} \mathrm{NaCl}$

$4 \mathrm{mM} \mathrm{MgCl}_{2}$

$20 \%(\mathrm{v} / \mathrm{v})$ Glycerol

$0.5 \%(\mathrm{v} / \mathrm{v}) \mathrm{NP}-40$

$1 \mathrm{mM}$ DTT

Complete, EDTA-free protease inhibitor cocktail (Roche)

His-Mtr2 and Mex67 were co-expressed and purified together by affinity chromatography with Protino Ni-IDA Resin (Macherey-Nagel) that was performed by Lysann Henker and Michael Mitter (laboratory of Prof. Heike Krebber) according to Yao et al. (2007). The proteins were subsequently stored at $-80^{\circ} \mathrm{C}$ in elution buffer (20 mM HEPES pH 7.4, 100 mM KCl, 10 mM NaCl, 4 mM MgCl $2,20 \%$ (v/v) Glycerol, $0.5 \%$ (v/v) NP-40, Complete, EDTA-free protease inhibitor cocktail).

To induce expression of GST-tagged proteins, $50 \mathrm{ml}$ of LB-medium supplemented with $100 \mu \mathrm{g} / \mathrm{ml}$ of ampicillin and $34 \mu \mathrm{g} / \mathrm{ml}$ of chloramphenicol were inoculated with single colonies of E. coli Rosetta 2 cells containing GST or GST-DBP5 expressing plasmids and incubated at $37^{\circ} \mathrm{C}$ overnight. The next day, $100 \mathrm{ml}$ of $2 x Y T$ medium supplemented with the same antibiotics were inoculated with $2 \mathrm{ml}$ of the pre-cultures and incubated at $37^{\circ} \mathrm{C}$, until an $\mathrm{OD}_{600}$ of 1.2 was achieved. Then, $0.5 \mathrm{mM}$ IPTG was added to induce the expression from the tac-promoter. In parallel, $100 \mathrm{ml}$ of precooled medium $\left(4^{\circ} \mathrm{C}\right)$ were added to enhance the expression of chaperons, which support the correct folding of the induced proteins. Induction was performed overnight at $16^{\circ} \mathrm{C}$. The next day, the cells were harvested à $50 \mathrm{ml}$ fractions by centrifugation for $10 \mathrm{~min}$ at $4200 \times \mathrm{g}$ and $4^{\circ} \mathrm{C}$ and the pellets were stored at $-20^{\circ} \mathrm{C}$. 
Before and after induction a sample of $1 \mathrm{OD}_{600}$ was taken, centrifuged for $1 \mathrm{~min}$ at $21100 \times \mathrm{g}$ and the cell pellet was resuspended in $25 \mu \mathrm{l}$ of $2 \times$ SDS-sample buffer. To proof successful expression, $10 \mu$ of the samples were loaded onto a $10 \%$ SDSpolyacrylamide gel, which was stained with Coomassie upon SDS-PAGE (see section 5.12.6 and 5.12.7).

For in vitro binding studies, the bacteria pellets (each from $50 \mathrm{ml}$ media) were resuspended in $4 \mathrm{ml}$ of pre-cooled lysis buffer and sonified twice for $5 \mathrm{~min}$ to break the cells. Afterwards, the crude extracts were centrifuged for $20 \mathrm{~min}$ at $21100 \mathrm{xg}$ and $4^{\circ} \mathrm{C}$ to clear the lysates. Then, $100 \mu$ l of GST or GST-Dbp5 containing lysates were incubated with $15 \mu$ l slurry of Glutathione Sepharose 4B beads (GE Healthcare), which had been washed before three times with lysis buffer. After rotation on an over-head rotator for $1 \mathrm{hr}$ at $4^{\circ} \mathrm{C}, 30 \mu \mathrm{g}$ of the purified heterodimer HisMtr2-Mex67 was added and incubated for $1 \mathrm{~h}$ at $4^{\circ} \mathrm{C}$. Then, the beads were extensively washed seven times with $1 \mathrm{ml}$ of lysis buffer and the bound proteins were eluted with $50 \mu \mathrm{l}$ of $2 \times \mathrm{SDS}$-sample buffer and boiled for $5 \mathrm{~min}$ at $95^{\circ} \mathrm{C}$. Half of the eluates were subjected to SDS-PAGE and Western blotting or Coomassie staining, respectively. In addition, $3.5 \mu \mathrm{l}$ of the GST and GST-Dbp5 containing lysates as well as $6 \mu \mathrm{g}$ of the purified heterodimer His-Mtr2-Mex67 were mixed with the same amount of 2x SDS-sample buffer and also loaded onto the SDS-polyacrylamide gel as input control.

\subsubsection{Sucrose-density gradient fractionation}

To separate different ribosomal complexes according to their size, sucrose-density gradient fractionation experiments were performed, as described previously (Frey et al., 2001; Masek et al., 2011) with some modifications.

Lysis buffer: $\quad 20 \mathrm{mM}$ HEPES-KOH pH 7.5

$10 \mathrm{mM} \mathrm{KCl}$ (or alternatively $100 \mathrm{mM}$ )

$2.5 \mathrm{mM} \mathrm{MgCl}_{2}$

$1 \mathrm{mM}$ EGTA

$1 \mathrm{mM}$ DTT

$100 \mu \mathrm{g} / \mathrm{ml}$ Cycloheximide

Sucrose Solutions: 7 or $47 \%(\mathrm{w} / \mathrm{v})$ sucrose

20 mM HEPES-KOH pH 7.5

$10 \mathrm{mM} \mathrm{KCl}$ (or alternatively $100 \mathrm{mM}$ )

$2.5 \mathrm{mM} \mathrm{MgCl}_{2}$ 
Yeast cells were grown in $200 \mathrm{ml}$ of appropriate medium to log-phase overnight and were subsequently shifted to their restrictive temperatures for the indicated time periods. To retain the polysomes, cycloheximide (Carl Roth) was added to a final concentration of $100 \mu \mathrm{g} / \mathrm{ml}$ and incubated for $15 \mathrm{~min}$ on ice. Afterwards, the cells were harvested and lyzed in pre-cooled lysis buffer supplemented with Complete, EDTA-free protease inhibitor cocktail (Roche), as described in section 5.12.1. If indicated, the pre-cleared lysates were treated with $0.25 \mathrm{mg} / \mathrm{ml}$ RNase A (Applichem) or with $100 \mathrm{mM}$ EDTA pH 8.0 for 20-30 min on ice.

Linear sucrose-gradients with a volume of $12 \mathrm{ml}$ were poured with the gradient master and cooled on ice until usage. Standardly, $7 \%(\mathrm{w} / \mathrm{v})$ sucrose was used for the low concentrated and $47 \%(\mathrm{w} / \mathrm{v})$ sucrose for the high concentrated sugar solution, so that linear 7-47 \% sucrose-gradients were generated upon mixture. To load the same amount of yeast lysate from each strain, the absorption at $260 \mathrm{~nm}$ was measured with the NanoDrop spectrophotometer. For protein analyses, $10-30 \mathrm{OD}_{260}$ units and for ribosomal profile analyses $5-7 \mathrm{OD}_{260}$ units of the lysates were loaded on top of the linear sucrose-gradients and separated by centrifugation for $3 \mathrm{~h}$ at $40000 \mathrm{rpm}$ (average $201149 \times \mathrm{g}$ ) and $4^{\circ} \mathrm{C}$ in a TH-641 rotor and a Sorvall WX80 ultracentrifuge (Thermo Scientific). Afterwards, the gradients were fractionated in $600 \mu \mathrm{l}$ fractions with a fraction collector (Teledyne Isco) by pumping $60 \%$ (w/v) sucrose dissolved in water into the system. During fractionation, the absorbance at $254 \mathrm{~nm}$ was documented to follow the distribution of the ribosomal RNAs and to obtain polysomal profiles. Afterwards, the fractions were stored at $-20^{\circ} \mathrm{C}$ until usage.

To analyze the associated proteins of each fraction, all proteins were precipitated with trichloroacetic acid, as described in sections 5.12.5. Subsequently, the samples were subjected to SDS-PAGE and Western blotting. Some fractions were pooled as indicated to be able to load the complete gradient onto one SDS-polyacrylamide gel. From the ribosomal profiles of EDTA treated cells, the 60S/40S ratios were determined from at least three independent experiments and the average value was calculated for each strain. For that, the Fiji software was used to calculate the area underneath the $40 \mathrm{~S}$ and $60 \mathrm{~S}$ peaks and both values were set into relation.

\subsubsection{Protein precipitation}

To precipitate proteins from aqueous solutions, the same volume of $20 \%(\mathrm{w} / \mathrm{v})$ trichloroacetic acid (TCA) was added, vigorously mixed and shortly incubated on ice. Subsequently, the precipitated proteins were pelleted by centrifugation for $15 \mathrm{~min}$ at 
$21100 \times \mathrm{g}$ and $4^{\circ} \mathrm{C}$, the supernatants were removed and the pellets were washed twice with $80 \%(\mathrm{v} / \mathrm{v})$ acetone to remove the acid. Afterwards, the pellets were dried at RT and extensively resuspended in $30 \mu \mathrm{l}$ of $2 x$ SDS-sample buffer.

\subsubsection{SDS-polyacrylamide gel-electrophoresis (SDS-PAGE)}

For protein separation, denaturing SDS-polyacrylamide gel-electrophoresis (SDSPAGE) was performed, as basically described by Garfin (2009). A discontinuous Trisglycine-buffer based and denaturing system according to Laemmli (1970) was used with SDS-polyacrylamide gels that standardly consist of a $5 \%$ stacking gel and of a $10 \%$ resolving gel.

Stacking gel (5\%): $\quad 16.7 \%(v / v)$ Rotiphorese Gel 30

$125 \mathrm{mM}$ Tris- $\mathrm{HCl} \mathrm{pH} 6.8$

$0.1 \%(w / v)$ SDS

$0.1 \%(\mathrm{w} / \mathrm{v})$ Ammonium persulfate (APS)

$0.1 \%(\mathrm{v} / \mathrm{v}) \mathrm{N}, \mathrm{N}, \mathrm{N}$ ',N'-Tetramethylenediamine (TEMED)

Resolving gel (10\%): $\quad 33.3 \%$ (v/v) Rotiphorese Gel 30

$375 \mathrm{mM}$ Tris- $\mathrm{HCl}$ pH 8.8
$0.1 \%(w / v)$ SDS
$0.1 \%(w / v)$ APS
$0.04 \%(\mathrm{v} / \mathrm{v})$ TEMED

Running Buffer: $\quad 25 \mathrm{mM}$ Tris

$192 \mathrm{mM}$ Glycine

$0.1 \%(w / v)$ SDS

3x SDS-sample buffer: $\quad 300 \mathrm{mM}$ Tris- $\mathrm{HCl}$ pH 6.8

$30 \%$ (v/v) Glycerol

$6 \%(\mathrm{w} / \mathrm{v})$ SDS

$0.02 \%(w / v)$ Bromophenol blue

add freshly $10 \%(\mathrm{v} / \mathrm{v}) \beta$-Mercaptoethanol to $2 x$ working solution

Before loading, the protein samples were supplemented with 2x SDS-sample buffer, denatured at $95^{\circ} \mathrm{C}$ for $5 \mathrm{~min}$ and subsequently centrifuged for $5 \mathrm{~min}$ at $13000 \times \mathrm{g}$. To estimate the size of the separated proteins, a protein ladder $(10 \mu$ of unstained or $4 \mu \mathrm{l}$ of pre-stained PageRuler Protein Ladder, from Thermo Scientific) was loaded alongside. The gel electrophoresis was performed in running buffer at $25 \mathrm{~mA}$ for the stacking gel and at $35 \mathrm{~mA}$ for the dissolving gel or overnight at $\sim 7 \mathrm{~mA}$. 


\subsubsection{Coomassie Staining}

SDS-polyacrylamide gels were stained with Coomassie Brilliant Blue to control the expression of recombinant proteins or to analyze in vitro binding studies.

Staining solution: $50 \%(\mathrm{v} / \mathrm{v})$ Methanol

$0.25 \%$ (w/v) Coomassie Brilliant Blue R250

(first dissolved in methanol)

$10 \%(\mathrm{v} / \mathrm{v})$ Glacial acetic acid

Destaining solution: $5 \%(\mathrm{v} / \mathrm{v})$ Methanol

$10 \%(v / v)$ Glacial acetic acid

Upon gel electrophoresis, the SDS-polyacrylamide gels were stained with staining solution for $10 \mathrm{~min}$. Afterwards, the gels were incubated with destaining solution, until protein bands were visible. To accelerate the destaining, a tissue was placed besides the gel and the complete approach was shortly heated in a microwave.

\subsubsection{Western blot analyses}

Upon protein separation by SDS-PAGE, the proteins were electrophoretically transferred to a nitrocellulose membrane to facilitate the immuno-detection with chemiluminescent substrates. The method was initially described by Towbin et al. (1979) and was performed with some modifications.

Blotting buffer: $\quad 25 \mathrm{mM}$ Tris-Base $\mathrm{pH} 8.3$

$192 \mathrm{mM}$ Glycine

$20 \%(\mathrm{v} / \mathrm{v})$ Methanol (added freshly)

Ponceau solution: $0.2 \%(w / v)$ Ponceau S

$5 \%(\mathrm{v} / \mathrm{v})$ Glacial acetic acid

TBST (pH 7.4): $\quad 50 \mathrm{mM}$ Tris-Base $\mathrm{pH} 7.4$

$150 \mathrm{mM} \mathrm{NaCl}$

$0.1 \%(v / v)$ Tween-20

For the transfer, a semi-dry electro blot system was used. The SDS-polyacrylamide gel, the nitrocellulose membrane with the size of the gel and two Whatman papers were pre-soaked in blotting buffer and subsequently, the following sandwich was set up in the blot apparatus (onto the anode): 
(1) one Whatman paper $(0.8 \mathrm{~mm})$

(2) nitrocellulose membrane (Amersham Protran $0.45 \mu \mathrm{m} \mathrm{NC)}$

(3) SDS-polyacrylamide gel

(4) one Whatman paper $(0.8 \mathrm{~mm})$

All air bubbles were removed, the cathode was installed and the electro blot was performed for $1.5 \mathrm{~h}$ at $1.5 \mathrm{~mA} / \mathrm{cm}^{2}$. To control the efficiency of the transfer, the membrane was subsequently stained with Ponceau solution for $\sim 5 \mathrm{~min}$ and destained with water, until protein bands were visible. If required, the membrane was cut and the now visible bands of the unstained protein ladder were marked.

To block unspecific protein binding sites, the membrane was incubated with agitation for $\sim 1 \mathrm{~h}$ in $5 \%(\mathrm{w} / \mathrm{v})$ milk powder in TBST. Afterwards, the desired primary antibody diluted in $2 \%(\mathrm{w} / \mathrm{v})$ milk powder in TBST was added and incubated overnight at $4{ }^{\circ} \mathrm{C}$ or for at least $2 \mathrm{~h}$ at RT. Upon three times washing with TBST, the secondary antibody coupled to the enzyme Horseradish Peroxidase (HRP) was diluted in $2 \%(\mathrm{w} / \mathrm{v})$ milk powder in TBST and added to the membrane for $2 \mathrm{~h}$ at RT. Subsequently, the membrane was washed twice in TBST and once in TBS. Then, detection was performed with the chemiluminescent substrate "Amersham ECL Prime Western Blotting Detection Reagent" (GE Healthcare) and the FUSION-SL chemiluminescence detection system (Peqlab).

\subsubsection{Quantification of western blot signals}

The intensity of chemiluminescent signals of Western blot analyses was quantified by using the Bio1D Software (Peqlab). In co-immunoprecipitation studies, the band of the pull-down was related to the band of the interaction partner. Subsequently, the ratio of the wild type was set to $100 \%$ and the ratios of mutant or treated strains were related to the wild type. The number of independent experiments that were quantified to calculate the mean values and the standard derivations are always indicated. The p-values were calculated with an unpaired Student's t-test (type 3) by using the Microsoft Excel software. 


\section{RESULTS}

\subsection{Analysis of the role of Dbp5 in the nuclear export of ribosomal particles}

\subsubsection{Mutants of DBP5 are defective in the nuclear export of pre-40S and pre-60S particles}

The DEAD-box RNA-helicase Dbp5/Rat8 is known for its roles in the nuclear mRNA export at the cytoplasmic side of the NPC and in translation termination in the cytoplasm. For its second function, Dbp5 is bound to polysomes and this polysomal association is detectable in sucrose-density fractionation experiments (Gross et al., 2007). To analyze the localization of Dbp5 also in the non-ribosomal, the free $40 \mathrm{~S}$ and 605 subunits and $80 \mathrm{~S}$ ribosomes containing fractions of sucrose-density gradients, these experiments were repeated with wild type yeast cells. For such assays, the cells were treated with cycloheximide to prevent the polysome run-off. The antibiotic cycloheximide inhibits the release of deacylated tRNAs from the ribosomal E-site, so that the $80 \mathrm{~S}$ ribosomes are stalled during elongation and stay bound to the mRNAs (Masek et al., 2011). After cell lysis, the lysates were loaded onto the top of linear sucrose-gradients and ultra-centrifuged to separate particles by its density. Subsequently, the gradients were fractionated while measuring the absorbance at $254 \mathrm{~nm}$ to follow the distribution of the ribosomal RNA. Thus, typical polysome profiles were generated as shown in Figure 17A. To analyze the associated proteins of each fraction, all proteins were precipitated with trichloroacetic acid and the entire protein content was separated by SDS-PAGE and analyzed by Western blotting. Some fractions were pooled to be able to load the complete gradient onto one SDS-polyacrylamide gel. 
A

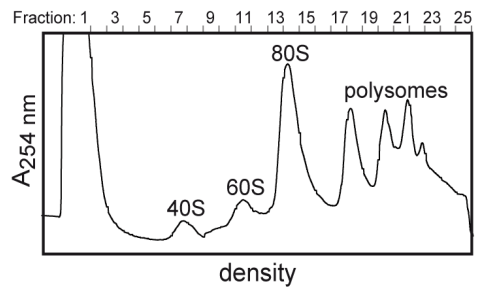

B

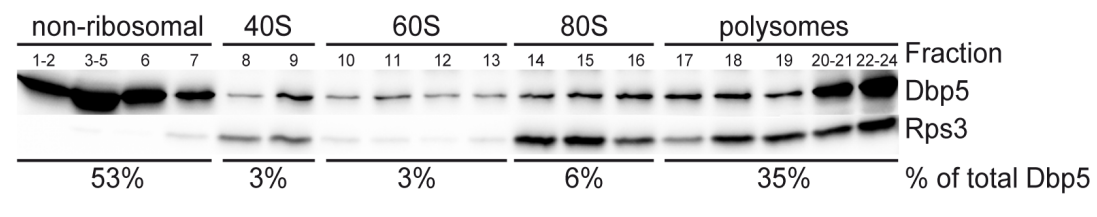

Figure 17: Dbp5 is present in fractions of both free ribosomal subunits.

(A) The polysome profile of wild type yeast cells, which were shifted for $1 \mathrm{~h}$ to $37^{\circ} \mathrm{C}$, is shown and the different ribosomal particles are indicated. Ten $\mathrm{OD}_{260 \mathrm{~nm}}$ units of the lysate were loaded onto a linear 7-47 \% sucrose gradient, ultra-centrifuged and fractionated while measuring the absorbance at $254 \mathrm{~nm}\left(\mathrm{~A}_{254 \mathrm{~nm}}\right)$. (B) The proteins of the corresponding fractions of $(A)$ were precipitated with trichloroacetic acid and analyzed by Western blotting with antibodies against Dbp5 and the small ribosomal protein Rps3. The ratios of total Dbp5 in the non-ribosomal, 40S, 60S, 80S and polysomal fractions are indicated.

As expected for its functions in mRNA export and translation termination, Dbp5 was particularly detected in the non-ribosomal and in the polysomal fractions of the sucrose-density gradient (Figure 17B). The small ribosomal protein Rps3 is part of the $40 \mathrm{~S}$ subunit and as such visible in the 40S, $80 \mathrm{~S}$ and polysomal ribosomes containing fractions (Figure 17B). The detection of Rps3 was always performed to confirm the localization of the different ribosomal particles in the sucrose-density gradient. Interestingly, around $3 \%$ of total Dbp5 was also associated with free $40 \mathrm{~S}$ and 60S subunits, respectively (Figure 17B) indicating that Dbp5 might have a function on the ribosomal subunits prior to translation termination.

Considering that Dbp5 is a well-known mRNA transport factor, the RNA-helicase might also be involved in the nuclear export of pre-ribosomal subunits. To test this hypothesis, fluorescence microscopy studies with GFP-tagged ribosomal proteins in different temperature-sensitive mutants of $D B P 5$ (see section 4.4.7) were performed. Enhanced GFP-tagged Rps2 served as a well-established reporter for the small (Milkereit et al., 2003) and Rpl25-GFP as a marker for the large ribosomal subunit (Gadal et al., 2001). Additionally, the export factor Nmd3-GFP, which is the Xpo1 adapter protein for the large pre-ribosomal subunit and binds in the nucleoplasm before export, was localized as a marker for the pre-60S particles (Gadal et al., 2001). All tagged reporter genes were expressed from plasmids under their own promoters to analyze the ribosomal export in the different yeast strains. 
A
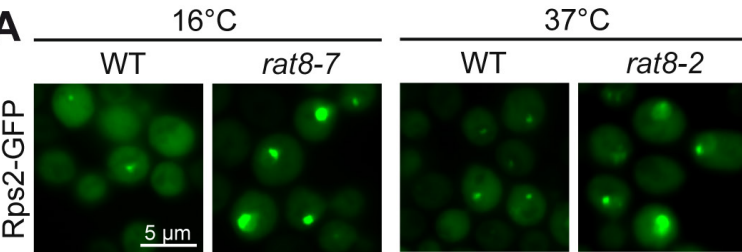

B

WT $16^{\circ} \mathrm{C}$
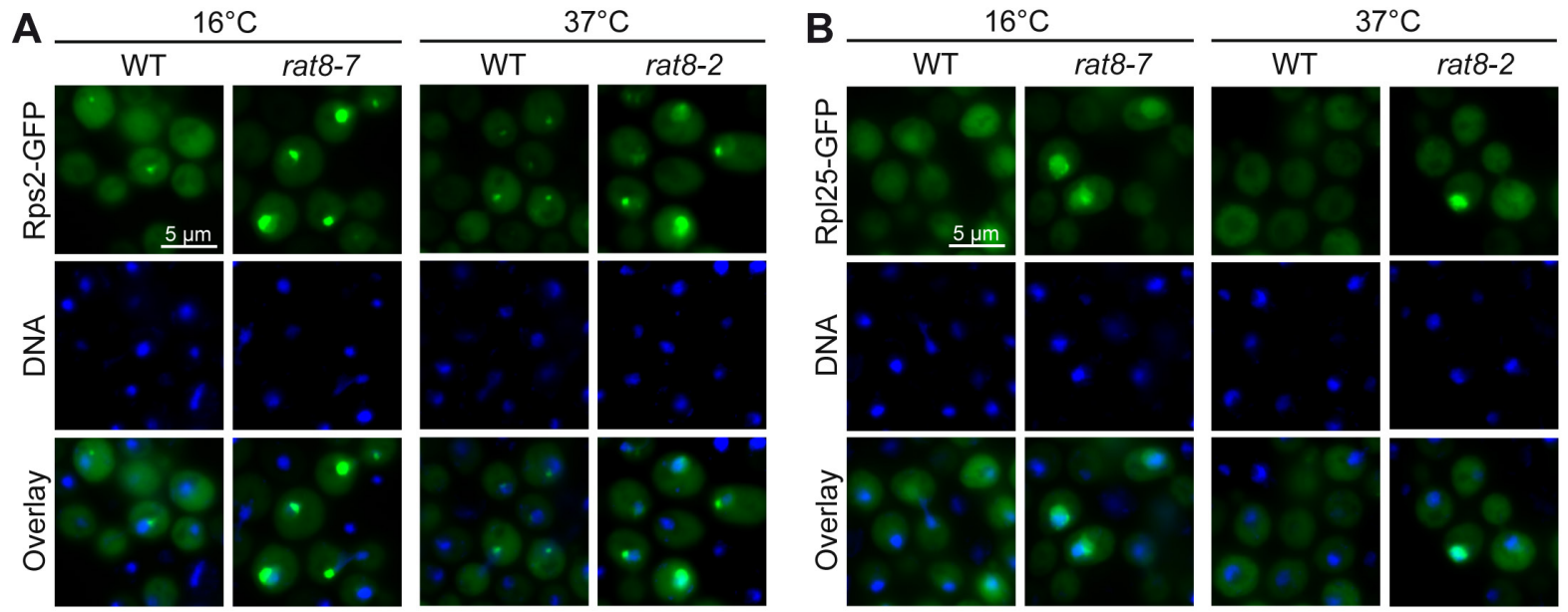

C

$37^{\circ} \mathrm{C}$
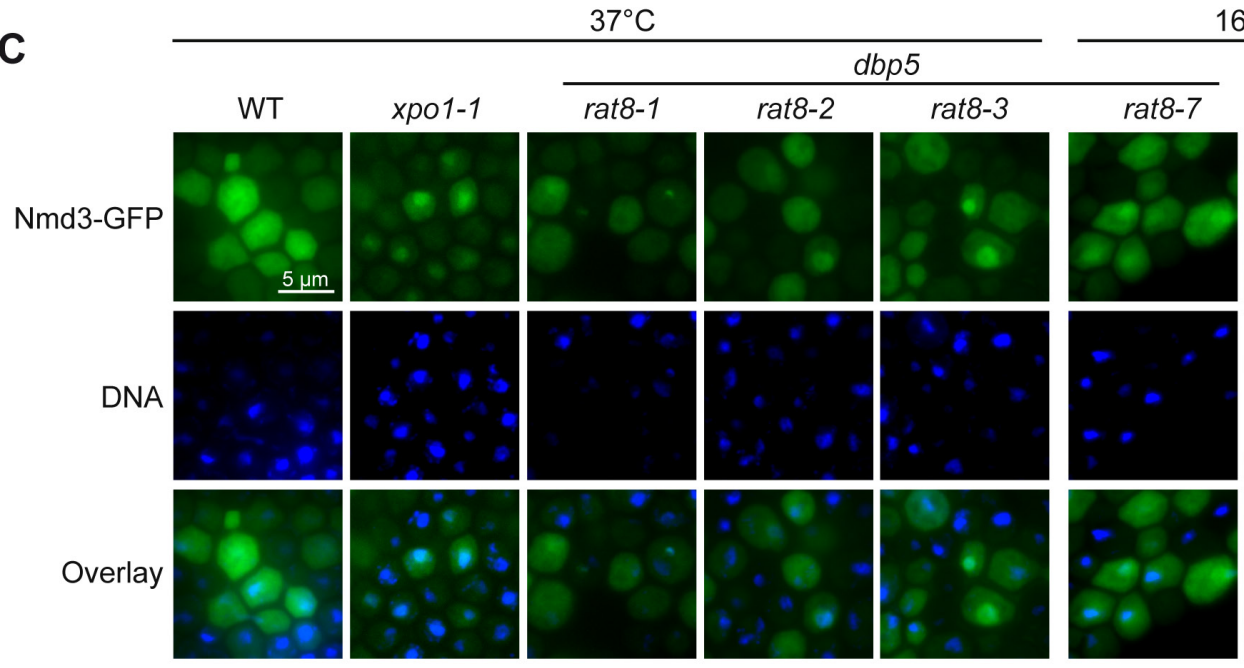

WT
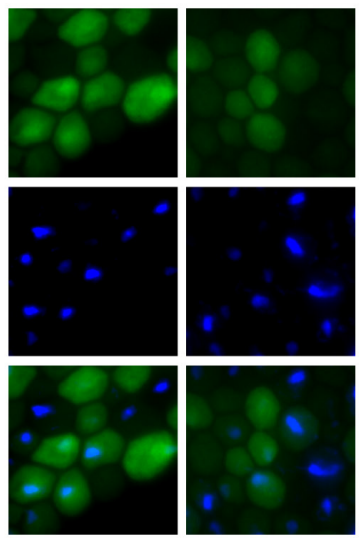

Figure 18: Both pre-ribosomal subunits accumulate in the nuclei of different $d b p 5$ mutants. (A-B) Fluorescence microscopy pictures localize Rps2-GFP as reporter for the small ribosomal subunit (A) and Rpl25-GFP a marker for the large ribosomal subunit (B) to the nuclei of the $d b p 5$ mutants rat8-7 and rat8-2 upon $1 \mathrm{~h}$ shift to their indicated non-permissive temperatures. The DNA was stained with Hoechst. (C) Fluorescence microscopy studies show that the pre-60S transport factor Nmd3-GFP accumulates in the nuclei of different $d b p 5$ mutants after shift for $1 \mathrm{~h}$ to their indicated restrictive temperatures. The mutant of the karyopherin Xpo1/Crm1, xpo1-1, serves as a positive control. The DNA was stained with Hoechst. WT = wild type

At $37^{\circ} \mathrm{C}$ and $16^{\circ} \mathrm{C}$, wild type cells showed the expected equal nuclear and cytoplasmic distribution of Rps2-GFP, but also in several cells a single dot beside the stained DNA (Figure 18A). These dots might represent the nucleolus, where the ribosomal proteins are incorporated into the ribosomal particles. However, the coldsensitive $d b p 5$ mutant rat8-7 as well as the temperature-sensitive mutant rat8-2 additionally accumulated Rps2-GFP in the nucleoplasm upon shift to their restrictive temperatures (Figure 18A) indicating defects in the nuclear export of pre-40S subunits. Similarly, the 60S reporter Rpl25-GFP was mislocalized to the nucleus of temperature shifted rat8-7 and rat8-2 cells (Figure 18B). Indeed, even some wild type cells showed an increased nuclear staining of Rpl25-GFP at $16^{\circ} \mathrm{C}$ (Figure 18B) that might be caused by the low temperature. Possibly, the transport of the pre-60S 
subunits is generally decelerated at $16^{\circ} \mathrm{C}$ leading to an extended localization in the nucleoplasm, because this effect was also visible for the pre-60S adapter Nmd3-GFP in wild type cells at $16^{\circ} \mathrm{C}$ (Figure 18C). However, the nuclear signal of both $60 \mathrm{~S}$ reporters was clearly enhanced in the rat8-7 mutant (Figure 18B+C) suggesting that also the nuclear export of large pre-ribosomal subunits is defective in the $d b p 5$ mutants. Furthermore, Nmd3-GFP accumulated to different extents in the nuclei of the temperature-sensitive $d b p 5$ mutants rat8-1, rat8-2 and rat8-3, whereby rat8-1 cells were only marginally affected at $37^{\circ} \mathrm{C}$ (Figure 18C). The xpo1-1 mutant of the karyopherin Xpo1/Crm1 served as a positive control for cells with ribosomal export defects and showed the expected increased nuclear staining of Nmd3-GFP at $37^{\circ} \mathrm{C}$ (Figure 18C). In summary, these experiments suggest that $d b p 5$ mutants have defects in the transport of both, pre-60S and pre-40S subunits.

In support of these data, similar results were obtained from fluorescence in situ hybridization (FISH) experiments with probes against the $18 \mathrm{~S}$ and $25 \mathrm{~S}$ rRNAs, which were performed by Haijia Wu (laboratory of Prof. Heike Krebber) with the different temperature-sensitive dbp5 mutants (data not shown). The probes recognize the mature rRNAs as well as their precursor molecules. Additionally, Alexandra Hackmann (laboratory of Prof. Heike Krebber) analyzed the known mRNA export defects in the dbp5 mutants by localization of poly(A)-tail containing RNAs with Cy3labeled oligo(dT)-probes (data not shown). The statistical analysis of these FISH experiments is shown in Figure 19. 


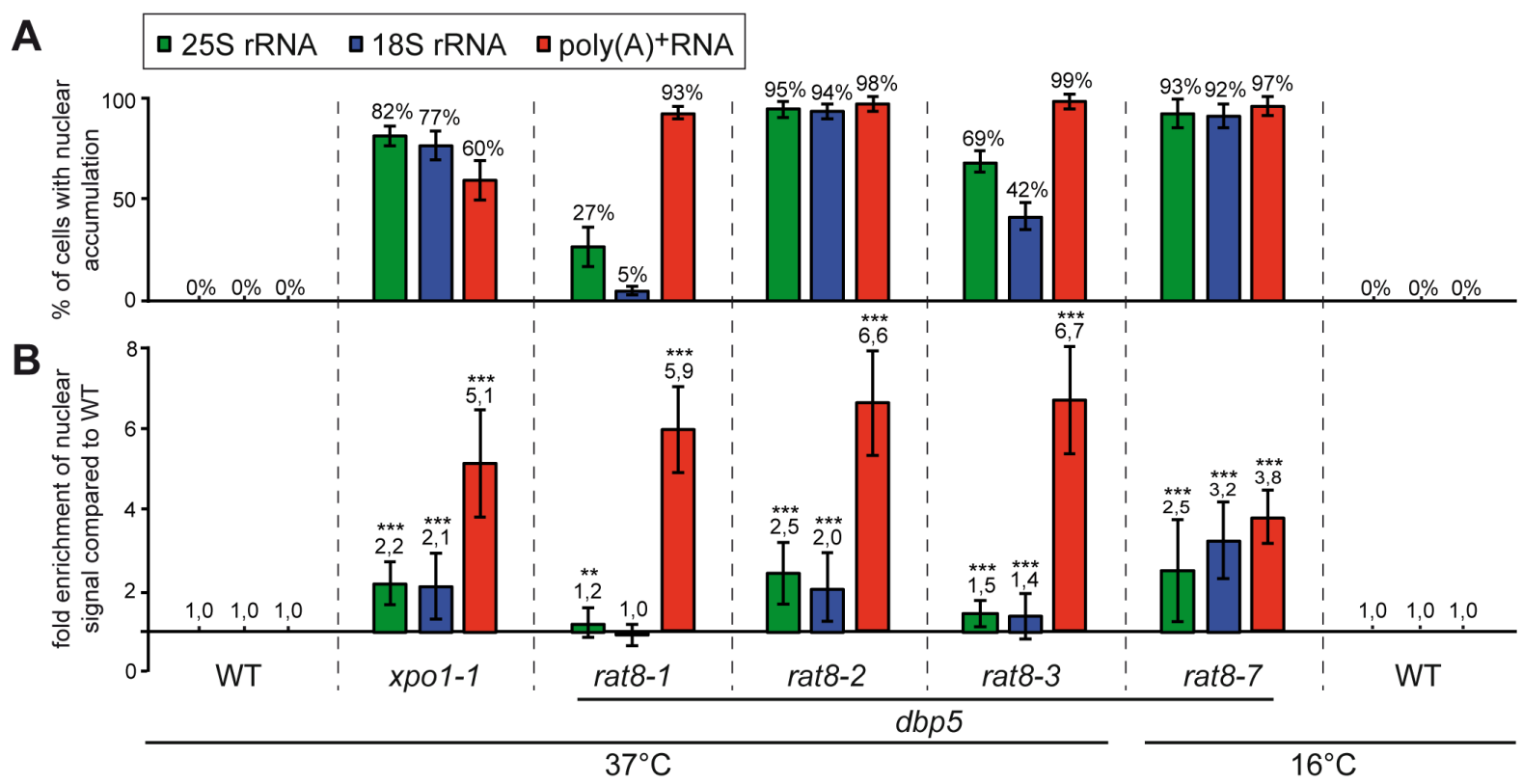

Figure 19: The ribosomal export defects do not correlate with the mRNA export defects in the dbp5 mutants.

$(A+B)$ The statistical analysis of FISH experiments is shown, which were performed with probes against the 25S rRNA, 18S rRNA and poly(A) ${ }^{+}$RNA in xpo1-1 and different dbp5 mutants upon $1 \mathrm{~h}$ shift to their indicated restrictive temperatures (performed by Haijia Wu and Alexandra Hackmann, laboratory of Prof. Heike Krebber). (A) The ratio of cells that accumulate the fluorescent signals in the nucleus is indicated for each strain. (B) The diagram shows the average enrichment of the nuclear signal for each mutant. The nuclear signal of at least ten cells with phenotype was quantified per strain and related to the fluorescent signal of the whole cell and to the ratio of the wild type. Error bars represent the standard deviation and $\mathrm{p}$-values were calculated by an unpaired Student's t-test (type 2). ${ }^{\star \star \star}=p<0.001,{ }^{\star \star}=p<0.01$, WT $=$ wild type

More than $90 \%$ of the rat8-2 and rat8-7 cells revealed a nuclear accumulation of both, the $18 \mathrm{~S}$ and the 25S rRNAs upon temperature shifts (Figure 19A). Furthermore, the nuclear signal was on average more than twofold increased relative to the whole cell and the wild type and hence, was comparable to the enrichment in the established export receptor mutant xpo1-1 (Figure 19B). The nuclear mislocalization of the $18 \mathrm{~S}$ and $25 \mathrm{~S}$ rRNAs was also visible in the dbp5 mutant rat8-3, but the effect was less strong and affects fewer cells (Figure 19). The weakest defects were seen in the strain rat8-1, in which only $27 \%$ of the cells accumulated the 25S rRNAs and around $5 \%$ of the cells mislocalized the 18S rRNAs in their nuclei (Figure 19A). Thus, these slight accumulations were hardly detectable as enrichment in the quantifications (Figure 19B). In summary, the observed nuclear accumulations of small and large ribosomal proteins and rRNAs in the dbp5 mutants indicate that the nuclear export of both pre-ribosomal subunits is disturbed in these cells, even though the strength differs in each mutant. Thus, Dbp5 is required for efficient ribosomal transport. 
Remarkably, the intensities of the defects in ribosomal transport do not correlate with the mRNA export defects in the different $d b p 5$ mutants (Figure 19). In particular, more than $90 \%$ of the rat8-7 cells revealed a comparatively strong nuclear enrichment of $18 \mathrm{~S}$ rRNAs (3.2 fold) and of 25S rRNAs (2.5 fold). Nearly all rat8-7 cells also accumulated mRNAs in the nucleoplasm, but the enrichment of the nuclear signal was modest ( $\sim 3.8$ fold) compared to the other $d b p 5$ mutants ( $>5$ fold) indicating a rather mild mRNA export defect. Vice versa, rat8-1 and rat8-3 cells showed only slight ribosomal mislocalizations, whereas their mRNA export was strongly impaired. In contrast, rat8-2 cells strongly accumulate both, mRNAs and ribosomal particles in their nuclei. Thus, the missing correlation between mRNA and ribosomal transport defects in all $d b p 5$ mutants suggests that the observed ribosomal mislocalizations are not only caused by the defects in nuclear mRNA export.

\subsubsection{The biogenesis of ribosomal subunits is not obviously affected in the dbp5 mutants}

However, the biogenesis of ribosomal subunits could also be defective in the $d b p 5$ mutants leading to disturbed transport and to the nuclear accumulation of ribosomal particles (Tschochner and Hurt, 2003). Thus, the steady state level of the different rRNAs and their precursor molecules was analyzed by Northern blotting in the strongest ribosomal transport mutants rat8-2 and rat8-7 to detect potential defects in the rRNA processing. Therefore, the total RNA of the temperature shifted $d b p 5$ mutants was extracted and separated on formaldehyde containing $1 \%$ agarose/MOPS gels to eliminate secondary structures. Furthermore, the temperature-sensitive mutants rat7-1/nup159 and xpo1-1, which are known to have defects in the transport of both pre-ribosomal particles upon temperature shifts (Moy and Silver, 1999, 2002; Stage-Zimmermann et al., 2000), were analyzed as control. The different rRNA species were detected by non-radioactive Northern blotting using DIG-labeled RNA probes. As the mature $18 \mathrm{~S}$ and $25 \mathrm{~S}$ rRNAs arise from one precursor molecule by a series of cleavage events (Figure 1B), the specific probes can always detect the precursor molecules as well (Figure 20). 


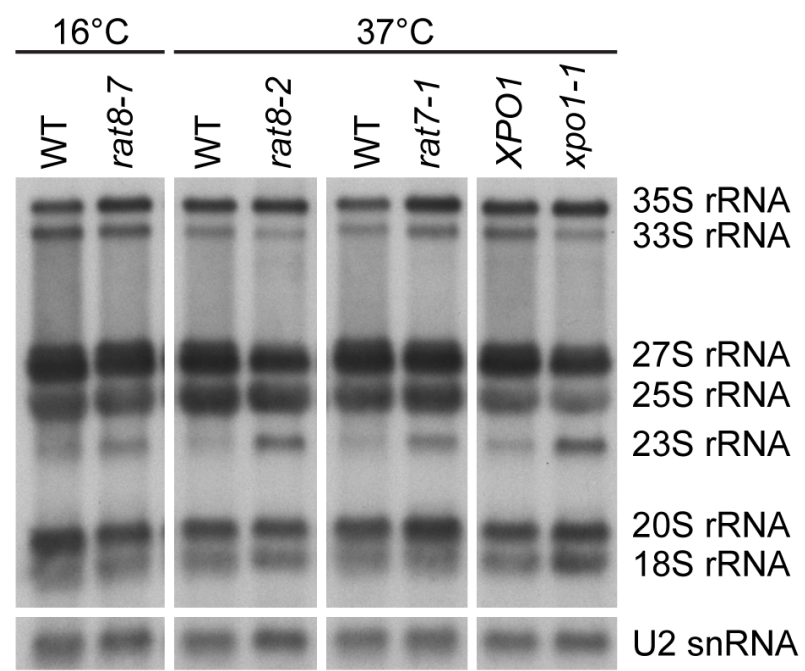

Figure 20: The steady state level of the different rRNA species in dbp5 mutants is comparable to other ribosomal export factor mutants.

A Northern blot analysis is shown upon gel electrophoresis (1\% agarose/MOPS/formaldehyde gel) of $1 \mu \mathrm{g}$ total RNA extracted from the different strains upon $1 \mathrm{~h}$ shift to the indicated restrictive temperatures. Non-radioactive detection was performed with DIG-labeled RNA probes against 27S, 25S, $20 \mathrm{~S}$ and $18 \mathrm{~S}$ rRNAs, which also recognize their precursor molecules. The detection of the U2 snRNA served as loading control. A xpo1 $\triangle$ strain with a XPO1 or xpo1-1 containing plasmid was used as control. WT = wild type

Only minor changes in the steady state level of most of the different rRNA species were visible: the amount of $35 \mathrm{~S}$ pre-rRNA was slightly increased in all export mutants, whereas the level of the 33S pre-rRNA decreased in rat8-2 as well as xpo1-1 and increased in rat7-1 cells (Figure 20). Furthermore, the amount of the $27 \mathrm{~S}$ pre-rRNAs appeared slightly reduced in rat8-2 and xpo1-1, whereas the steady state levels of the mature rRNAs and of the 20S pre-rRNAs were not greatly altered in the different mutants (Figure 20). The most notable change was that in all export mutants an additional rRNA species appeared: the aberrant 23S rRNA, which derives from $35 \mathrm{~S}$ processing defects with a premature $A_{3}$ cleavage prior to splitting at the sites $A_{0}$, $A_{1}$ and $A_{2}$ (Figure 1B) (Venema and Tollervey, 1999). These results show that indeed some changes in the steady state rRNA levels are detectable in the dbp5 mutants rat8-2 and rat8-7, but such variations are also visible in the established ribosomal export mutants rat7-1 and xpo1-1. Thus, slight defects in the rRNA processing could also be a typical phenotype of ribosomal export mutants and might be caused indirectly by the nuclear accumulation of pre-ribosomal particles.

To analyze whether the production of ribosomal subunits is generally disturbed in the dbp5 mutants, their total amount of ribosomal subunits was examined. Therefore, density gradient centrifugation was performed with yeast lysates treated with $100 \mathrm{mM}$ EDTA, which forms chelate complexes with $\mathrm{Mg}^{2+}$ ions. The consequent lack of $\mathrm{Mg}^{2+}$ ions leads to the splitting of all $80 \mathrm{~S}$ ribosomes into free $40 \mathrm{~S}$ and $60 \mathrm{~S}$ subunits that 
are represented by two distinct peaks in the ribosomal profiles (Figure 21) (Ho and Johnson, 1999). These profiles follow the RNA by measuring the absorbance at $254 \mathrm{~nm}$ and the peak sizes characterize the total cellular amount of the ribosomal subunits. To calculate the ratio between $60 \mathrm{~S}$ and $40 \mathrm{~S}$ subunits, the areas underneath their peaks were quantified and set into relation. Wild type cells should have a $60 S: 40 S$ ratio of 2.1:1, because the large ribosomal subunits contain twofold more rRNA than the small (Moy and Silver, 2002).

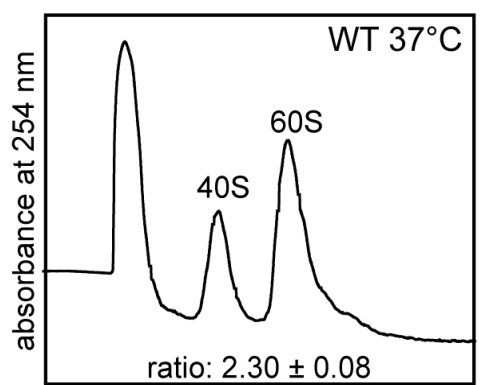

density

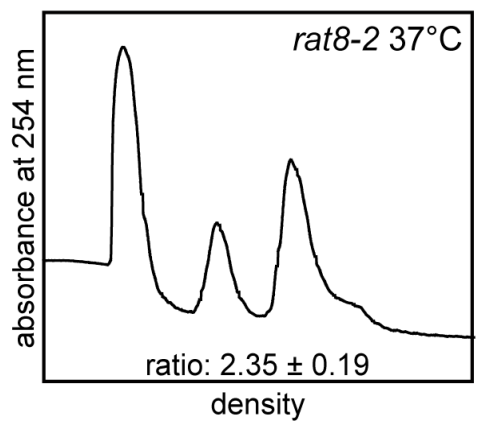

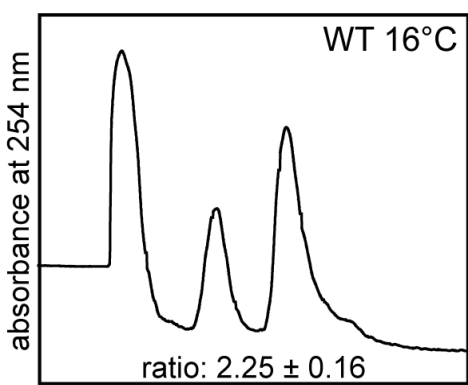

ratio: $2.25 \pm 0.16$

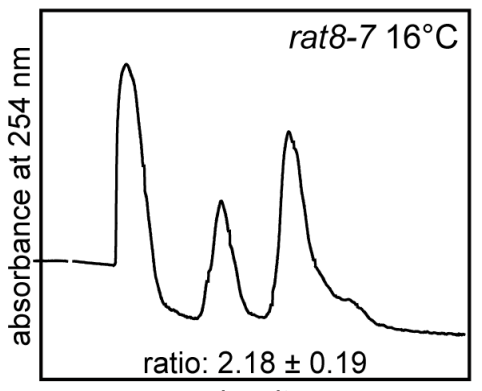

density

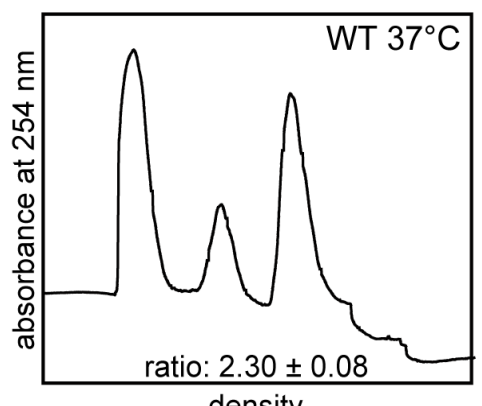

density

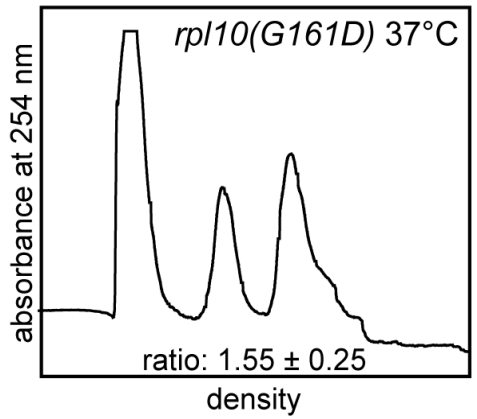

Figure 21: The total amount of ribosomal subunits is unchanged in the dbp5 mutants.

Ribosomal profiles are shown upon $1 \mathrm{~h}$ shift to the indicated restrictive temperatures and treatment of the lysates with $100 \mathrm{mM}$ EDTA for $20 \mathrm{~min}$ on ice. The average ratios of the area of the 60S to $40 S$ peak of at least three independent sucrose-gradient fractionation experiments are indicated. Errors represent the standard deviation. The strain rpl10(G161D) shows 60S biogenesis defects and served as a positive control of the assay. WT = wild type

Indeed, wild type cells showed independently of the temperature shifts the expected $60 S: 40 S$ ratio of $\sim 2.3$ (Figure 21). Similarly, the ratios of the dbp5 mutants rat8-2 and rat8-7 were not significantly changed compared to the wild type. Even the sizes of the 605 and the $40 S$ peaks were not reduced showing that the amount of both ribosomal subunits is not altered in rat8-2 and rat8-7, the dbp5 mutants with the strongest ribosomal export defects (Figure 19). In contrast, the size of the 60S peak of $r p / 10(G 161 D)$ is decreased and also its 60S:40S ratio is reduced to an average of 1.55 (Figure 21). This mutant of RPL10 has known defects in the cytoplasmic biogenesis of the large ribosomal subunit (Hedges et al., 2005) and its profile shows that maturation defects can indeed be visualized by this method.

Thus, these results indicate that the overall production of the ribosomal subunits is 
not substantially disturbed in the $d b p 5$ mutants. Therefore, it is unlikely that slight defects in the biogenesis of ribosomal subunits leads to the strong ribosomal mislocalizations detected by FISH and GFP-microscopy experiments.

\subsubsection{Dbp5 genetically and physically interacts with established ribosomal transport factors}

To further analyze whether Dbp5 is directly involved in ribosomal transport, genetic analyses were performed with established ribosomal export factors. The temperature-sensitive yeast strains $n m d 3-2$ and $m$ tr2-33 are known to disturb the nuclear export of the large pre-ribosomal subunit, but do not affect the mRNA transport upon shift to $37^{\circ} \mathrm{C}$ (Bassler et al., 2001; Gadal et al., 2001). Thus, these mutations were combined with the dbp5 mutants rat8-2 as well as rat8-3 (for yeast strain generation see section 5.8) and the resulting strains were spotted in serial dilutions onto selecting agar plates for growth analyses. All mutant strains were transformed with an additional plasmid that contains an URA3 selection marker and the corresponding wild typical gene. Thus, their growth defects are rescued on selective media. In contrast, plates with 5-Fluoroorotic Acid (FOA) select for cells that have lost the URA3-containing plasmid and thus, growth defects can be analyzed.

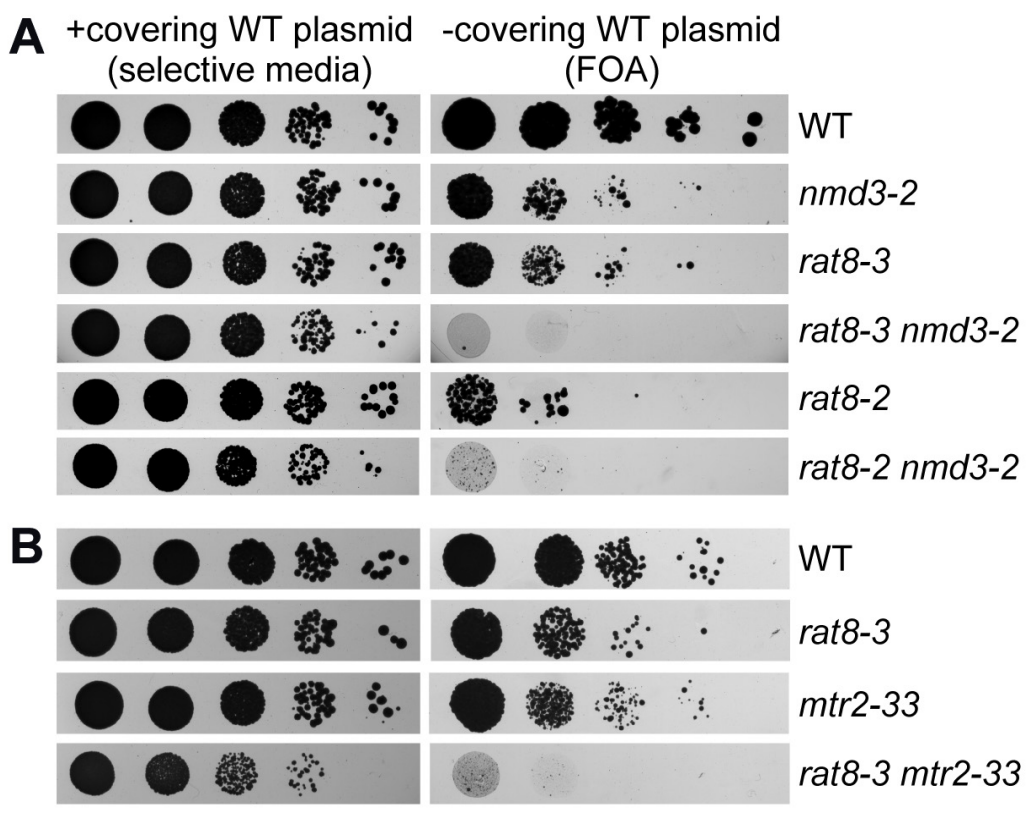

Figure 22: Dbp5 genetically interacts with the ribosomal transport factors $\mathrm{Nmd} 3$ and Mtr2. (A) Growth analyses of nmd3-2 combined with rat8-3 or rat8-2 show strong growth defects compared to the single mutants and the wild type (WT) after three days at $30^{\circ} \mathrm{C}$. The cells were spotted in serial dilutions onto agar plates either selecting for a covering wild typical plasmid or selecting for the loss of this plasmid (FOA = 5-Fluoroorotic Acid).

(B) The combination of rat8-3 and $n m d 3-2$ results in synthetic lethality compared to the single mutants and the wild type after three days at $30^{\circ} \mathrm{C}$. The growth analysis was performed as described in (A). 
The combination of rat8-3 with $n m d 3-2$ (Figure 22A) and with mtr2-33 (Figure 22B) resulted in a strong growth defect compared to the single mutants and the wild type at the semi-permissive temperature of $30^{\circ} \mathrm{C}$. The same was visible for the combination of rat8-2 with $n m d 3-2$ (Figure 22A). Such synthetic lethality argues for a function of both genes in the same pathway. Since mtr2-33 and nmd3-2 are specifically defective in the transport of ribosomal particles, their synthetic lethality with Dbp5 suggests a role of this RNA-helicase in this process as well.

If Dbp5 directly transports pre-ribosomal subunits, a physical contact of Dbp5 to the exported particles and bound transport factors would be expected. To test this hypothesis, co-immunoprecipitation experiments were performed to analyze the protein interactions in vivo. Wild type cells transformed with plasmids containing XPO1-GFP or NMD3-GFP as well as a genomically tagged DBP5-GFP strain transformed with a NMD3-13XMYC expressing plasmid were used. The GFP-tagged proteins were immunoprecipitated and their interacting proteins were analyzed by subsequent SDS-PAGE and Western blotting.
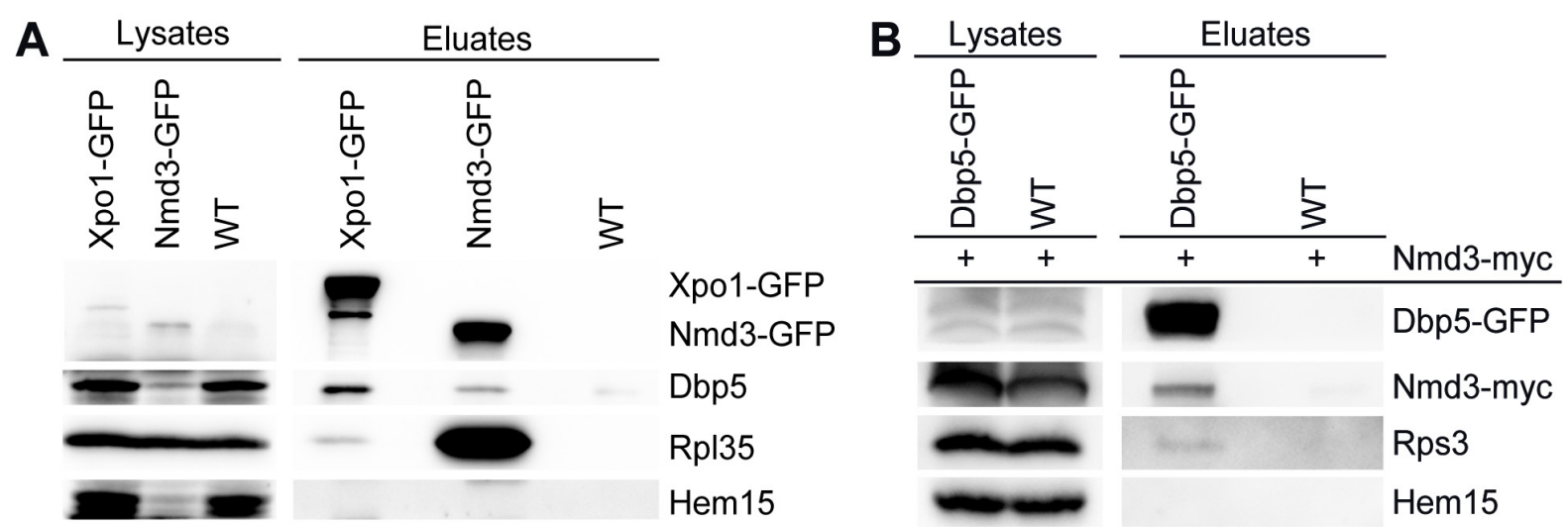

Figure 23: Dbp5 physically interacts with the ribosomal export factors Xpo1 and Nmd3.

(A) Western blot analysis of an immunoprecipitation experiment shows co-precipitation of Dbp5 and the ribosomal protein Rpl35 in the Xpo1-GFP and Nmd3-GFP pull-downs upon RNase A treatment. (B) The Western blot analysis reveals co-precipitation of the ribosomal protein Rps3 and plasmid borne Nmd3-myc in the Dbp5-GFP pull-down. (A+B) The tagged proteins were detected with antibodies against their tags, all other proteins with specific antibodies. Wild type (WT) cells without GFP-tagged proteins and detection of Hem15 (Ferrochelatase) served as non-binding controls.

Dbp5 was co-precipitated in the pull-downs of Xpo1-GFP and Nmd3-GFP (Figure 23A) and vice versa, Nmd3-myc was detectable in the immunoprecipitation of Dbp5GFP (Figure 23B) indicating that Dbp5 is associated with Xpo1- and Nmd3containing particles. Immunoprecipitation experiments with no GFP-tagged proteins containing wild type cells demonstrate that the co-precipitated proteins do not equally bind unspecific to the GFP-trap agarose beads (Figure 23). Furthermore, the 
approach shown in Figure 23A was treated with RNase A to degrade single-stranded RNAs. As Dbp5 is still detectable in the Xpo1-GFP and Nmd3-GFP immuneprecipitations, these proteins must interact via protein-protein contact or via protected RNAs such as in the ribosomal particles. However, whether Dbp5 directly binds to Xpo1 or Nmd3 cannot be answered from such in vivo experiments. Nevertheless, these results show that Dbp5 is able to associate with the exported pre-ribosomal subunits.

In summary, the genetic and physical interactions of Dbp5 with established ribosomal transport factors suggest that Dbp5 is directly involved in the nuclear export of preribosomal subunits.

\subsubsection{Dbp5 rather acts at the cytoplasmic side of the NPC than on ribosomal particles during translocation through the NPC}

If Dbp5 is required for ribosomal transport, the question is where it is needed during this process. One possibility is that Dbp5 accompanies the ribosomal particles from the nucleus into the cytoplasm like other export factors such as Nmd3, Xpo1 or Mtr2 (Gerhardy et al., 2014). If so, Dbp5 should accumulate together with the preribosomal subunits in the nuclei of mutants defective in ribosomal transport. However, for its function in mRNA export, Dbp5 is positioned at the cytoplasmic fibrils of the NPC and remodels emerging mRNPs (Hodge et al., 1999; Lund and Guthrie, 2005; Schmitt et al., 1999; Tran et al., 2007; Weirich et al., 2004). Therefore, Dbp5 might also act at the cytoplasmic side of the NPC during ribosomal transport. To distinguish between these possibilities, the localization of Dbp5-GFP was analyzed in nmd3-2, mtr2-33 and xpo1-1 cells that accumulate pre-ribosomal subunits in their nuclei upon shift to $37^{\circ} \mathrm{C}$ (Figure 21) (Bassler et al., 2001; Gadal et al., 2001; Moy and Silver, 1999; Stage-Zimmermann et al., 2000). The strains were transformed with DBP5-GFP containing plasmids and fluorescence microscopy was performed. 


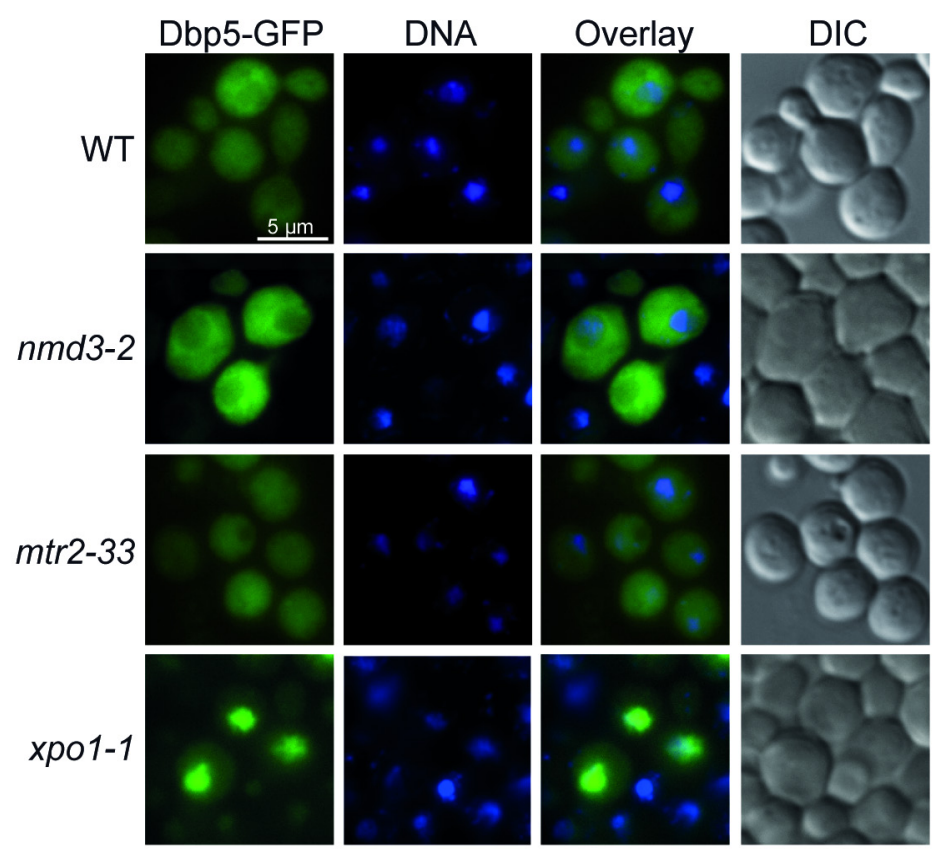

Figure 24: Dbp5 is not transferred together with the ribosomal particles into the cytoplasm. Fluorescence microscopy pictures reveal a mostly cytoplasmic localization of plasmid borne Dbp5GFP in wild type (WT), nmd3-2 and mtr2-33 cells, whereas Dbp5-GFP accumulates in the nuclei of $x$ po1-1 cells upon $1 \mathrm{~h}$ shift to $37^{\circ} \mathrm{C}$. The DNA was stained with Hoechst. DIC $=$ differential interference contrast

In wild type cells, Dbp5-GFP showed a mainly cytoplasmic distribution that was not altered in the mutants $n m d 3-2$ and mtr2-33 upon shift to their restrictive temperatures (Figure 24). These results indicate that Dbp5 is not transferred together with the preribosomal particles from the nucleus into the cytoplasm. In contrast, Dbp5-GFP accumulated in the nuclei of temperature shifted xpo1-1 cells (Figure 24). Xpo1 is a karyopherin, which is generally involved in nuclear protein export (Stade et al., 1997). Therefore, it is more likely that free Dbp5, which is not attached to ribosomal particles, is actively exported from the nucleus by Xpo1, as suggested previously by Hodge et al. (1999). In conclusion, these results indicate that Dbp5 does not accompany ribosomal particles through the NPCs.

Thus, it remains possible that Dbp5 acts on the pre-ribosomal subunits at the cytoplasmic side of the NPC, as for nuclear mRNA export. Localized to the cytoplasmic fibrils, Dbp5 might shortly contact the emerging ribosomal particles or might be loaded onto them and might stay associated during the cytoplasmic biogenesis or even until translation. To clarify whether Dbp5 is bound to the preribosomal subunits during maturation, its interaction with other ribosomal export factors was analyzed in the temperature-sensitive strain drg1-18. The AAA-ATPase Drg1 catalyzes the first cytoplasmic maturation step of the large pre-ribosomal subunit, which is a prerequisite for the following steps (Figure 4). In the drg1-18 
mutant, the cytoplasmic biogenesis is interrupted and aberrant pre-60 particles bound to shuttling export and biogenesis factors such as Arx1 or Nmd3 accumulate in the cytoplasm (Kappel et al., 2012; Pertschy et al., 2007). If Dbp5 is loaded onto pre-ribosomal subunits at the NPC and is associated during their cytoplasmic biogenesis steps, an increased binding between Dbp5 and Nmd3 is expected in drg1-18 cells. To test this, co-immunoprecipitation studies were conducted with wild type and drg1-18 cells transformed with GFP-DBP5 and NMD3-13XMYC containing plasmids. Additionally, strains carrying ARX1-GFP expressing plasmids were used as positive controls.
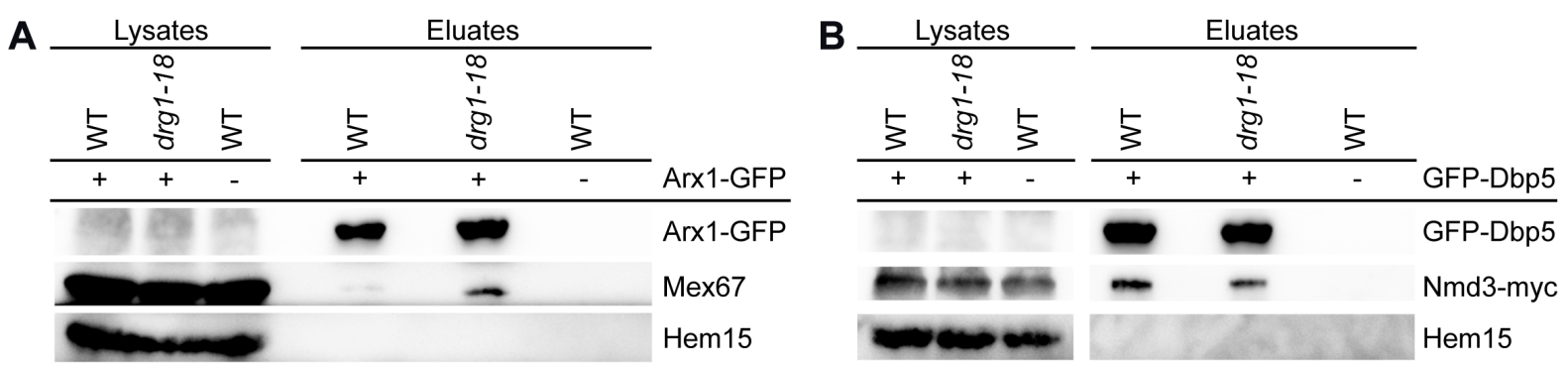

Figure 25: Dbp5 does not accumulate with $\mathrm{Nmd3}$ on immature cytoplasmic ribosomal particles in drg1-18 cells.

(A) An Arx1-GFP immunoprecipitation experiment shows increased co-precipitation of Mex67 in drg1-18 compared to wild type (WT) upon shift for $1 \mathrm{~h}$ to $37^{\circ} \mathrm{C}$ in the Western blot analysis. (B) Western blot analysis of a GFP-Dbp5 immunoprecipitation reveals equal amounts of coprecipitated Nmd3-myc in wild type and drg1-18 cells upon $1 \mathrm{~h}$ shift to $37^{\circ} \mathrm{C}$. All strains contain a NMD3-myc expressing plasmid. (A+B) Tagged proteins were expressed from plasmids and detected by antibodies against their tags. Wild type cells without GFP-tagged protein and detection of Hem15 (Ferrochelatase) served as negative controls.

As seen in Figure 25A, the binding of Arx1-GFP to Mex67-containing particles was enhanced in drg1-18 cells compared to wild type cells. The increased interaction between Arx1 and Mex67 confirms that both export factors indeed accumulate on the pre-60S subunits in this mutant, as shown earlier by Kappel et al. (2012). In contrast, the interaction between Dbp5 and $\mathrm{Nmd3}$ is not increased in the drg1-18 mutant (Figure 25B) suggesting that Dbp5 does not accumulate on the Nmd3-containg pre$60 \mathrm{~S}$ subunits in these cells. In this case, Nmd3, which is normally released following the Drg1 recycling step (Lo et al., 2010), represents the increasing amount of immature pre-60S particles bound to export factors. Thus, loading of Dbp5 onto the pre-ribosomal subunits at the cytoplasmic side of the NPC and binding during their cytoplasmic maturation seems to be unlikely and Dbp5 might rather act from the cytoplasmic filaments on the ribosomal particles prior to the Drg1 recycling step.

If the binding of Dbp5 to the cytoplasmic fibrils is indeed necessary for ribosomal transport, the lack of Dbp5 at the NPC should also result in ribosomal export defects. 
The nucleoporin Nup159/Rat7 is known to anchor Dbp5 to the NPC and Dbp5 is lost from the nuclear rim in the temperature-sensitive rat7-1 mutant at $37^{\circ} \mathrm{C}$ (Hodge et al., 1999). Therefore, this strain was analyzed regarding ribosomal export defects by performing fluorescence microscopy with the GFP-tagged reporter proteins.

A
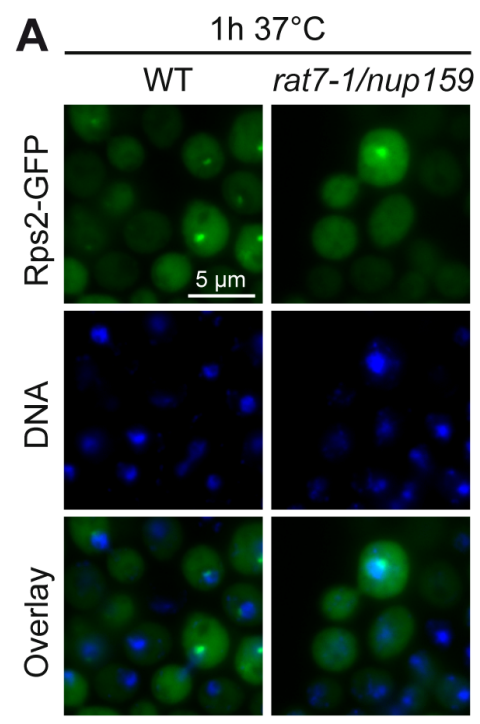

B

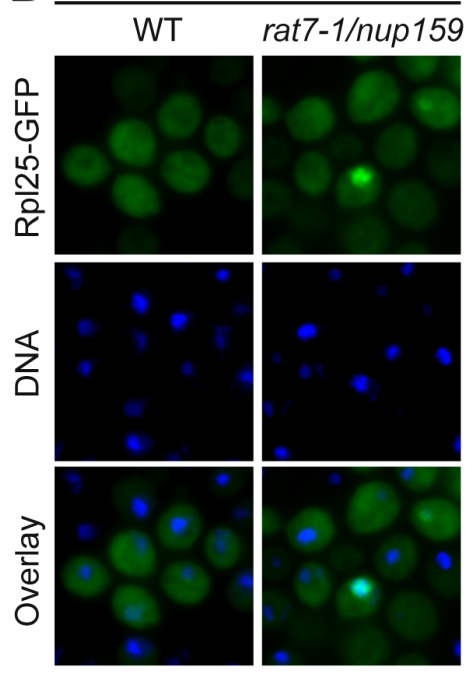

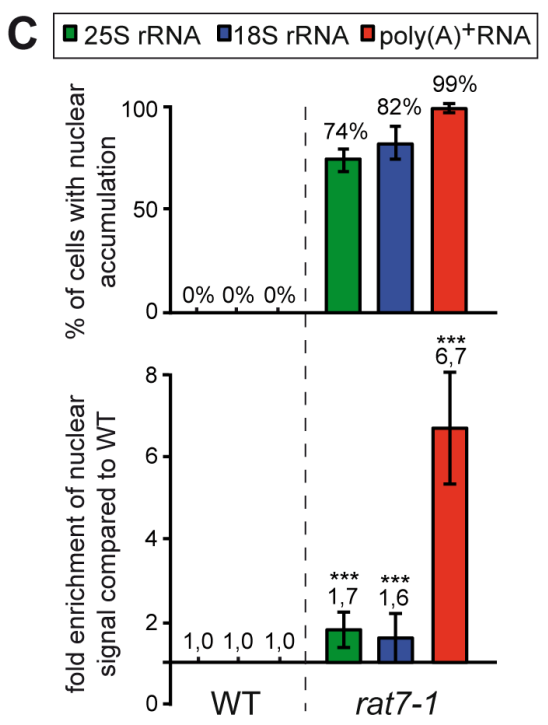

Figure 26: Slight nuclear accumulation of ribosomal particles is visible in rat7-1 cells.

(A+B) Fluorescence microscopy shows the nuclear accumulation of the $40 \mathrm{~S}$ reporter, Rps2-GFP (A) and the 60S reporter, Rpl25-GFP (B) in some rat7-1/nup159 cells upon $1 \mathrm{~h}$ shift to $37^{\circ} \mathrm{C}$. The DNA was stained with Hoechst. (C) Statistical analyses of FISH experiments that were performed by Haijia Wu and Alexandra Hackmann (laboratory of Prof. Krebber) as described in Figure 19.

Rps2-GFP and Rpl25-GFP accumulate in the nucleoplasm of several rat7-1 cells upon temperature shift to $37^{\circ} \mathrm{C}$ (Figure 26A+B). Similarly, FISH experiments performed by Haijia Wu and Alexandra Hackmann (laboratory of Prof. Krebber) revealed a slight, but significant nuclear accumulation of the $25 S$ and $18 \mathrm{~S}$ rRNAs in $74 \%$ and $82 \%$ of the rat7-1 cells, respectively (Figure $26 \mathrm{C}$ ). These data indicate that rat7-1 cells are defective in the transport of both pre-ribosomal subunits as well, but the effect is less strong than in the $d b p 5$ mutants rat8-2 and rat8-7 (Figure 19). In contrast, the mRNA export of rat7-1 cells was strongly impaired at $37^{\circ} \mathrm{C}$ with an on average 6.7 fold enriched nuclear signal in nearly all cells (Figure 26C). Thus, Nup159 seems to be required for ribosomal export, but to a lesser extent than for mRNA transport. Even though a Dbp5-independent function of Nup159 (Gleizes et al., 2001) cannot be excluded from these experiments, it is also well possible that Nup159 is necessary in ribosomal transport to localize Dbp5 to the cytoplasmic fibrils of the NPCs. 


\subsubsection{Dbp5 does not displace Mex67 from exported pre-ribosomal subunits}

As Dbp5 is required at the cytoplasmic side of the NPC to support the nuclear export of pre-ribosomal subunits, the question is how it contributes to this process. During mRNA export, Dbp5 is localized to the cytoplasmic fibrils and displaces bound export factors from the emerging mRNPs to provide directionality of the transport process (Lund and Guthrie, 2005; Tran et al., 2007). Thus, Dbp5 might also remodel the exported ribosomal particles and dissociate bound transport factors upon arrival in the cytoplasm. An obvious candidate to validate this hypothesis is Mex67, as its Dbp5-mediated displacement from exported mRNAs was already shown (Lund and Guthrie, 2005). Furthermore, Mex67 is also required for the transport of both preribosomal subunits (Faza et al., 2012; Yao et al., 2007), but its cytoplasmic recycling mechanism is currently unknown and could be performed by Dbp5 (Gerhardy et al., 2014). To assess this question, sucrose-density gradient centrifugation was performed to analyze the ribosomal association of Mex67 in the dbp5 mutant rat8-2. 


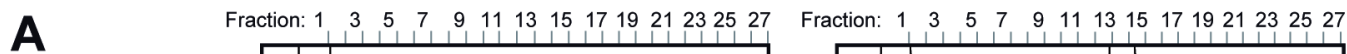
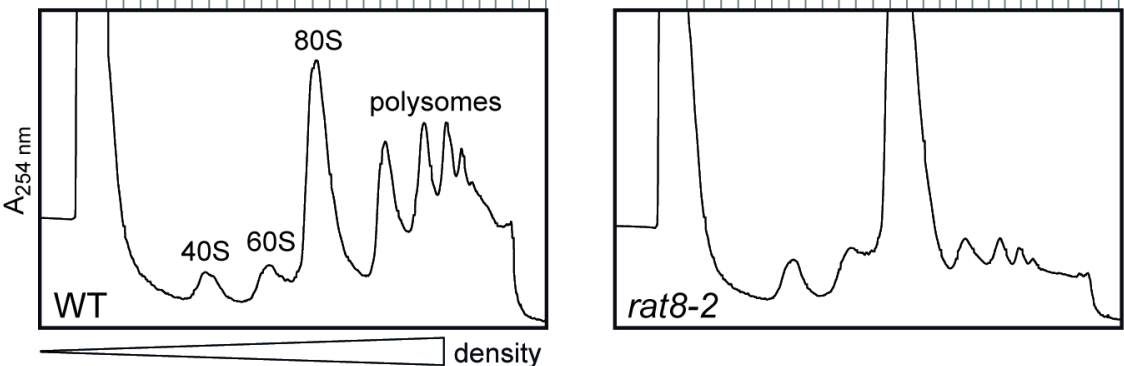

- RNase A

density
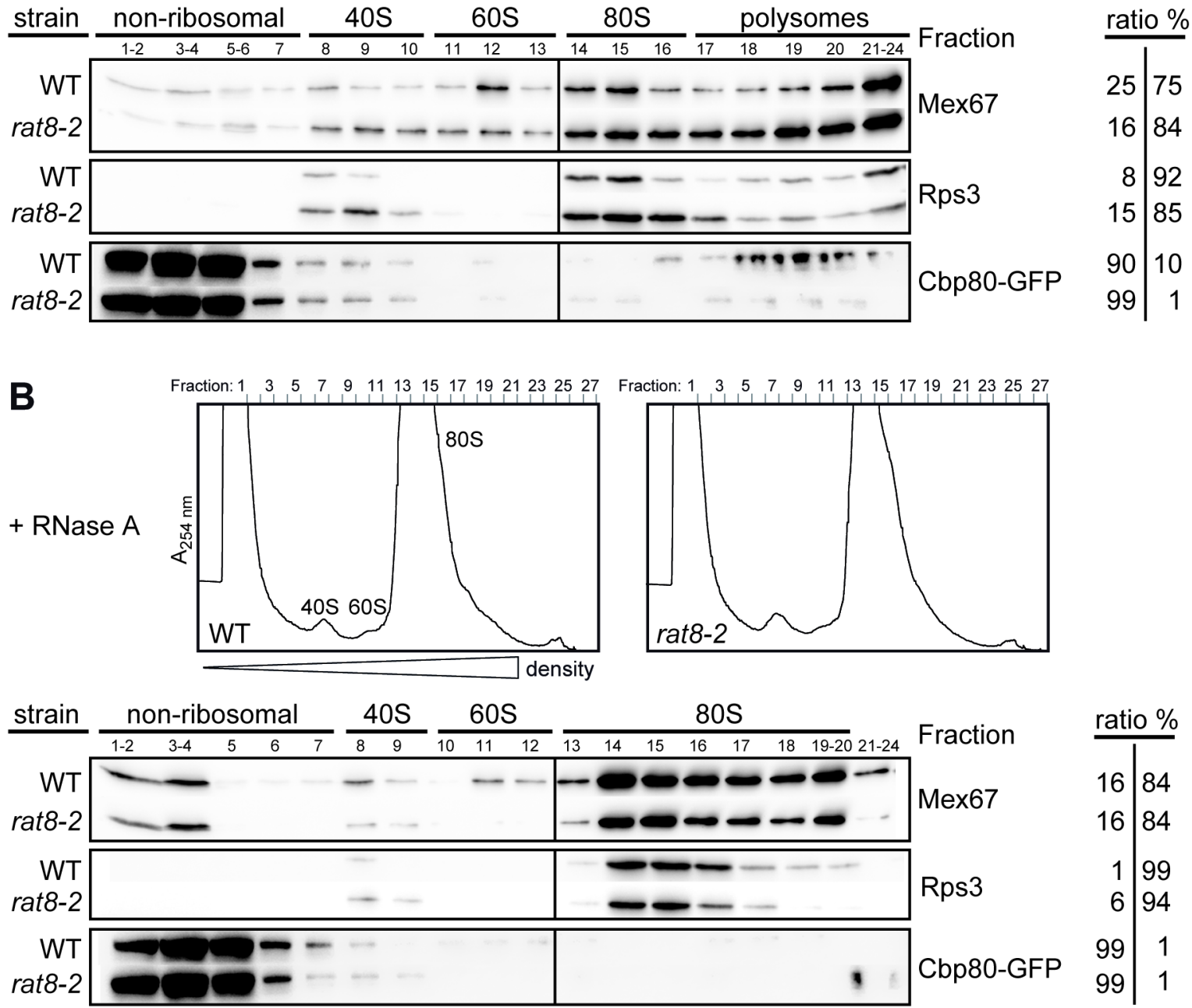

Figure 27: Mex67 is associated with polysomes and not displaced from ribosomal particles by Dbp5.

(A+B) Sucrose-density gradient fractionation experiments are shown with wild type (WT) and rat8-2 cells upon $1 \mathrm{~h}$ shift to $37^{\circ} \mathrm{C}$. After cell lysis, half of the lysate was treated with $0.25 \mathrm{mg} / \mathrm{ml}$ RNase A and the untreated (A) and treated (B) lysates were loaded onto $7-47 \%$ sucrose gradients and ultra-centrifuged. The gradients were fractionated while measuring the absorbance at $254 \mathrm{~nm}$ $\left(A_{254 n m}\right)$ resulting in the profiles shown in the upper panels. The lower panels reveal the Western blot analysis of the corresponding proteins upon TCA precipitation. Mex67 and Rps3 were detected with specific antibodies. CBP80-GFP under control of a GAL-promoter was expressed by galactose induction and the tagged protein was detected with an anti-GFP antibody. The ratio of the proteins in the light (non-ribosomal+40S+60S) and the heavy (80S+polysomal) fractions compared to the total protein levels are indicated.

In principal, the translation rate was reduced in the dbp5 mutant rat8-2 (Figure 27A). The reduction is characterized by a flattened polysome profile and an increased $80 \mathrm{~S}$ peak along with the shift of the ribosomal protein Rps3 from the polysomes- to the 
$80 \mathrm{~S}$ ribosomes-containing fractions (Figure 27A). Most likely, this reduction is caused by the mRNA export defects of rat8-2 and the decreased amount of translated mRNAs in the cytoplasm. This effect is also reflected by the shift of the cap-binding protein Cbp80, which is bound to the mRNA during the first rounds of translation (Garre et al., 2012), from the polysomal to the lighter fractions (Figure 27A).

Mex67 was part of the free 405 and 60S subunits-containing fractions of wild type and rat8-2 cells as expected for an export factor (Figure 27A). Furthermore, Mex67 was visible in the $80 \mathrm{~S}$ ribosome-associated fractions of both strains. However, the $80 \mathrm{~S}$ peak can also contain $90 \mathrm{~S}$ particles so that the association with mature $80 \mathrm{~S}$ ribosomes or 905 precursors is indistinguishable. Unexpectedly, Mex67 was also detectable in the polysomal fractions of wild type cells and its amount was even increased in rat8-2 (Figure 27A) indicating that Mex67 is either bound to the translated mRNAs or to the translating ribosomes. To distinguish between both possibilities, the lysates were treated with RNase A prior to loading of the gradients. RNase A degrades the single-stranded mRNAs and thus, causes the disruption of the polysomes and formation of all translating ribosomes in one single 805 peak (see profiles of Figure 27B). Upon RNase A treatment, the mRNA-bound Cbp80 completely shifted to the non-ribosomal fractions confirming the successful mRNA degradation (Figure 27B). Nevertheless, Mex67 was still associated with the $80 \mathrm{~S}$ ribosome-containing fractions in wild type and in rat8-2 cells (Figure 27B) showing that Mex67 is bound to the translating ribosomes even in wild type cells. Moreover, the amount of ribosome-associated Mex67 was not altered in rat8-2 compared to wild type (Figure 27B) and the same tendency was seen in initial experiments with rat8-7 (data not shown). Firstly, these results show that the enhanced amount of Mex67 in the polysomes of untreated rat8-2 cells results from the increased binding of Mex67 molecules to the translated mRNAs (Figure 27A). This increased association is most probably caused by the missing Mex67 release from the exported mRNAs in the dbp5 mutant (Lund and Guthrie, 2005). In addition, these data suggest that the ribosomal binding of Mex67, in contrast to its mRNA association is not influenced by Dbp5 and thus, Mex67 cannot be displaced by Dbp5 from the pre-ribosomal subunits.

To further support this idea, the binding of Mex67 to ribosomal proteins was analyzed in $d b p 5$ mutants by co-immunoprecipitation experiments. 

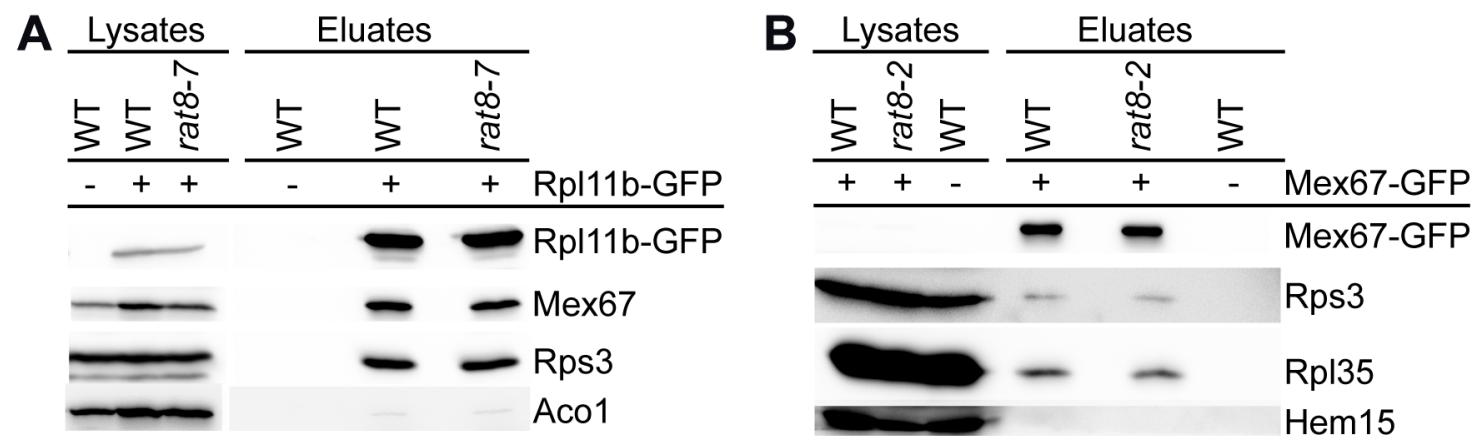

Figure 28: The ribosomal association of Mex67 is unchanged in the dbp5 mutants.

(A) Western blot analysis of an Rpl11b-GFP immunoprecipitation in wild type (WT) and rat8-7 cells reveals the same amount of co-precipitated Mex67 and Rps3 upon $1 \mathrm{~h}$ shift to $16^{\circ} \mathrm{C}$. (B) Western blot analysis shows the same levels of co-precipitated ribosomal proteins Rps 3 and Rpl35 in the Mex67-GFP pull-downs of wild type and rat8-2 cells. The cells were shifted for $1 \mathrm{~h}$ to $37^{\circ} \mathrm{C}$. (A+B) All samples were treated with RNase A. Wild type cells without tagged proteins and detection of Aco1 (Aconitase) and Hem15 (Ferrochelatase), respectively served as non-binding controls.

As seen in Figure 28A, Mex67 was equally detectable in the immunoprecipitations of the large ribosomal protein Rpl11b-GFP in wild type and rat8-7 cells. Vice versa, Mex67-GFP co-precipitated the same amounts of the small ribosomal protein Rps3 and the large ribosomal protein Rpl35 in wild type and rat8-2 cells (Figure 28B). Indeed, these data confirm that the association of Mex67 with ribosomal particles is not altered in the mutants of $D B P 5$.

In summary, Dbp5 seems to displace Mex67 only from exported mRNAs, but not from ribosomal particles revealing differences in the transport mechanism of both large ribonucleoprotein particles.

\subsubsection{The ATPase cycle of Dbp5 is dispensable for ribosomal transport}

During mRNA export, the regulated ATPase cycle of Dbp5 is necessary for the remodeling of the mRNPs at the cytoplasmic side of the NPC. The ATPase activity is stimulated by the co-factors Gle1 and IP 6 (Alcazar-Roman et al., 2006; Weirich et al., 2006) and subsequent ADP-release is enhanced by Nup159/Rat7 (Noble et al., 2011). As Mex67 is not displaced by Dbp5 from the pre-ribosomal particles, the necessity of the remodeling activity of Dbp5 and its ATPase cycle at all needs to be analyzed for ribosomal transport. To do so, the temperature-sensitive gle1-4 strain was examined for ribosomal export defects by GFP-microscopy experiments. 
A

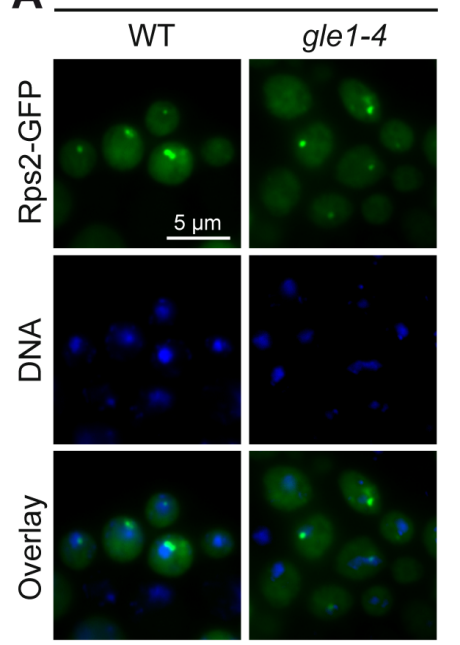

B

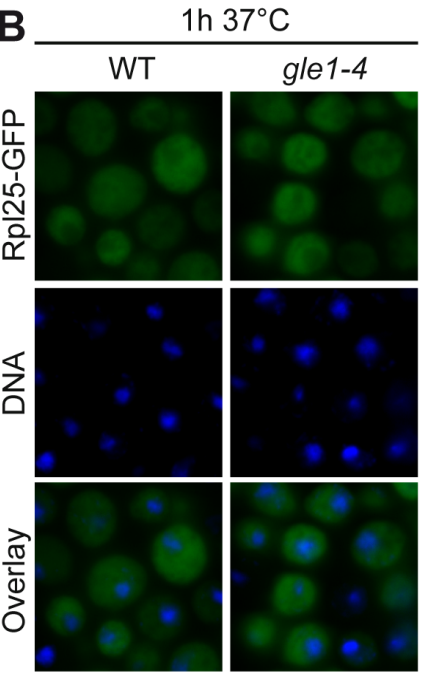

C

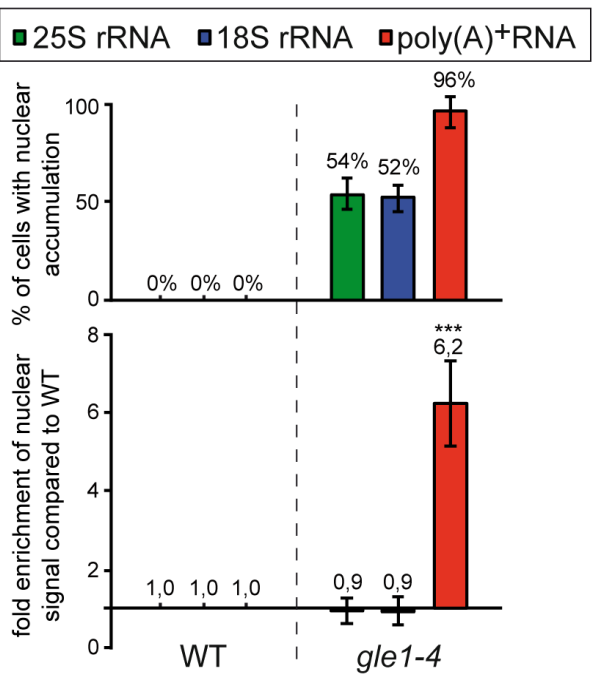

Figure 29: Missing ATPase stimulation of Dbp5 does not cause severe ribosomal export defects.

(A+B) Fluorescence microscopy pictures reveal no obvious accumulation of Rps2-GFP (A) and Rpl25-GFP (B) in the nuclei of gle1-4 cells upon $1 \mathrm{~h}$ shift to $37^{\circ} \mathrm{C}$. The DNA was stained with Hoechst. (C) Statistical analysis of FISH experiments are shown that were performed from Haijia Wu and Alexandra Hackmann (laboratory of Prof. Heike Krebber) with gle1-4 cells, as described in Figure 19. WT = wild type

The ribosomal reporter Rps2-GFP and Rpl25-GFP did not obviously accumulate in the nucleoplasm of gle1-4 cells at $37^{\circ} \mathrm{C}$ (Figure 29A+B). Single dots of Rps2-GFP were visible beside the nuclei in both, wild type and gle1-4 cells indicating that this is not a specific effect of the mutation. In addition, FISH experiments, which were performed by Haijia Wu and Alexandra Hackmann (laboratory of Prof. Dr. Heike Krebber), revealed only very slight nuclear accumulations of ribosomal RNAs (Figure 29C). Half of the analyzed cells showed a faint signal in the nucleus that was not measurable as enrichment in the quantification (Figure 29C). In contrast, the mRNA export defect was rather strong in the gle1-4 cells (Figure 29C). These results indicate that the ATPase stimulation of Dbp5 by Gle1 is only necessary for efficient mRNA transport, but not required for the nuclear export of pre-ribosomal subunits.

To further support this finding, ATPase-deficient dbp5 mutants were analyzed regarding ribosomal transport defects. In general, mutations that impair the ATPase activity of Dbp5 are lethal in yeast. However, overexpression of the ATPase-deficient $d b p 5-R 369 G$ and $d b p 5-R 426 Q$ alleles in wild type cells leads to dominant-negative effects on growth and the nuclear accumulation of mRNAs (Hodge et al., 2011). Therefore, wild type cells were transformed with plasmids containing these alleles or wild type DBP5 under control of a GAL1-promoter. The expression was induced by galactose addition for 1.5 hours and subsequently, FISH experiments were performed to examine the effect on ribosomal export. 


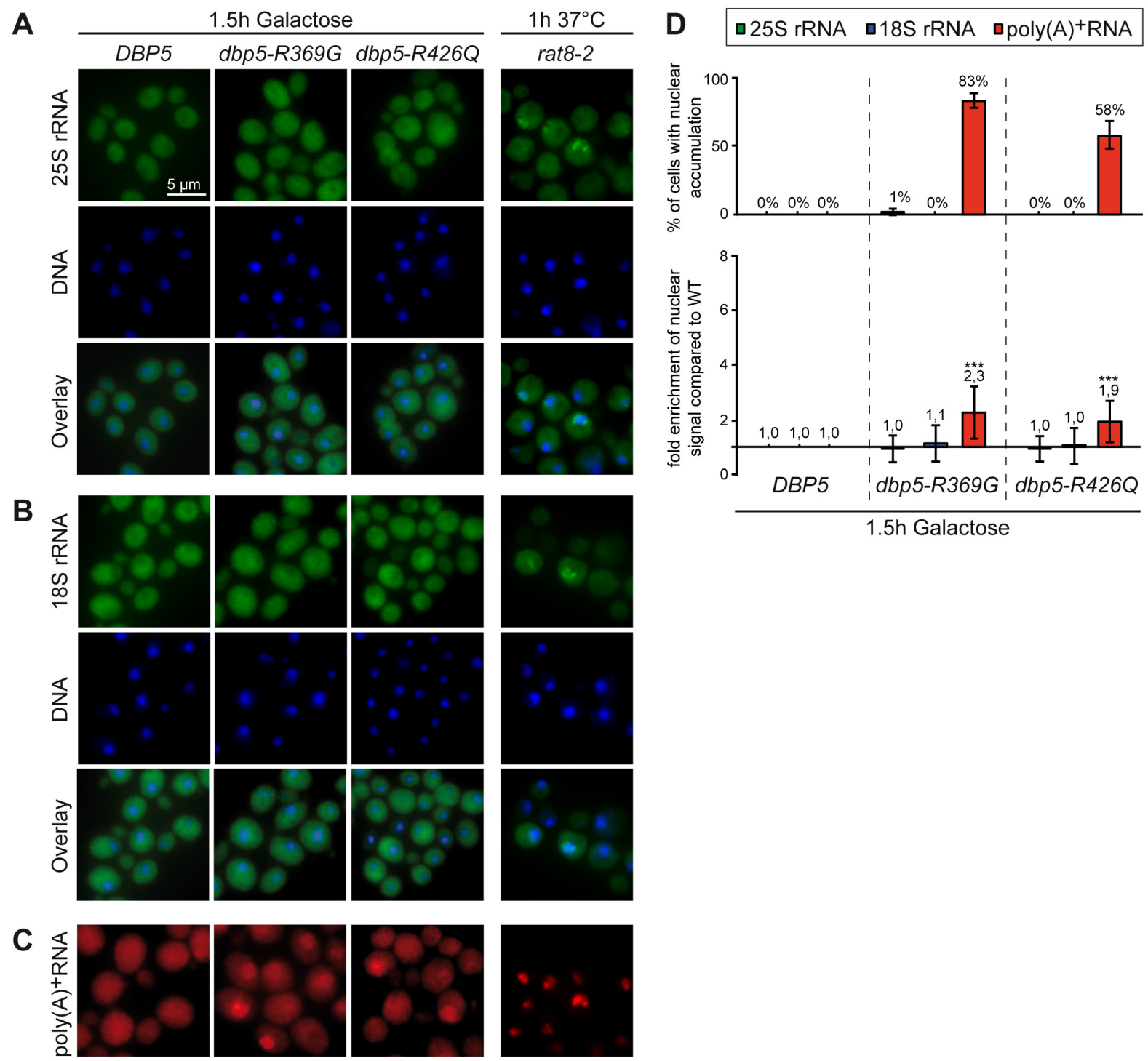

Figure 30: ATPase-deficient dbp5 mutants show no obvious ribosomal export defects.

(A-C) Fluorescence in situ hybridization experiments with probes against the 25S rRNA (A) and 18S rRNA (B) show no nuclear accumulation in wild type cells with overexpression of galactoseinducible DBP5, dbp5-R369G or dbp5-R426Q upon induction for $1.5 \mathrm{~h}$. As a positive control rat8-2 cells are shown upon $1 \mathrm{~h}$ shift to $37^{\circ} \mathrm{C}$. In contrast, poly $(A)^{+} \mathrm{RNAs}$ detected by an Cy3-labelled oligo(dT)-probe (C) accumulate in the nucleus of all mutants. The DNA was stained with Hoechst. (D) Statistical analysis of (A-C). The percentage of cells that show a nuclear accumulation of the fluorescent signal is indicated in the upper panel. The average fold enrichment of the nuclear signal of at least 10 cells compared to the whole cell and the wild type were shown. Error bars represent the standard deviation and $p$-values were calculated by an unpaired Student's t-test (type 2). $* * *=p<0.001$

Overexpression of the ATPase-deficient mutants $d b p 5-R 369 G$ and $d b p 5-R 426 Q$ for 1.5 hours did not alter the distribution of the $25 S$ and $18 S$ rRNAs compared to wild typic $D B P 5$ expressing cells (Figure $30 \mathrm{~A}+\mathrm{B}+\mathrm{D}$ ). Thus, no obvious defects in the transport of both pre-ribosomal subunits are visible. Contrary, poly(A)-tail containing RNAs accumulated in the nucleoplasm (Figure 30C+D) demonstrating that the expression of $d b p 5-R 369 G$ and $d b p 5-R 426 Q$ was indeed efficiently induced. Furthermore, the nuclear accumulation of $25 \mathrm{~S}$ and $18 \mathrm{~S}$ rRNAs in rat8-2 cells 
indicates that ribosomal export defects were successfully visualized in this assay (Figure 30A+B). These results suggest that the ATPase activity of Dbp5 in general is dispensable for ribosomal transport.

\subsubsection{Recombinant Dbp5 is able to directly bind Mex67}

The experiments performed so far suggested that Dbp5 does not displace bound export factors from the pre-ribosomal subunits in an ATPase-dependent manner to facilitate a directional ribosomal transport, as it was shown for mRNA export. Thus, how could Dbp5 support the nuclear export of ribosomal particles? One possibility might be that Dbp5 directly binds to ribosomal export factors such as Mex67 at the NPC exit and prevents by their shielding the back-sliding of the particle into the hydrophobic channel. A prerequisite for such a model would be the direct contact between Dbp5 and a ribosomal export factor. A possible candidate is Mex67, as it facilitates the transport of the large and the small pre-ribosomal subunits (Faza et al., 2012; Yao et al., 2007). Thus, the interaction of Dbp5 and Mex67 was analyzed in vivo by co-immunoprecipitation experiments. To test for a direct interaction, in vitro binding studies between recombinant Dbp5 and Mex67 were performed. As Mex67 expressed alone in E. coli was not soluble, $\mathrm{His}_{6}-\mathrm{Mtr} 2$ and Mex67 were co-expressed from one plasmid and purified as a heterodimer by a Ni-NTA pull-down, which was performed by Lysann Henker and Michael Mitter (laboratory of Prof. Krebber). GSTDbp5 and GST alone were expressed in E. coli Rosetta 2 cells using the same conditions that were already successfully tested by Irene Hampe (master thesis, laboratory of Prof. Krebber) (see section 5.12.3). The GST-Dbp5 and GST containing lysates were pre-incubated with Glutathione Sepharose beads for one hour. Subsequently, the purified heterodimeric His-Mtr2-Mex67 was added. The protein interactions were analyzed by SDS-PAGE and subsequent Coomassie staining or Western blot analyses. 
A

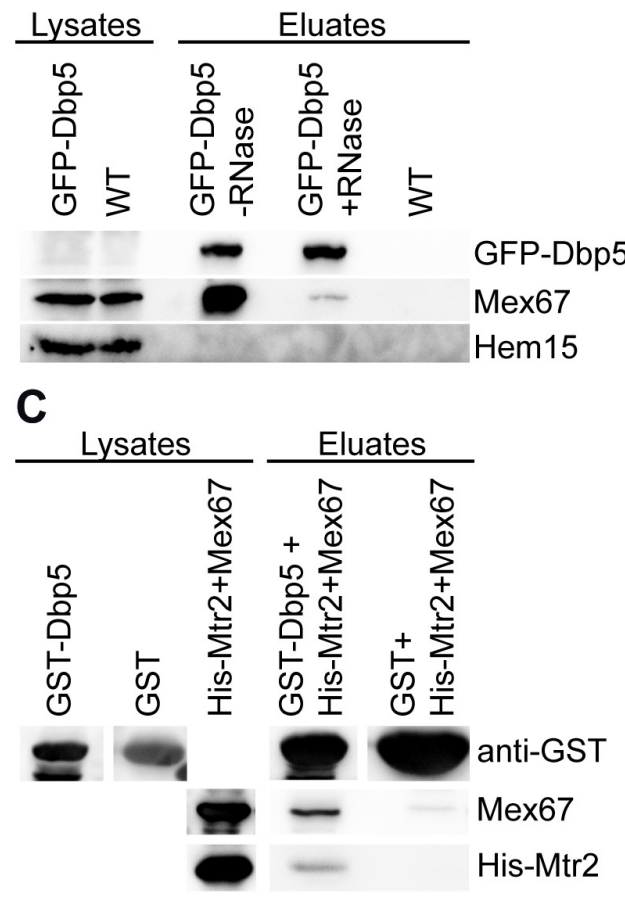

B

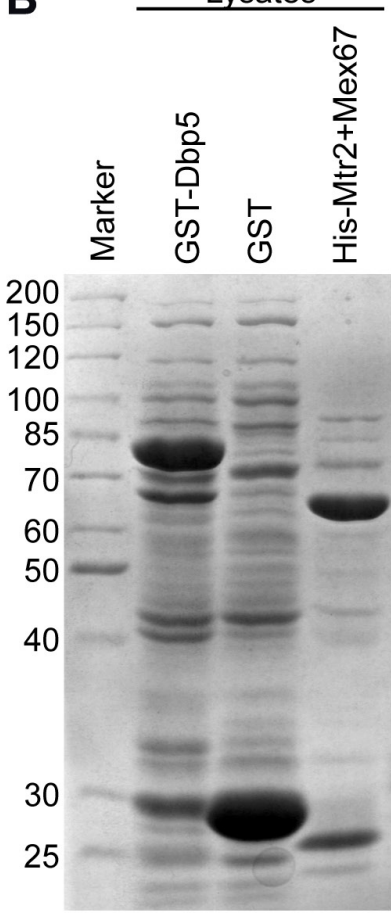

Eluates

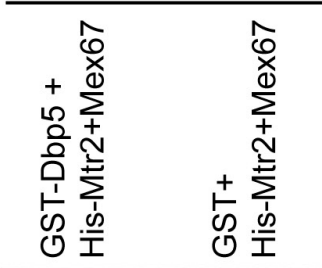

Figure 31: Dbp5 interacts with Mex67 in vivo and in vitro.

(A) The Western blot analysis of GFP-Dbp5 immunoprecipitations reveals without RNase A treatment the co-precipitation of Mex67, which is strongly decreased upon RNase A addition. Wild type (WT) cells expressing a GFP-DBP5 containing plasmid were used. Detection of Hem15 (Ferrochelatase) served as non-binding control. $(\mathbf{B}+\mathbf{C})$ In vitro binding studies show an interaction between recombinant GST-Dbp5 and heterodimeric His-Mtr2-Mex67. Glutathione Sepharose beads were pre-incubated with GST-Dbp5 or as negative control with GST containing E. coli lysates. After one hour, $30 \mu \mathrm{g}$ of the purified heterodimer His-Mtr2-Mex67 was added. The interactions were analyzed by SDS-PAGE and (B) Coomassie-staining or (C) Western blot analysis. (B) The Coomassie-stained SDS-polyacrylamid gel shows successful expression and pull-down of GST-Dbp5 and GST. (C) The Western blot analysis reveals in the GST-Dbp5 pulldown interacting bands of Mex67 and His-Mtr2. The detection was performed with an antiMex67, which recognizes also His-Mtr2, and anti-GST antibody.

Without RNase A addition to the co-immunoprecipitation, Dbp5 interacted with Mex67 in vivo, but this interaction was strongly decreased upon RNase A treatment (Figure 31A). These results show that the in vivo binding between Dbp5 and Mex67 is mainly mediated via single-stranded RNAs, most probably by their concomitant association to one mRNA molecule during mRNA export. However, a slight RNAindependent interaction between Dbp5 and Mex67 is still visible and might occur during ribosomal transport. However, whether both proteins directly bind each other or whether the interaction is mediated by other proteins or the ribosomal particles is unclear from these experiments. Therefore, in vitro binding studies were performed. In the Coomassie-stained gel, the pull-down of GST-Dbp5 and GST was visible, but also several bands below GST-Dbp5 (Figure 31B), which most likely result from degradation products. Strong bands belonging to Mex67 and His-Mtr2 were not seen in the GST-Dbp5 pull-down and potential slight interacting bands were not 
distinguishable from the degradation products (Figure 31B). Therefore, Western blot analyses were performed that detected indeed Mex67 and His-Mtr2 in the GST-Dbp5 pull-down and their bands were clearly enhanced compared to the negative control with GST alone (Figure 31C). In conclusion, these results show that Dbp5 is able to directly bind the heterodimer Mex67-Mtr2 in vitro. As the interaction was not clearly visible in the Coomassie-stained gel (Figure 31B), the proteins should not bind each other in equimolar ratios and a strong complex formation between them is unlikely. However, these data confirm that it is possible that Dbp5 shortly contacts Mex67 during ribosomal transport and might in this way support the export process.

\subsection{Analysis of Dbp5 and Rli1 in translation termination}

\subsubsection{Rli1 interacts with Dbp5 and its co-factor Gle1 during translation termination}

In the last years, the DEAD-box RNA-helicase Dbp5 (Gross et al., 2007), its ATPaseactivity stimulating co-factors $\mathrm{IP}_{6}$ and Gle1 (Alcazar-Roman et al., 2010; Bolger et al., 2008) as well as the iron-sulfur containing ABC-family ATPase Rli1 (Khoshnevis et al., 2010) were characterized as additional translation termination factors beside the eukaryotic release factors eRF1 and eRF3. All of these proteins genetically and physically interact with the canonical release factors and corresponding mutant strains show an increased stop codon read-through rate. However, until now is unclear, when Rli1, Dbp5 and Gle1 are recruited to the termination complex and whether these factors interact with each other during translation termination.

To address this question, a series of co-immunoprecipitation studies with Rli1, Dbp5 and Gle1 was performed. As a specific antibody was only available against Dbp5, yeast cells were transformed with RLI1-HA, RLI1-GFP or MYC-GLE1 containing plasmids allowing the detection of the expressed fusion proteins in the Western blot analyses. However, plasmid-borne myc-Gle1 expressed from its own promoter was not detectable. Therefore, MYC-GLE1 expression was induced by the constitutive $A D H 1$-promoter to increase the number of myc-Gle1 molecules per cell and consequently, the signals in the Western blot analyses. Importantly, overexpression and tags were not toxic for wild type cells, as confirmed by growth analysis (Figure $32 \mathrm{~A}+\mathrm{B}$ ) (Khoshnevis et al., 2010). Thus, an extra copy of RLI1 is not harmful in contrast to the significantly increased molecule number when expressed from the strong galactose promoter, which is known to reduce growth of wild type cells and to 
cause initiation defects (Dong et al., 2004).
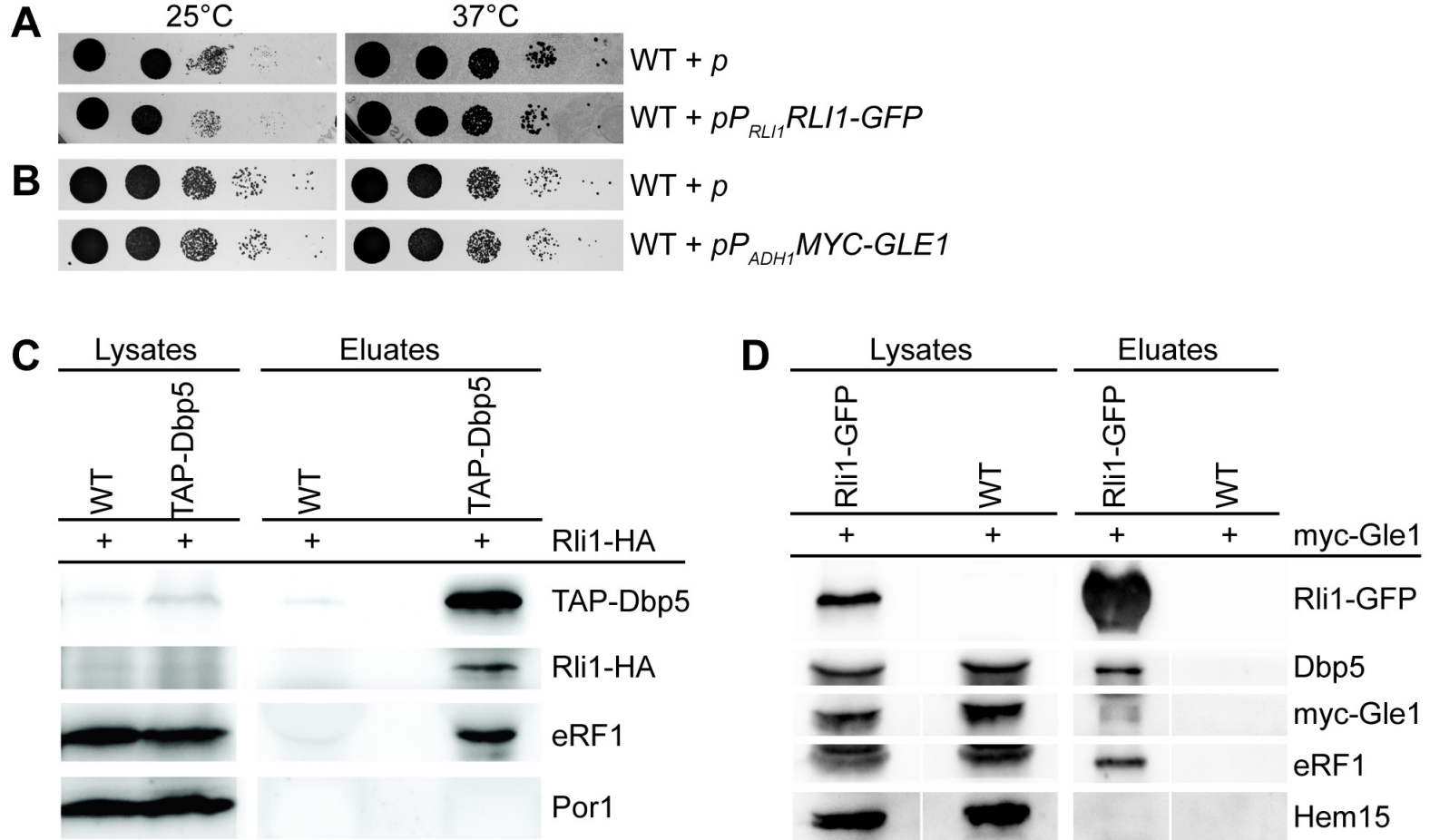

Figure 32: Rli1 interacts RNA-independently with Dbp5 and Gle1 in vivo.

(A+B) Growth analyses show no differences in the growth of wild type (WT) cells transformed either with an empty vector $(p)$ or with a plasmid containing $P_{R L I 1} R L I 1-G F P(A)$ and $P_{A D H 1} M Y C$ GLE1 (B) respectively. The cells were spotted in serial dilutions onto selective agar plates and were grown for two days at the indicated temperatures. The growth analysis in (A) was performed by Stephanie Schäfer (research assistant, laboratory of Prof. Krebber). (C) The Western blot analysis of a TAP-Dbp5 immunoprecipitation reveals co-precipitation of Rli1-HA and eRF1. A genomically tagged TAP-DBP5 strain and wild type cells transformed with a RLI1-HA containing plasmid were used. (D) The Western blot analysis shows a pull-down of plasmid borne Rli1-GFP and co-precipitation of Dbp5 and myc-Gle1. Both strains were transformed with a $P_{A D H 1} M Y C-G L E 1$ expressing plasmid. (C+D) All samples were treated with $0.2 \mathrm{mg} / \mathrm{ml}$ RNase A. Detection with specific antibodies against eRF1 served as a positive control and Por1 (Porin) and Hem15 (Ferrochelatase), respectively as non-binding controls.

Rli1-HA was co-precipitated in the TAP-Dbp5 immunoprecipitation (Figure 32C) and vice versa, endogenous Dbp5 was detectable in the Rli1-GFP pull-down (Figure 32D) revealing that both proteins are able to interact with each other in vivo. Additional immunoprecipitations with wild type cells confirmed that the co-precipitated proteins do not unspecifically bind to the IgG-sepharose or to the GFP-Trap beads, respectively (Figure 32C+D). Moreover, the specificity of the co-immunoprecipitation experiments was also verified by the detection of the unrelated mitochondrial proteins Por1 and Hem15, which did not associate with TAP-Dbp5 or Rli1-GFP, respectively (Figure 32C+D). The successful detection of co-precipitated eRF1 in both immunoprecipitations (Figure 32C+D) confirms the already published interactions of Dbp5 and Rli1 with this canonical release factor (Gross et al., 2007; Khoshnevis et al., 2010). 
As all samples were treated with RNase A to degrade single-stranded RNAs, the contact between Rli1 and Dbp5 should not be mediated by mRNAs, but rather by protein complexes or ribosomal particles. However, whether Rli1 directly binds to Dbp5 or both proteins interact via their simultaneous binding to the ribosome cannot be judged, because these studies were performed with complete yeast cell lysates.

Furthermore, the Rli1-GFP immunoprecipitation showed a co-precipitation of Gle1 (Figure 32D) indicating that Rli1 also interacts RNA-independently with the ATPaseactivity stimulating co-factor of Dbp5. To support these data, these experiments were repeated by Simon Uhse and obtained similar results (master thesis, laboratory of Prof. Heike Krebber). In summary, these data demonstrate that the additional termination factors Dbp5, its co-factor Gle1 and Rli1 are able to form a complex in vivo that might take place during translation termination.

However, these multifunctional factors could also interact during other cellular processes such as ribosomal transport with each other. To distinguish between these possibilities, their interaction was analyzed in the temperature-sensitive eRF1 mutant sup45-2, which is defective in translation termination. Upon temperature shift to $37^{\circ} \mathrm{C}$, the mutant sup45-2 protein is not able to associate with ribosomes anymore resulting in disturbed recognition of stop codons and their increased read-through (Stansfield et al., 1997).
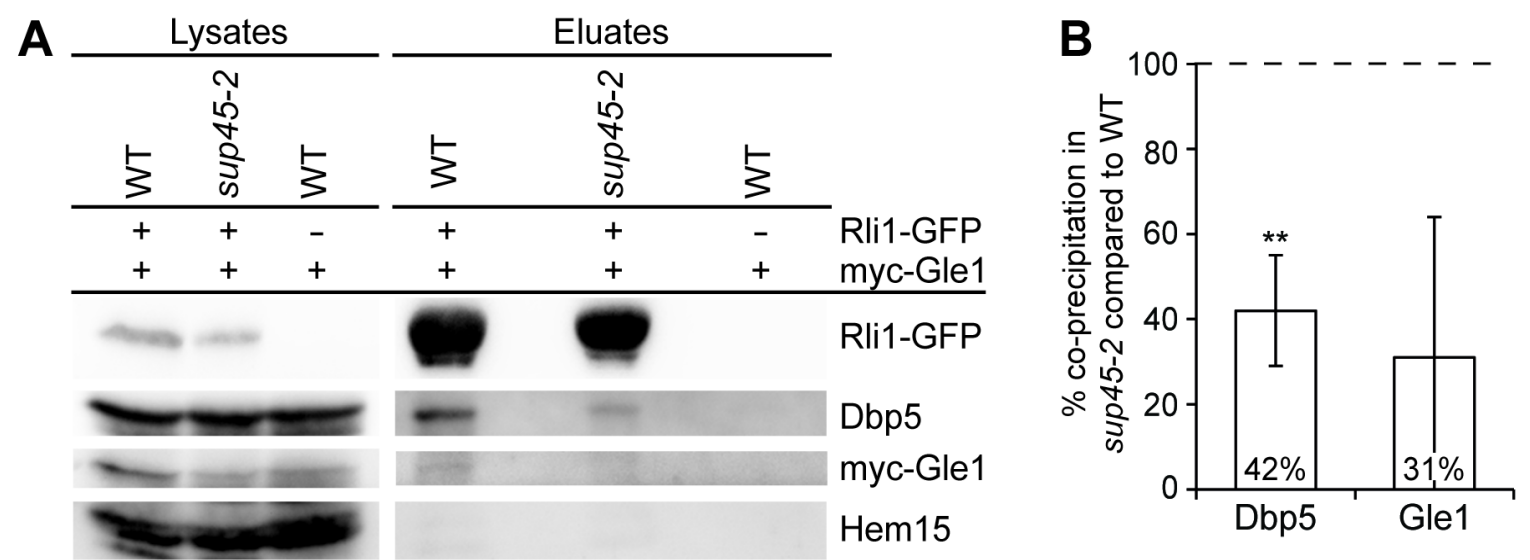

Figure 33: The interaction of Rli1 with Dbp5 and Gle1 is decreased without functional eRF1. (A) The Western blot analysis shows less Dbp5 and Gle1 co-precipitation in the Rli1-GFP pulldown of sup45-2 cells compared to wild type (WT) cells upon $1 \mathrm{~h}$ shift to $37^{\circ} \mathrm{C}$. All coimmunoprecipitations were treated with $0.2 \mathrm{mg} / \mathrm{ml}$ RNase A. Both strains were transformed with $P_{A D H 1}$ MYC-GLE1 and RLI1-GFP containing plasmids. Hem15 (Ferrochelatase) served as negative control and was detected with a direct antibody. (B) Statistic analysis of the interactions in (A). The intensities of the co-precipitated bands in sup45-2 were related to the Rli1-GFP pull-down and to the ratios in the wild type. Four experiments were quantified for the Dbp5 and two for the Gle1 coprecipitation and the resulting mean values and standard derivations are shown. The data arise from the present work and from one experiment of Simon Uhse (master thesis, laboratory of Prof. Krebber). The $p$-values were calculated with an unpaired Student's t-test (type 3 ): ${ }^{\star \star}=p<0.01$. 
In comparison to wild type cells, less Dbp5 and myc-Gle1 was co-precipitated by the Rli1-GFP pull-down in sup45-2 cells (Figure 33A). Thus, quantification of four independent experiments revealed that the in vivo interaction between Rli1 and Dbp5 was on average decreased to $\sim 42 \%$ in the sup45-2 strain compared to wild type cells (Figure 33B). A similar tendency was seen for Gle1 (Figure 33B), but only two experiments were evaluable, because the protein was hardly detectable in the Western blot analyses despite expression from the constitutive $A D H 1$-promoter (Figure 33A). In conclusion, these results show that functional eRF1 and ongoing translation termination are necessary to facilitate the entire in vivo interaction between Rli1 and Dbp5 and most likely between Rli1 and Gle1 as well. Nevertheless, a sub-fraction of these translation termination factors was still able to contact each other independently of eRF1.

To confirm that the proceeding of translation termination is indeed required for the binding of Rli1 to Dbp5-containing complexes, wild type cells transformed with RLI1-GFP expressing plasmids were treated for $25 \mathrm{~min}$ with $0.1 \mathrm{mg} / \mathrm{ml}$ cycloheximide before cell lysis and subsequent co-immunoprecipitation studies. Cycloheximide is an antibiotic that inhibits translation elongation (Masek et al., 2011) and in this way prevents the arrival of the translating ribosomes at the stop codon.

A

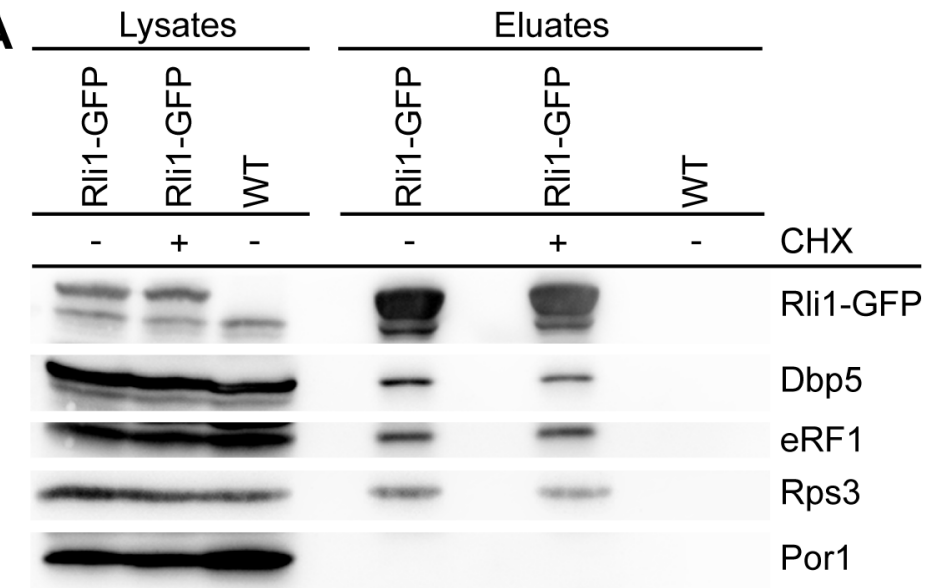

B

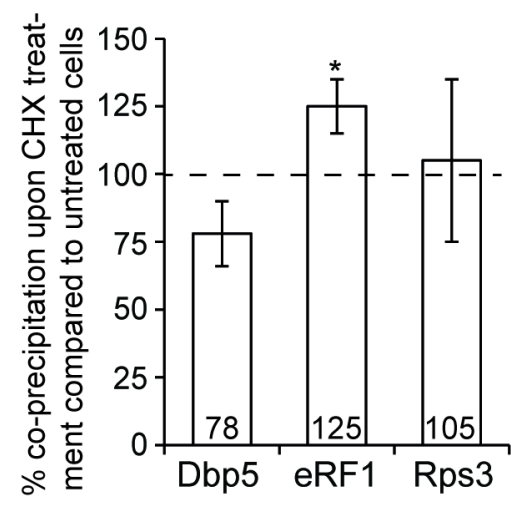

Figure 34: The interaction between Rli1 and Dbp5 is slightly decreased without proceeding of translation elongation and termination.

(A) The Western blot analysis shows a co-immunoprecipitation experiment after RNase A treatment with pull-downs of plasmid-borne Rli1-GFP. Half of the cells were treated for 25 min with $0.1 \mathrm{mg} / \mathrm{ml}$ cycloheximide (CHX) before harvesting. Co-precipitated Dbp5, eRF1, Rps3 and as a negative control Por1 (Porin) were detected with specific antibodies. (B) Statistical analysis of the interactions in (A). The amount of co-precipitated Dbp5, eRF1 and Rps3 was quantified and related to the pull-down of Rli1-GFP. The resulting ratios of treated were related to untreated cells. The average of three independent experiments and standard deviations are shown. The $p$-values were calculated with an unpaired Student's t-test (type 3): ${ }^{*}=0<0.05$ 
Upon cycloheximide treatment, the Rli1-GFP immunoprecipitation revealed a slightly decreased co-precipitation of Dbp5 (Figure 34A), which was on average reduced to $78 \%$ compared to untreated cells (Figure 34B). This tendency was visible in three independent experiments, but a further repetition might be necessary for a statistically significant result (Figure 34B). However, these first data show that the interaction between Rli1 and Dbp5 is indeed affected by the cycloheximide-mediated inhibition of translation elongation and termination. Additionally, the detection of coprecipitated eRF1 and Rps3 in the Rli1-GFP immunoprecipitation reveals that the cycloheximide treatment did not generally decrease the amount of Rli1-GFP associated proteins (Figure 34A). In fact, the quantification shows that the binding of Rli1 to the ribosomal protein Rps3 was not significantly altered by the cycloheximide addition (Figure 34B). In contrast, the interaction between Rli1 and eRF1 was marginally, but significantly enhanced to a mean of $125 \%$ compared to untreated cells (Figure 34B). Thus, the slightly reduced association of Dbp5 with Rli1containing particles should be a specific effect of the cycloheximide treatment. A further decrease of this interaction was visible after raising the cycloheximide concentration to $0.5 \mathrm{mg} / \mathrm{ml}$ in an initial experiment (data not shown), which should be repeated. However, this preliminary result indicates that increased amounts of cycloheximide might be necessary to completely prevent translation elongation and termination and thus, the complex formation between Dbp5 and Rli1. Nevertheless, a sub-fraction of both proteins might also interact during another cellular process.

In summary, the data from Figure 33 and Figure 34 suggest that an interaction of Dbp5 and Rli1 during eRF1-mediated translation termination is indeed possible, but might not exclusively take place during this process.

\subsubsection{The ribosomal association of Dbp5 is dependent on functional eRF1}

However, until now is unclear, how Dbp5 is delivered to the translation termination process. On the one hand, Gross et al. (2007) suggested that Dbp5 might enter together with eRF1 the ribosomal A-site that contains a termination codon. On the other hand, Dbp5 could be already bound to the translating ribosomes and awaits the arrival of eRF1 at the stop codon. To distinguish between these possibilities, the association of Dbp5 with ribosomal proteins of the $60 \mathrm{~S}$ and the $40 \mathrm{~S}$ subunit was analyzed in the temperature-sensitive eRF1 mutant sup45-2 and compared to wild type cells. For that, co-immunoprecipitation experiments were performed with both strains that were transformed with GFP-DBP5 expressing plasmids. 

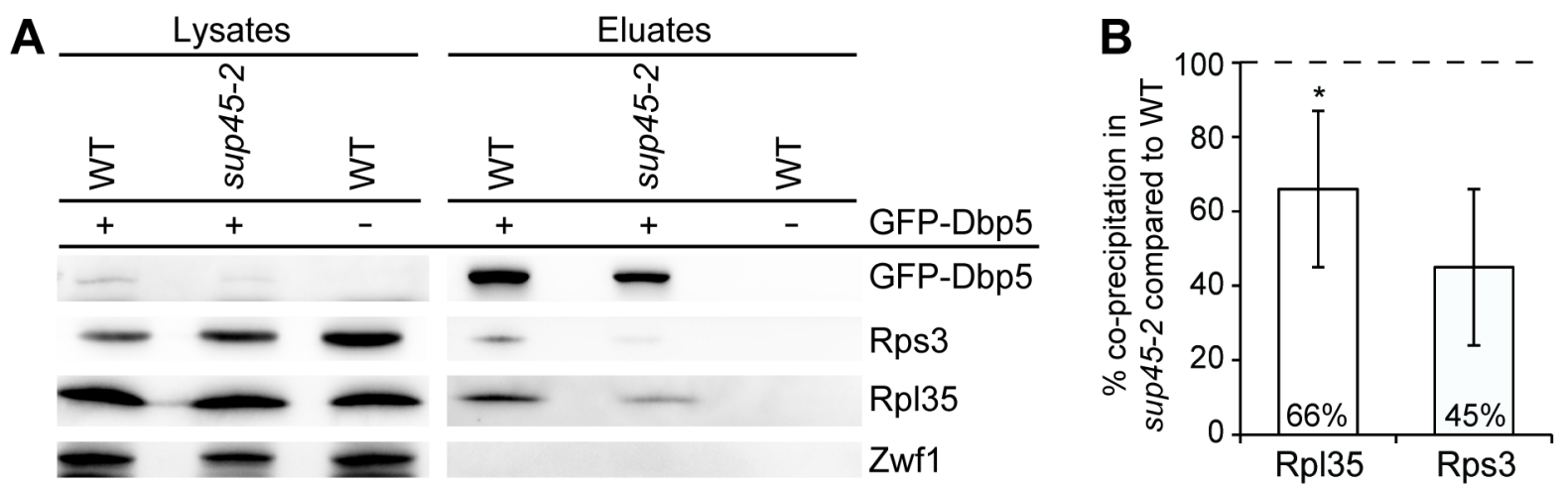

Figure 35: The binding of Dbp5 to ribosomal proteins is decreased without functional eRF1.

(A) The Western blot analysis of GFP-Dbp5 immunoprecipitations shows less co-precipitation of the small ribosomal protein Rps3 and the large ribosomal protein Rpl35 in sup45-2 compared to wild type (WT) cells. All cells were shifted for $1 \mathrm{~h}$ to $37^{\circ} \mathrm{C}$ and all immunoprecipitations were treated with $0.2 \mathrm{mg} / \mathrm{ml}$ RNase A. The ribosomal proteins and Zwf1 (Glucose-6-phosphate dehydrogenase) as negative control were detected with specific antibodies. (B) Statistic analysis of the interactions in (A). Quantification of the co-precipitated bands was performed and related to the GFP-Dbp5 pulldown and the wild type. The average of four experiments for Rpl35 and of two experiments for Rps3 is shown with the corresponding standard deviation and $p$-values calculated by an unpaired Student's t-test (type 3 ). ${ }^{*}=p<0.05$

As expected for a translation termination factor, the ribosomal proteins Rps3 from the $40 S$ subunit and Rpl35 from the 60S subunit are detectable in the GFP-Dbp5 immunoprecipitation of wild type cells (Figure 35A). These interactions suggest a binding of Dbp5 to the ribosomal subunits or $80 \mathrm{~S}$ ribosomes rather than to translated mRNAs, because all samples were treated with RNase A for mRNA degradation. Interestingly, compared to wild type cells, less Rps3 and Rpl35 was co-precipitated with GFP-Dbp5 in sup45-2 cells upon temperature shift (Figure 35A) indicating that the ribosomal association of Dbp5 is reduced without functional eRF1. The quantification of four independent experiments reveals that the binding between Rpl35 and Dbp5 is statistically significantly decreased to a mean of $66 \%$ in the sup45-2 strain (Figure 35B). A similar reduction to a mean of $45 \%$ is seen for the interaction between Rps3 and Dbp5, but only two experiments were evaluable due to the difficult detection of Rps3 in the Western blot analyses (Figure 35B). In summary, the reduced association of Dbp5 with Rpl35- and Rps3-containing particles, which might represent the $80 \mathrm{~S}$ ribosomes, in sup45-2 cells indicates that eRF1 could indeed be required for the recruitment of Dbp5 to the terminating ribosomes.

Nevertheless, as complete yeast lysates were used for these experiments, the ribosomal particles that are bound by Dbp5 cannot be exactly estimated. For a profound analysis of the association of Dbp5 with the different ribosomal particles in sup45-2 cells, sucrose-density gradient fractionation experiments were conducted. These were initial experiments that should be repeated to obtain solid results. 

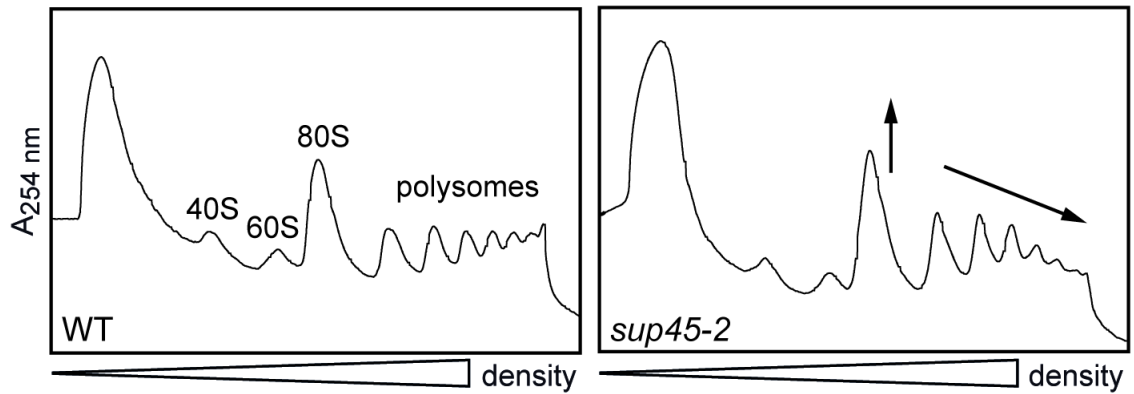

Figure 36: The eRF1 mutant sup45-2 shows a polysome run-off and an increased 80S peak. Polysome profiles of wild type (WT) and sup45-2 cells are shown upon $30 \mathrm{~min}$ shift to $37^{\circ} \mathrm{C}$. $10 \mathrm{OD}_{260 \mathrm{~nm}}$ units of the lysates were loaded onto linear 6-46\% sucrose-gradients und ultracentrifuged. The profiles are measured by flow through photometry at an absorbance of $254 \mathrm{~nm}$ $\left(A_{254 n m}\right)$ while fractionation of the gradients. Arrows mark the changes in the profile of sup45-2 compared to wild type.

Interestingly, the polysome profile of temperature shifted sup45-2 cells showed a polysome run-off and a concomitantly increased $80 \mathrm{~S}$ peak (Figure 36), which is generally a typical phenotype of cells defective in translation initiation (Masek et al., 2011). Thus, even though sup45-2 is primarily defective in translation termination, the rate of translation is also decreased and should be considered in the analyses of protein-interactions.

For the efficient detection of associated proteins in the different fractions, threefold more yeast cell lysate was loaded onto the sucrose-gradients leading to the profiles seen in Figure 37A. Nevertheless, the polysome run-off and the increased $80 \mathrm{~S}$ peak were visible as in Figure 36.

A
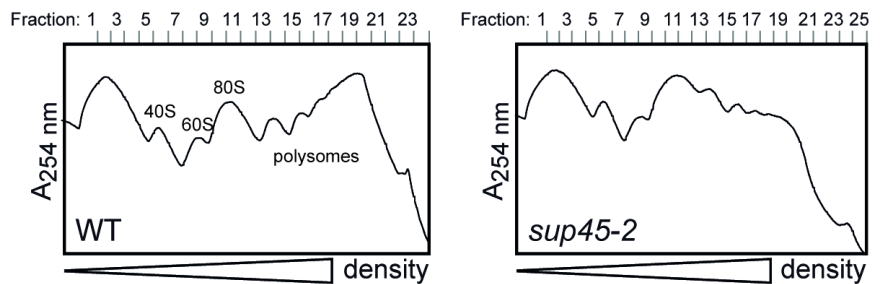

B

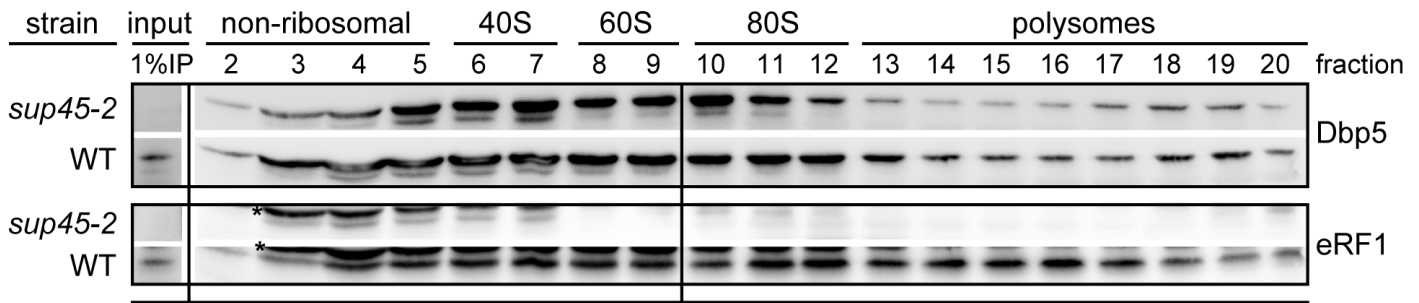

sup45-2

WT
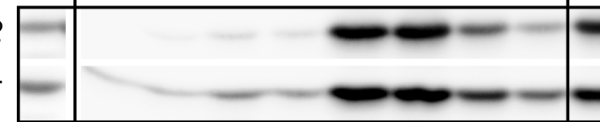

Figure 37: Dbp5 is less associated with the ribosomal fractions of sup45-2 cells.

(A) Polysome profiles of wild type (WT) and sup45-2 cells are shown upon 30 min shift to $37^{\circ} \mathrm{C} .30$ $\mathrm{OD}_{260 \mathrm{~nm}}$ units of the lysates were loaded onto linear 10-50\% sucrose gradients, ultra-centrifuged and fractionated while measuring the absorbance at $254 \mathrm{~nm}\left(A_{254 \mathrm{~nm}}\right)$ leading to the presented profiles. (B) The Western blot analysis of the corresponding protein fractions of (A) are shown after TCA-precipitation, separation of the complete fractions by SDS-PAGE and detection with direct antibodies. Ratios of Dbp5 and Rps3 in the light (non-ribosomal+40S+60S) and in the heavy (80S+polysomal) fractions compared to total protein are indicated. * = upper band belongs to Dbp5 
In wild type cells, eRF1 and Dbp5 were detectable throughout the whole gradient, as already published previously (Eyler et al., 2013; Gross et al., 2007; Stansfield et al., 1992). In contrast, the mutated sup45-2 protein was nearly completely removed from the ribosomal fractions after shifting the sup $45-2$ cells to $37^{\circ} \mathrm{C}$ for 30 min (Figure 37B) according to its known defect in ribosome binding (Stansfield et al., 1997). Consequently, Dbp5 was $\sim 10 \%$ less detectable in the single 80 s ribosomes (monosomes) and polysomes-containing fractions of sup45-2 cells compared to the wild type (Figure 37B) indicating that the ribosome binding of Dbp5 is indeed slightly reduced in the eRF1 mutant. Especially, the amount of Dbp5 that is associated with $80 \mathrm{~S}$ monosomes must be decreased considering the enlarged 805 peak in sup45-2 cells (Figure 36 and Figure 37A). This effect was even enhanced upon longer temperature shift (data not shown). In contrast, the association of Dbp5 with the free $40 \mathrm{~S}$ and $60 \mathrm{~S}$ subunits was not visibly altered in sup45-2 cells (Figure 37B) suggesting that Dbp5 is still able to contact the free ribosomal subunits independent of functional eRF1.

Thus, Dbp5 might be recruited by eRF1 to the $80 \mathrm{~S}$ ribosomes. However, the decreased ribosomal recruitment of Dbp5 in the eRF1 mutant sup45-2 might also be an effect of its generally reduced translation rate. Therefore, a ribosomal recruitment of Dbp5 during another part of the translation process cannot be excluded.

\subsubsection{The association of Rli1 with ribosomal proteins is not decreased without functional eRF1}

As for Dbp5, the time point of the Rli1 recruitment to the termination complex needs further investigations. Crystal structure analyses indicated that Rli1 and eRF3 share the binding site on the terminating ribosomes so that their binding must be mutually exclusive (Becker et al., 2012). Thus, Rli1 might take over the position of eRF3 upon its dissociation and might stabilize the optimal position of eRF1 in the peptidyltransferase center for peptidyl-tRNA hydrolysis (Becker et al., 2012; Shoemaker and Green, 2011). Thus, Rli1 could be recruited to the terminating ribosome at this time point or Rli1 might be already associated with the translating ribosome at another binding site. Therefore, co-immunoprecipitation experiments were performed to analyze the in vivo association of Rli1 with ribosomal proteins in the terminationdefective sup45-2 mutant. 

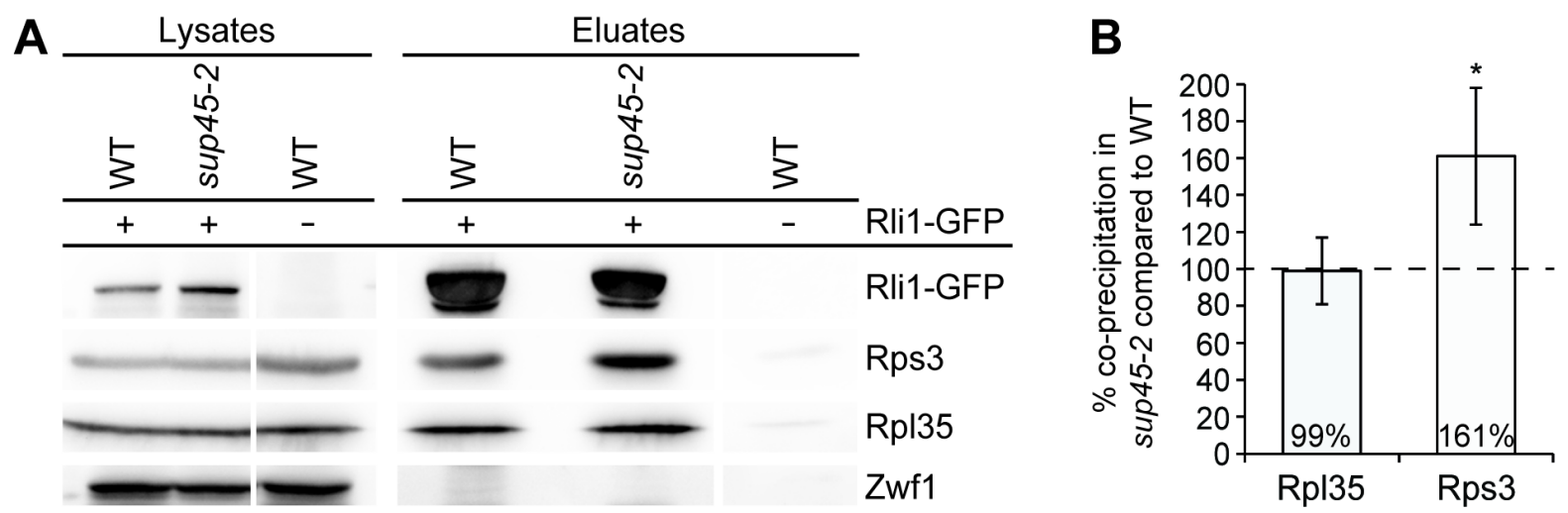

Figure 38: Rli1 binds stronger to Rps3, but its binding to Rpl35 is unchanged in sup45-2.

(A) The Western blot analysis of Rli1-GFP immunoprecipitations reveals decreased co-precipitation of the small ribosomal protein Rps3 in sup45-2 compared to wild type (WT) upon 30 min shift to $37^{\circ} \mathrm{C}$. The level of co-precipitated large ribosomal protein Rpl35 is unchanged. Detection of Zwf1 (Glucose-6-phosphate dehydrogenase) served as a non-binding control. The samples were treated with RNase A. (B) Statistical analysis of (A). Four independent experiments were quantified and the level of co-precipitation related to the pull-down and the wild type ratio. The average is shown with standard deviation and $p$-value calculated by an unpaired Student's t-test (type 3). ${ }^{*}=p<0.05$

The amount of co-precipitated Rps3 in the Rli1-GFP immunoprecipitations was increased to a mean of $161 \%$ in sup45-2 compared to wild type cells (Figure 38). This result suggests that Rli1 binds enhanced to small ribosomal subunits in the eRF1 mutant. In contrast, the co-immunoprecipitation of the large ribosomal protein Rpl35 was not significantly altered in sup45-2 cells (Figure 38) indicating that the association of Rli1 to the $60 \mathrm{~S}$ subunit is not changed contrary to the $40 \mathrm{~S}$ subunits in the eRF1 mutant. Thus, the ribosomal recruitment of Rli1 might not depend on functional eRF1 and ongoing of translation termination. 


\section{DISCUSSION}

\subsection{The DEAD-box RNA-helicase Dbp5 is ATPase-independently required for the nuclear export of both pre-ribosomal subunits}

The DEAD-box RNA-helicase Dbp5 is well-known as an mRNA export factor that is essential for the directional transport of mRNAs from the nucleus into the cytoplasm (Snay-Hodge et al., 1998; Tseng et al., 1998). By using its ATPase cycle, Dbp5 remodels emerging mRNPs at the cytoplasmic sides of the NPCs and contributes by the displacement of mRNA-bound transport factors such as Mex67 and Nab2 to their irreversible export (Lund and Guthrie, 2005; Tran et al., 2007). In addition to this established function, the present study characterizes a novel role of Dbp5 in the nuclear export of both pre-ribosomal subunits. But remarkably, the ATPasedependent remodeling activity of Dbp5 is dispensable for this process revealing differences in its transport mechanism of mRNPs and ribosomal complexes.

\subsubsection{Dbp5 is directly required for the nuclear export of pre-60S and pre-40S particles}

Different temperature-sensitive mutants of DBP5 accumulate ribosomal proteins and rRNAs from both pre-ribosomal subunits and in addition the pre-60S export factor Nmd3 in the nucleoplasm (Figure 18 and Figure 19). These results indicate that Dbp5 is required for efficient transport of both, the pre-40S and pre-60S particles from the nucleus into the cytoplasm. In support of these data, the dbp5 mutants rat8-1 and rat8-2 were already listed in a large-scale export study screening for yeast mutants that mislocalize the 60S-reporter Rpl11b-GFP in the nucleoplasm (StageZimmermann et al., 2000). However, the authors did not publish any result of their biogenesis analyses and the effect of the $d b p 5$ mutants on the pre-60S transport was not further characterized. Thus, a detailed study about how Dbp5 impacts the nuclear export of the pre-60S particles was still missing.

In contrast to the large subunit, a nuclear accumulation of small pre-ribosomal particles in dbp5 mutants has not been described before. Moy and Silver (1999) found no defects for rat8-1 in their pre-40S export assay. This result is in agreement with the present study, which reveals a slight nuclear accumulation of the 18S rRNA in only $5 \%$ of the rat8-1 cells (Figure 19). However, Gleizes et al. (2001) did not 
detect pre-40S export defects in rat8-2 cells as well, although this mutant shows a strong $18 \mathrm{~S}$ rRNA mislocalization at $37^{\circ} \mathrm{C}$ in our assay (Figure 19). The main difference between both studies is that Gleizes et al. (2001) shifted the cells only to a temperature of $34^{\circ} \mathrm{C}$ to avoid fragmentation of the nucleolus in the export mutants. However, this temperature might be too low to induce the ribosomal transport defects in rat8-2. Certainly, nucleolar fragmentation might occur in mRNA export mutants at $37^{\circ} \mathrm{C}$, but it is unlikely that this effect is the only reason for the enhanced nuclear signals of ribosomal proteins and rRNAs in our assays, because not all strains with nucleolar fragmentation show a strong ribosomal export defect, for example gle1-4 (Figure 29) (Murphy and Wente, 1996) or rat7-1 (Figure 26) (Del Priore et al., 1996). Thus, the detected nuclear accumulation of 185 rRNAs and of the 40S reporter Rps2-GFP in several dbp5 mutants (Figure 18 and Figure 19) indicates that Dbp5 is also required for efficient pre-40S export.

However, the observed nuclear mislocalization of both pre-ribosomal particles (Figure 18 and Figure 19) could also be caused by other effects than the direct involvement of Dbp5 in ribosomal transport. For example, as many of the mRNAs, which accumulate in the nucleus of $d b p 5$ mutants, code for ribosomal proteins (Perez-Ortin et al., 2011), the known mRNA export defects might also affect the ribosomal transport. If this is true, less ribosomal proteins should be expressed in the cytoplasm and imported into the nucleolus and the subsequent lack of these ribosomal proteins would lead to an accumulation of ribosomal precursor molecules in the nucleolus. In this case, only the rRNA probes, but not the GFP-tagged ribosomal proteins would generate an enhanced signal in the nucleolus. However, in our assays all molecules are affected and accumulate in the nucleoplasm of $d b p 5$ mutants (Figure 18 and Figure 19). Thus, the prolonged block of mRNAs in the nucleus might lead to a reduced amount of available ribosomal proteins, but this effect seems not to be the primary cause for the ribosomal transport defects in $d b p 5$ mutants after one hour temperature shift. Another indirect effect of the mRNA export defects, which could induce the nuclear retention of ribosomal particles, might be the lack of free Mex67, which is the common export factor for the transport of mRNAs and of pre-ribosomal subunits (Faza et al., 2012; Segref et al., 1997; Yao et al., 2007). It is known that Mex67 is not displaced from exported mRNAs in dbp5 mutants (Lund and Guthrie, 2005) and due to this mRNA tethering, not enough free Mex67 molecules might be available to facilitate ribosomal transport. If this would be the case, the intensity of the mRNA export defect would correlate with the extent of ribosomal mislocalization 
in all dbp5 mutants. However, such correlation could not be observed (Figure 19). In summary, it remains unlikely that the nuclear accumulation of pre-ribosomal particles is only caused by the mRNA export defects in the $d b p 5$ mutants. In general, as other mRNA export mutants such as rat7-1/nup159 and gle1-4 do not also show strong ribosomal mislocalizations (Figure 26 and Figure 29), defects in the nuclear export of mRNAs cannot principally induce severe ribosomal transport defects.

Another explanation for the observed nuclear accumulations might be defects in the biogenesis of ribosomal subunits (Tschochner and Hurt, 2003). Indeed, minor changes in the steady state level of different rRNA precursors are visible in the strongest ribosomal transport mutants rat8-2 and rat8-7 (Figure 20). These differences might be caused by a slightly delayed 35S rRNA processing, as an increased amount of the 35S transcript and less following intermediates are detectable. Furthermore, the appearance of an aberrant 23S rRNA (Figure 20) is also characteristic for a deceleration in the early processing steps and results from a premature $A_{3}$ cleavage of the $35 \mathrm{~S}$ transcript prior to splitting at the sites $A_{0}, A_{1}$ and $A_{2}$ (Figure 1B) (Gallagher et al., 2004; Pertschy et al., 2007; Venema and Tollervey, 1999). However, the observed variations are also visible and already described in the established ribosomal export mutants xpo1-1 and rat7-1/nup159 (Figure 20) (Del Priore et al., 1996; Gleizes et al., 2001; Moy and Silver, 1999). It remains unlikely that all of these export factors are involved in the rRNA processing in the nucleus. This is especially true for Nup159, which is exclusively located in the cytoplasm as part of the cytoplasmic fibrils of the NPC (Del Priore et al., 1996). Thus, slight alteration in rRNA processing might be a typical phenotype of ribosomal export mutants and could be an indirect consequence of their transport defects. Indeed, due to the tight connection of ribosomal biogenesis and export it is difficult to distinguish between primary biogenesis and export defects (Tschochner and Hurt, 2003). Unexpected for ribosomal transport mutants, the steady state level of the $20 \mathrm{~S}$ prerRNA is not significantly increased in xpo1-1, rat7-1 and the dbp5 mutants (Figure 20). The cleavage of the $20 \mathrm{~S}$ pre-rRNA and generation of the mature $18 \mathrm{~S}$ rRNA occurs in the cytoplasm (Udem and Warner, 1973) and thus, an accumulation of the precursor would be expected in mutants defective in the nuclear pre-40S export. However, this effect might be a consequence of the slightly delayed 35S pre-rRNA processing and the appearance of an aberrant 23S rRNA (Figure 20), as this intermediate is no substrate for the processing machinery and consequently less $20 \mathrm{~S}$ pre-rRNAs might be principally produced (Venema and Tollervey, 1999). Moreover, 
the accumulating particles in the nucleus might be degraded after a while, so that the steady state rRNA level might remain relatively stable. To address these questions and to analyze the onset of the defect, pulse-chase experiments should be conducted. Despite the slight variations in rRNA processing, the overall production of both ribosomal subunits is not altered in the $d b p 5$ mutants, as seen from the regular peak sizes in the ribosome profiles of Figure 21. In addition, the complete polysome profile of rat8-2 does also not show a reduction in the peak sizes of free $40 \mathrm{~S}$ and $60 \mathrm{~S}$ subunits compared to wild type cells (Figure 27A). Additionally, no other characteristics for biogenesis defects of only one ribosomal subunit, like the imbalance of the subunit peaks or the appearance of halfmeres (Li et al., 2009), is observed (Figure 27A). In conclusion, it remains unlikely that slight biogenesis defects lead to the strong ribosomal transport defects of the dbp5 mutants.

A direct involvement of Dbp5 in the transport of both pre-ribosomal subunits is more likely and could be corroborated by physical and genetic interactions of Dbp5 with established ribosomal transport factors (Figure 22 and Figure 23). So far several synthetic lethality screens were already performed and it was shown that Dbp5 genetically interacts with various mRNA transport and processing factors (Estruch and Cole, 2003; Estruch et al., 2012; Scarcelli et al., 2008; Snay-Hodge et al., 1998). However, in the present study genetic interactions between Dbp5 and the ribosomal export factors Mtr2 and Nmd3 (Figure 22) are shown for the first time and argue for their collaborative participation in ribosomal transport. Furthermore, Dbp5 interacts in vivo with the karyopherin Xpo1 and its adapter Nmd3 (Figure 23) showing that Dbp5 is able to physically contact the export factor-bound ribosomal particles. However, Dbp5 might also bind to Xpo1 independent of ribosomal transport, as it is suggested that Dbp5 molecules are actively exported from the nucleus by the karyopherin Xpo1 and the Ran GTPase cycle (Hodge et al., 1999). In fact, Dbp5 shuttles between nucleus and cytoplasm and accumulates in the nuclei of xpo1-1 cells and in mutants of the Ran GTPase cycle (Figure 24) (Hodge et al., 1999). However, Dbp5 does not contain a known NES and the complex formation between Dbp5 and Xpo1 was so far not shown in vitro. Thus, the underlying transport conditions for Dbp5 are still unclear. Nevertheless, an additional contact between Xpo1 and Dbp5 during ribosomal transport is well possible and the in vivo interaction of Dbp5 with the adapter protein Nmd3 (Figure 23) confirms that an association with the exported ribosomal particles is likely. However, how this binding is mediated cannot be judged from the co-immunoprecipitation experiments performed so far. Dbp5 as a transient 
RNA-binding protein (Weirich et al., 2006) might directly contact the rRNA of the ribosomal particles, but might also associate with ribosomal proteins or bound export factors. As shown in Figure 31, Dbp5 is able to directly bind the heterodimeric Mex67-Mtr2, which might also provide the contact to the exported ribosomal subunits. In vitro binding studies should be performed to identify the direct interaction partners.

Furthermore, the binding sites of the Dbp5 protein that contribute to the association with the exported ribosomal particles should be analyzed. As the intensity of the ribosomal mislocalization varies in the different dbp5 mutants (Figure 19), distinct domains of Dbp5 might be affected, which contribute differently to the transport of ribosomal particles. All analyzed dbp5 alleles contain point mutations that lead to single amino acid substitutions in the helicase core of Dbp5 (Figure 16) (Snay-Hodge et al., 1998). However, the entire structural and biochemical impacts of these amino acid exchanges are unknown, so that the consequences for the enzyme are difficult to estimate. It is possible that the complete structure of the RNA-helicase is altered, especially by the exchanges including the strand-breaking amino acid proline in rat8-7 and rat8-2. In fact, one salt bridge that is necessary for the interaction of Dbp5 with Nup159 is affected by the replacement of arginine by cysteine at position 256 of the rat8-1 protein (Noble et al., 2011; von Moeller et al., 2009). However, further structural consequences for the protein are well possible. All mutated proteins are not degraded upon shift to the restrictive temperatures (Snay-Hodge et al., 1998), but the rat8-2 protein is known to accumulate in cytoplasmic foci (Scarcelli et al., 2008). This effect might be comparable with a depletion of Dbp5 from the cytoplasmic fibrils of the NPC, which might lead to the ribosomal export defects. In conclusion, identification of important Dbp5 domains for ribosomal transport is crucial to completely understand its function, but needs further investigation.

In summary, Dbp5 is required for the nuclear export of both pre-ribosomal subunits and contacts the transported particles in vivo. However, Dbp5 does not accompany the pre-ribosomal subunits from the nucleus into the cytoplasm, as Dbp5 does not accumulate together with the ribosomal particles in the nucleus of mutants defective in ribosomal transport (Figure 24). In contrast, the nuclear retention of Dbp5 in xpo1-1 cells might rather be caused by the independent nuclear export of Dbp5 molecules via the karyopherin Xpo1 (Hodge et al., 1999). Thus, Dbp5 behaves differently than most of the established ribosomal export factors, which bind the preribosomal particles in the nucleus and support their translocation through the NPC by 
shielding these charged huge complexes from the hydrophobic interior of the NPC and by interaction with the FG-repeats (Gerhardy et al., 2014). Contrary, Dbp5 is located at the cytoplasmic fibrils of the NPC similar to mRNA export and might act rather shortly on the ribosomal particles upon their appearance in the cytoplasm (Figure 24 to Figure 26) (Hodge et al., 1999; Schmitt et al., 1999; Snay-Hodge et al., 1998; Tseng et al., 1998; Weirich et al., 2004). From there, Dbp5 might also contribute to a directional transport. However, the ATPase-dependent remodeling of RNA-protein complexes, which is characteristic for the DEAD-box RNA-helicase Dbp5 and necessary for the directional mRNA export (Lund and Guthrie, 2005; Tran et al., 2007), seems to be dispensable for its function in ribosomal transport.

\subsubsection{The ATPase-dependent RNP remodeling of Dbp5 is dispensable for ribosomal transport}

Lund and Guthrie (2005) found in dbp5 mutants an increased binding of the export receptor Mex67 to poly(A)-tail containing mRNAs at the nuclear rim and suggested that Dbp5 is required to dissociate Mex67 from the exported mRNAs. As Mex67 is additionally involved in the transport of both pre-ribosomal subunits (Faza et al., 2012; Yao et al., 2007), Dbp5 might also release Mex67 from the exported ribosomal complexes. However, a similar enhanced interaction of Mex67 with ribosomal proteins is not visible in the $d b p 5$ mutants (Figure 28). Moreover, the ribosomal association of Mex67 is not increased in sucrose-density gradients of rat8-2 cells treated with RNase A (Figure 27B). Therefore, these results suggest that the export receptor Mex67 is not displaced from the ribosomal subunits by Dbp5 and thus, Dbp5 behaves differently in ribosomal and mRNA transport.

In particular, Mex67 is even detectable in the polysomal fractions of sucrose-density gradients with wild type cells and this association is mediated by its binding to the translating ribosomes, as confirmed by the RNase A treatment of the lysates prior sucrose-density gradient fractionation (Figure 27). Thus, Mex67 can be part of actively translating ribosomes and is not dissociated from the ribosomal subunits before translation even in wild type cells. This is a new finding and contrary to the results of Windgassen et al. (2004), which showed only a marginal association of Mex67 with polysomes. However, they used for the detection in the Western blot analyses a tagged version of the protein, which was expressed from a plasmid in wild type cells. Thus, the tagged protein might behave differently than the endogenous. Furthermore, the overexpression resulting from an extra copy of MEX67 might 
influence the localization of Mex67. In contrast to their experiments, a specific antiMex67 antibody was used for the detection of endogenous Mex67 in the studies shown in Figure 27. Thus, these differences might explain the varying polysomal localization of Mex67. Whether Mex67 might have a function in the translation process or whether Mex67 is simply recycled from the ribosomal subunits during translation and thus, later than expected (Gerhardy et al., 2014), are exciting questions, which should be analyzed in future studies. However, Mex67 could also be released from the ribosomal subunits during the cytoplasmic maturation and re-associate with the ribosomes during translation.

Regardless of where the recycling takes place, Dbp5 is generally not required for the displacement of Mex67 from the ribosomal particles, because the ribosomal association of Mex67 is not increased in dbp5 mutants (Figure 27 and Figure 28). Neither the interaction of Mex67 with ribosomal proteins is enhanced in coimmunoprecipitation studies (Figure 27), nor is its amount in free ribosomal subunits or 805 ribosomes-containing fractions of sucrose-density gradients (Figure 28) enlarged in dbp5 mutants. Moreover, the increased polysomal localization of Mex67 in sucrose-density gradients of rat8-2 cells (Figure 27A) can be explained by its enhanced binding to the translated mRNAs, as upon RNase A treatment the association with the $80 \mathrm{~S}$ ribosomes is not altered compared to the wild type (Figure 27B). In detail, Mex67 associated with the polysomal fractions can be bound to the translating ribosomes or to the translated mRNA. However, the RNase A degrades the single-stranded mRNAs so that all ribosomes form one $80 \mathrm{~S}$ peak, whose fractions contain all ribosome-bound Mex67 proteins and their amount is unchanged in rat8-2 cells (Figure 27B). Thus, these data suggest that different pools of Mex67 molecules exist. The Mex67 molecules that bind to the mRNA are displaced upon export by Dbp5 in wild type cells and remain mRNA bound in rat8-2 cells (Lund and Guthrie, 2005). Therefore, the few mRNAs that can emerge in the cytoplasm and can be translated in rat8-2 cells should carry more Mex67 molecules leading to the increased polysomal association of Mex67 despite the reduced translation rate (Figure 27A). In contrast, the other pool of Mex67 molecules binds to the ribosomal subunits and these molecules are not dissociated by Dbp5. Thus, they can associate with the translating ribosomes to a similar extent in rat8-2 and wild type cells (Figure 27B).

Summarizing, Dbp5 acts differently on Mex67 molecules that are bound to the ribosomal particles or to the mRNAs. The question is how Dbp5 can discriminate 
between these molecules. The major difference is that Mex67 binds directly via specific loops to the rRNA of the pre-ribosomal subunits (Figure 3) (Yao et al., 2007), whereas the interaction to the mRNA is mainly mediated by adapter proteins such as Npl3, Gbp2, Hrb1, Yra1 or Nab2 (Hackmann et al., 2014; Kelly and Corbett, 2009). Thus, Dbp5 might not be able to dissociate Mex67 molecules that are directly bound to RNAs. Possibly, the displacement of Mex67 from the mRNA might require the concomitant release of its adapter protein Nab2, which was already confirmed as a target of Dbp5's remodeling activity in vitro (Tran et al., 2007). However, further studies will be needed to completely answer this question.

In conclusion, Dbp5 does not dissociate the export receptor Mex67 from ribosomal particles. Nevertheless, the RNP remodeling activity of the DEAD-box RNA-helicase might be needed for the release of other bound ribosomal export factors such as Nmd3 or Xpo1. However, the recycling pathways of several established export factors are already known (Figure 4) (Gerhardy et al., 2014) and thus, an additional involvement of Dbp5 is possible, but unlikely. In particular, Xpo1 is released immediately upon arrival in the cytoplasm by the Ran GTPase cycle (Fornerod et al., 1997; Stade et al., 1997), whereas its adapter $\mathrm{Nmd3}$ is recycled in the last cytoplasmic maturation step by Lsg1 (Hedges et al., 2005). Furthermore, the release of Arx1 depends on Rei1 together with Jjj1 and the ATPase Ssa1/Ssa2 (Gerhardy et al., 2014). Npl3 remains bound until translation and its release from the polysomes depends on Mtr10 (Windgassen et al., 2004). In addition, factors such as Ecm1 and Arx1 are non-essential (Bradatsch et al., 2007) and therefore, it is unlikely that their missing recycling leads to the strong ribosomal export defects seen in the $d b p 5$ mutants. Moreover, most of these factors mediate especially the transport of the pre$60 S$ subunit, but the pre-40S export is also affected in the $d b p 5$ mutants.

Finally, the ATPase-dependent RNP remodeling activity of Dbp5 in general seems not to be required for efficient ribosomal transport, as the regulated ATPase cycle of Dbp5 is dispensable for this process (Figure 29 and Figure 30). The ATPase activity of Dbp5 is stimulated by the co-factors Gle1 and IP 6 (Alcazar-Roman et al., 2006; Weirich et al., 2006). However, only very faint ribosomal transport defects are visible in the temperature-sensitive mutant gle1-4 (Figure 29) in accordance with previously published results from ribosomal export screens (Moy and Silver, 1999; StageZimmermann et al., 2000). These data indicate that the Dbp5 stimulation by Gle1 is not necessary for ribosomal transport. Nevertheless, another unknown protein could stimulate the ATPase activity of Dbp5 during ribosomal transport. Thus, dbp5 
mutants that are unable to hydrolyze ATP were tested. Overexpression of dominantnegative alleles of ATPase-deficient dbp5 mutants only affect mRNA (Hodge et al., 2011), but not ribosomal export of wild type cells (Figure 30) suggesting that the ATPase-activity in general is dispensable for ribosomal transport. The detailed mechanism underlying the dominant-negative phenotype of $d b p 5-R 426 Q$ is not clear yet, but the mutant protein exhibits a reduced ATP- and RNA-binding affinity accompanied by a minimal ATPase activity (Hodge et al., 2011). In the second ATPase-deficient dbp5 mutant, the dbp5-R369G protein has a remaining ATPase activity of $60 \%$ and is anticipated to compete with the wild type Dbp5 for Gle1 stimulation that leads to the observed mRNA export defects (Hodge et al., 2011). Thus, these results confirm that the Gle1-mediated activation of the ATP-hydrolysis by Dbp5, which is a prerequisite for the protein displacement during mRNA export (Tran et al., 2007), is indeed not essential for the role of Dbp5 during ribosomal transport.

In contrast to Gle1, the nucleoporin Nup159, which also regulates the ATPase cycle of Dbp5, seems to be involved in the ribosomal export process. Indeed, the rat7-1/nup159 mutant shows slight nuclear accumulations of both pre-ribosomal subunits (Figure 26), as already described (Gleizes et al., 2001; Moy and Silver, 2002; Stage-Zimmermann et al., 2000). On the one hand, the mild ribosomal export defects might indicate that the nucleoporin Nup159 is needed to localize Dbp5 to the cytoplasmic fibrils of the NPC for efficient ribosomal transport similar to mRNA export (Hodge et al., 1999; Schmitt et al., 1999; Weirich et al., 2004). On the other hand, it is possible that Nup159 is required for ribosomal transport on its own and independent of Dbp5, as suggested by Gleizes et al. (2001). The authors showed pre-40S transport defects at $34^{\circ} \mathrm{C}$ for rat7-1/nup159, but not for rat8-2 and nup $159 \Delta \mathrm{N}$ lacking the interaction domain for Dbp5. The rat7-1/nup159 allele creates a premature termination codon, which leads to the truncation of the protein at the C-terminus and its missing anchoring within the NPC (Del Priore et al., 1997). Thus, the authors concluded that the C-terminus of Nup159 might be sufficient to facilitate efficient pre$40 \mathrm{~S}$ export independent of Dbp5, as the integrity of the NPC is maintained (Gleizes et al., 2001). However, the rather mild temperature shift to $34^{\circ} \mathrm{C}$ might not be sufficient to induce the ribosomal transport defects in rat8-2 and nup159 $\Delta N$. Furthermore, the authors did not analyze pre-60S export. Thus, further experiments would be needed to confirm a possible Dbp5-independent function of Nup159 in the nuclear export of both pre-ribosomal subunits. 
During nuclear mRNA export, two functions on Dbp5 are described for Nup159. Firstly, Nup159 is suggested to be the ADP-release factor of Dbp5 and leads to the recycling of the enzyme for a new ATPase cycle (Noble et al., 2011). Secondly, Nup159 as part of the cytoplasmic filaments interacts with Dbp5 via its N-terminal domain that contributes to the tethering of Dbp5 to the cytoplasmic side of the NPC (Hodge et al., 1999; Schmitt et al., 1999; Weirich et al., 2004). Thus, the proper localization of recycled Dbp5 for its role in mRNA export is archived by Nup159. In rat7-1/nup159 cells, these Dbp5 molecules are detached from the nuclear rim (Hodge et al., 1999) and consequently, the mRNAs accumulate strongly and rapidly in the nucleus (Figure 26C) (Gorsch et al., 1995). Thus, the lack of Dbp5 at the cytoplasmic fibrils might also cause the nuclear accumulation of pre-ribosomal particles in rat7-1/nup159 cells (Figure 26). As the ribosomal transport is less affected than the mRNA export in this strain (Figure 26), Nup159 might simply be needed for tethering Dbp5 to the cytoplasmic side of the NPC and stimulation of the ADP-release of Dbp5 might be dispensable for efficient ribosomal export. This possibility is especially corroborated by the results showing that the stimulation of the ATP-hydrolysis of Dbp5 is also not necessary for ribosomal transport (Figure 29 and Figure 30). To confirm the importance of the interaction between Dbp5 and Nup159 for ribosomal export, dbp5 mutants defective in Nup159 binding like dbp5R256D/R259D (Noble et al., 2011) should be analyzed for ribosomal transport defects in future experiments.

In fact, Gle1 bound to the cytoplasmic nucleoporin Nup42/Rip1 contributes as well to the NPC localization of Dbp5, as Dbp5 alternately interacts with Gle1 and Nup159 during its ATPase cycle (Hodge et al., 2011; Murphy and Wente, 1996; Strahm et al., 1999). However, the Dbp5 localization is not substantially altered in gle1 mutants like gle1-37 (Hodge et al., 1999; Strahm et al., 1999) and although not tested yet, Dbp5 might behave similarly in gle1-4 cells. Thus, enough Dbp5 molecules might be available at the cytoplasmic fibrils for its function in ribosomal transport, so that no severe ribosomal export defects are caused in gle1-4 cells (Figure 29).

In conclusion, these results suggest that the regulated ATPase cycle of Dbp5 in general is dispensable for the nuclear export of pre-ribosomal subunits, but its localization at the cytoplasmic fibrils seems to be crucial for this process. Thus, it seems likely that Dbp5 does not remodel the pre-ribosomal particles upon their cytoplasmic appearance contrary to the exported mRNPs indicating differences in the transfer mode of these two large RNA-protein complexes. 


\subsubsection{How might Dbp5 contribute to the nuclear export of ribosomal particles?}

Therefore, if Dbp5 does not displace transport factors from the emerging preribosomal subunits at the cytoplasmic side of the NPC, the question is how Dbp5 contributes to the directional ribosomal transport.

Placed at the cytoplasmic fibrils, Dbp5 might contact the exported ribosomal particles rather shortly. Indeed, Dbp5 seems to act on the pre-60S subunits before the first cytoplasmic maturation step, which is performed by Drg1 (Kappel et al., 2012; Pertschy et al., 2007), because Dbp5 does not accumulate on Nmd3-containing immature pre-60S subunits in the drg1-18 mutant (Figure 25B). In detail, the export adapter protein Nmd3 cannot be recycled from the pre-60S subunits and accumulates on these immature particles in the cytoplasm of drg1-18 cells (Bassler et al., 2012; Kappel et al., 2012). Thus, an enhanced interaction between Nmd3 and Dbp5 would be expected, if both proteins would accumulate together on the aberrant pre-60S subunits, but this effect is not visible (Figure 25B). Therefore, it remains unlikely that Dbp5 is loaded onto the exported pre-ribosomal subunits at the cytoplasmic side of the NPC and stays bound or is recycled during the cytoplasmic biogenesis. In contrast, the interaction between Mex67 and Arx1-containing particles is enhanced in the drg1-18 mutant (Figure 25A). This result suggests that indeed aberrant pre-60S subunits with the bound export factors Arx1 and Mex67 accumulate in the cytoplasm in this mutant, as reported earlier by Kappel et al. (2012). However, given that Mex67 remains bound to the ribosomal subunits until translation (see section 6.1.5), this stronger interaction derives rather from an increased binding of Arx1 to the Mex67-bound ribosomal particles and is rather caused by the missing recycling of Arx1.

In summary, these data suggest that Dbp5 bound to the cytoplasmic filaments might act on the pre-ribosomal particles prior to the cytoplasmic maturation. Placed there, Dbp5 might transiently contact export factors bound to the pre-ribosomal subunits. Indeed, Dbp5 is able to interact in vivo and in vitro with the export receptor Mex67 (Figure 31), which transports mRNAs and both, the pre-40S and pre-60S subunits (Faza et al., 2012; Segref et al., 1997; Yao et al., 2007). As expected for two mRNA export factors and already reported by Lund and Guthrie (2005), the in vivo binding between Mex67 and Dbp5 is greatly RNA-dependent (Figure 31A). Most likely, both proteins can be bound to the same mRNA molecule during transport. Additionally, a sub-fraction of Dbp5 and Mex67 molecules is able to interact RNA-independently and directly with each other (Figure 31). Certainly, further studies are needed to localize 
this interaction in the cell. However, it is well possible that this direct contact transiently occurs during ribosomal transport.

Thus, Dbp5 at the cytoplasmic side of the NPC might shield the export receptor Mex67 from its interaction with the FG-repeat containing nucleoporins of the NPC channels. Consequently, Dbp5 might prevent the back-sliding of the exported particles and in this way, supports Xpo1 and the Ran GTPase cycle by enabling the directional ribosomal transport. To confirm such a model, it should be demonstrated that Dbp5 is indeed able to reduce the binding of Mex67 to the FG-repeats. Furthermore, Dbp5 might contact and shield additional ribosomal export factors that should be analyzed by in vitro binding studies. Moreover, it is unknown which conformation and which domain of Dbp5 could contribute to the binding of the exported pre-ribosomal particles. As the ATPase cycle of Dbp5 is dispensable for ribosomal transport (Figure 29 and Figure 30) and Nup159-bound Dbp5 excludes RNA binding (Montpetit et al., 2011; von Moeller et al., 2009), a protein contact might be established by the surface of Dbp5 independent of its nucleotide-binding. Thus, ADP-Dbp5 bound to Nup159 or ATP-Dbp5 bound to Nup42 via Gle1 (see ATPase cycle Figure 13) could contribute equally to the association with the ribosomal particles from the cytoplasmic fibrils.

In conclusion, the exact mechanism of how Dbp5 facilitates the ribosomal transport needs further investigations, however, the present study confirms that Dbp5 is required for this process and acts differently than during mRNA export. 

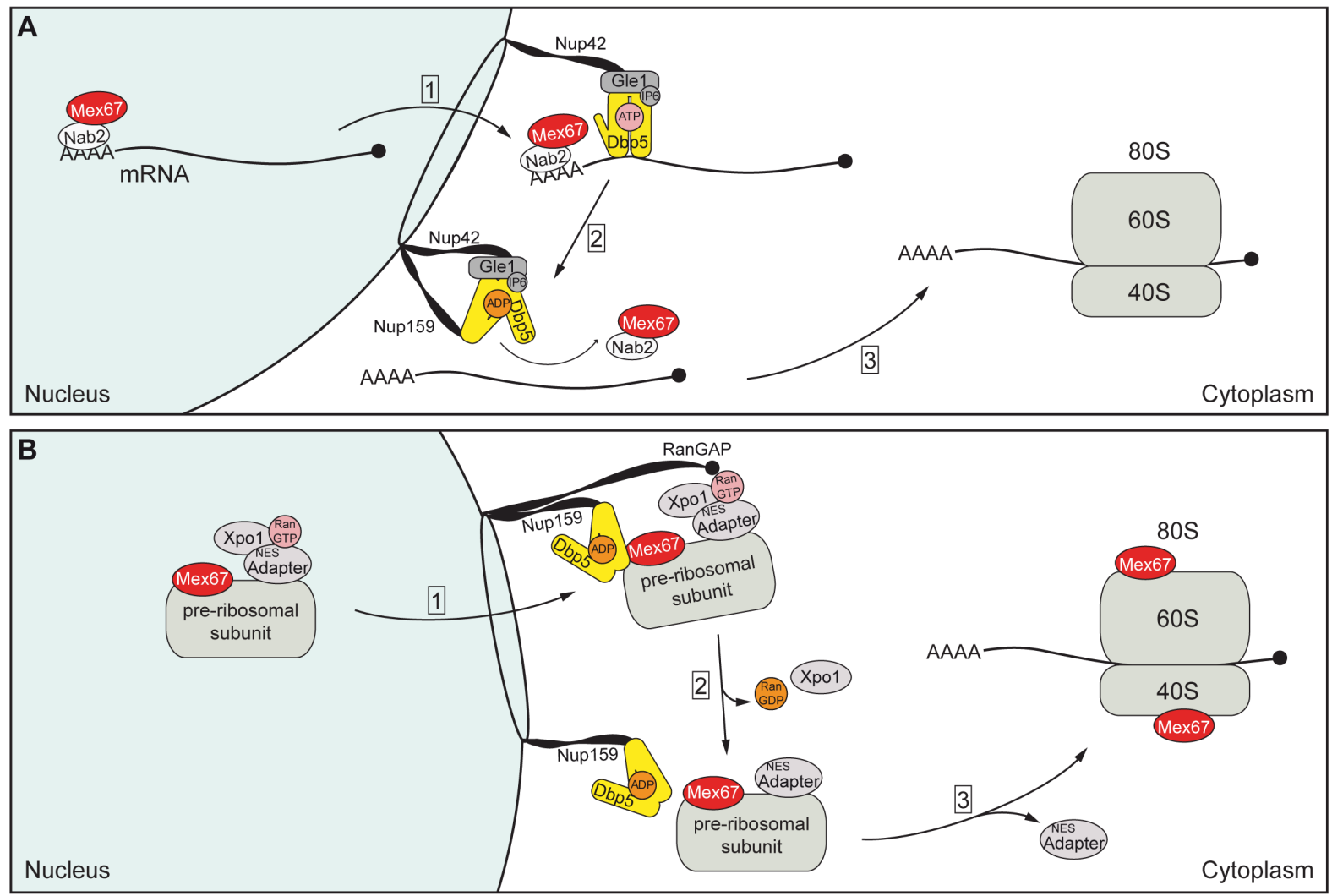

Figure 39: Model for the differences in Dbp5-mediated nuclear export of mRNAs and preribosomal subunits.

(A) The role of Dbp5 in nuclear mRNA export is schematically shown. The export receptor Mex67, which is bound to adapter proteins such as Nab2, facilitates the translocation of nuclear mRNAs into the cytoplasm (1). ATP-Dbp5 localized to the cytoplasmic fibrils binds the exported mRNAs, which facilitates the $\mathrm{IP}_{6}$ and Gle1-stimulated ATP-hydrolysis. The transition into ADP-Dbp5 leads to RNA release and the concomitant displacement of Nab2 and Mex67 (2). The remodeled mRNPs remain in the cytoplasm and can be translated (3). Afterwards, Nup159 triggers the ADP-release of Dbp5 and the recycling of the enzyme. (B) Model of ribosomal export mediated by Dbp5. Both preribosomal subunits bind directly Mex67 and the karyopherin Xpo1 via NES-containing adapter proteins (Nmd3 for pre-60S, unknown for pre-40S), which facilitate translocation through the NPC. Dbp5 placed at the cytoplasmic fibrils acts ATPase independently and transiently contacts the ribosomal particles possibly via Mex67 and might prevent their back-sliding. GTP-hydrolysis by Ran leads to the dissociation of Xpo1 and RanGDP and to the directional ribosomal transport (2). During cytoplasmic maturation all transport factors are recycled except for Mex67, which remains bound until translation (3).

Even though Dbp5 seems to act in both transport processes at the cytoplasmic fibrils of the NPCs, the need for its enzymatic activity as an RNA-helicase, which is able to remodel RNA-protein complexes, is variable. The displacement of bound transport factors such as Mex67 from the emerging mRNAs is a prerequisite of the directional mRNA export and requires the ATPase cycle of Dbp5 regulated by Nup159 and Gle1/IP 6 (Figure 39A). In contrast, a Dbp5-mediated remodeling of exported preribosomal subunits does not take place and the ATPase cycle of Dbp5 in general is dispensable for ribosomal transport (Figure 39B). Thus, Mex67 remains bound to the ribosomal subunits. Instead, solely the contact of Dbp5 to the emerging preribosomal subunits at the cytoplasmic side of the NPCs might support their fast and 
directional translocation. In conclusion, distinct transport mechanisms of Dbp5 for the transfer of different large ribonucleoprotein particles must exist.

Thus, the results of the present study add a new member to the large network of ribosomal transport factors, which is relevant for the general understanding of the export processes for both pre-ribosomal subunits. Additional insights into the underlying export mechanisms are especially important for the pre-40S transport, which is barely understood.

Remarkably, the present study also shows that Mex67 is associated with actively translating ribosomes of wild type cells. This unexpected and novel result suggests that the export receptor Mex67 might either be recycled later as so far expected from the ribosomal subunits during translation or might even have an additional function in the translation process. Both possibilities would influence the current understanding of Mex67 and its functionality and thus, should be analyzed in future studies.

Moreover, the finding that Dbp5 is required for the nuclear export of pre-ribosomal subunits will also impact the studies on its cytoplasmic function in translation termination.

\subsection{Dbp5 and Rli1 interact during translation termination}

In addition to its function in nuclear export events, the DEAD-box RNA-helicase Dbp5 and its ATPase activity is also required for translation termination in the cytoplasm, as reported by Gross et al. (2007). The authors showed that Dbp5 is part of actively translating ribosomes and interacts genetically and physically with the canonical eukaryotic release factors eRF1 and eRF3. Moreover, temperature-sensitive $d b p 5$ mutants are hypersensitive to translational inhibitors and reveal an increased readthrough rate of stop codons (Gross et al., 2007). Similar results were obtained for the co-factor Gle1, which is also needed for translation termination by stimulation of the ATPase activity of Dbp5 in concert with $\mathrm{IP}_{6}$ (Alcazar-Roman et al., 2010; Bolger et al., 2008). Furthermore, the iron-sulfur containing ABC-family ATPase Rli1 was characterized as third additional translation termination factor, whose depletion leads to an increased stop codon read-though activity (Khoshnevis et al., 2010). However, the underlying mechanism is still discussed. Shoemaker and Green (2011) and Preis et al. (2014) suggested that Rli1 contributes ATP-independently to the termination process by stabilizing eRF1 in a favorable conformation for peptidyl-tRNA hydrolysis. 
According to these data, Dbp5, Gle1 and Rli1 are necessary in addition to eRF1 and eRF3 to mediate efficient translation termination. However, a model that provides a chronological order of events including all of these factors is still missing. Thus, the second part of the present study aimed to characterize the translation termination complex and especially, to analyze the recruitment of Dbp5 and Rli1 to this complex.

\subsubsection{Dbp5, its co-factor Gle1 and Rli1 interact with each other during translation termination}

To analyze the formation of the termination complex in vivo, several coimmunoprecipitation experiments were performed. These studies revealed that Dbp5 and its co-factor Gle1 bind RNA-independently to Rli1-containing complexes (Figure 32). As complete yeast cell lysates were used in these assays, the association of these proteins might be indirect, but could be mediated by larger protein complexes like the translation machinery. Thus, it is possible that Dbp5, Gle1 and Rli1 simultaneously bind to the same ribosome, which might take place during translation termination. However, they might also contact each other during other cellular processes such as the ribosomal transport. To distinguish between these possibilities, the interaction between Dbp5, Gle1 and Rli1 was analyzed under conditions that prevent the proceeding of translation termination (Figure 33 and Figure 34).

Indeed, less Dbp5 and Gle1 molecules associate with Rli1-containing particles in the eRF1 mutant sup45-2 (Figure 33). As the mutant protein sup45-2 fails to associate with ribosomes and consequently disturbs stop codon recognition (Stansfield et al., 1997), these data indicate that the interaction between Dbp5, Gle1 and Rli1 depends on the formation of the translation termination complex by eRF1. However, the results regarding Gle1 should be analyzed carefully, because myc-Gle1 was in general difficult to detect in the Western blot analyses (Figure 32 and Figure 33). Possibly, addition of further myc-tags might enhance the signals, so that the experiments can be repeated to obtain significant results. According to these difficulties with Gle1, the present study is focusing on Rli1 and Dbp5.

Blocking translation elongation and preventing subsequent translation termination by cycloheximide treatment also leads to a slightly reduced association of Dbp5 and Rli1 (Figure 34). These data suggest that both proteins indeed interact during translation termination most likely within the termination complex. Certainly, the interaction between Dbp5 and Rli1 was in none of these experiments completely prevented 
(Figure 33 and Figure 34). One reason could be that the used cycloheximide concentration was not sufficient to block the elongation process completely, so that translation termination events could still take place. An increased concentration was able to further decrease the interaction between Rli1 and Dbp5, as an initial experiment already showed (data not shown). Therefore, further experiments with varying conditions of this antibiotic should be performed. Furthermore, the remaining interaction between Rli1 and Dbp5 might originate from an additional contact during another cellular process such as the ribosomal transport. Rli1 is involved in the nuclear export of pre-ribosomal particles (Kispal et al., 2005; Yarunin et al., 2005), for which Dbp5 is also required, as now shown in section 6.1. Thus, Dbp5 might additionally associate with Rli1-containg pre-ribosomal subunits during their transport. This explanation seems possible, as this contact would not be altered by the inhibition of translation termination. However, a simultaneous function of Rli1 and Dbp5 in the ribosomal transport remains to be shown.

In summary, these results show that at least a sub-fraction of Dbp5 molecules, possibly together with Gle1, interacts with Rli1 during translation termination. These in vivo interactions are novel findings, which might suggest a simultaneous binding of Dbp5, Gle1 and Rli1 to the terminating ribosome at least during one step of the termination process.

Interestingly, a contact formation between these three proteins during translation termination contradicts current termination models. The DEAD-box RNA-helicase Dbp5 and its co-factor Gle1 are believed to act rather early during this process (Baierlein and Krebber, 2010; Bolger et al., 2008; Gross et al., 2007). Dbp5 might enter together with eRF1 the ribosomal A-site harboring a stop codon and Gle1- and $\mathrm{IP}_{6}$-dependent stimulation of its ATPase-activity might lead to the remodeling of the ribosomal complex, which subsequently mediates the binding of eRF3. According to this model, dissociation of Dbp5 and Gle1 is a prerequisite for the entry of eRF3 to the termination complex (Bolger et al., 2008; Gross et al., 2007). In contrast, the ABC-family ATPase Rli1 is implicated to be required during later steps of the termination process (Becker et al., 2012; Preis et al., 2014; Shoemaker and Green, 2011). Biochemical studies suggested that Rli1 functions ATP-independently in translation termination upon the GTP-hydrolysis and dissociation of eRF3 (Shoemaker and Green, 2011). Furthermore, structural analyses confirmed that eRF3 and Rli1 share the binding site on the terminating ribosome indicating a mutually exclusive binding of both termination factors (Preis et al., 2014). Thus, Rli1 
might bind upon eRF3-GDP dissociation to the termination complex and might stabilize eRF1 in an optimal position for the peptidyl-tRNA hydrolysis (Preis et al., 2014; Shoemaker and Green, 2011). According to these models, a contact between Rli1 and Dbp5 together with Gle1 would not happen during translation termination. Thus, the results from the present work (Figure 32 to Figure 34) suggest that Rli1 might be recruited earlier to the terminating ribosomes as so far expected so that an interaction between these termination factors would be possible. Alternatively, Dbp5 with Gle1 might act later during the termination process when Rli1 is also needed. However, Dbp5 and Gle1 interact only RNA-mediated with eRF3 in vivo (Bolger et al., 2008; Gross et al., 2007) so that a simultaneous binding to the terminating ribosomes seems to be not possible. Nevertheless, Dbp5 and Gle1 recruitment upon eRF3 dissociation would be an explanation. However, the interaction between eRF1 and eRF3 was decreased in dbp5 mutants (Gross et al., 2007) and less eRF3 was associated with polysomes in dbp5 and gle1 mutants (Bolger et al., 2008; Gross et al., 2007). These results supported the idea that the activity of Dbp5 and its stimulation by Gle1 are necessary for the efficient incorporation of eRF3 into the termination complex and that the eRF3 entry occurs upon dissociation of Dbp5 and Gle1.

To answer the question when Dbp5 and Rli1 interact during translation termination, the recruitment of Dbp5 and Rli1 to the ribosomes was analyzed.

\subsubsection{Dbp5 is recruited to the ribosomes during translation}

In co-immunoprecipitation studies, Dbp5 is less associated with small and large ribosomal proteins in the eRF1 mutant sup45-2 (Figure 35). These results indicate that functional eRF1 is important to recruit Dbp5 to ribosomal complexes. Thus, Dbp5 might indeed join together with eRF1 the ribosomes harboring a stop codon in their ribosomal A-site, as already suggested by Gross et al. (2007). However, from these experiments with complete yeast cell lysates, the ribosomal particles, to which Dbp5 associates, are not distinguishable. These particles might be $80 \mathrm{~S}$ ribosomes or free ribosomal subunits. Therefore, sucrose-density gradient analyses were performed to discriminate the different ribosomal species. As seen in Figure 37, Dbp5 is particularly decreased in the fractions containing single 805 ribosomes (monosomes), but also slightly reduced in the polysomal fractions of sup45-2 cells, whereas the association with free ribosomal subunits is not altered compered to wild type cells. First of all, these results show that Dbp5 is still able to interact with free 
$40 \mathrm{~S}$ and $60 \mathrm{~S}$ subunits independent of eRF1. This contact most likely occurs during the nuclear export of pre-ribosomal subunits (see section 6.1), which should not be disturbed by inhibition of translation termination. Thus, the remaining interaction of Dbp5 with the ribosomal proteins Rps3 and Rpl35 might result from its binding to the pre-ribosomal subunits (Figure 35).

Furthermore, the reduced association of Dbp5 with ribosomes in the sucrose-density gradient of sup45-2 cells (Figure 37) confirms that Dbp5 interacts eRF1-mediated with $80 \mathrm{~S}$ ribosomes. Thus, eRF1 might indeed be necessary for the recruitment of Dbp5 to the terminating ribosomes. However, the sup45-2 strain is also slightly defective in protein synthesis, as already published by Stansfield et al. (1997) and as also visible in the polysome profile (Figure 36). The polysome run-off and the concomitantly increased $80 \mathrm{~S}$ peak indicate a slightly reduced translation rate in sup45-2 cells and are characteristic for cells defective in translation initiation (Masek et al., 2011). In these cells, fewer ribosomes are engaged in translation leading to the reduced polysomes and more mRNA-free $80 \mathrm{~S}$ ribosomes, so called couples exist, which form the enlarged $80 \mathrm{~S}$ peak. Thus, the lack of functional eRF1 and a decreased translation termination rate might indirectly affect the translation efficiency. Alternatively, eRF1 might not only be important for translation termination, but also be needed for efficient translation initiation. This question must be answered in future studies.

In summary, an eRF1 mediated recruitment of Dbp5 to terminating ribosomes is possible, especially as the amount of Dbp5 in the polysomal fractions of sup45-2 cells is slightly reduced. Nevertheless, an earlier recruitment during translation initiation and/or elongation cannot be ruled out. As the translation rate in general is affected in sup45-2, Dbp5 could also be loaded onto the ribosomes during any step of the translation process and might to wait for the arrival at the stop codon and the entry of eRF1. To distinguish between these possibilities, the ribosomal association of Dbp5 should also be analyzed in mutants of initiation and elongation factors. However, as Dbp5 revealed no interaction with initiation factors so far (Bolger et al., 2008), recruitment during translation initiation remains unlikely.

Furthermore, considering that the $80 \mathrm{~S}$ peak is enlarged in sup45-2 compared to wild type cells, the association of Dbp5 with the monosome-containing fractions is particularly decreased in sup45-2 (Figure 36 and Figure 37). This peak should be predominantly formed by the accumulating mRNA-free 805 couples. Thus, the reduced binding of Dbp5 to these couples indicates that non-translating ribosomes 
do not carry Dbp5 molecules and that Dbp5 is indeed recruited to the ribosomes during the translation process. Therefore, it seems unlikely that Dbp5 is loaded onto pre-ribosomal subunits at the NPC and is transferred to its function in translation termination, as already suggested from the results of Figure 25 . In summary, the exact time point for the binding of Dbp5 to the terminating ribosomes could not be solved so far. However, the ribosomal recruitment must happen during the translation process and is most likely mediated by eRF1.

Thus, Dbp5 might indeed enter together with eRF1 the stop codon-containing ribosomal A-site, whereas eRF3 might only be recruited upon dissociation of Dbp5, as suggested previously (Baierlein and Krebber, 2010; Bolger et al., 2008; Gross et al., 2007). However, the separated recruitment of eRF1 and eRF3 to the ribosome contradicts current termination models, which anticipate a simultaneous ribosomal entry as the ternary complex eRF1-eRF3-GTP (Alkalaeva et al., 2006; Jackson et al., 2012; Shoemaker and Green, 2011). This assumption is especially corroborated by the strong interaction between eRF1 and eRF3 (Stansfield et al., 1995; Zhouravleva et al., 1995). Furthermore, structural analyses from Cheng et al. (2009) suggested that eRF1 in a complex with eRF3 has a higher affinity to the ribosome than sole eRF1. However, kinetic analyses that support this model are still missing. Moreover, Salas-Marco and Bedwell (2004) showed no alteration in the ribosomal binding of eRF1 in eRF3-depleted yeast cells indicating that the association with eRF3 is not a prerequisite to deliver eRF1 to the ribosome. Thus, eRF1 bound to the ribosomal Asite might also await the entry of eRF3, which might be mediated by Dbp5. In such a model, eRF3 should enter the ribosome in its GDP-bound form, as the affinity of eRF3 for GTP is only increased by its association with eRF1 (Pisareva et al., 2006). However, further investigations are needed to support such a model. For example, the potential mutually exclusive eRF1 binding of Dbp5 and eRF3 could be analyzed by performing in vitro binding studies and competition assays with purified factors.

\subsubsection{Is Rli1 eRF1-independetly recruited to the ribosomes?}

In contrast to Dbp5, the interaction of Rli1 with the ribosomal proteins of both, the $60 \mathrm{~S}$ and $40 \mathrm{~S}$ subunits, is not decreased in sup45-2 cells (Figure 38) that might argue for different ways of the recruitment of Dbp5 and Rli1 to ribosomal particles. In particular, the association of Rli1 with the large ribosomal protein Rpl35 representing the 605 subunits is not significantly altered in sup45-2 cells and rather an increased binding of Rli1 to the small ribosomal protein Rps3 is detectable (Figure 38). These 
results suggest that Rli1 is able to interact with the ribosomal subunits independent of functional eRF1. Indeed, Rli1 can also be recruited to ribosomes by Dom34 to mediate the recycling of ribosomal subunits during no-go decay (Pisareva et al., 2011; Shoemaker and Green, 2011) and the quality control of 80S-like ribosomes (Strunk et al., 2012). Thus, it is not expected that the ribosomal association of Rli1 is completely abolished in the eRF1 mutant sup45-2.

However, the non-decreased interaction between Rli1 and the ribosomal proteins (Figure 38) suggests that Rli1 might be already bound to the $80 \mathrm{~S}$ ribosomes during translation elongation and might await the arrival at the stop codon. Thus, its ribosomal association would not be changed, when translation termination does not occur. This possibility is corroborated by the unchanged interaction between Rps3 and Rli1 in the presence of cycloheximide (Figure 34). Thus, inhibition of elongation does not prevent the association of Rli1 with Rps3-containing 40S subunits. As already known, Rli1 also associates with 40S subunits during translation initiation, where it stabilizes the pre-initiation complex (Dong et al., 2004). Therefore, it is possible that upon initiation Rli1 remains bound to the 405 subunits and the subsequently formed $80 \mathrm{~S}$ ribosomes, until these reach the stop codons. The even increased binding of Rli1 to Rps3 (Figure 38) might therefore simply be explained by the translational defects seen in sup45-2 (Figure 36). A delayed initiation might lead to an increased accumulation of Rli1 on $40 \mathrm{~S}$ subunits in pre-initiation complexes.

However, the slightly increased interaction of Rli1 with eRF1-containing particles in the presence of cycloheximide (Figure 34) might argue for an additional contact of both proteins prior to translation termination. If both proteins interact during translation termination at the ribosome, a decreased association would be expected without proceeding of translation termination. Thus, unbound Rli1 and eRF1 might also interact in the cytoplasm prior to the ribosomal recruitment. However, a contact could also happen somewhere else, possibly during recycling events (Shoemaker and Green, 2011).

In summary, Rli1 might be recruited to the ribosomes earlier as expected prior to the formation of the termination complex. As the binding site at the terminating ribosome overlaps with the binding site of eRF3 (Preis et al., 2014), Rli1 might change its position on the $40 \mathrm{~S}$ subunit during this process. Possibly, Rli1 might be loosely associated with the $40 \mathrm{~S}$ subunit during translation initiation and elongation, so that Rli1 might be fast available for the termination reaction to take over the position of eRF3 upon its dissociation. This model would also explain the weak in vivo 
interaction of Rli1 with eRF3 (Khoshnevis et al., 2010). This interaction might be mediated by the ribosome and would not be possible, if Rli1 would enter the terminating ribosome after dissociation of eRF3. Furthermore, if Rli1 is loosely associated with translating ribosomes in a nucleotide-free conformation, this model would also be in accordance with data from in vitro experiments with recombinant Rli1. Pisarev et al. (2010) detected a weak in vitro interaction of nucleotide-free ABCE1/Rli1 with the ribosomal subunits and $80 \mathrm{~S}$ ribosomes, whereas upon ATPhydrolysis ABCE1/Rli1-ADP did not bind to any ribosomal particle. Furthermore, addition of the non-hydrolysable ATP analog AMPPNP leads to its enhanced association with 40S, 43S and eRF1-containing termination complexes, but not with release factor-free $80 \mathrm{~S}$ ribosomes (Pisarev et al., 2010). Therefore, Rli1 might be recruited to elongating ribosomes in its nucleotide-free conformation. Subsequently, upon arrival at the stop codon and eRF1 entry, the ATP-binding of Rli1 might occur that enhances its affinity for the terminating ribosome. Furthermore, addition of GMPPNP-bound eRF3 decreases the binding of ABCE1/Rli1 to eRF1-containing ribosomes (Pisarev et al., 2010) suggesting that the ATP-binding and high affinity association to the termination complex follows the dissociation of GDP-bound eRF3. Thus, the acquisition of the position of eRF3 by Rli1 might cause its increased ribosomal binding that is consistent with the structural data (Preis et al., 2014).

Further studies will be needed to confirm a model, in which nucleotide-free Rli1 is already loosely bound to elongating ribosomes awaiting the stop codon. However, this early ribosomal recruitment would also explain the observed in vivo interactions of Rli1 with Dbp5 and Gle1 (Figure 32 to Figure 34). Thus, a transient and ribosomemediated contact would be possible, as soon as Dbp5 and Gle1 enter the stop codon-containing ribosome.

\subsubsection{New translation termination model}

Thus, according to these results the following preliminary model for the chronology of all termination factors acting during translation termination could be suggested (Figure 40). 

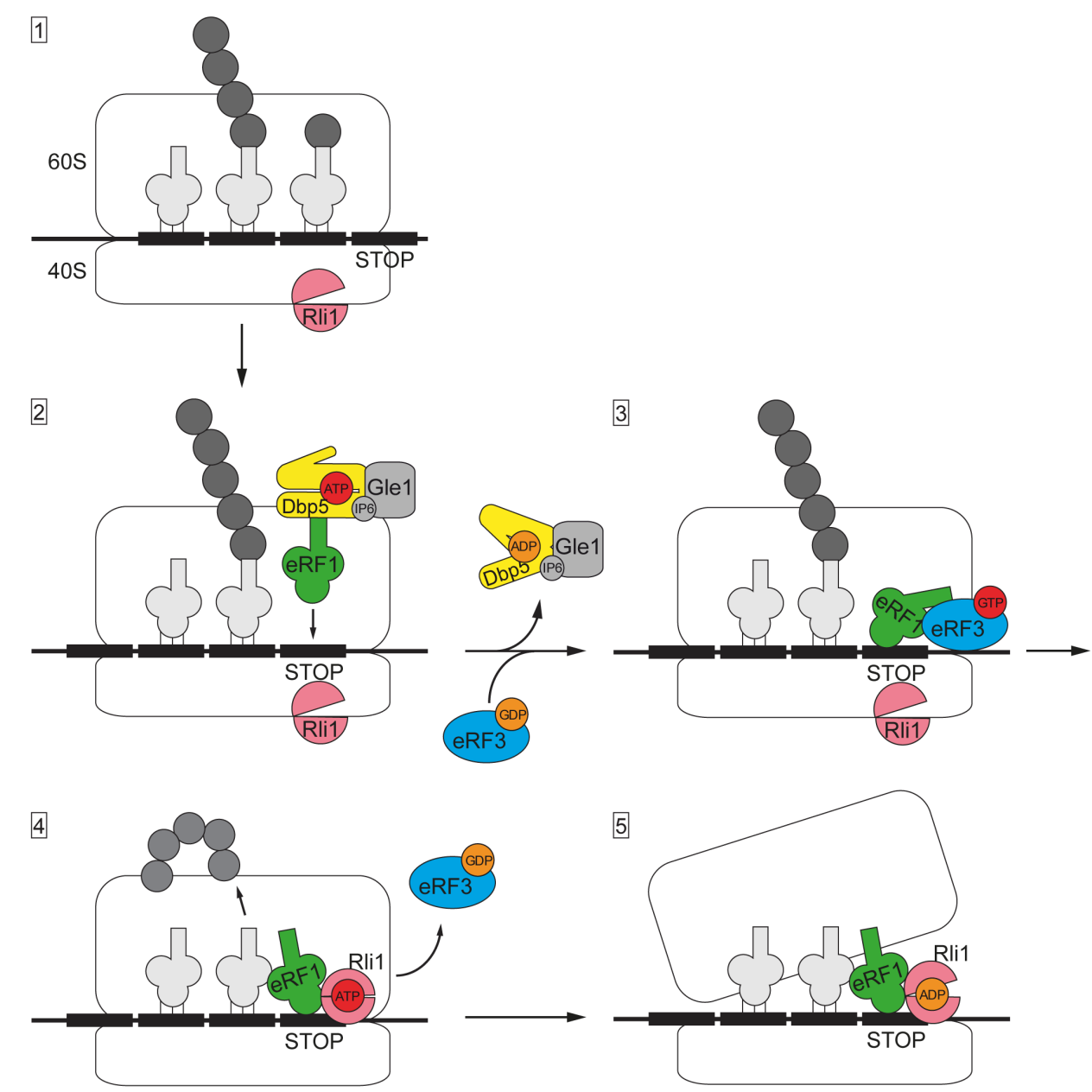

Figure 40: Suggested model for the chronology of translation termination.

Nucleotide-free Rli1 might already be loosely associated with the ribosomes during elongation (1). Upon arrival at the stop codon, eRF1, possibly together with Dbp5 and Gle1/IP ${ }_{6}$, is recruited and facilitates the stop codon recognition (2). Gle1/IP 6 dependent stimulation of the ATPase activity of Dbp5 and their subsequent dissociation might be needed for remodeling of the ribosomal complex and recruitment of eRF3-GDP (3). Binding to eRF1 might mediate GTP-binding by eRF3 and its ribosome and eRF1-dependent GTP-hydrolysis leads to proper positioning of eRF1 in the peptidyltransferase center to facilitate peptide release (3-4). Upon eRF3-GDP dissociation, ATP-bound Rli1 takes over the position of eRF3 and stabilizes eRF1 for efficient release of the polypeptide chain (4). Subsequently, Rli1 mediates the ATPase dependent splitting of the ribosomal subunits (5).

After its function in translation initiation (Chen et al., 2006; Dong et al., 2004), Rli1 might remain loosely associated with the $40 \mathrm{~S}$ subunit during translation elongation in a nucleotide-free conformation (Figure 40, step 1). Upon arrival at the stop codon, eRF1, possibly bound to Dbp5 and its co-factors Gle1 and $\mathrm{IP}_{6}$, enters the ribosomal A-site and facilitates the stop codon recognition (step 2). This process and the recruitment of eRF3 might be mediated by the Gle1 and $\mathrm{IP}_{6}$ stimulated ATPase activity of Dbp5, which might result in a remodeling of the ribosomal complex (Bolger et al., 2008; Gross et al., 2007). Gle1 and Dbp5 have to leave the terminating ribosome to enable the binding of eRF3 (step 3). As eRF3 alone has a higher affinity to GDP and binding to eRF1 increases the affinity for GTP (Pisareva et al., 2006), eRF3 should arrive in its GDP-bound form and the GTP-exchange might happen 
upon eRF1 contact at the ribosome. In its eRF3-GTP bound conformation, the GGQ motif of eRF1 has a large distance to the peptidyl-transferase center that is incompatible to mediate the peptide release (des Georges et al., 2014; Preis et al., 2014; Taylor et al., 2012). However, the ribosome- and eRF1-induced GTPhydrolysis of eRF3 causes a conformational rearrangement and the proper positioning of the GGQ motif into the peptidyl-transferase center facilitates the peptidyl-tRNA hydrolysis (step 4). Upon GTP-hydrolysis, eRF3-GDP dissociates from the ribosome and might allow the high affinity binding of ATP-bound Rli1 to the eRF3-free intersubunit space (Becker et al., 2012; Preis et al., 2014; Shoemaker and Green, 2011). Rli1 might stabilize the favorable conformation of eRF1, so that efficient peptide release is possible. Subsequent splitting of the ribosomal subunits is mediated by Rli1 and depends on its complete closure and ATP-hydrolysis (step 5) (Becker et al., 2012; Pisarev et al., 2010; Shoemaker and Green, 2011). Afterwards, Rli1 remains bound to the 40 s subunit.

Even though quite attractive, further studies are needed to fully uncover the process of translation termination. Especially the mechanism of how Dbp5 and Gle1 contribute to the termination process and cause a potential ATPase-dependent remodeling of the termination complex remains to be shown. Thus, crystal structure analyses should be performed with pre- and post-termination complexes that include Dbp5 and Gle1. Furthermore, it should be analyzed whether the ribosome entry of Dbp5 and Gle1 bound to free eRF1 is possible or whether a binding of eRF1-eRF3GTP in a ternary complex, as anticipated before, is favored. To analyze a potential mutually exclusive eRF1 binding of Dbp5 and eRF3, in vitro binding studies and competition assays with purified factors should be performed. Moreover, it might be possible that Rli1 also contributes to the stop codon recognition, as it is already bound to the ribosomes during the eRF1 recruitment. This possibility is corroborated by the fact that the stop codon read-through rate is increased in RLI1-depleted cells (Khoshnevis et al., 2010). However, this effect could also originate from the missing recycling of the release factors in these cells. Thus, further experiments are needed to support this possibility.

In conclusion, the present study suggests that translation termination involves an early ribosome-mediated contact between Rli1 and Dbp5, which might be enabled by the early recruitment of Rli1 to translating ribosomes prior their arrival at the stop codon. These novel findings are important to uncover the temporal coordination of all termination factors acting during translation termination. 


\section{REFERENCES}

Adams, R.L., Terry, L.J., and Wente, S.R. (2014). Nucleoporin FG domains facilitate mRNP remodeling at the cytoplasmic face of the nuclear pore complex. Genetics 197, 1213-1224.

Aibara, S., Valkov, E., Lamers, M., and Stewart, M. (2015). Domain organization within the nuclear export factor Mex67:Mtr2 generates an extended mRNA binding surface. Nucleic Acids Res 43, 1927-1936.

Alcazar-Roman, A.R., Bolger, T.A., and Wente, S.R. (2010). Control of mRNA export and translation termination by inositol hexakisphosphate requires specific interaction with Gle1. J Biol Chem 285, 16683-16692.

Alcazar-Roman, A.R., Tran, E.J., Guo, S., and Wente, S.R. (2006). Inositol hexakisphosphate and Gle1 activate the DEAD-box protein Dbp5 for nuclear mRNA export. Nat Cell Biol 8, 711-716.

Alkalaeva, E.Z., Pisarev, A.V., Frolova, L.Y., Kisselev, L.L., and Pestova, T.V. (2006). In vitro reconstitution of eukaryotic translation reveals cooperativity between release factors eRF1 and eRF3. Cell 125, 1125-1136.

Altvater, M., Chang, Y., Melnik, A., Occhipinti, L., Schutz, S., Rothenbusch, U., Picotti, P., and Panse, V.G. (2012). Targeted proteomics reveals compositional dynamics of 605 pre-ribosomes after nuclear export. Mol Syst Biol 8, 628.

Amberg, D.C., Goldstein, A.L., and Cole, C.N. (1992). Isolation and characterization of RAT1: an essential gene of Saccharomyces cerevisiae required for the efficient nucleocytoplasmic trafficking of mRNA. Genes Dev 6, 1173-1189.

Baierlein, C., Hackmann, A., Gross, T., Henker, L., Hinz, F., and Krebber, H. (2013). Monosome formation during translation initiation requires the serine/argininerich protein Npl3. Mol Cell Biol 33, 4811-4823.

Baierlein, C., and Krebber, H. (2010). Translation termination: new factors and insights. RNA Biol 7, 548-550.

Barthelme, D., Dinkelaker, S., Albers, S.V., Londei, P., Ermler, U., and Tampe, R. (2011). Ribosome recycling depends on a mechanistic link between the FeS cluster domain and a conformational switch of the twin-ATPase ABCE1. Proc Natl Acad Sci U S A 108, 3228-3233.

Barthelme, D., Scheele, U., Dinkelaker, S., Janoschka, A., Macmillan, F., Albers, S.V., Driessen, A.J., Stagni, M.S., Bill, E., Meyer-Klaucke, W., et al. (2007). Structural organization of essential iron-sulfur clusters in the evolutionarily highly conserved ATP-binding cassette protein ABCE1. J Biol Chem 282, 14598-14607.

Bassler, J., Grandi, P., Gadal, O., Lessmann, T., Petfalski, E., Tollervey, D., Lechner, J., and Hurt, E. (2001). Identification of a 605 preribosomal particle that is closely linked to nuclear export. Mol Cell 8, 517-529.

Bassler, J., Klein, I., Schmidt, C., Kallas, M., Thomson, E., Wagner, M.A., Bradatsch, B., Rechberger, G., Strohmaier, H., Hurt, E., et al. (2012). The conserved Bud20 zinc finger protein is a new component of the ribosomal 605 subunit export machinery. Mol Cell Biol 32, 4898-4912.

Becker, T., Franckenberg, S., Wickles, S., Shoemaker, C.J., Anger, A.M., Armache, J.P., Sieber, H., Ungewickell, C., Berninghausen, O., Daberkow, I., et al. (2012). Structural basis of highly conserved ribosome recycling in eukaryotes and archaea. Nature 482, 501-506.

Beznoskova, P., Cuchalova, L., Wagner, S., Shoemaker, C.J., Gunisova, S., von der Haar, T., and Valasek, L.S. (2013). Translation initiation factors elF3 and HCR1 
control translation termination and stop codon read-through in yeast cells. PLoS Genet 9, e1003962.

Bisbal, C., Martinand, C., Silhol, M., Lebleu, B., and Salehzada, T. (1995). Cloning and characterization of a RNAse $L$ inhibitor. A new component of the interferonregulated 2-5A pathway. J Biol Chem 270, 13308-13317.

Block, H., Maertens, B., Spriestersbach, A., Brinker, N., Kubicek, J., Fabis, R., Labahn, J., and Schafer, F. (2009). Immobilized-metal affinity chromatography (IMAC): a review. Methods Enzymol 463, 439-473.

Bolger, T.A., Folkmann, A.W., Tran, E.J., and Wente, S.R. (2008). The mRNA export factor Gle1 and inositol hexakisphosphate regulate distinct stages of translation. Cell 134, 624-633.

Bolger, T.A., and Wente, S.R. (2011). Gle1 is a multifunctional DEAD-box protein regulator that modulates Ded1 in translation initiation. J Biol Chem 286, 3975039759.

Bradatsch, B., Katahira, J., Kowalinski, E., Bange, G., Yao, W., Sekimoto, T., Baumgartel, V., Boese, G., Bassler, J., Wild, K., et al. (2007). Arx1 functions as an unorthodox nuclear export receptor for the $60 \mathrm{~S}$ preribosomal subunit. Mol Cell 27, 767-779.

Chen, J., Lu, G., Lin, J., Davidson, A.L., and Quiocho, F.A. (2003). A tweezers-like motion of the ATP-binding cassette dimer in an ABC transport cycle. Mol Cell $12,651-661$.

Chen, Z.Q., Dong, J., Ishimura, A., Daar, I., Hinnebusch, A.G., and Dean, M. (2006). The essential vertebrate $A B C E 1$ protein interacts with eukaryotic initiation factors. J Biol Chem 281, 7452-7457.

Cheng, Z., Saito, K., Pisarev, A.V., Wada, M., Pisareva, V.P., Pestova, T.V., Gajda, M., Round, A., Kong, C., Lim, M., et al. (2009). Structural insights into eRF3 and stop codon recognition by eRF1. Genes Dev 23, 1106-1118.

Ciganda, M., and Williams, N. (2011). Eukaryotic 5S rRNA biogenesis. Wiley Interdiscip Rev RNA 2, 523-533.

Collins, R., Karlberg, T., Lehtio, L., Schutz, P., van den Berg, S., Dahlgren, L.G., Hammarstrom, M., Weigelt, J., and Schuler, H. (2009). The DEXD/H-box RNA helicase DDX19 is regulated by an \{alpha\}-helical switch. J Biol Chem 284, 10296-10300.

Del Priore, V., Heath, C., Snay, C., MacMillan, A., Gorsch, L., Dagher, S., and Cole, C. (1997). A structure/function analysis of Rat7p/Nup159p, an essential nucleoporin of Saccharomyces cerevisiae. J Cell Sci 110 ( Pt 23), 2987-2999.

Del Priore, V., Snay, C.A., Bahr, A., and Cole, C.N. (1996). The product of the Saccharomyces cerevisiae RSS1 gene, identified as a high-copy suppressor of the rat7-1 temperature-sensitive allele of the RAT7/NUP159 nucleoporin, is required for efficient mRNA export. Mol Biol Cell 7, 1601-1621.

des Georges, A., Hashem, Y., Unbehaun, A., Grassucci, R.A., Taylor, D., Hellen, C.U., Pestova, T.V., and Frank, J. (2014). Structure of the mammalian ribosomal pre-termination complex associated with eRF1.eRF3.GDPNP. Nucleic Acids Res 42, 3409-3418.

Dever, T.E., and Green, R. (2012). The elongation, termination, and recycling phases of translation in eukaryotes. Cold Spring Harb Perspect Biol 4, a013706.

Dong, J., Lai, R., Nielsen, K., Fekete, C.A., Qiu, H., and Hinnebusch, A.G. (2004). The essential ATP-binding cassette protein RLI1 functions in translation by promoting preinitiation complex assembly. J Biol Chem 279, 42157-42168.

Dossani, Z.Y., Weirich, C.S., Erzberger, J.P., Berger, J.M., and Weis, K. (2009). Structure of the C-terminus of the mRNA export factor Dbp5 reveals the interaction surface for the ATPase activator Gle1. Proc Natl Acad Sci U S A 
$106,16251-16256$.

Ebihara, K., and Nakamura, Y. (1999). C-terminal interaction of translational release factors eRF1 and eRF3 of fission yeast: G-domain uncoupled binding and the role of conserved amino acids. RNA 5, 739-750.

Estruch, F., and Cole, C.N. (2003). An early function during transcription for the yeast mRNA export factor Dbp5p/Rat8p suggested by its genetic and physical interactions with transcription factor IIH components. Mol Biol Cell 14, 16641676.

Estruch, F., Hodge, C., Gomez-Navarro, N., Peiro-Chova, L., Heath, C.V., and Cole, C.N. (2012). Insights into mRNP biogenesis provided by new genetic interactions among export and transcription factors. BMC Genet 13, 80.

Eurwilaichitr, L., Graves, F.M., Stansfield, I., and Tuite, M.F. (1999). The C-terminus of eRF1 defines a functionally important domain for translation termination in Saccharomyces cerevisiae. Mol Microbiol 32, 485-496.

Eyler, D.E., Wehner, K.A., and Green, R. (2013). Eukaryotic release factor 3 is required for multiple turnovers of peptide release catalysis by eukaryotic release factor 1. J Biol Chem 288, 29530-29538.

Fan, J.S., Cheng, Z., Zhang, J., Noble, C., Zhou, Z., Song, H., and Yang, D. (2009). Solution and crystal structures of mRNA exporter Dbp5p and its interaction with nucleotides. J Mol Biol 388, 1-10.

Faza, M.B., Chang, Y., Occhipinti, L., Kemmler, S., and Panse, V.G. (2012). Role of Mex67-Mtr2 in the nuclear export of $40 \mathrm{~S}$ pre-ribosomes. PLoS Genet 8 , e1002915.

Folkmann, A.W., Collier, S.E., Zhan, X., Aditi, Ohi, M.D., and Wente, S.R. (2013). Gle1 functions during mRNA export in an oligomeric complex that is altered in human disease. Cell 155, 582-593.

Folkmann, A.W., Noble, K.N., Cole, C.N., and Wente, S.R. (2011). Dbp5, Gle1-IP6 and Nup159: a working model for mRNP export. Nucleus 2, 540-548.

Fornerod, M., Ohno, M., Yoshida, M., and Mattaj, I.W. (1997). CRM1 is an export receptor for leucine-rich nuclear export signals. Cell 90, 1051-1060.

Franckenberg, S., Becker, T., and Beckmann, R. (2012). Structural view on recycling of archaeal and eukaryotic ribosomes after canonical termination and ribosome rescue. Curr Opin Struct Biol 22, 786-796.

Frey, S., Pool, M., and Seedorf, M. (2001). Scp160p, an RNA-binding, polysomeassociated protein, localizes to the endoplasmic reticulum of Saccharomyces cerevisiae in a microtubule-dependent manner. J Biol Chem 276, 15905-15912.

Frolova, L., Le Goff, X., Rasmussen, H.H., Cheperegin, S., Drugeon, G., Kress, M., Arman, I., Haenni, A.L., Celis, J.E., Philippe, M., et al. (1994). A highly conserved eukaryotic protein family possessing properties of polypeptide chain release factor. Nature $372,701-703$.

Frolova, L., Le Goff, X., Zhouravleva, G., Davydova, E., Philippe, M., and Kisselev, L. (1996). Eukaryotic polypeptide chain release factor eRF3 is an eRF1- and ribosome-dependent guanosine triphosphatase. RNA 2, 334-341.

Gadal, O., Strauss, D., Kessl, J., Trumpower, B., Tollervey, D., and Hurt, E. (2001). Nuclear export of 60s ribosomal subunits depends on Xpo1p and requires a nuclear export sequence-containing factor, Nmd3p, that associates with the large subunit protein Rpl10p. Mol Cell Biol 21, 3405-3415.

Gallagher, J.E., Dunbar, D.A., Granneman, S., Mitchell, B.M., Osheim, Y., Beyer, A.L., and Baserga, S.J. (2004). RNA polymerase I transcription and pre-rRNA processing are linked by specific SSU processome components. Genes Dev 18, 2506-2517. 
Garfin, D.E. (2009). One-dimensional gel electrophoresis. Methods Enzymol 463, 497-513.

Garre, E., Romero-Santacreu, L., De Clercq, N., Blasco-Angulo, N., Sunnerhagen, P., and Alepuz, P. (2012). Yeast mRNA cap-binding protein Cbc1/Sto1 is necessary for the rapid reprogramming of translation after hyperosmotic shock. Mol Biol Cell 23, 137-150.

Gerhardy, S., Menet, A.M., Pena, C., Petkowski, J.J., and Panse, V.G. (2014). Assembly and nuclear export of pre-ribosomal particles in budding yeast. Chromosoma.

Gibson, D.G. (2011). Enzymatic assembly of overlapping DNA fragments. Methods Enzymol 498, 349-361.

Gibson, D.G., Young, L., Chuang, R.Y., Venter, J.C., Hutchison, C.A., 3rd, and Smith, H.O. (2009). Enzymatic assembly of DNA molecules up to several hundred kilobases. Nat Methods 6, 343-345.

Gietz, D., St Jean, A., Woods, R.A., and Schiestl, R.H. (1992). Improved method for high efficiency transformation of intact yeast cells. Nucleic Acids Res 20, 1425.

Gleizes, P.E., Noaillac-Depeyre, J., Leger-Silvestre, I., Teulieres, F., Dauxois, J.Y., Pommet, D., Azum-Gelade, M.C., and Gas, N. (2001). Ultrastructural localization of rRNA shows defective nuclear export of preribosomes in mutants of the Nup82p complex. J Cell Biol 155, 923-936.

Gorsch, L.C., Dockendorff, T.C., and Cole, C.N. (1995). A conditional allele of the novel repeat-containing yeast nucleoporin RAT7/NUP159 causes both rapid cessation of mRNA export and reversible clustering of nuclear pore complexes. J Cell Biol 129, 939-955.

Gross, T., Siepmann, A., Sturm, D., Windgassen, M., Scarcelli, J.J., Seedorf, M., Cole, C.N., and Krebber, H. (2007). The DEAD-box RNA helicase Dbp5 functions in translation termination. Science 315, 646-649.

Grunwald, D., Singer, R.H., and Rout, M. (2011). Nuclear export dynamics of RNAprotein complexes. Nature 475, 333-341.

Hackmann, A., Gross, T., Baierlein, C., and Krebber, H. (2011). The mRNA export factor Npl3 mediates the nuclear export of large ribosomal subunits. EMBO Rep 12, 1024-1031.

Hackmann, A., Wu, H., Schneider, U.M., Meyer, K., Jung, K., and Krebber, H. (2014). Quality control of spliced mRNAs requires the shuttling SR proteins Gbp2 and Hrb1. Nat Commun 5, 3123.

Harper, S., and Speicher, D.W. (2011). Purification of proteins fused to glutathione Stransferase. Methods Mol Biol 681, 259-280.

Hedges, J., West, M., and Johnson, A.W. (2005). Release of the export adapter, Nmd3p, from the 60S ribosomal subunit requires Rpl10p and the cytoplasmic GTPase Lsg1p. EMBO J 24, 567-579.

Ho, J.H., and Johnson, A.W. (1999). NMD3 encodes an essential cytoplasmic protein required for stable $60 \mathrm{~S}$ ribosomal subunits in Saccharomyces cerevisiae. Mol Cell Biol 19, 2389-2399.

Ho, J.H., Kallstrom, G., and Johnson, A.W. (2000). Nmd3p is a Crm1p-dependent adapter protein for nuclear export of the large ribosomal subunit. J Cell Biol 151, 1057-1066.

Hodge, C.A., Colot, H.V., Stafford, P., and Cole, C.N. (1999). Rat8p/Dbp5p is a shuttling transport factor that interacts with Rat7p/Nup159p and Gle1p and suppresses the mRNA export defect of xpo1-1 cells. EMBO J 18, 5778-5788.

Hodge, C.A., Tran, E.J., Noble, K.N., Alcazar-Roman, A.R., Ben-Yishay, R., Scarcelli, J.J., Folkmann, A.W., Shav-Tal, Y., Wente, S.R., and Cole, C.N. (2011). The Dbp5 cycle at the nuclear pore complex during mRNA export I: dbp5 mutants 
with defects in RNA binding and ATP hydrolysis define key steps for Nup159 and Gle1. Genes Dev 25, 1052-1064.

Hoshino, S., Imai, M., Kobayashi, T., Uchida, N., and Katada, T. (1999). The eukaryotic polypeptide chain releasing factor (eRF3/GSPT) carrying the translation termination signal to the 3'-Poly(A) tail of mRNA. Direct association of erf3/GSPT with polyadenylate-binding protein. J Biol Chem 274, 1667716680.

Hoshino, S., Imai, M., Mizutani, M., Kikuchi, Y., Hanaoka, F., Ui, M., and Katada, T. (1998). Molecular cloning of a novel member of the eukaryotic polypeptide chain-releasing factors (eRF). Its identification as eRF3 interacting with eRF1. J Biol Chem 273, 22254-22259.

Hung, N.J., Lo, K.Y., Patel, S.S., Helmke, K., and Johnson, A.W. (2008). Arx1 is a nuclear export receptor for the $60 \mathrm{~S}$ ribosomal subunit in yeast. Mol Biol Cell 19, 735-744.

Hurt, E., Hannus, S., Schmelzl, B., Lau, D., Tollervey, D., and Simos, G. (1999). A novel in vivo assay reveals inhibition of ribosomal nuclear export in ran-cycle and nucleoporin mutants. J Cell Biol 144, 389-401.

Inge-Vechtomov, S., Zhouravleva, G., and Philippe, M. (2003). Eukaryotic release factors (eRFs) history. Biol Cell 95, 195-209.

Inoue, H., Nojima, H., and Okayama, H. (1990). High efficiency transformation of Escherichia coli with plasmids. Gene 96, 23-28.

Ito, K., Ebihara, K., and Nakamura, Y. (1998). The stretch of C-terminal acidic amino acids of translational release factor eRF1 is a primary binding site for eRF3 of fission yeast. RNA 4, 958-972.

Ito, K., Ebihara, K., Uno, M., and Nakamura, Y. (1996). Conserved motifs in prokaryotic and eukaryotic polypeptide release factors: tRNA-protein mimicry hypothesis. Proc Natl Acad Sci U S A 93, 5443-5448.

Jackson, R.J., Hellen, C.U., and Pestova, T.V. (2012). Termination and posttermination events in eukaryotic translation. Adv Protein Chem Struct Biol 86, 45-93.

Kappel, L., Loibl, M., Zisser, G., Klein, I., Fruhmann, G., Gruber, C., Unterweger, S., Rechberger, G., Pertschy, B., and Bergler, H. (2012). RIp24 activates the AAAATPase Drg1 to initiate cytoplasmic pre-60S maturation. J Cell Biol 199, 771782.

Karbstein, K. (2013). Quality control mechanisms during ribosome maturation. Trends Cell Biol 23, 242-250.

Karcher, A., Buttner, K., Martens, B., Jansen, R.P., and Hopfner, K.P. (2005). X-ray structure of $\mathrm{RLI}$, an essential twin cassette $A B C$ ATPase involved in ribosome biogenesis and HIV capsid assembly. Structure 13, 649-659.

Karcher, A., Schele, A., and Hopfner, K.P. (2008). X-ray structure of the complete ABC enzyme ABCE1 from Pyrococcus abyssi. J Biol Chem 283, 7962-7971.

Kelly, S.M., and Corbett, A.H. (2009). Messenger RNA export from the nucleus: a series of molecular wardrobe changes. Traffic 10, 1199-1208.

Khoshnevis, S., Gross, T., Rotte, C., Baierlein, C., Ficner, R., and Krebber, H. (2010). The iron-sulphur protein RNase $\mathrm{L}$ inhibitor functions in translation termination. EMBO Rep 11, 214-219.

Kispal, G., Sipos, K., Lange, H., Fekete, Z., Bedekovics, T., Janaky, T., Bassler, J., Aguilar Netz, D.J., Balk, J., Rotte, C., et al. (2005). Biogenesis of cytosolic ribosomes requires the essential iron-sulphur protein Rli1p and mitochondria. EMBO J 24, 589-598.

Kong, C., Ito, K., Walsh, M.A., Wada, M., Liu, Y., Kumar, S., Barford, D., Nakamura, Y., and Song, H. (2004). Crystal structure and functional analysis of the 
eukaryotic class II release factor eRF3 from S. pombe. Mol Cell 14, 233-245.

Kononenko, A.V., Mitkevich, V.A., Dubovaya, V.I., Kolosov, P.M., Makarov, A.A., and Kisselev, L.L. (2008). Role of the individual domains of translation termination factor eRF1 in GTP binding to eRF3. Proteins 70, 388-393.

Kressler, D., Hurt, E., and Bassler, J. (2010). Driving ribosome assembly. Biochim Biophys Acta 1803, 673-683.

Kryuchkova, P., Grishin, A., Eliseev, B., Karyagina, A., Frolova, L., and Alkalaeva, E. (2013). Two-step model of stop codon recognition by eukaryotic release factor eRF1. Nucleic Acids Res 41, 4573-4586.

Laemmli, U.K. (1970). Cleavage of structural proteins during the assembly of the head of bacteriophage T4. Nature 227, 680-685.

Li, Z., Lee, I., Moradi, E., Hung, N.J., Johnson, A.W., and Marcotte, E.M. (2009). Rational extension of the ribosome biogenesis pathway using network-guided genetics. PLoS Biol 7, e1000213.

Linder, P., and Jankowsky, E. (2011). From unwinding to clamping - the DEAD box RNA helicase family. Nat Rev Mol Cell Biol 12, 505-516.

Lo, K.Y., Li, Z., Bussiere, C., Bresson, S., Marcotte, E.M., and Johnson, A.W. (2010). Defining the pathway of cytoplasmic maturation of the $60 \mathrm{~S}$ ribosomal subunit. Mol Cell 39, 196-208.

Lund, M.K., and Guthrie, C. (2005). The DEAD-box protein Dbp5p is required to dissociate Mex67p from exported mRNPs at the nuclear rim. Mol Cell 20,645651.

Masek, T., Valasek, L., and Pospisek, M. (2011). Polysome analysis and RNA purification from sucrose gradients. Methods Mol Biol 703, 293-309.

Matsuo, Y., Granneman, S., Thoms, M., Manikas, R.G., Tollervey, D., and Hurt, E. (2014). Coupled GTPase and remodelling ATPase activities form a checkpoint for ribosome export. Nature 505, 112-116.

Merkulova, T.I., Frolova, L.Y., Lazar, M., Camonis, J., and Kisselev, L.L. (1999). Cterminal domains of human translation termination factors eRF1 and eRF3 mediate their in vivo interaction. FEBS Lett 443, 41-47.

Merwin, J.R., Bogar, L.B., Poggi, S.B., Fitch, R.M., Johnson, A.W., and Lycan, D.E. (2014). Genetic Analysis of the Ribosome Biogenesis Factor Ltv1 of Saccharomyces cerevisiae. Genetics 198, 1071-1085.

Milkereit, P., Strauss, D., Bassler, J., Gadal, O., Kuhn, H., Schutz, S., Gas, N., Lechner, J., Hurt, E., and Tschochner, H. (2003). A Noc complex specifically involved in the formation and nuclear export of ribosomal $40 \mathrm{~S}$ subunits. J Biol Chem 278, 4072-4081.

Montpetit, B., Thomsen, N.D., Helmke, K.J., Seeliger, M.A., Berger, J.M., and Weis, K. (2011). A conserved mechanism of DEAD-box ATPase activation by nucleoporins and InsP6 in mRNA export. Nature 472, 238-242.

Moriggi, G., Nieto, B., and Dosil, M. (2014). Rrp12 and the Exportin Crm1 Participate in Late Assembly Events in the Nucleolus during 40S Ribosomal Subunit Biogenesis. PLoS Genet 10, e1004836.

Moy, T.I., and Silver, P.A. (1999). Nuclear export of the small ribosomal subunit requires the ran-GTPase cycle and certain nucleoporins. Genes Dev 13, 21182133.

Moy, T.I., and Silver, P.A. (2002). Requirements for the nuclear export of the small ribosomal subunit. J Cell Sci 115, 2985-2995.

Murphy, R., and Wente, S.R. (1996). An RNA-export mediator with an essential nuclear export signal. Nature 383, 357-360.

Napetschnig, J., Kassube, S.A., Debler, E.W., Wong, R.W., Blobel, G., and Hoelz, A. (2009). Structural and functional analysis of the interaction between the 
nucleoporin Nup214 and the DEAD-box helicase Ddx19. Proc Natl Acad Sci U S A 106, 3089-3094.

Noble, K.N., Tran, E.J., Alcazar-Roman, A.R., Hodge, C.A., Cole, C.N., and Wente, S.R. (2011). The Dbp5 cycle at the nuclear pore complex during mRNA export II: nucleotide cycling and mRNP remodeling by Dbp5 are controlled by Nup159 and Gle1. Genes Dev 25, 1065-1077.

Nousiainen, H.O., Kestila, M., Pakkasjarvi, N., Honkala, H., Kuure, S., Tallila, J., Vuopala, K., Ignatius, J., Herva, R., and Peltonen, L. (2008). Mutations in mRNA export mediator GLE1 result in a fetal motoneuron disease. Nat Genet 40, 155-157.

Nurenberg, E., and Tampe, R. (2013). Tying up loose ends: ribosome recycling in eukaryotes and archaea. Trends Biochem Sci 38, 64-74.

Oeffinger, M., Dlakic, M., and Tollervey, D. (2004). A pre-ribosome-associated HEATrepeat protein is required for export of both ribosomal subunits. Genes Dev 18, 196-209.

Panse, V.G., and Johnson, A.W. (2010). Maturation of eukaryotic ribosomes: acquisition of functionality. Trends Biochem Sci 35, 260-266.

Paushkin, S.V., Kushnirov, V.V., Smirnov, V.N., and Ter-Avanesyan, M.D. (1997). Interaction between yeast Sup45p (eRF1) and Sup35p (eRF3) polypeptide chain release factors: implications for prion-dependent regulation. Mol Cell Biol 17, 2798-2805.

Perez-Ortin, J.E., Medina, D.A., and Jordan-Pla, A. (2011). Genomic insights into the different layers of gene regulation in yeast. Genet Res Int 2011, 989303.

Pertschy, B., Saveanu, C., Zisser, G., Lebreton, A., Tengg, M., Jacquier, A., Liebminger, E., Nobis, B., Kappel, L., van der Klei, I., et al. (2007). Cytoplasmic recycling of 605 preribosomal factors depends on the AAA protein Drg1. Mol Cell Biol 27, 6581-6592.

Pisarev, A.V., Hellen, C.U., and Pestova, T.V. (2007). Recycling of eukaryotic posttermination ribosomal complexes. Cell 131, 286-299.

Pisarev, A.V., Skabkin, M.A., Pisareva, V.P., Skabkina, O.V., Rakotondrafara, A.M., Hentze, M.W., Hellen, C.U., and Pestova, T.V. (2010). The role of ABCE1 in eukaryotic posttermination ribosomal recycling. Mol Cell 37, 196-210.

Pisareva, V.P., Pisarev, A.V., Hellen, C.U., Rodnina, M.V., and Pestova, T.V. (2006). Kinetic analysis of interaction of eukaryotic release factor 3 with guanine nucleotides. J Biol Chem 281, 40224-40235.

Pisareva, V.P., Skabkin, M.A., Hellen, C.U., Pestova, T.V., and Pisarev, A.V. (2011). Dissociation by Pelota, Hbs1 and ABCE1 of mammalian vacant $80 \mathrm{~S}$ ribosomes and stalled elongation complexes. EMBO J 30, 1804-1817.

Preis, A., Heuer, A., Barrio-Garcia, C., Hauser, A., Eyler, D.E., Berninghausen, O., Green, R., Becker, T., and Beckmann, R. (2014). Cryoelectron microscopic structures of eukaryotic translation termination complexes containing eRF1eRF3 or eRF1-ABCE1. Cell Rep 8, 59-65.

Rigaut, G., Shevchenko, A., Rutz, B., Wilm, M., Mann, M., and Seraphin, B. (1999). A generic protein purification method for protein complex characterization and proteome exploration. Nat Biotechnol 17, 1030-1032.

Roque, S., Cerciat, M., Gaugue, I., Mora, L., Floch, A.G., de Zamaroczy, M., Heurgue-Hamard, V., and Kervestin, S. (2014). Interaction between the poly(A)binding protein $\mathrm{Pab1}$ and the eukaryotic release factor eRF3 regulates translation termination but not mRNA decay in Saccharomyces cerevisiae. RNA.

Rose, M.D., Winston, F., and Hieter, P. (1990). Methods in Yeast Genetics, A Laboratory Course Manual. (New York: Cold Spring Harbor Laboratory Press). 
Salas-Marco, J., and Bedwell, D.M. (2004). GTP hydrolysis by eRF3 facilitates stop codon decoding during eukaryotic translation termination. Mol Cell Biol 24, 7769-7778.

Sambrook, J., Fritsch, E.F., and Maniatis, T. (1989). Molecular Cloning: a laboratory manual. (New York: Cold Spring Harbor Laboratory Press).

Santos-Rosa, H., Moreno, H., Simos, G., Segref, A., Fahrenkrog, B., Pante, N., and Hurt, E. (1998). Nuclear mRNA export requires complex formation between Mex67p and Mtr2p at the nuclear pores. Mol Cell Biol 18, 6826-6838.

Scarcelli, J.J., Viggiano, S., Hodge, C.A., Heath, C.V., Amberg, D.C., and Cole, C.N. (2008). Synthetic genetic array analysis in Saccharomyces cerevisiae provides evidence for an interaction between RAT8/DBP5 and genes encoding P-body components. Genetics 179, 1945-1955.

Schafer, T., Maco, B., Petfalski, E., Tollervey, D., Bottcher, B., Aebi, U., and Hurt, E. (2006). Hrr25-dependent phosphorylation state regulates organization of the pre-40S subunit. Nature 441, 651-655.

Schafer, T., Strauss, D., Petfalski, E., Tollervey, D., and Hurt, E. (2003). The path from nucleolar $90 \mathrm{~S}$ to cytoplasmic $40 \mathrm{~S}$ pre-ribosomes. EMBO J 22, 1370-1380.

Schmitt, C., von Kobbe, C., Bachi, A., Pante, N., Rodrigues, J.P., Boscheron, C., Rigaut, G., Wilm, M., Seraphin, B., Carmo-Fonseca, M., et al. (1999). Dbp5, a DEAD-box protein required for mRNA export, is recruited to the cytoplasmic fibrils of nuclear pore complex via a conserved interaction with CAN/Nup159p. EMBO J 18, 4332-4347.

Segref, A., Sharma, K., Doye, V., Hellwig, A., Huber, J., Luhrmann, R., and Hurt, E. (1997). Mex67p, a novel factor for nuclear mRNA export, binds to both poly(A)+ RNA and nuclear pores. EMBO J 16, 3256-3271.

Seiser, R.M., Sundberg, A.E., Wollam, B.J., Zobel-Thropp, P., Baldwin, K., Spector, M.D., and Lycan, D.E. (2006). Ltv1 is required for efficient nuclear export of the ribosomal small subunit in Saccharomyces cerevisiae. Genetics 174, 679-691.

Shen, E.C., Stage-Zimmermann, T., Chui, P., and Silver, P.A. (2000). 7The yeast mRNA-binding protein Npl3p interacts with the cap-binding complex. J Biol Chem 275, 23718-23724.

Sherman, F. (1991). Getting started with yeast. Methods Enzymol 194, 3-21.

Sherman, F., and Hicks, J. (1991). Micromanipulation and dissection of asci. Methods Enzymol 194, 21-37.

Shoemaker, C.J., and Green, R. (2011). Kinetic analysis reveals the ordered coupling of translation termination and ribosome recycling in yeast. Proc Natl Acad Sci U S A 108, E1392-1398.

Siebrasse, J.P., Kaminski, T., and Kubitscheck, U. (2012). Nuclear export of single native mRNA molecules observed by light sheet fluorescence microscopy. Proc Natl Acad Sci U S A 109, 9426-9431.

Sikorski, R.S., and Hieter, P. (1989). A system of shuttle vectors and yeast host strains designed for efficient manipulation of DNA in Saccharomyces cerevisiae. Genetics 122, 19-27.

Snay-Hodge, C.A., Colot, H.V., Goldstein, A.L., and Cole, C.N. (1998). Dbp5p/Rat8p is a yeast nuclear pore-associated DEAD-box protein essential for RNA export. EMBO J 17, 2663-2676.

Song, H., Mugnier, P., Das, A.K., Webb, H.M., Evans, D.R., Tuite, M.F., Hemmings, B.A., and Barford, D. (2000). The crystal structure of human eukaryotic release factor eRF1--mechanism of stop codon recognition and peptidyl-tRNA hydrolysis. Cell 100, 311-321.

Sprague, G.F., Jr. (1991). Assay of yeast mating reaction. Methods Enzymol 194, 7793. 
Stade, K., Ford, C.S., Guthrie, C., and Weis, K. (1997). Exportin 1 (Crm1p) is an essential nuclear export factor. Cell 90, 1041-1050.

Stage-Zimmermann, T., Schmidt, U., and Silver, P.A. (2000). Factors affecting nuclear export of the 605 ribosomal subunit in vivo. Mol Biol Cell 11, 37773789.

Stansfield, I., Grant, G.M., Akhmaloka, and Tuite, M.F. (1992). Ribosomal association of the yeast SAL4 (SUP45) gene product: implications for its role in translation fidelity and termination. Mol Microbiol 6, 3469-3478.

Stansfield, I., Jones, K.M., Kushnirov, V.V., Dagkesamanskaya, A.R., Poznyakovski, A.I., Paushkin, S.V., Nierras, C.R., Cox, B.S., Ter-Avanesyan, M.D., and Tuite, M.F. (1995). The products of the SUP45 (eRF1) and SUP35 genes interact to mediate translation termination in Saccharomyces cerevisiae. EMBO J 14, 4365-4373.

Stansfield, I., Kushnirov, V.V., Jones, K.M., and Tuite, M.F. (1997). A conditionallethal translation termination defect in a sup45 mutant of the yeast Saccharomyces cerevisiae. Eur J Biochem 245, 557-563.

Stewart, M. (2010). Nuclear export of mRNA. Trends Biochem Sci 35, 609-617.

Strahm, Y., Fahrenkrog, B., Zenklusen, D., Rychner, E., Kantor, J., Rosbach, M., and Stutz, F. (1999). The RNA export factor Gle1p is located on the cytoplasmic fibrils of the NPC and physically interacts with the FG-nucleoporin Rip1p, the DEAD-box protein Rat8p/Dbp5p and a new protein Ymr 255p. EMBO J 18, 5761-5777.

Strambio-De-Castillia, C., Niepel, M., and Rout, M.P. (2010). The nuclear pore complex: bridging nuclear transport and gene regulation. Nat Rev Mol Cell Biol 11, 490-501.

Strasser, K., Bassler, J., and Hurt, E. (2000). Binding of the Mex67p/Mtr2p heterodimer to FXFG, GLFG, and FG repeat nucleoporins is essential for nuclear mRNA export. J Cell Biol 150, 695-706.

Strunk, B.S., Loucks, C.R., Su, M., Vashisth, H., Cheng, S., Schilling, J., Brooks, C.L., 3rd, Karbstein, K., and Skiniotis, G. (2011). Ribosome assembly factors prevent premature translation initiation by $40 \mathrm{~S}$ assembly intermediates. Science 333, 1449-1453.

Strunk, B.S., Novak, M.N., Young, C.L., and Karbstein, K. (2012). A translation-like cycle is a quality control checkpoint for maturing $40 \mathrm{~S}$ ribosome subunits. Cell $150,111-121$.

Taylor, D., Unbehaun, A., Li, W., Das, S., Lei, J., Liao, H.Y., Grassucci, R.A., Pestova, T.V., and Frank, J. (2012). Cryo-EM structure of the mammalian eukaryotic release factor eRF1-eRF3-associated termination complex. Proc Natl Acad Sci U S A 109, 18413-18418.

Ter-Avanesyan, M.D., Kushnirov, V.V., Dagkesamanskaya, A.R., Didichenko, S.A., Chernoff, Y.O., Inge-Vechtomov, S.G., and Smirnov, V.N. (1993). Deletion analysis of the SUP35 gene of the yeast Saccharomyces cerevisiae reveals two non-overlapping functional regions in the encoded protein. Mol Microbiol 7, 683692.

Thomas, F., and Kutay, U. (2003). Biogenesis and nuclear export of ribosomal subunits in higher eukaryotes depend on the CRM1 export pathway. J Cell Sci 116, 2409-2419.

Thomson, E., Ferreira-Cerca, S., and Hurt, E. (2013). Eukaryotic ribosome biogenesis at a glance. J Cell Sci 126, 4815-4821.

Tieg, B., and Krebber, H. (2013). Dbp5 - from nuclear export to translation. Biochim Biophys Acta 1829, 791-798. 
Towbin, H., Staehelin, T., and Gordon, J. (1979). Electrophoretic transfer of proteins from polyacrylamide gels to nitrocellulose sheets: procedure and some applications. Proc Natl Acad Sci U S A 76, 4350-4354.

Tran, E.J., Zhou, Y., Corbett, A.H., and Wente, S.R. (2007). The DEAD-box protein Dbp5 controls mRNA export by triggering specific RNA:protein remodeling events. Mol Cell 28, 850-859.

Trotta, C.R., Lund, E., Kahan, L., Johnson, A.W., and Dahlberg, J.E. (2003). Coordinated nuclear export of 60S ribosomal subunits and NMD3 in vertebrates. EMBO J 22, 2841-2851.

Tschochner, H., and Hurt, E. (2003). Pre-ribosomes on the road from the nucleolus to the cytoplasm. Trends Cell Biol 13, 255-263.

Tseng, S.S., Weaver, P.L., Liu, Y., Hitomi, M., Tartakoff, A.M., and Chang, T.H. (1998). Dbp5p, a cytosolic RNA helicase, is required for poly(A)+ RNA export. EMBO J 17, 2651-2662.

Tuck, A.C., and Tollervey, D. (2013). A transcriptome-wide atlas of RNP composition reveals diverse classes of mRNAs and IncRNAs. Cell 154, 996-1009.

Uchida, N., Hoshino, S., Imataka, H., Sonenberg, N., and Katada, T. (2002). A novel role of the mammalian GSPT/eRF3 associating with poly(A)-binding protein in Cap/Poly(A)-dependent translation. J Biol Chem 277, 50286-50292.

Udem, S.A., and Warner, J.R. (1973). The cytoplasmic maturation of a ribosomal precursor ribonucleic acid in yeast. J Biol Chem 248, 1412-1416.

Valasek, L., Hasek, J., Nielsen, K.H., and Hinnebusch, A.G. (2001). Dual function of elF3j/Hcr1p in processing $20 \mathrm{~S}$ pre-rRNA and translation initiation. J Biol Chem 276, 43351-43360.

Valouev, I.A., Fominov, G.V., Sokolova, E.E., Smirnov, V.N., and Ter-Avanesyan, M.D. (2009). Elongation factor eEF1B modulates functions of the release factors eRF1 and eRF3 and the efficiency of translation termination in yeast. BMC Mol Biol 10, 60.

van den Elzen, A.M., Schuller, A., Green, R., and Seraphin, B. (2014). Dom34-Hbs1 mediated dissociation of inactive $80 \mathrm{~S}$ ribosomes promotes restart of translation after stress. EMBO J 33, 265-276.

Vanrobays, E., Leplus, A., Osheim, Y.N., Beyer, A.L., Wacheul, L., and Lafontaine, D.L. (2008). TOR regulates the subcellular distribution of DIM2, a KH domain protein required for cotranscriptional ribosome assembly and pre-40S ribosome export. RNA 14, 2061-2073.

Venema, J., and Tollervey, D. (1999). Ribosome synthesis in Saccharomyces cerevisiae. Annu Rev Genet 33, 261-311.

von der Haar, T., and Tuite, M.F. (2007). Regulated translational bypass of stop codons in yeast. Trends Microbiol 15, 78-86.

von Moeller, H., Basquin, C., and Conti, E. (2009). The mRNA export protein DBP5 binds RNA and the cytoplasmic nucleoporin NUP214 in a mutually exclusive manner. Nat Struct Mol Biol 16, 247-254.

Weirich, C.S., Erzberger, J.P., Berger, J.M., and Weis, K. (2004). The N-terminal domain of Nup159 forms a beta-propeller that functions in mRNA export by tethering the helicase Dbp5 to the nuclear pore. Mol Cell 16, 749-760.

Weirich, C.S., Erzberger, J.P., Flick, J.S., Berger, J.M., Thorner, J., and Weis, K. (2006). Activation of the DExD/H-box protein Dbp5 by the nuclear-pore protein Gle1 and its coactivator InsP6 is required for mRNA export. Nat Cell Biol 8, 668676.

Wente, S.R., and Rout, M.P. (2010). The nuclear pore complex and nuclear transport. Cold Spring Harb Perspect Biol 2, a000562. 
West, M., Hedges, J.B., Chen, A., and Johnson, A.W. (2005). Defining the order in which Nmd3p and Rpl10p load onto nascent 605 ribosomal subunits. Mol Cell Biol 25, 3802-3813.

White, J., Li, Z., Sardana, R., Bujnicki, J.M., Marcotte, E.M., and Johnson, A.W. (2008). Bud23 methylates G1575 of 18S rRNA and is required for efficient nuclear export of pre-40S subunits. Mol Cell Biol 28, 3151-3161.

Windgassen, M., and Krebber, H. (2003). Identification of Gbp2 as a novel poly(A)+ RNA-binding protein involved in the cytoplasmic delivery of messenger RNAs in yeast. EMBO Rep 4, 278-283.

Windgassen, M., Sturm, D., Cajigas, I.J., Gonzalez, C.I., Seedorf, M., Bastians, H., and Krebber, H. (2004). Yeast shuttling SR proteins Npl3p, Gbp2p, and Hrb1p are part of the translating mRNPs, and Npl3p can function as a translational repressor. Mol Cell Biol 24, 10479-10491.

Winston, F., Dollard, C., and Ricupero-Hovasse, S.L. (1995). Construction of a set of convenient Saccharomyces cerevisiae strains that are isogenic to S288C. Yeast $11,53-55$.

Woolford, J.L., Jr., and Baserga, S.J. (2013). Ribosome biogenesis in the yeast Saccharomyces cerevisiae. Genetics 195, 643-681.

Wu, H., Becker, D., and Krebber, H. (2014). Telomerase RNA TLC1 shuttling to the cytoplasm requires mRNA export factors and is important for telomere maintenance. Cell Rep 8, 1630-1638.

Yao, W., Roser, D., Kohler, A., Bradatsch, B., Bassler, J., and Hurt, E. (2007). Nuclear export of ribosomal $60 \mathrm{~S}$ subunits by the general mRNA export receptor Mex67-Mtr2. Mol Cell 26, 51-62.

Yao, Y., Demoinet, E., Saveanu, C., Lenormand, P., Jacquier, A., and FromontRacine, M. (2010). Ecm1 is a new pre-ribosomal factor involved in pre-60S particle export. RNA 16, 1007-1017.

Yarunin, A., Panse, V.G., Petfalski, E., Dez, C., Tollervey, D., and Hurt, E.C. (2005). Functional link between ribosome formation and biogenesis of iron-sulfur proteins. EMBO J 24, 580-588.

Zakalskiy, A., Hogenauer, G., Ishikawa, T., Wehrschutz-Sigl, E., Wendler, F., Teis, D., Zisser, G., Steven, A.C., and Bergler, H. (2002). Structural and enzymatic properties of the AAA protein Drg1p from Saccharomyces cerevisiae.

Decoupling of intracellular function from ATPase activity and hexamerization. J Biol Chem 277, 26788-26795.

Zemp, I., Wild, T., O'Donohue, M.F., Wandrey, F., Widmann, B., Gleizes, P.E., and Kutay, U. (2009). Distinct cytoplasmic maturation steps of $40 \mathrm{~S}$ ribosomal subunit precursors require hRio2. J Cell Biol 185, 1167-1180.

Zenklusen, D., Vinciguerra, P., Strahm, Y., and Stutz, F. (2001). The yeast hnRNPLike proteins Yra1p and Yra2p participate in mRNA export through interaction with Mex67p. Mol Cell Biol 21, 4219-4232.

Zhao, J., Jin, S.B., Bjorkroth, B., Wieslander, L., and Daneholt, B. (2002). The mRNA export factor Dbp5 is associated with Balbiani ring mRNP from gene to cytoplasm. EMBO J 21, 1177-1187.

Zhouravleva, G., Frolova, L., Le Goff, X., Le Guellec, R., Inge-Vechtomov, S., Kisselev, L., and Philippe, M. (1995). Termination of translation in eukaryotes is governed by two interacting polypeptide chain release factors, eRF1 and eRF3. EMBO J 14, 4065-4072.

Zimmerman, C., Klein, K.C., Kiser, P.K., Singh, A.R., Firestein, B.L., Riba, S.C., and Lingappa, J.R. (2002). Identification of a host protein essential for assembly of immature HIV-1 capsids. Nature 415, 88-92. 


\section{ACKNOWLEDGEMENTS - DANKSAGUNG}

Ich möchte mich ganz herzlich bei Prof. Dr. Heike Krebber dafür bedanken, dass ich in Ihrer Arbeitsgruppe an den interessanten Themen arbeiten durfte sowie für die zahlreichen anregenden Diskussionen, ihre enge Betreuung, ihr Vertrauen und die wissenschaftliche Förderung. Des Weiteren danke ich den Mitgliedern meines thesis committees Prof. Dr. Gerhard Braus und Prof. Dr. Marina Rodnina für die Begleitung meiner Doktorarbeit und ihre wertvollen Anmerkungen.

Auch bedanke ich mich bei der „Göttinger Graduiertenschule für Neurowissenschaften, Biophysik und Molekulare Biowissenschaften“ für die wissenschaftlichen Weiterbildungskurse und die finanzielle Unterstützung von Reisen zu Fachtagungen und nach Prag.

Ein großer Dank geht an Dr. Achim Dickmanns für seine guten Ratschläge zur erfolgreichen Proteinaufreinigung und an die AG Ficner und die AG Braus, die durch das Bereitstellen von Geräten, Stämmen und Plasmiden diese Arbeit unterstützt haben. Weiterhin möchte ich mich sehr bei Dr. Leoš Valášek für die Einladung in sein Labor nach Prag und bei seiner Mitarbeiterin Dr. Lucie Cuchalová für die Unterweisung in der Dichtegradientenzentrifugation bedanken.

Ich bedanke mich herzlich bei Dr. Alexandra Hackmann und Haijia Wu für die gute Zusammenarbeit beim Transportprojekt und Michael Mitter und Lysann Henker für die Aufreinigung von Mex67-Mtr2. Des Weiteren danke ich den von mir betreuten Masterstudenten Irene Hampe, Simon Uhse und Svenja Niehus, die durch ihre Versuche und ihr großartiges Engagement auch zum Gelingen dieser Doktorarbeit beigetragen haben.

Herzlichen Dank an alle aktuellen und ehemaligen Mitglieder der AG Krebber für die tatkräftige Unterstützung bei allen großen und kleinen Problemen, den wunderbaren Zusammenhalt und die unzähligen hilfreichen Diskussionen. Dr. Wilfried Kramer danke ich vor allem für sein kritisches Nachfragen und seine guten Ideen. Ein ganz besonderer Dank geht an Dr. Claudia Baierlein und Lysann Henker, die mich in die Welt der Hefen eingeführt haben, jederzeit ein offenes Ohr hatten und immer für mich da waren - im Labor als auch außerhalb. Ebenso danke ich Lena Oldehaver und Gesa Zander für die sehr angenehme gemeinsame Zeit im Labor und ihren stetigen Beistand.

Für das Korrekturlesen dieser Arbeit danke ich sehr Prof. Heike Krebber, Dr. Heidi Kremling, Lysann Henker, Gesa Zander und Sabrina Beckmann.

Auch möchte ich mich ganz herzlich bei meiner Familie, meinen Freunden und besonders bei Stefan bedanken, dass sie mich jederzeit liebevoll unterstützt, an mich geglaubt und mich motiviert haben. Danke Stefan, dass du mir immer den Rücken freigehalten hast und mir auch in schwierigen Phasen eine Stütze gewesen bist. Ohne euch wäre diese Arbeit nicht möglich gewesen. 